\title{
IntechOpen
}

\section{Advances in \\ Array Optimization}

Edited by Ertugrul Aksoy 



\section{Advances in Array Optimization \\ Edited by Ertugrul Aksoy}



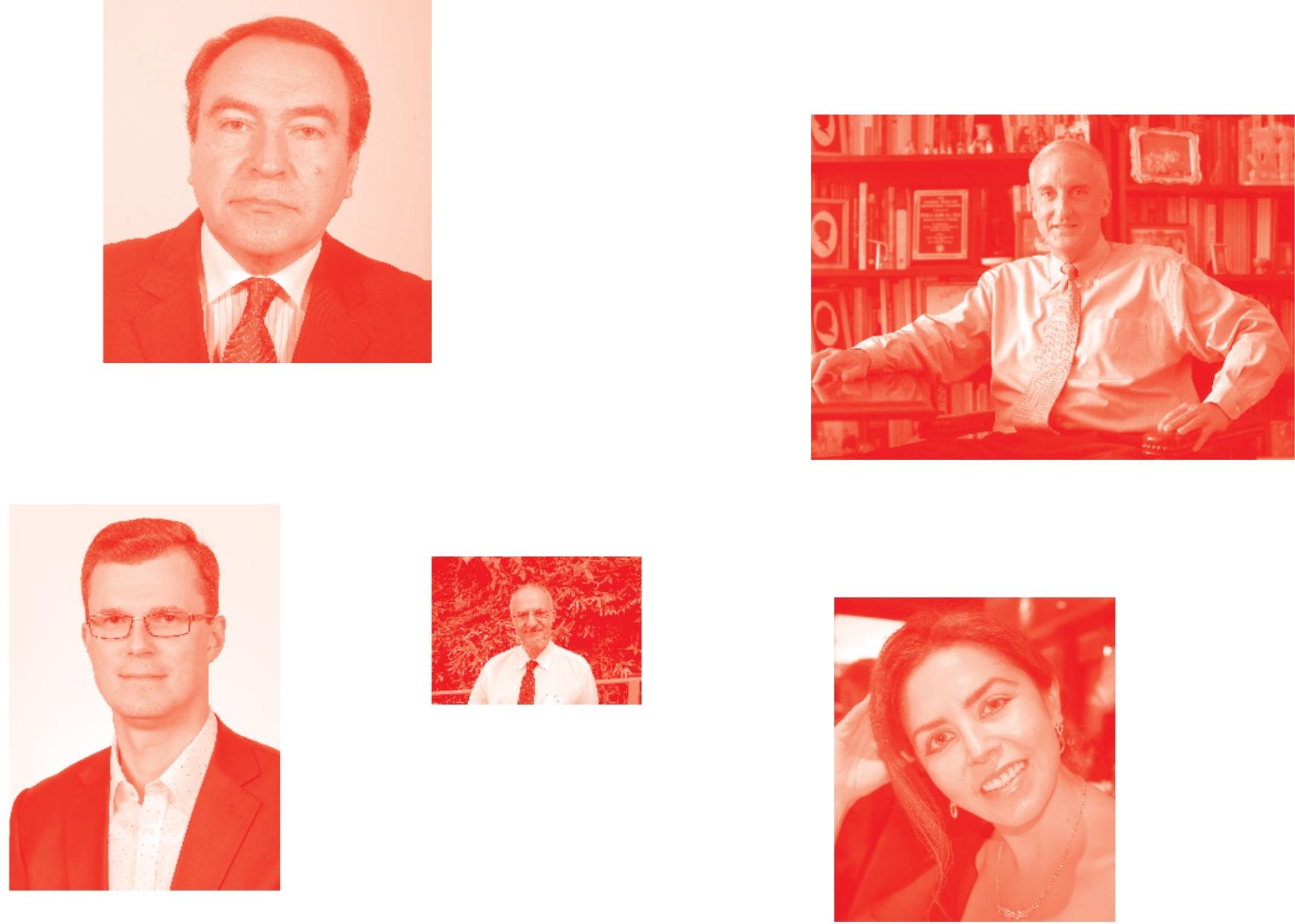

Supporting open minds since 2005
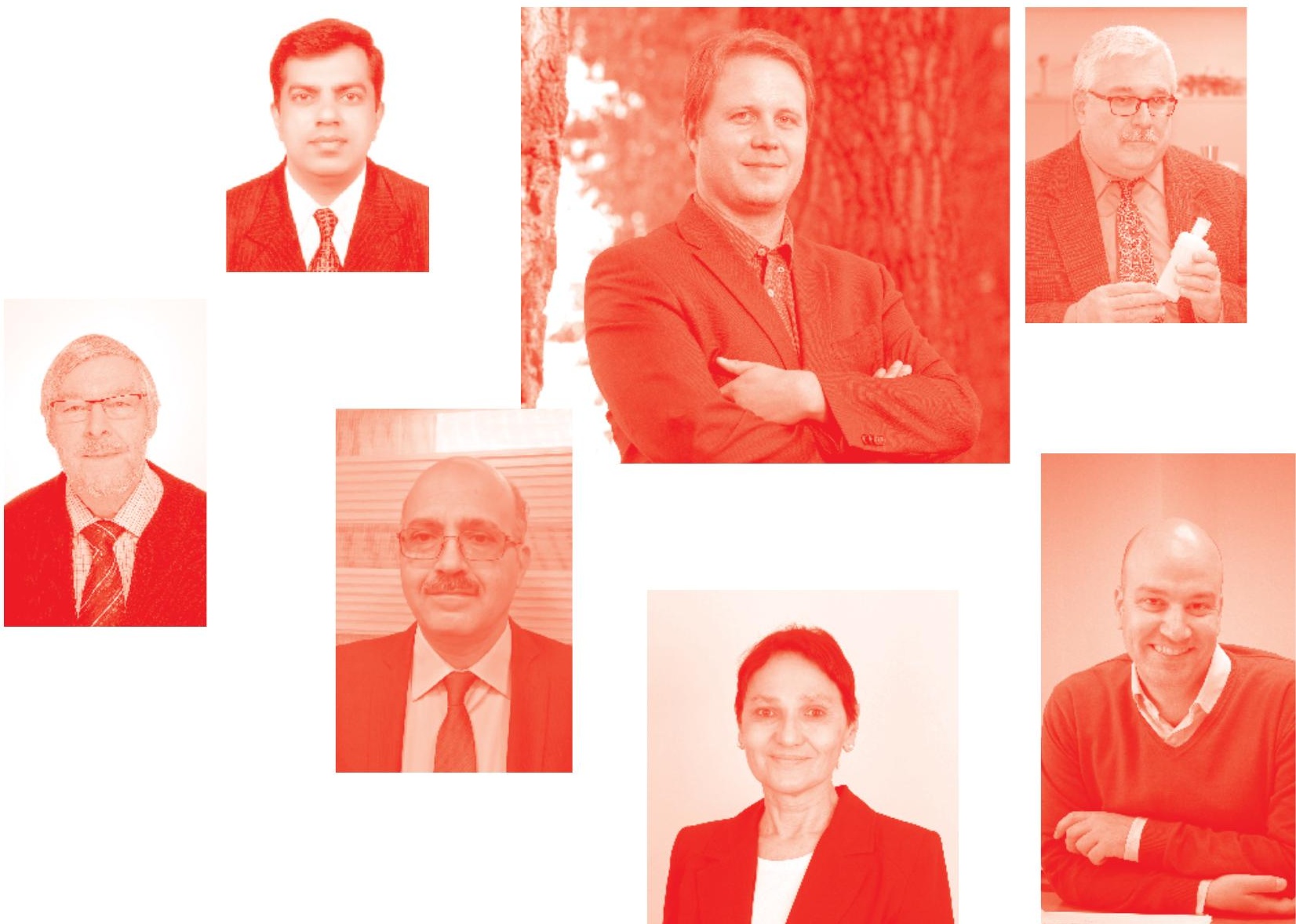
Advances in Array Optimization

http: //dx. doi. org/10.5772/intechopen. 83276

Edited by Ertugrul Aksoy

Contributors

Sujit Kumar Mandal, Ananya Mukherjee, Sujoy Mandal, Tanmoy Das, P. Venu Madhav, M. Siva Ganga Prasad, Hui Chen, Qun Wan, Daniel Rodríguez Prado, Manuel Arrebola, Marcos Rodríguez Pino, Masato Saito, Mikhail E. Belkin, Dmitriy A. Fofanov, Tatiana N. Bakhvalova, Alexander S. Sigov, Irfan Ullah, Benjamin D. Braaten, Shahid Khattak

( ) The Editor(s) and the Author(s) 2020

The rights of the editor(s) and the author(s) have been asserted in accordance with the Copyright, Designs and Patents Act 1988. All rights to the book as a whole are reserved by INTECHOPEN LIMITED. The book as a whole (compilation) cannot be reproduced, distributed or used for commercial or non-commercial purposes without INTECHOPEN LIMITED's written permission. Enquiries concerning the use of the book should be directed to INTECHOPEN LIMITED rights and permissions department (permissions@intechopen.com).

Violations are liable to prosecution under the governing Copyright Law .

\section{(cc) BY}

Individual chapters of this publication are distributed under the terms of the Creative Commons Attribution 3.๑ Unported License which permits commercial use, distribution and reproduction of the individual chapters, provided the original author(s) and source publication are appropriately acknowledged. If so indicated, certain images may not be included under the Creative Commons license. In such cases users will need to obtain permission from the license holder to reproduce the material. More details and guidelines concerning content reuse and adaptation can be found at http : //www . intechopen . com/copyright-policy . html .

\section{Notice}

Statements and opinions expressed in the chapters are these of the individual contributors and not necessarily those of the editors or publisher. No responsibility is accepted for the accuracy of information contained in the published chapters. The publisher assumes no responsibility for any damage or injury to persons or property arising out of the use of any materials, instructions, methods or ideas contained in the book.

First published in London, United Kingdom, 2020 by IntechOpen IntechOpen is the global imprint of INTECHOPEN LIMITED, registered in England and Wales, registration number: 11086078 , 7th floor, 10 Lower Thames Street, London,

EC3R 6AF, United Kingdom

Printed in Croatia

British Library Cataloguing-in-Publication Data

A catalogue record for this book is available from the British Library

Additional hard and PDF copies can be obtained from orders@intechopen.com

Advances in Array Optimization

Edited by Ertugrul Aksoy

p. cm.

Print ISBN 978-1-83880-107-6

Online ISBN 978-1-83880-108-3

eBook (PDF) ISBN 978-1-78923-900-3 


\section{We are IntechOpen, \\ the world's leading publisher of Open Access books}

\section{Built by scientists, for scientists}

\section{$4,600+$}

Open access books available

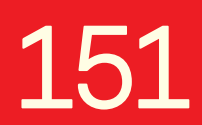

Countries delivered to

\section{$120,000+$}

International authors and editors

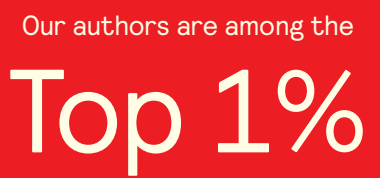

most cited scientists

Contributors from top 500 universities
$135 \mathrm{M}+$

Downloads
1200

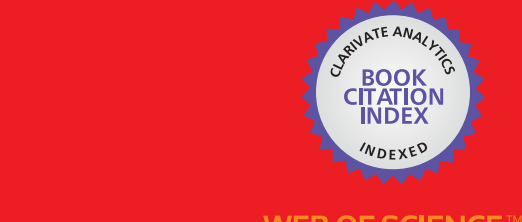

Selection of our books indexed in the Book Citation Index in Web of Science ${ }^{\mathrm{TM}}$ Core Collection (BKCI)

\section{Interested in publishing with us? \\ Contact book.department@intechopen.com}

Numbers displayed above are based on latest data collected.

For more information visit www.intechopen.com 



\section{Meet the editor}

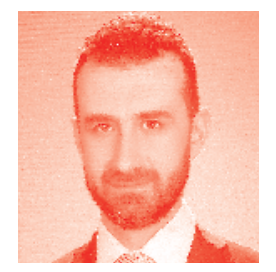

Dr. Ertugrul Aksoy received his MSc and PhD degrees in Electrical and Electronics Engineering from Gazi University, Ankara, Turkey, in 2008 and 2012, respectively. He has been serving as an associate professor in Gazi University since 2017. He has authored or co-authored more than 60 peer-reviewed scientific papers on antenna arrays and optimization fields. He has also served as a reviewer in many journals and as a technical program committee member in many international conferences. His current research interests include antenna engineering, computational electromagnetics, evolutionary optimization, temporal arrays, and meta and frequency selective structures in electromagnetic engineering. Dr. Aksoy is a member of the IEEE Antennas and Propagation Society and IEEE Computational Intelligence Society. 



\section{Contents}

$\begin{array}{lll}\text { Preface } & \text { XIII }\end{array}$

Section 1

Array Optimization

Chapter 1

Pattern Synthesis in Time-Modulated Arrays Using Heuristic

Approach

by Sujit Kumar Mandal, Ananya Mukherjee, Sujoy Mandal and

Tanmoy Das

Chapter 2

Convex Optimization and Array Orientation Diversity-Based

Sparse Array Beampattern Synthesis

by Hui Chen and Qun Wan

Chapter 3

Reflectarray Pattern Optimization for Advanced Wireless

Communications

by Daniel Rodríguez Prado, Manuel Arrebola and Marcos

Rodríguez Pino

Chapter 4

Antenna Pattern Multiplexing for Enhancing Path Diversity

by Masato Saito

Chapter 5

Broadside Pattern Correction Techniques for Conformal Antenna

Arrays

by Irfan Ullah, Shahid Khattak and Benjamin D. Braaten

Section 2

Elements and Feed Network

Chapter 6

Design of Reconfigurable Multiple-Beam Array Feed Network

Based on Millimeter-Wave Photonics Beamformers

by Mikhail E. Belkin, Dmitriy A. Fofanov, Tatiana N. Bakhvalova

and Alexander S. Sigov 
Characterization of Printed Podal Vivaldi Antenna (8-18 GHz) on RT Duroid with Single and Double Cavity by P. Venu Madhav and M. Siva Ganga Prasad 


\section{Preface}

The material in this book provides seven individual research studies on the optimization of array structures from different aspects. Referring to the contents of the book, the studies have been classified in two main parts: "array optimization," which covers the pattern-based approaches in electromagnetic and communications, and "elements and feed network," which covers the element and feed network design. I would like to congratulate all the authors for their valuable contributions and to thank IntechOpen, who made this book project possible. I hope this book will contribute to the scientific community dealing with the optimization of array structures.

Ertugrul Aksoy, PhD

Department of Electrical and Electronics Engineering, Faculty of Engineering, Gazi University, Turkey 

Section 1

Array Optimization 



\title{
Pattern Synthesis in Time-Modulated Arrays Using Heuristic Approach
}

\author{
Sujit Kumar Mandal, Ananya Mukherjee, Sujoy Mandal \\ and Tanmoy Das
}

\begin{abstract}
Time-modulation principle evolves as an emerging technology for easy realization of the desired array patterns with the help of an additional degree of freedom, namely, "time." To the antenna community, the topic, time-modulated antenna array (TMAA) or 4D antenna arrays, has got much attention during the last two decades. However, population-based, stochastic, heuristic evolutionary algorithm plays as an important protagonist to meet the essential requirements on synthesizing the desired array patterns. This chapter is basically devoted to understand the theory of different time-modulation principles and the application of optimization techniques in solving different antenna array synthesis problems. As a first step, the theory of time-modulation principles and the behaviors of the sideband radiation (SBR) that appeared due to time modulation have been studied. Then, different important aspects associated with TMAA synthesis problems have been discussed. These include conflicting parameters, the need of evolutionary algorithms, multiple objectives and their optimization, cost function formation, and selection of weighting factors. After that, a novel approach to design a time modulator for synthesizing TMAAs is presented. Finally, discussing the working principle of an efficient heuristic approach, namely, artificial bee colony $(A B C)$ algorithm, the effectiveness of the time modulator and potentiality of the algorithm are presented through representative numerical examples.
\end{abstract}

Keywords: antenna array synthesis, side lobe level (SLL), time modulation, sideband radiation, sideband level (SBL), evolutionary algorithms

\section{Introduction}

In any wireless communication system, the antenna is an essential component to transmit or receive a message signal. In many applications such as satellite communication, point-to-point communication, military communication, surveillance, radar, sonar, aircraft, etc., the antenna gain and directivity should be sufficiently high so as to direct most of the antenna-radiated power along a particular direction by reducing the power level (side lobe power) at other directions. A single radiator may not meet such requirements due to its omnidirectional power pattern and high side lobe level (SLL) in the far-field region. Moreover, radiation of huge amount of 
transmitter power from a single antenna element needs high-power amplification in the feed network. The high-power amplifier is not easy to design and safe to handle. Therefore, a number of antenna elements are arranged along a line, called linear antenna array (LAA), or in a plane called planer antenna array (PAA). The use of multiple antenna elements in the transmission and reception systems simplifies the power amplifier design problem by reducing the power level per transmitting antenna elements of the arrays. Some other advantages of using antenna arrays are to improve signal fading resistance or deliberately exploit the signal fading; mitigate the interfering signal coming from other directions, adaptive beam forming, and null steering at both transmitter and receiver; and increase system capacity. Due to its high gain and narrow beamwidth, the large antenna arrays also find applications in weather forecast, astronomy, image processing, and biomedical imaging.

Although the antenna array with uniform excitation amplitude and equally spaced antenna elements is the simplest one for practical implementation and also can be used to synthesize different patterns, due to the high value of peak SLL, it is impractical to use in such applications. In conventional antenna array (CAA) system, the low side lobe pattern is obtained by tapering the static excitation amplitudes. The well-known analytical techniques to taper amplitude distributions in nonuniformly excited antenna arrays are Dolph-Chebyshev (DC) and Taylor series [1]. However, the high dynamic range ratio (DRR) and complex excitation of the antenna elements are the major drawbacks of such CAA synthesis method with nonuniform excitation, because the complex excitation is practically difficult to realize and designing the practical antenna with high DRR of static amplitude tapering provides various errors such as systematic errors and random errors.

Conversely, the ultralow SLL pattern in the far-field of the antenna array can be realized even in uniform amplitude antenna arrays by exploiting "time" as a fourth dimension $[2,3]$. The introduction of the additional dimension "time," into the antenna array system, results in time-modulated antenna array (TMAA). By using the fourth degree of freedom, "time" in antenna array system, various errors in realizing the low SLL pattern can be drastically reduced, and error tolerance levels become equivalent to those obtained in conventional antenna array system for the patterns of ordinary SLLs $[4,5]$. Yet, the main disadvantage in TMAA is the generation of sideband signals which appeared due to the time modulation of the antenna signals by periodically commutating the antenna elements with the specified modulation frequency. Therefore, time modulation involves with the radiation or reception of electromagnetic energy at different harmonics of the modulation frequency that are termed as sidebands. In some applications where the antenna array is synthesized at center (operating) frequency, sideband signals are not useful. In such cases, sideband signals and associated power losses are suppressed to improve the radiation efficiency at the operating frequency of the antenna array $[5,6]$. Presently, it is investigated that sideband signals are also effective in synthesizing multiple patterns and researchers are interested to exploit the same in some specific applications of the modern-day communication systems like harmonic beam forming [7], generation of multibeam radiation pattern [8], beam steering [9, 10], direction finding [11], wireless power transmission [12], etc. The interested readers may refer to Reference [13] for the stateof-the-art overview, applications, and present research trend on time-modulation theory and techniques.

This chapter explains about the fundamental theory and techniques of different time-modulation strategies and such antenna array synthesis methods using optimization algorithms. The parameters involved with the use of optimization techniques and TMAA synthesis problem have also been presented. 


\section{Theory of time-modulated antenna array (TMAA)}

Let us consider a linear antenna array of $\mathrm{N}$ number of mutually uncoupled isotropic radiators with inter-element spacing $d_{0}$. The antenna elements are placed along the $\mathrm{x}$-axis with the first element at the origin of the geometrical coordinate system as shown in Figure 1. In the XZ plane (one of the vertical principle plane), the array factor expression of CAAs can be obtained as in Eq. (1) [1]:

$$
A F^{c}=\sum_{p=1}^{N} A_{p} e^{j \Phi_{p}} e^{j\left[\omega_{0} t+(p-1) \beta d_{0} \cos \theta\right]}
$$

where $\omega_{0}=2 \pi f_{0}=2 \pi / T_{0}$ is the angular frequency in $\mathrm{rad} / \mathrm{sec}$ for the operating signal of frequency $f_{0}$ in $\mathrm{Hz} ; T_{0}$ is the time period of the operating signal; $\beta=2 \pi / \lambda$ is the wave number with $\lambda$ being the wavelength; $p=1, \ldots \ldots, N$ represents the element number of the antenna array; $A_{p}$ and $\Phi_{p} \forall p \in[1, N]$ stand for the normalized static excitation amplitudes and phases of the array elements, respectively; and $\theta$ is the angle made by the line joining the observing point and the origin with the $\mathrm{x}$-axis as shown in Figure 1.

In order to control the antenna pattern by using the additional degree of freedom, namely, "time," periodically the static excitation amplitudes of the antenna element are time-modulated. The commonly used and simplest way of doing that is to insert high-speed radio-frequency $(\mathrm{RF})$ switches in the feed network, just prior to radiating sources as shown in Figure 2. Each array element is assumed to be connected to the RF switches with individually controlled switching circuits. The switches are periodically "on" and "off" according to a predetermined on-time sequence $t_{p}^{o n}\left(0 \leq t_{p}^{o n} \leq T_{m}\right) \forall p \in[1, N]$, with time period, $T_{m}$. The switching rate, $f_{m}=1 / T_{m}$, is selected such that if the maximum frequency of the message signal is $f_{\text {max }}(\mathrm{Hz}), T_{0}<<T_{m} \leq \frac{1}{f_{\max }}$ [14]. Thus, during each period, the on-time duration by which a switch is on, the array element connected to that switch is active for that time duration only; otherwise, it will be inactive.

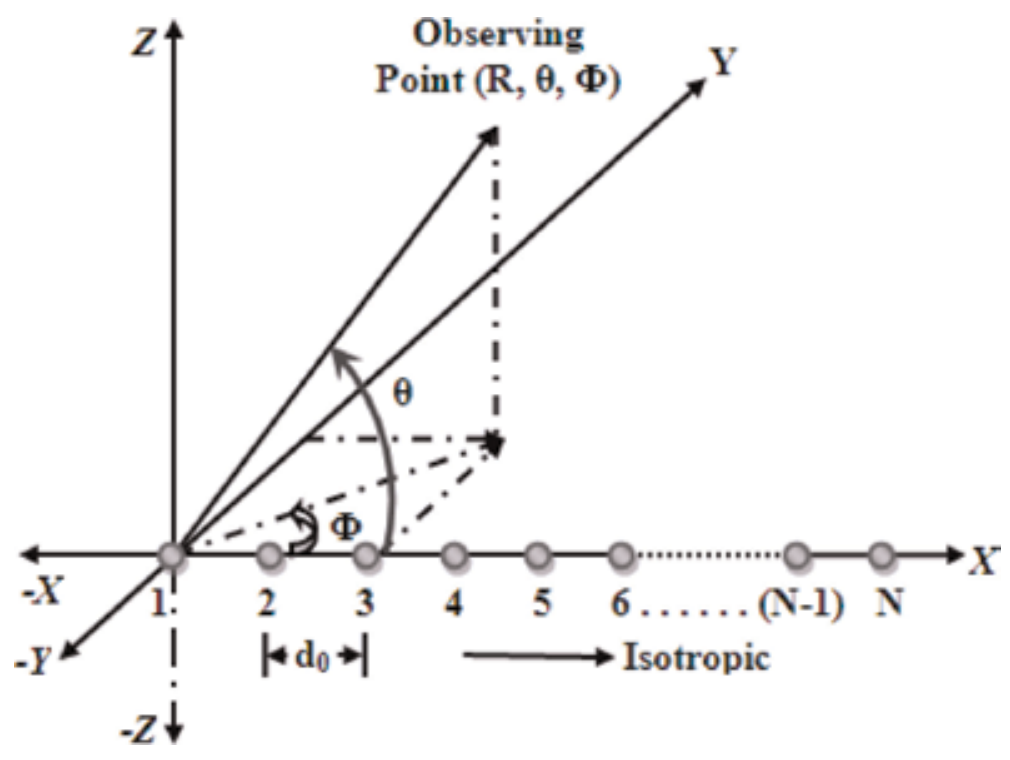

Figure 1.

Basic antenna array of $N$ element with inter-element spacing of $d_{o}$. 
Let us further assume that all the switches corresponding to the antenna elements in Figure 2 are on (short circuited) at the same instant of time, say at the beginning of each period " $\eta{ }^{*} T_{m}$ " with " $\eta$ " being the time period number $0,1,2, \ldots$, by using rectangular pulses of amplitude unity. Hence, the switches which are on for the whole time period $T_{m}$ as shown in Figure 3(a) can be directly connected to the signal as time modulation is not required for such cases. On the other hand, the switches remained short circuited for their specific on-time duration and open

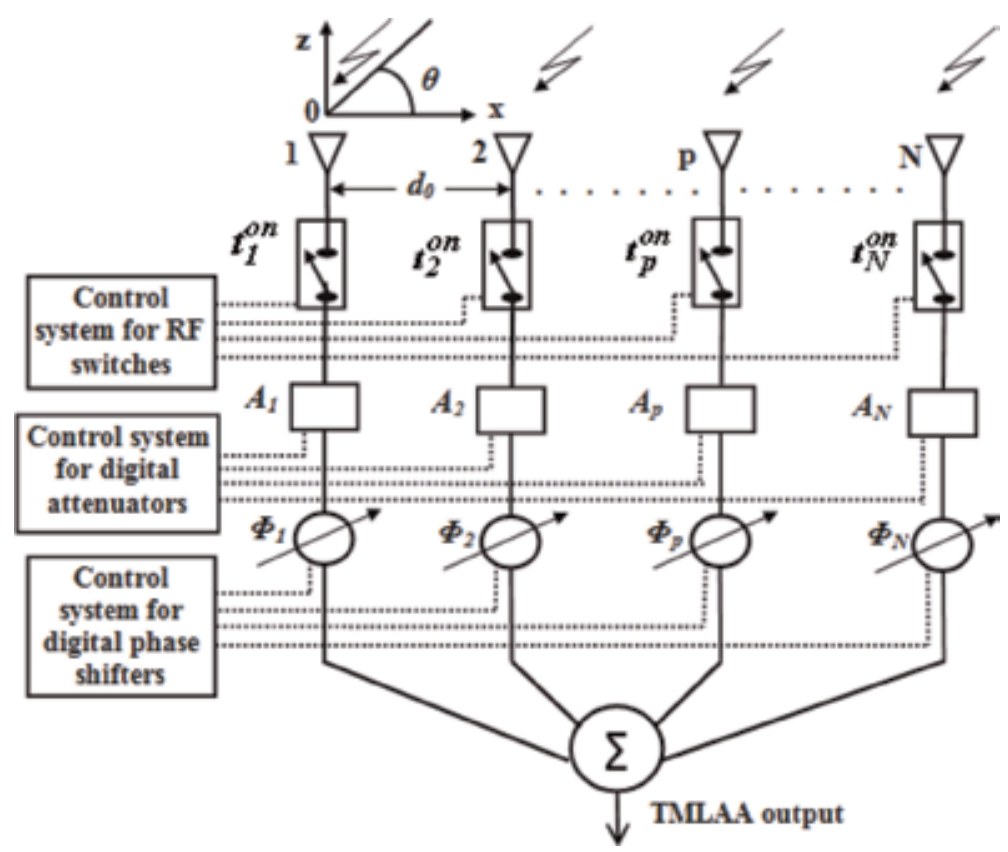

Figure 2.

Time-modulated linear antenna array (TMLAA) geometry.

(a)



(b)

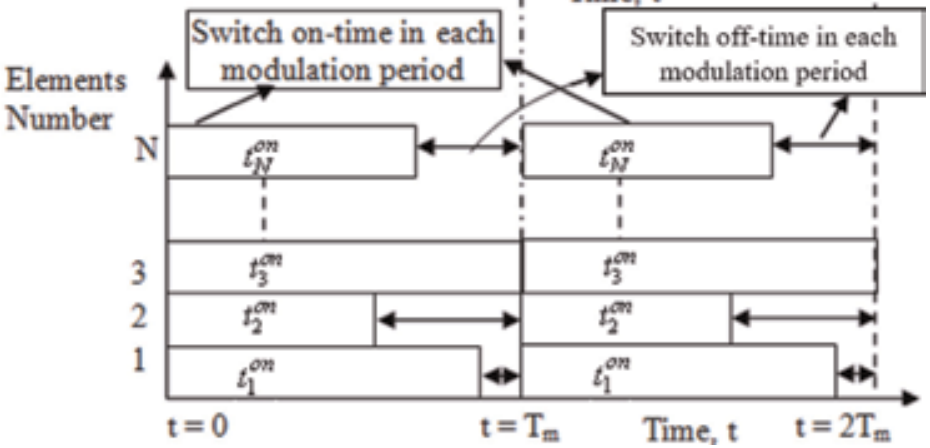

Figure 3 .

The periodic pulse sequence of the TMLAA. (a) Unit pulse of periodicity $T_{P} .(b)$ On-off time duration of each antenna elements for one time-modulation period $T_{P}$, and it is repeated at every $T_{P}$ time interval. 
circuited after their corresponding on-time duration $\left(t_{p}^{o n}\right)$ as shown in Figure 3(b). Figure 3(b) shows the on-off time sequence of the switches for the first two time periods only. The same process is repeated in the next consecutive periods. Thus the switching function of the $p^{t h}$ element can be expressed by a periodic pulse $U_{p}(t)$, such that at each period

$$
U_{p}(t)=\left\{\begin{array}{cc}
1 ; & \eta T_{m} \leq t \leq\left(\eta T_{m}+t_{p}^{o n}\right) \\
0 ; & \text { elsewhere }
\end{array}\right.
$$

After the switching operation, the array factor expression of Eq. (1) can be written as in Eq. (3) [2]:

$$
A F(\theta, t)=\sum_{p=1}^{N} U_{p}(t) A_{p} e^{j\left[\omega_{0} t+\alpha_{p}+\Phi_{p}\right]}
$$

where $\alpha_{p}=(p-1) \beta d_{0}\left\{\cos \theta-\cos \theta_{0}\right\}$ is the linear progressive phase shift of $p^{\text {th }}$ element and $\theta_{0}$ is the direction of maximum radiation. As $U_{p}(t)$ in Eq. (3) is a time periodic function of periodicity $T_{m}$, it can be decomposed by applying Fourier series technique as

$$
U_{p}(t)=\sum_{k=-\infty}^{k=+\infty} C_{p k} e^{j k \omega_{m} t}
$$

where $\omega_{m}=2 \pi / T_{m}=2 \pi f_{m}$ is the modulation frequency and $C_{p k}$ is the Fourier coefficient at the $k^{\text {th }}$ harmonics for the $p^{\text {th }}$ element and is obtained as $[5,14]$

$$
C_{p k}=\tau_{p} \frac{\sin \left(k \pi \tau_{p}\right)}{k \pi \tau_{p}} e^{-j k \pi \tau_{p}}
$$

where $\tau_{p}=t_{p}^{o n} / T_{m} \forall p \in[1, N]$ stand for the normalized on-time durations of the array elements.

Putting Eq. (4) in Eq. (3), the array factor expression of Eq. (3) is obtained as

$$
A F(\theta, t)=\sum_{k=-\infty}^{k=+\infty} \sum_{p=1}^{N} A_{p} C_{p k} e^{j\left(\Phi_{p}+\alpha_{p}\right)} e^{j\left(\omega_{0}+k \omega_{m}\right) t}
$$

Thus, Eq. (6) expresses that the signal is not only radiated at the operating frequency, $\omega_{0}$ for $k=0$, but also the signals are radiated at different harmonics of the modulating frequency, $k \omega_{m}$, with $\omega_{0}$ as the center frequency. The signal radiation at different harmonics is termed as sideband radiation (SBR). For such a TMLAA, the array factor expression at $k$ th harmonic of the modulation frequency is readily obtained by combining Eqs. (5) and (6) as

$$
A F_{k}(\theta, t)=e^{j\left(\omega_{0}+k \omega_{m}\right) t} \sum_{p=1}^{N} A_{p} \tau_{p} \frac{\sin \left(k \pi \tau_{p}\right)}{k \pi \tau_{p}} e^{-j\left[k \pi \tau_{p}-\left(\Phi_{p}+\alpha_{p}\right)\right]}
$$

Therefore, the array factor at the fundamental frequency, i.e., at operating frequency (for $k=0$ ) and at the first two positive harmonics (for $k=1$ and $k=2$ ), is obtained as in Eqs. (8), (9), and (10), respectively: 


$$
\begin{gathered}
A F_{0}(\theta, t)=e^{j \omega_{0} t} \sum_{p=1}^{N} A_{p} \tau_{p} e^{j\left(\Phi_{p}+\alpha_{p}\right)} \\
A F_{1}(\theta, t)=\frac{e^{j\left(\omega_{0}+\omega_{m}\right) t}}{\pi} \sum_{p=1}^{N} A_{p} \sin \left(\pi \tau_{p}\right) e^{-j\left[\pi \tau_{p}-\left(\Phi_{p}+\alpha_{p}\right)\right]} \\
A F_{2}(\theta, t)=\frac{e^{j\left(\omega_{0}+2 \omega_{m}\right) t}}{2 \pi} \sum_{p=1}^{N} A_{p} \sin \left(2 \pi \tau_{p}\right) e^{-j\left[2 \pi \tau_{p}-\left(\Phi_{p}+\alpha_{p}\right)\right]}
\end{gathered}
$$

From Eq. (8), it can be observed that $\tau_{p}{ }^{\prime} \mathrm{s} \forall p \in[1, N]$ provides an additional flexibility in synthesizing antenna array patterns. For example, making values of $\tau_{p}$ 's $\forall p \in[1, N]$ equivalent to that of the required static excitation to synthesize Dolph-Chebyshev or Taylor series pattern, low SLL patterns can be realized even with uniformly excited array with unit static excitation $A_{p}=1 \forall p \in[1, N]$. Also, Eqs. (9) and (10) indicate that the harmonics radiated from different timemodulated elements are added together at frequencies in multiples of the modulation frequency, $f_{m}$, to produce resultant sideband signals.

\section{Behaviors of sideband radiation (SBR)}

It can be observed from Eqs. (7)-(10) that, due to time modulation, the sideband signals inherently appeared around the center frequency spaced in multiples of the modulation frequency. In this section, the characteristics of harmonic signal radiated by an arbitrary time-modulated element are observed by varying the normalized switch-on time for its complete range from 0 to 1 . Then by defining relative and normalized sideband power, the effects of reducing SLL on the first null beamwidth (FNBW) and maximum sideband power level are observed.

\subsection{Characteristics of harmonic radiations (HRs)}

From Eq. (7), we can see that the array factor at different sidebands is the superposition of the harmonic signal radiated from the individual antenna element. Hence, sideband power pattern and total sideband power can be obtained from the harmonic characteristics of the time-modulated elements as expressed in Eq. (5). The normalized harmonic radiation of the individual time-modulated antenna element is given as [15]

$$
h_{p k}=20 \log _{10}\left|C_{p k}\right| /\left|C_{p 0}\right|=\frac{\sin \left(k \pi \tau_{p}\right)}{k \pi \tau_{p}}
$$

where $h_{p k}$ is the normalized/relative harmonic radiation corresponding to the $p^{\text {th }}$ element. The variation of normalized harmonic power of the first three harmonics ( $k=1,2$, and 3 ) with normalized switch-on time, $\tau_{p}$, over its complete range $(0,1)$ is shown in Figure 4. As can be seen, at the lower value of $\tau_{p}$, all $\mathrm{h}_{\mathrm{pkmax}}$ are almost the same, and for $\tau_{p} \rightarrow 0$, all $\mathrm{h}_{\mathrm{pk} \max }$ are exactly equal to 0 (zero) $\mathrm{dB}$ as it is expected from the Fourier series of unit impulse function. However, at the other extremes of $\tau_{p}$, when $\tau_{p} \rightarrow 1$, all $\mathrm{h}_{\mathrm{pk} \max } \rightarrow-\infty$, which is the predicted result as can be seen in Eqs. (5) to (10), with $k=1,2$, and 3. Again there is no radiation at $\mathrm{h}_{\mathrm{p} 2}$ for $\tau_{p}=0.5$ and at $\mathrm{h}_{\mathrm{p} 3}$ for $\tau_{p}=0.3$ and 0.66 which can also be verified from Eq. (5) with $k=1,2$, and 3. Thus, Figure 4 indicates that the contribution of the harmonic component 


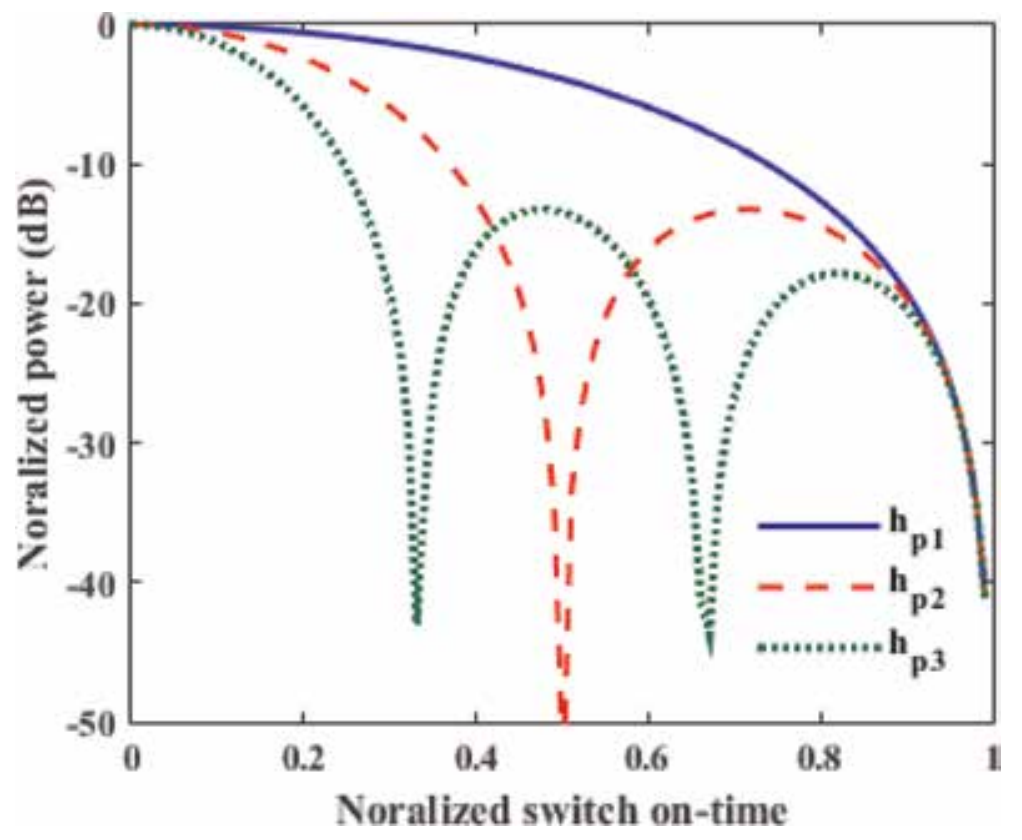

Figure 4 .

Variation of the first three harmonic powers from an antenna element with normalized switch-on time, $\tau_{p}$.

from a particular element to produce the sideband pattern depends on the on-time duration of the corresponding element. Therefore, the desired sideband power pattern can be synthesized in TMAAs by judiciously controlling the on-time sequence of the time-modulated antenna elements.

\subsection{Normalized and relative power}

Usually in TMAA, the radiation pattern is synthesized at center frequency by suppressing the sideband radiation level to sufficiently low value. Thus, the maximum of the power radiated at $f_{0}$ is used to normalize the corresponding power pattern at center frequency. On the other hand, the sideband power is divided by the maximum power at $f_{0}$ to measure the relative power level at different sidebands with respect to that of the radiation at center frequency. In this regard, the relative signal power radiated at different harmonics $(k \neq 0)$ is measured as in Eq. (12):

$$
S B L_{k}(\mathrm{~dB})=20 * \log _{10}\left(A F_{k}(\theta, t) / \max \left(A F_{0}(\theta, t)\right)\right)
$$

where " $S B L_{k}$ " represents the relative value of sideband level at $k^{\text {th }}$ harmonic $(k=1,2, \ldots)$, i.e., relative value of the array factor $A F_{k}$ in $\mathrm{dB}$, and " $\max \left(A F_{0}(\theta, t)\right)$ " is the maximum value of the array factor at operating frequency $\omega_{0}$, i.e., the maximum radiation level at $k=0$. Thus, with $k=0$, Eq. (11) gives the normalized power pattern for the center frequency pattern, whereas, for the sideband radiations (with $k \neq 0$ ), it is the relative power with respect to the maximum of the center frequency pattern.

\subsection{Influence on the sideband level and first null beamwidth during reduction of side lobe level of the fundamental pattern}

It is understood that in addition to the desired operating frequency (center frequency), TMAAs also radiate signals at the infinite number of different 
harmonics of the modulation frequency. When the desired power pattern is synthesized at the center frequency, the sideband power is wasted. In this section, the influences on the first null beamwidth (FNBW) and sideband radiation by reducing SLL of the center frequency pattern are observed. The SLL of the power pattern at $f_{0}$ is reduced by using the conventional amplitude tapering technique, namely, Dolph-Chebyshev (DC) [1], and a heuristic search global optimization method, namely, genetic algorithm (GA) [16].

\subsubsection{SLL reduction using Dolph-Chebyshev technique}

The conventional antenna array synthesis technique such as Dolph-Chebyshev (DC) method [1] can be directly used to realize power pattern of the desired value of SLL at the center frequency. For a 30-element uniformly excited (UE) TMAA, the equivalent excitation coefficient of the DC pattern of desired SLL is made equal to the normalized on-time duration of the array elements. Following the DC method, the power pattern of different values of SLL is obtained at the center frequency.

\subsubsection{SLL reduction using heuristic approach}

In order to reduce the SLL at the center frequency pattern using optimization technique, a cost function is required. A well-defined cost function of any optimization problem is important to obtain satisfactory performance. The cost function measures the distances between the desired and obtained values of the radiation parameters which are to be controlled. During the optimization process, the algorithms compare the obtained values of the radiation parameters with those of their respective desired values. Without considering sideband radiation and FNBW, the cost function to realize the patterns of desired SLLs at $f_{0}$ is defined as

$$
\Psi=\left(S L L_{d}-S L L_{\max }\right)^{2}
$$

where $S L L_{\max }$ is the actual value of the $S L L$ as obtained during each trial of the optimization process and $S L L_{d}$ is its desired value. Any heuristic search global optimization method can be employed to reduce the SLL of the power pattern at $f_{0}$. Here, one of the useful stochastic search global optimization methods, namely, genetic algorithm (GA), is used to synthesize the power pattern of different values of $S L L$ of the array under consideration [17].

\subsubsection{Results and discussion}

It can be seen from Eqs. (5)-(10) that the Fourier coefficients and hence amplitudes of the harmonic signals are decreasing gradually with increasing harmonic order. Thus, the radiation energy at the first few harmonics (called sidebands) is most significant. So, the influence on the maximum radiation at the first two harmonics of TMAA is observed by reducing the $S L L$ of the center frequency pattern. Firstly, the $S L L$ of the power pattern at $f_{0}$ is reduced by using the DolphChebyshev (DC) method [1]. Then a global optimization method is used to synthesize the same pattern as obtained via DC. In order to observe the effects of reducing SLL on SBL and FNBW, these values are noted for different power patterns. Table 1 shows the simulation results of the maximum sideband level $\left(S B L_{\max }\right)$ at the first and second harmonics for the fundamental pattern with different values of maximum $S L L\left(S L L_{\max }\right)$ ranging from $-15 \mathrm{~dB}$ to $-55 \mathrm{~dB}$. The radiation pattern at $f_{0}$ as obtained by GA and DC with $S L L$ of $-55 \mathrm{~dB}$ is shown in Figure 5. The first null 
Pattern Synthesis in Time-Modulated Arrays Using Heuristic Approach DOI: $h t t p: / / d x$. doi.org/10.5772/intechopen.89479

\begin{tabular}{|c|c|c|c|c|c|c|c|}
\hline \multicolumn{4}{|c|}{ The patterns at $f_{o}$ by DC } & \multicolumn{4}{|c|}{ The patterns at $f_{o}$ by GA } \\
\hline $\begin{array}{l}S L L_{\max } \\
\text { (dB) }\end{array}$ & $\begin{array}{c}F N B W \\
\text { (deg) }\end{array}$ & $\begin{array}{c}S B L_{1(\max )} \\
\quad(\mathrm{dB})\end{array}$ & $\begin{array}{c}S B L_{2(\max )} \\
\mathrm{dB})\end{array}$ & $\begin{array}{c}S L L_{\max } \\
\quad(\mathrm{dB})\end{array}$ & $\begin{array}{c}F N B W \\
\text { (deg) }\end{array}$ & $\begin{array}{c}S B L_{1(\max )} \\
\quad(\mathrm{dB})\end{array}$ & $\begin{array}{c}S B L_{2(\max )} \\
\text { (dB) }\end{array}$ \\
\hline-15 & 7.2 & -3.35 & -8.30 & -15.06 & 8.4 & -10.01 & -17.91 \\
\hline-20 & 8.4 & -7.2 & -17.83 & -20.03 & 9.6 & -9.95 & -17.06 \\
\hline-25 & 9.8 & -13.36 & -20.25 & -25.78 & 10.4 & -8.51 & -14.27 \\
\hline-30 & 11.2 & -12.28 & -19.31 & -30.28 & 12.0 & -9.98 & -14.75 \\
\hline-35 & 12.4 & -12.42 & -17.45 & -35.66 & 17.2 & -12.19 & -17.39 \\
\hline-40 & 13.8 & -12.42 & -17.45 & -40.04 & 19.0 & -6.058 & -13.13 \\
\hline-45 & 15.2 & -12.59 & -17.40 & -43.76 & 20.2 & -10.12 & -14.572 \\
\hline-50 & 16.6 & -12.58 & -17.40 & -50.52 & 22.4 & -7.301 & -12.7870 \\
\hline-55 & 18.0 & -12.55 & -17.37 & 55.6 & 23.6 & -7.3 & -12.9 \\
\hline
\end{tabular}

Table 1.

Radiations maximum at the first two sidebands and FNBW for the fundamental patterns of different values of SLL.

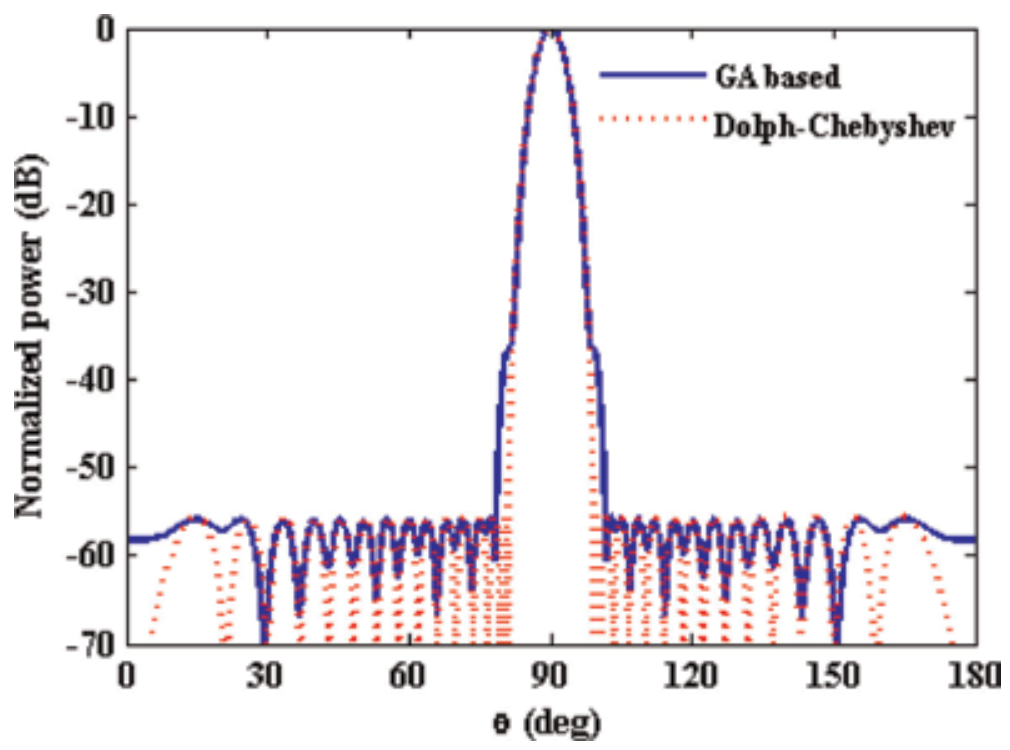

Figure 5.

GA- and Dolph-Chebyshev-based pattern of SLL of $-55.6 \mathrm{~dB}$ at $\mathrm{f}_{\circ}$.

beamwidth (FNBW) for different values of $S L L_{\max }$ of the main beam radiation pattern has been noted and is plotted in Figure 6. The maximum two harmonics are normalized with respect to the maximum value of the radiation at $f_{0}$. For the different values of $S L L$ s, the change in $S B L_{\max }$ at the first and second harmonics is shown Figure 7. Since, the Dolph-Chebyshev (DC) method gives the optimum pattern, i.e., the pattern with minimum $F N B W$ for a specific value of $S L L$ or vice versa. For the DC patterns of different $S L L$ s, the corresponding $F N B W, S B L_{1(\max )}$, and $S B L_{2(\max )}$ are also given in Table 1. The plot SLL vs. FNBW is shown in Figure 6, and that for $S L L$ vs. $S B L_{\max }$ is shown in Figure 7. Figure 6 depicts that for the DC method, FNBW is linearly increased when $\left|S L L_{\max }\right|$ is enhanced, whereas Figure 7 shows that $S B L_{1 \text { max }}$ initially decreases from $-3.35 \mathrm{~dB}$ and obtained its minimum value of $-13.36 \mathrm{~dB}$ at $-25 \mathrm{~dB} S L L$ pattern. Thereafter, it gradually 


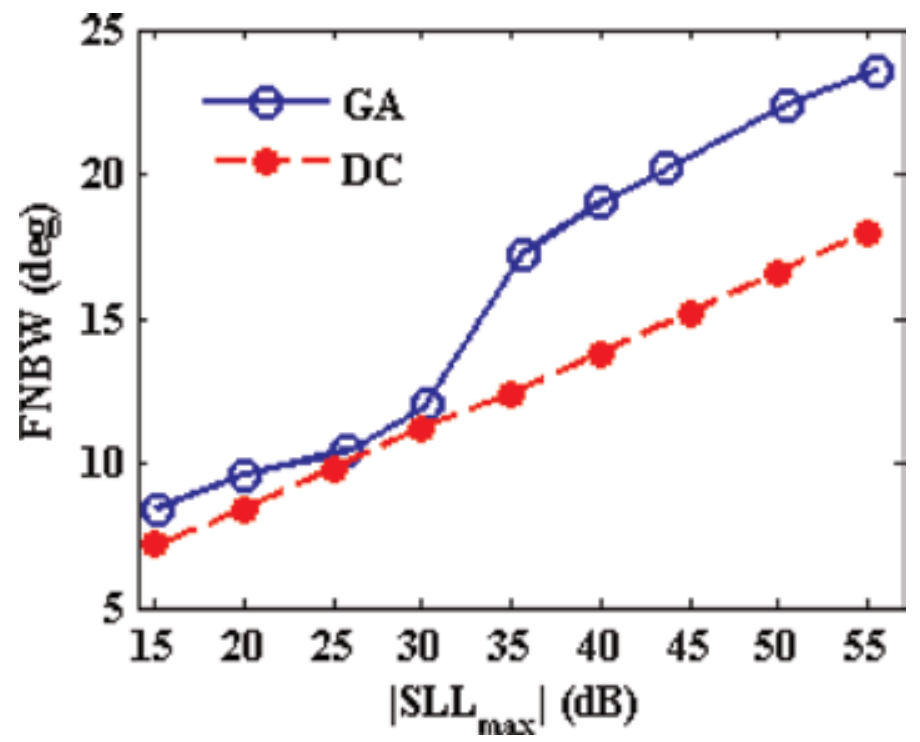

Figure 6.

FNBW for different values of SLLs of the GA and Dolph-Chebyshev patterns at $f_{o}$.

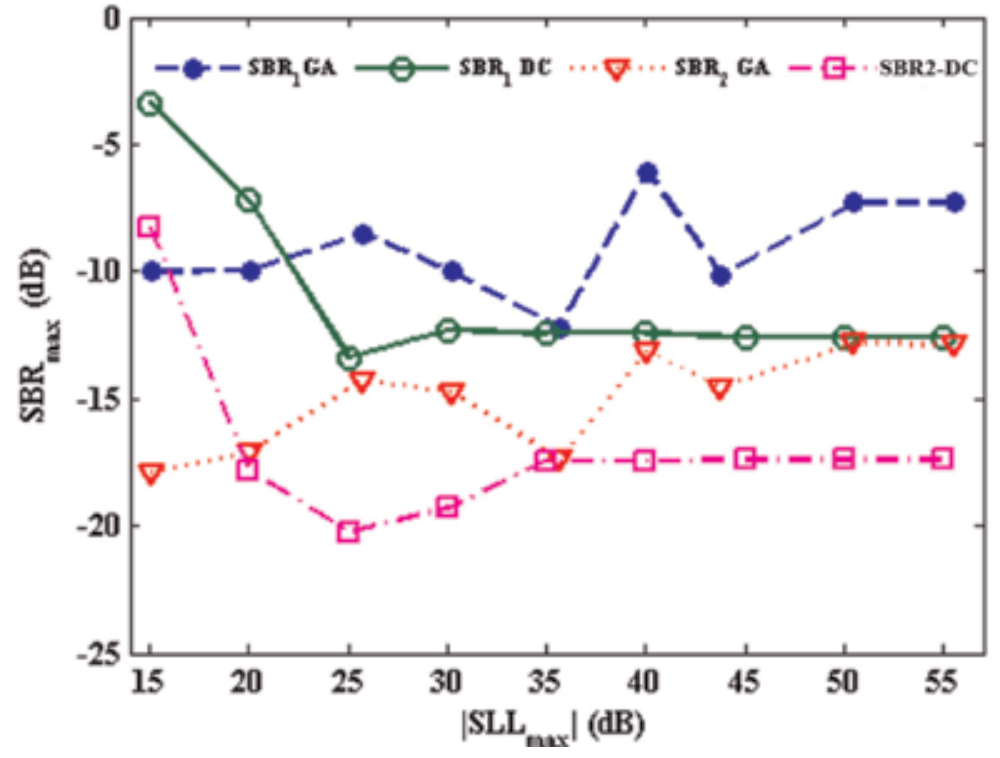

Figure 7.

The plot of $S B R_{1(\max )}$ and $S B R_{2(\max )}$ for the different values of $S L L s$ of the patterns at $f_{o}$.

increases and becomes almost steady at $-12.5 \mathrm{~dB}$ after $-30 \mathrm{~dB}$ SLL. From Figures 6 and 7, it can be seen that for the GA-based patterns of different SLLs, $S B L_{\max }$ and $F N B W$ vary randomly as in the cost function, only $S L L$ is considered without controlling FNBW and SBL.

\section{Time-modulation strategies}

Different time-modulation strategies have been reported for synthesizing antenna arrays. These can be classified as (1) variable aperture size (VAS); (2) pulse 


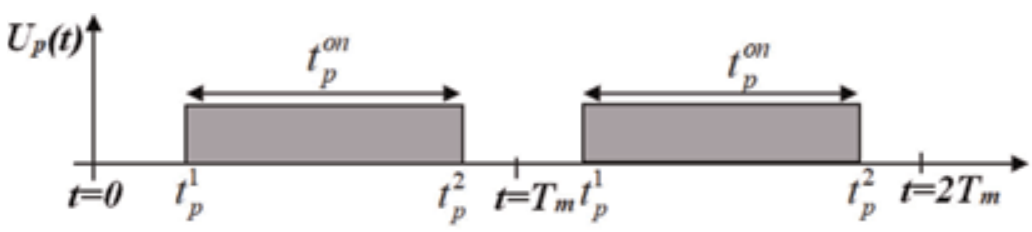

(a)

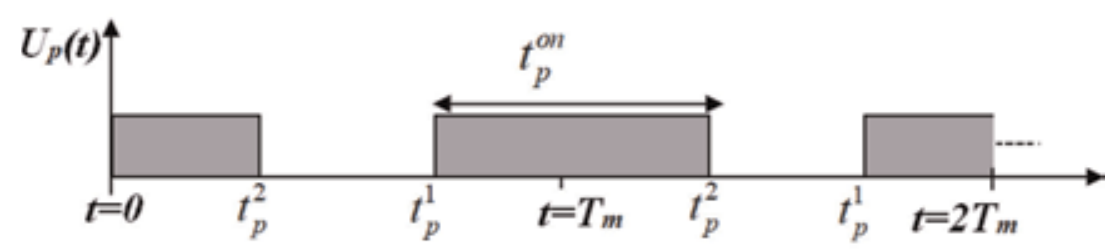

(b)

Figure 8.

Switching instants defining pulse shifting strategy under two cases.

shifting; (3) binary optimized time sequence (BOTS); (4) subsectional optimized time steps (SOTS); (5) variable aperture size (VAS) with quantized on-time (VAS-QOT) or quantized aperture size (QAS); and (6) nonuniform period modulation (NPM). From the array factor expression as given in Eq. (6), it can be observed that for TMAA, the array factor at different harmonic can be obtained if the Fourier coefficients of different time-modulated elements are known. Therefore, in the following sections, along with the brief description of different timemodulation approaches, the Fourier coefficients of time switching elements under the respective time-modulation scheme are presented.

\subsection{Variable aperture size (VAS)}

This is the first type of time-modulation strategy as reported in [2] where the aperture size of the antenna array is varied with time. The time-modulation principle as discussed in Section 2 falls under this category.

\subsection{Time modulation through pulse shifting}

In VAS time-modulation scheme, only the switch-"on" time duration is considered for deriving the array factor expression. However, when the RF switches are used to commutate the antenna elements in TMAAs, the radiation patterns at center frequency as well as at different harmonics depend not only on the switch-on time duration but also on the switch-"on" and switch-"off" time instants of the array elements $[18,19]$. Thus along with the switch-on time durations as considered in VAS scheme, switch-on and switch-off time instants are also taken as another degree of freedom to control the power pattern in TMAA. For the pulse shifting strategy, periodic switching instants of the $p^{\text {th }}$ element over the modulation period are shown in Figure 8. In this case, both on-time instant $t_{p}^{1}$ and off-time instant $t_{p}^{2}$ can be controlled independently such that individually $t_{p}^{1}$ and $t_{p}^{o n}=t_{p}^{2}-t_{p}^{1}$ should be $\leq T_{m}$. Thus, two situations may occur. The first case is shown in Figure 8(a) where $t_{p}^{1}<t_{p}^{2}$ and $t_{p}^{1}+t_{p}^{o n} \leq T_{m}$. Therefore, the switching function as expressed in Eq. (2) for VAS will be modified and is represented as in Eq. (14): 


$$
U_{p}(t)=\left\{\begin{array}{lc}
1 ; & \left(\eta T_{m}+t_{p}^{1}\right) \leq t \leq\left(\eta T_{m}+t_{p}^{2}\right) \leq(\eta+1) T_{m} \\
0 ; & \text { elsewhere }
\end{array}\right.
$$

Hence, the normalized switch-on time duration, $\tau_{p}$, is given as $\tau_{p}=\frac{t_{p}^{o n}}{T_{m}}=\frac{t_{p}^{2}-t_{p}^{1}}{T_{m}}$. Thus, the pulse shifting strategy reduces to VAS with $t_{p}^{1}=0$.

Another possible situation may appear as shown in Figure 8(b) where $t_{p}^{1}>t_{p}^{2}$ and $t_{p}^{1}+t_{p}^{o n} \geq T_{m}$. Under such situation, the switching operation can be expressed as in Eq. (15):

$$
U_{p}(t)=\left\{\begin{array}{lc}
1 ; & \left(\eta T_{m}+t_{p}^{1}\right) \leq t \leq(\eta+1) T_{m} \text { and }(\eta+1) T_{m} \leq t \leq\left\{(\eta+1) T_{m}+t_{p}^{2}\right\} \\
0 ; & \text { elsewhere }
\end{array}\right.
$$

The complex Fourier coefficient for the pulse shifting strategy at $k^{\text {th }}$ harmonic due to the $p^{\text {th }}$ element under the two cases can be obtained, respectively, as [14].

$$
\text { Pulse shifting (Case 1) } C_{p k}=t_{p}^{o n} \frac{\sin \left(k \pi t_{p}^{o n}\right)}{k \pi t_{p}^{o n}} e^{-j k \pi\left(t_{p}^{o n}+2 t_{p}^{1}\right)}
$$

Pulse shifting (Case 2) $C_{p k}=\frac{1}{k \pi}\left\{\begin{array}{l}\sin \left[k \pi\left(1-t_{p}^{1}\right)\right] e^{-j k \pi\left(1+t_{p}^{1}\right)}+ \\ \sin \left[k \pi\left(t_{p}^{1}+t_{p}^{o n}-T_{m}\right)\right] e^{-j k \pi\left(t_{p}^{1}+t_{p}^{o n}-T_{m}\right)}\end{array}\right\}$

By taking into account the additional degree of freedom, namely, on-time instants of the antenna elements, improved array patterns can be observed. For example, more sideband reduction as compared to VAS approach is obtained when the same array pattern is synthesized at the center frequency [18-19], and electronic beam steering [9] and harmonic beam patterns of different shapes [7, 8] can be realized without phase shifters.

\subsection{Binary optimized time sequence (BOTS)}

In binary optimized time sequence (BOTS), the switch-on time duration of an arbitrary $p^{t h}$ element is divided into $Q$ number of minimal time steps of equal length over a modulation time period $T_{m}$ [20] as shown in Figure 9. The minimal time step, $t^{0}$, is given by

$$
t^{0}=t^{1}=t^{2}=\ldots=t^{q}=\ldots=t^{Q}
$$



Figure 9

Switching function defining binary optimized time sequence (BOTS) strategy. 
The periodic on-off sequence of the set of time steps corresponding to the $p^{\text {th }}$ element is represented by the switching function $U_{p}(t)$. If the on-off status of $q^{\text {th }}$ time step for the $p^{\text {th }}$ element is symbolized with a binary bit, $b_{p}^{q}$, for which "on" status corresponds to $b_{p}^{q}=1$ and that for "off" status $b_{p}^{q}=0$, then the set of time steps for the $p^{\text {th }}$ element is given as $b_{p}^{1} b_{p}^{2} b_{p}^{3} \cdots b_{p}^{Q}$. In order to synthesize the desired pattern, the optimal binary arrangement of the bit patterns, i.e., set of time steps to be under on states and off states, can be found by employing simple genetic algorithm (SGA) [16]. Considering each time step, $b_{p}^{q}$, as a gene, then on-off time sequence of $\mathrm{N}$ array elements represents the chromosome $(\chi)$ of GA and is given as

$$
\chi=b_{1}^{1} b_{1}^{2} b_{1}^{3} \cdots b_{1}^{Q} b_{2}^{1} b_{2}^{2} b_{2}^{3} \cdots b_{2}^{Q} \cdots b_{p}^{1} b_{p}^{2} b_{p}^{3} \cdots b_{p}^{Q} \cdots b_{N}^{1} b_{N}^{2} b_{N}^{3} \cdots b_{N}^{Q}
$$

The complex Fourier coefficient of $p^{\text {th }}$ element at $k^{\text {th }}$ harmonic with the BOTS switching scheme can be obtained as [20]

$$
\text { BOTS : } C_{p k}=\frac{\sin \left(k \pi \tau_{0}\right)}{k \pi} \sum_{q=1}^{Q} b_{p}^{q} e^{-j k \pi \tau_{0}(2 q-1)}
$$

where $\tau_{0}=\frac{t^{0}}{T_{m}}$ is the normalized time step. Thus, by incorporating more number of the degrees of freedom as the optimization variables to the evolutionary algorithm, the radiation pattern characteristics like SLL, SBL, SBR, etc. can be controlled skillfully.

\subsection{Subsectional optimized time steps (SOTS)}

In SOTS-based switching strategy, the time-modulation period $\left(T_{m}\right)$ is divided into a number of subsections with variable lengths [21]. Let us assume that $T_{m}$ is divided into $Q$ number of time steps as shown in Figure 10 for the switching strategy of $p^{\text {th }}$ element of the array. For the $q^{\text {th }}$ time step, the on and off time instants of the switch are denoted by $t_{p}^{q_{o n}}$ and $t_{p}^{q_{\text {off }}}$, respectively, and the resultant ontime duration at $q^{\text {th }}$ step is obtained as $t_{p}^{q}=t_{p}^{q_{\text {off }}}-t_{p}^{q_{\text {on }}}$. Therefore, the periodic time switching pulse $U_{p}(t)$ for the different time steps over a complete modulation period is represented as

$$
U_{p}(t)=\left\{\begin{array}{cc}
1 ; & 0 \leq t_{p}^{q_{o n}} \leq t \leq t_{p}^{q_{o f f}} \leq T_{m}: \quad \forall q \in[1, Q] \\
0 ; & \text { elsewhere }
\end{array}\right.
$$

The Fourier coefficient at the $k^{\text {th }}$ harmonics for the $p^{\text {th }}$ element can be written as

$$
C_{p k}=\sum_{q=1}^{Q} \frac{\left(t_{p}^{q_{\text {off }}}-t_{p}^{q_{\text {on }}}\right)}{T_{m}} \sin c\left(k \omega_{m}\left(t_{p}^{q_{\text {off }}}-t_{p}^{q_{o n}}\right)\right) e^{-j k \omega_{m}\left(t_{p}^{q_{o n}}+t_{p}^{q_{\text {off }}}\right)}
$$

where $\omega_{m}=\frac{2 \pi}{T_{m}}$ denotes the modulation frequency used for the time modulation.

It can be observed that, if the number of subsections $Q$ is 1 , then SOTS is transformed into pulse shifting-based strategy. On the other scenario, if the on-time duration at each step, i.e., the separation between the on and off time instants, becomes multiples of $\frac{T_{m}}{Q}$, then SOTS takes the form of BOTS. So, SOTS-based switching strategy provides more flexibility in the design of optimized time sequences as compared to the other abovementioned switching strategies. However such improved flexibility in synthesizing the pattern is obtained at the cost of some 


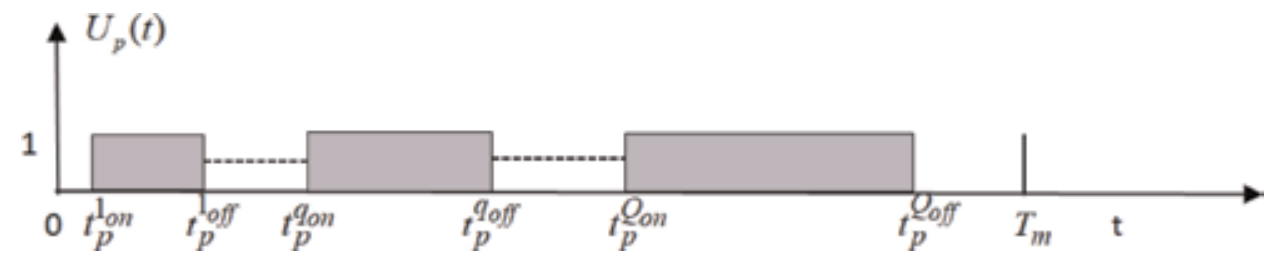

Figure 10.

The schematic of the periodic pulse sequence for SOTS switching strategy of the $p^{\text {th }}$ element of TMLAA.

increased design complexity, because realization of the number of unequal subsections with smaller section over the modulation period needs faster switching operation.

\subsection{Quantized aperture size (QAS)}

In Section 3, the different patterns of desired values of SLL at $f_{0}$ are obtained by making the on-time sequence equal to the Dolph-Chebyshev coefficient of the corresponding patterns. Thus the appropriate set of on-time sequence is required to generate the desired pattern even in uniformly excited TMAAs.

In this section, to generate different patterns in time-modulated antenna arrays (TMAAs) instead of considering continuous value of on-time duration [22], the modulation period is divided into a number of equal steps as in BOTS. However, in BOTS, multiple switching of on-off over the modulation period is considered. Such multiple changes of switching states over the modulation period need fast and complex switching circuit. Unlike BOTS, in this modulation scheme, the on-off states of the switches are assumed to change once over the complete modulation period like VAS. However, the on-off states of the switches are rounded off to the nearest quantization step to obtain quantized on-times (QOTs) of the corresponding elements as shown in Figure 11. In this time-modulation scheme, the time-modulation period, $T_{m}$, is quantized into “ $Q$ " number of discrete levels. At $q^{\text {th }}$ quantization level, the value of $t_{q}$ is given by $q^{*}\left(T_{m} / Q\right)$, where $q=1,2 \ldots Q$. The allowable on-time $t_{p}^{\text {on }}$ of the $p^{\text {th }}$ array elements is taken as $t_{q}$ with $\mathrm{q}=1,2 \ldots \mathrm{Q}$ during each modulation period. Similar to the previously reported VAS time-modulation technique in which the continuous values of on-time durations are optimized to synthesize the desired pattern, this approach is defined as VAS with quantized on-time (VAS-QOT) or simply "quantized aperture size" (QAS) time modulation as the aperture size changes with quantized values of on-time durations of the elements.

\subsection{Nonuniform period modulation (NPM)}

In all of the abovementioned switching strategies, all antenna elements are modulated with the same modulation frequency, $\omega_{m}$, and such time modulation is

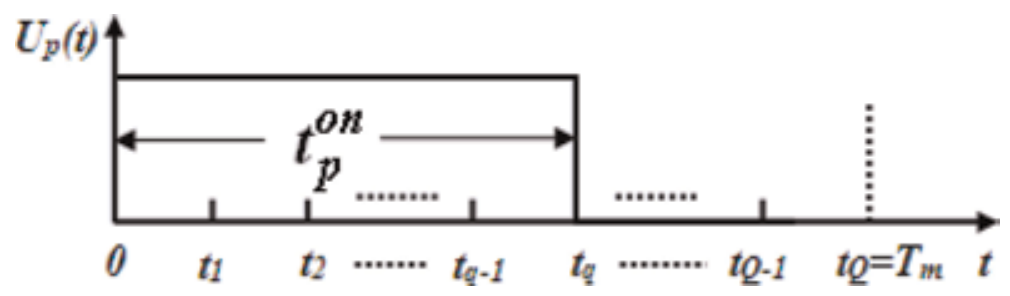

Figure 11.

The proposed time-modulation approach for the quantized on-time of the switches. 


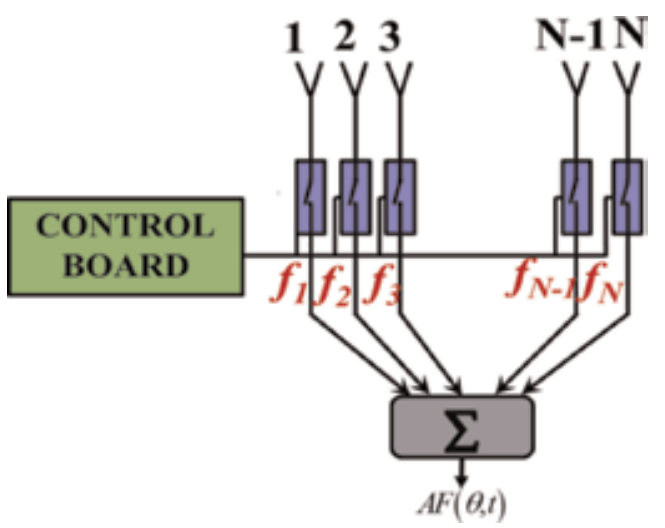

Figure 12.

Time-modulated array architecture with NPM switching strategy where $f_{1} \neq f_{2} \neq \ldots \neq f_{N}$.

termed as uniform period modulation (UPM). Time-modulated antenna array (TMAA) based on UPM is commonly known as uniform TMAA (UTMAA). On the other hand, if the antenna elements of the array are time-modulated with different modulation frequencies as shown in Figure 12, it is defined as time modulation with nonuniform period modulation (NPM), and the corresponding array is defined as nonuniform TMAA (NTMAA) [23-24]. Let us consider that the antenna elements are modulated with different modulation periods $T_{p}: \forall p \in[1, N]$ having modulation frequency $f_{p}=\frac{1}{T_{p}}: \forall p \in[1, N]$, where $T_{p}$ and $f_{p}$, respectively, denote the modulation period and frequency of the $p^{\text {th }}$ antenna element of the array. The periodic switching pulse of the $p^{\text {th }}$ antenna element $U_{p}(t)$ is written as

$$
U_{p}(t)= \begin{cases}1 ; & t_{p}^{1}<t \leq t_{p}^{2} \\ 0 ; & 0<t \leq t_{p}^{1} \text { or } t_{p}^{2}<t \leq T_{p}\end{cases}
$$

where $t_{p}^{1}$ and $t_{p}^{2}$ denote the on-time and off-time instant of the RF switch used to time modulate the $p^{\text {th }}$ antenna element.

And finally, Fourier coefficient at the $k^{\text {th }}$ harmonics for the $p^{\text {th }}$ element is obtained as [24].

$$
C_{p k}=\frac{\sin \left\{k \pi f_{p}\left(t_{p}^{2}-t_{p}^{1}\right)\right\}}{k \pi} e^{-j k \pi f_{p}\left(t_{p}^{2}+t_{p}^{1}\right)}
$$

Let $\tau_{p}=f_{p}\left(t_{p}^{2}-t_{p}^{1}\right)=f_{p} t_{p}^{o n}$ denote the normalized switch-on time duration, and then the corresponding array factor expression is written as

$$
\begin{aligned}
A F(\theta, t)= & e^{j \omega_{0} t} \sum_{p=1}^{N} A_{p} \tau_{p} e^{j\left(\Phi_{p}+\alpha_{p}\right)} \\
& +\sum_{\substack{k=-\infty \\
k \neq 0}}^{\infty} \sum_{p=1}^{N} A_{p} \frac{\sin \left\{k \pi f_{p} t_{p}^{o n}\right\}}{k \pi} e^{-j k \pi f_{p}\left(2 t_{p}^{1}+t_{p}^{o n}\right)} e^{j 2 \pi\left(f_{0}+k f_{p}\right) t} e^{j\left(\Phi_{p}+\alpha_{p}\right)}
\end{aligned}
$$

where $\omega_{0}=2 \pi f_{0}$ denotes the center frequency of the array. 
The first summation indicates that the signals radiated at the center frequency $\omega_{0}$ are accumulated in the space, whereas the second summation is due to the signals radiated at different harmonics. Now, if the modulation frequencies of the antenna elements are selected in such a way that $f_{1}=f_{2}=\ldots=f_{N}=f_{m}$, then the scenario becomes UTMAA, and the term $k f_{p}$ in the second summation becomes $k f_{m}$ that means that the $k^{\text {th }}$-order harmonics of all the elements appeared at the same frequency. The scenario is the same for all other order of harmonics. As a result, radiated signals at the same frequency are accumulated in space, which in turn increases the resultant SBL.

But in the case of NTMAA, the modulation frequencies are selected in such a way that $f_{1} \neq f_{2} \neq \ldots \neq f_{N}$. So, due to different modulation frequencies of different antenna elements, the signals radiated from different harmonics appeared at different frequencies, and the term $k f_{p}$ in the second summation of [25] becomes different for different elements. That means the $k^{\text {th }}$-order harmonics of different elements appear at different frequencies and the scenario is the same for all the other order harmonics. So, unlike UTMAA, the harmonic signals appeared at different frequencies and are distributed in space, which in turn decreases the resultant SBL [23]. Recently, some research works have reported the calculation of the sideband power of NTMAA [24-25], and also the reduction of the sideband power losses using NTMAA is investigated [26].

\section{Synthesis of time-modulated antenna arrays}

\subsection{Pattern synthesis parameters}

In Section 3.3, it is observed that, though the conventional amplitude tapering methods such as Dolph-Chebyshev and Taylor series can be used to obtain the power pattern of the desired SLL with minimum beamwidth at the operating frequency of time-modulated antenna arrays, these methods are not useful to control the undesired power radiated at different sidebands. Similarly, it is also observed that application of the stochastic computational technique, such as GA, for suppressing side lobe level of the center frequency pattern without taking into account the sideband radiation, cannot reduce sideband signal power. Also, the beamwidth of such patterns is unpredictable. The power pattern with low SLL and suppressed sideband is preferred for the different communication systems.

Therefore, the parameters to be considered to synthesize pencil beam pattern in TMAAs as shown in Figure 5 are SLL, FNBW, and SBL. However for the shaped beam pattern such as flattop and cosec squared, in addition to these three parameters, ripple level in the desired shaped region is another parameter to be taken into account. Further, it can be observed that while $S L L$ is reduced, FNBW is increased and $S B L$ is significantly large. In this regard, $S L L, S B L$, and FNBW for pencil beam pattern and $S L L, S B L, F N B W$, and ripple level for synthesizing shaped beam patterns are the conflicting parameters.

\subsection{Multiple objectives}

In Eq. (13), the cost function is defined to synthesize the power pattern with a single objective that is to achieve the desired value of $S L L$ in the synthesized power pattern. Conversely, the synthesized pencil beam patterns at the operating frequency should have reduced SLL along with sufficiently suppressed SBL and narrow beamwidth. Thus, TMAA synthesis problems are multi-objective optimization problems where the multiple objectives are low $S L L$ and narrow beamwidth $(B W)$ 
of the main beam at operating frequency and low value of maximum sideband level $\left(S B L_{\max }\right)$ for synthesizing pencil beam pattern while one more objective is low ripple level for synthesizing shaped beam patterns.

\subsection{The need of evolutionary algorithm}

TMAA synthesis problem is non-convex and nonlinear in nature. A number of numerical techniques as already mentioned-Dolph-Chebyshev and Taylor series [1] —are available to synthesize pencil beam power pattern in conventional antenna arrays (CAAs). Also, some analytical methods are reported to generate shaped beam patterns and phase-only controlled multiple power patterns in CAAs [27, 28, 29]. Durr et al. described a modified Woodward-Lawson technique to design phasedifferentiated multiple pattern antenna arrays with prefixed amplitude distributions [27]. The analytical technique reported in [28] is used to determine the nonlinear phase distribution of linear arrays. A method based on projection approach [29] is proposed to synthesize reconfigurable array antennas of a cosecant ${ }^{2}$ beam and a flattop beam (FTB) by using a common amplitude with phase-only control of analog phase shifters. Though these numerical and analytical techniques can also be applied to determine the nonlinear distributions of dynamic excitation coefficient and phase to synthesize power pattern at operating frequency of TMAAs, such methods have no control on sideband power level. Therefore, the powerful global stochastic optimization tools such as genetic algorithm (GA) [30], differential evolution (DE) [4-5, 31, 32], particle swarm optimization (PSO) [7], simulated annealing (SA) [6, 33], and artificial bee colony (ABC) $[22,34]$ are essentially required to solve such multi-objective TMAA synthesis problems.

\subsection{Cost function with multiple objectives}

Most of the TMAA synthesis problems are solved by applying single-objective optimization method where all the objectives are added with different weighting factors to form a single cost function and the cost function is minimized by employing heuristic evolutionary algorithms. The different stochastic optimization techniques are used with the objective to synthesize desired patterns at the operating frequency by reducing $S L L$ and $S B L$. One of the commonly used techniques to define the cost function of such conflicting multi-objective TMAA synthesis problem is as expressed in Eq. (26):

$$
\psi(\chi)=\sum_{h=0}^{h=V} W_{h} \cdot \mathrm{H}\left(\delta_{h d}-\delta_{h}\right) \cdot\left(\delta_{h d}-\delta_{h}\right)^{\rho}
$$

where $\chi$ is the set of unknown parameters, termed as optimization parameter vector which is to be determined by the used evolutionary algorithm; $\delta_{h}$ with $h=0$, $1,2, \ldots . V$ are the different parameters of the desired patterns; and $\delta_{h d}$ are the desired values of the specific parameters. For example, $\delta_{0}$ is the maximum SLL $\left(S L L_{\max }\right)$ of the pattern at $f_{0}, \delta_{1}$ is the maximum of sideband radiations $\left(S B R_{\max }\right)$ among the first five sidebands, and $\delta_{2}$ represents FNBW. " $W_{h}$ " is the weighting factors for the corresponding terms. $\mathrm{H}($.$) is the Heaviside step function. " \rho$ " is any natural number. It can be seen from Eqs. (13) and (26) that when the obtained values of $\delta_{h}$ are close to their desired values, the cost function value is moving toward zero. Thus, reaching zero value of the cost function confirms that the synthesized pattern satisfies the requirements in terms of the desired values of the intended synthesizing parameters. To illustrate the effectiveness of the cost 
function as defined in Eq. (26), three multi-objective TMAA synthesis problems have been solved in Section 8. It is to be noted that, for different switching techniques, there is a trade-off between sideband level $(S B L)$ and radiated total sideband power $(S R p)$. Reducing the sideband level usually does not guarantee the power reduction. Hence, in that case a power term should be added into cost function.

\subsection{Selection of weighting factors}

In Eq. (26), all the objectives are added with different weighting factors to form a single cost function. In such techniques, it is tedious and difficult to select proper weighting factor for the optimal solution. Improper set of weighting factors strongly effect on achieving the final values of the desired synthesizing parameters and hence on the performance of the optimization algorithm. Generally, some selected best results are presented without mentioning such difficulties. However, these values of the weighting factors are obtained by trial and error method [4]. Though multi-objective evolutionary algorithm (MOEA) $[35,36]$ can be used to solve such problems, the researchers are not comfortable with it as it has been used rarely as compared to single-objective optimization approaches.

\subsection{Evolutionary algorithms}

It is already discussed that time-modulated antenna array synthesis problems are non-convex as well as nonlinear. Therefore, stochastic, global computational techniques are required to solve such problems. In this regard, different populationbased global searching techniques such as DE, SA, GA, PSO, ABC, and multiobjective evolutionary algorithm (MOEA) have been applied successfully to synthesize the desired pattern at the center frequency by suppressing sideband radiation to satisfactorily low levels. However, here the working principle of $\mathrm{ABC}$ and its implementation have been presented, and a novel approach to synthesize TMAA is discussed.

\section{A quantized time modulator (QTM) to synthesize different patterns in TMAAs}

In Section 4.5, the quantized aperture size (QAS) time modulation or variable aperture size with quantize on-time duration has been explained. In this section first to realize such time-modulation approach, a time modulator, namely, quantized time modulator (QTM), is presented. Then it is shown that though the quantized on-time duration has been used, however, by selecting a suitable number of quantization levels, the effect of quantization errors on the synthesized patterns can be reduced. In order to select the best possible set of quantized on-time values, the potentiality of artificial bee colony algorithm $(\mathrm{ABC})$ has been exploited as the global searching algorithm. Thus, for the desired patterns, $A B C$ finds the optimum set of unknown parameter values from the discrete search space of QOT. The synthesized results as obtained by using this quantized on-time are compared with that achieved by using continuous search space of on-time $[6,33]$. Finally, considering the discrete search space of QOT, a low side lobe level (SLL) flattop pattern with low dynamic range ratio (DRR) is synthesized by utilizing a fully digitally controlled QTM. The major advantage of this approach is that by implementing the "time modulator" either as a discrete component on a printed circuit board or in an integrated circuit (IC), it can generate different patterns in the TMAA system. 
Pattern Synthesis in Time-Modulated Arrays Using Heuristic Approach DOI: http://dx.doi.org/10.5772/intechopen.89479

\subsection{Quantized time modulator (QTM)}

For appropriate switching operation at $p^{\text {th }}$ element, a current pulse with a pulse width of $t_{p}^{o n}$ is required [2]. The proposed scheme for the periodical switching of the antenna array elements is shown in Figure 13. The QTM has two parts, namely, quantized pulse generator (QPG) and pulse width selector (PWS). In QPG, the consecutive tap delay output line, $\mathrm{TAP}_{i}$ with $i=1,2, . . \mathrm{Q}$, introduces an equal delay of " $T_{m} / Q$." The pulse output from the pulse generator (PG) is used to set the

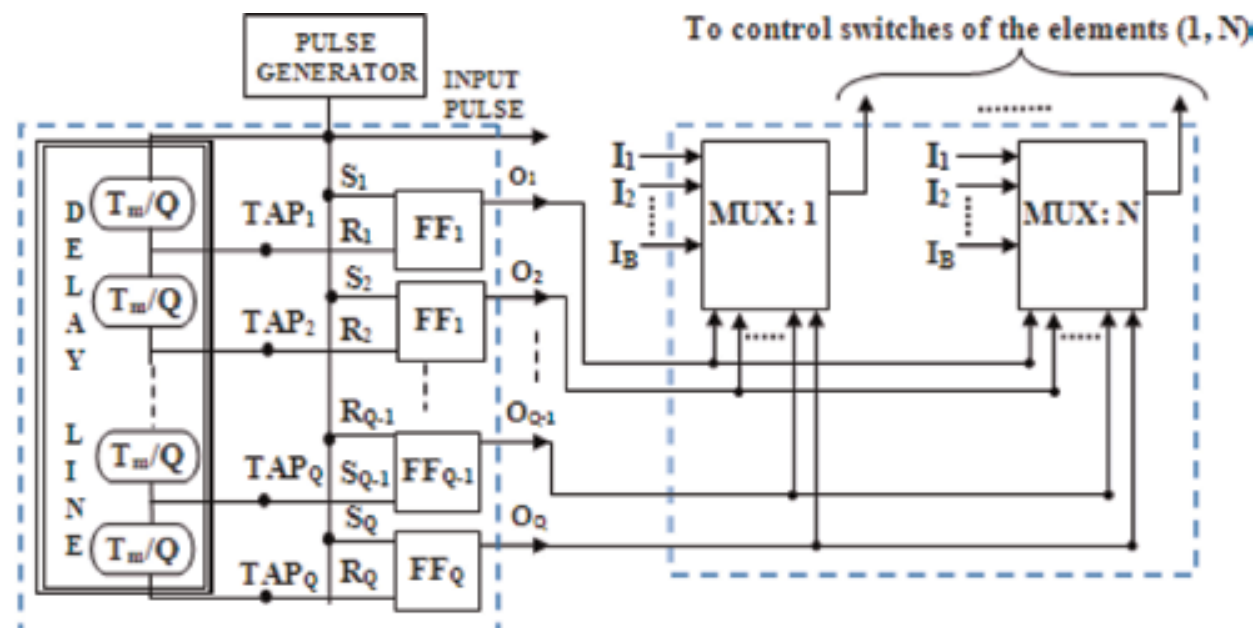

Quantized Pulse Generator (QPG)

Pulse-Width Selector (PWS)

Figure 13.

The proposed quantized time modulator (QTM).

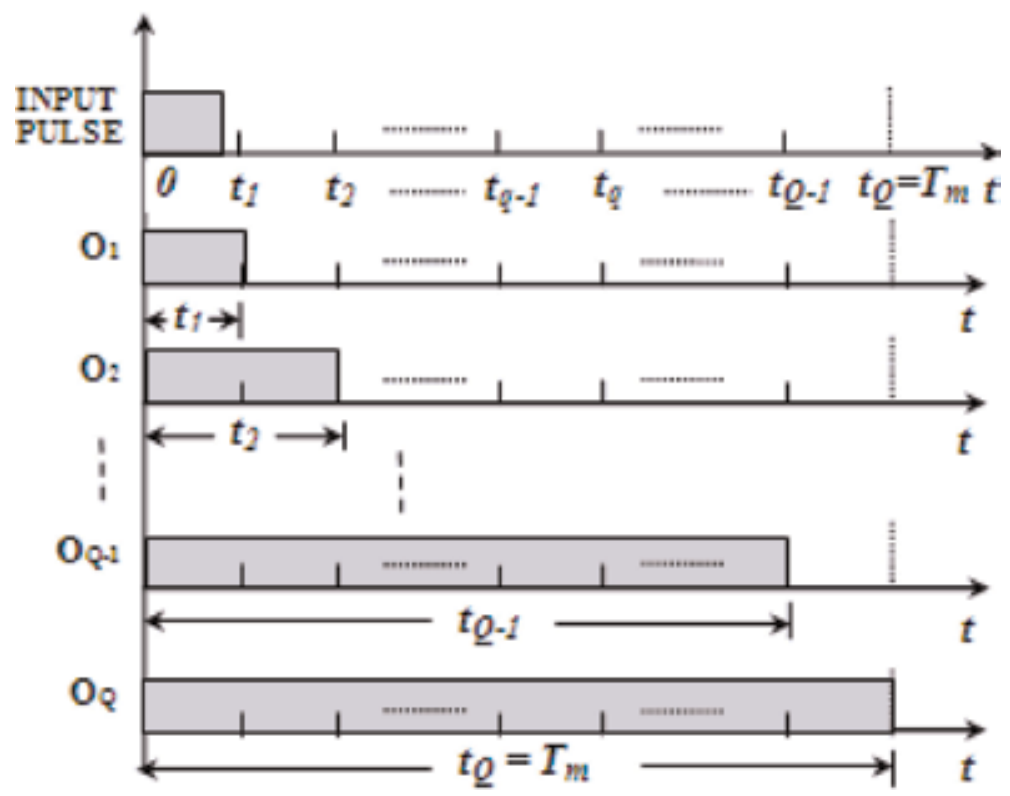

Figure 14.

The wave form of the input and output pulses of different pulse widths that can be obtained at the outputs $O_{q} \forall q \in(1, \ldots, Q)$ in Figure 13. 
flip-flop (FFs) outputs to logic level 1, whereas the delayed pulses from the corresponding tap outputs of the delay line are applied to reset the flip-flop outputs to logic level $\mathbf{0}$. To avoid the simultaneous appearance of PG output and its delayed version at the $\mathrm{S}$ and $\mathrm{R}$ inputs of $i^{\text {th }}$ flip-flop, respectively, the pulse width less than $\mathrm{Tm} / \mathrm{Q}$ can be used. The current waveforms of the input pulse applied to the set (S) inputs of the flip-flops and output pulses appeared at the outputs $O_{q}$ with $q=1,2$, ... $Q$ of different flip-flops as shown in Figure 14. Therefore, the QPG, consisting of a pulse generator, simple tapped delay line, and flip-flops, provides the required current pulses with quantized values of $t_{p}^{\text {on }}$.

One of the most important features in TMAAs is to reconfigure different antenna patterns just by changing the on-time sequence across each element. Such a feature can easily be obtained in the proposed QTM employing PWS. The PWS consists of $N$ number of $(Q \times 1)$ multiplexers and their outputs that are used to modulate antenna element using the quantized values of $t_{p}^{o n}$. With appropriate bit combination at the select inputs $I_{0}, I_{1}, \ldots I_{B}$ of the multiplexers, one of the quantized pulses at the output of QPG is selected to time modulate the corresponding antenna element. Thus, just by using the appropriate combination of the select lines of multiplexers, it is very easy to reconfigure different patterns.

\section{Artificial bee colony $(\mathrm{ABC})$ algorithm}

Karaboga [37] introduced the artificial bee colony (ABC) algorithm to simulate intelligent food foraging behavior of the honeybee swarm. The $\mathrm{ABC}$ algorithm shows excellent performance for optimizing multivariable functions as compared to other similar algorithms like genetic algorithm (GA), differential evolution (DE), and particle swarm optimization (PSO). ABC is a robust search and optimization algorithm with relatively fewer control parameters [38]. Although GA is extensively used due to its efficiency to solve the optimization problems with binary/discrete variables, it requires high computational time as well as high memory consumption to store unnecessary binary data during the conversion of a real number to binary and vice versa. The decoding method as applied in $A B C$ algorithm requires one-line MATLAB code which directly quantizes continuous values of the variables by rounding off them. The food foraging behavior of real bees and the implementation of the algorithm have been briefly discussed in the following section.

\subsection{Food foraging behavior of real bees}

The constituents of the food foraging systems are the unemployed bees (UBs) and the employed bees (EBs) in a beehive and food sources (FSs) in their surroundings. Initially, all the bees are unemployed, and after they find a rich food source, they become employed. UBs are categorized into scout bees ( $S B \mathrm{~s}$ ) and onlooker bees $(O B s)$. The food foraging process is initiated when the $S B s$ start to explore the rich food source randomly from any location by moving toward any direction of the search space. When $S B s$ find a rich food source, it becomes an $E B$ and returns to the hive to attract other bees by performing a special dance known as the waggle dance. Depending on the quality of the food source, the $E B s$ recruit some bees to extract nectar from the source. The EBs abandon the current food source when the nectar of the source is finished and becomes scout bees (SBs). However, in the dancing area, $O B$ s examine the quality and quantity of the food sources with the information provided by the EBs, and after examinations EBs select a food source. Thus during the food foraging process, exploration is carried out by $S B$ s, and 
exploitation is carried out by $E B s$ and $O B$ s. Due to the presence of both exploration and exploitation, $\mathrm{ABC}$ becomes a robust search and optimization algorithm. It is to be noted that the objective of the bees in $A B C$ is to find out the location of the best possible food sources within the search space. Hence, the possible locations of the food sources are the possible solutions to this process. But in other swarm intelligence algorithms, e.g., particle swarm optimization (PSO), the locations of the individual agents are the possible solution within the search space. It is assumed that the number of employed bees $(N E)$ and number of onlooker bees are equal in the colony and also these are equal to the number food sources $(F N)$.

\subsection{Implementation of $\mathrm{ABC}$}

In the following steps, the real bee colony behavior into the problem space is implemented:

a. Specifying objective: The objective is to synthesize far-field patterns at $f_{0}$ by simultaneously minimizing $S L L, S B L_{\max }$, and first null beamwidth (FNBW) or ripple $(R)$.

b. Parameters to be optimized: Depending on the requirement in an array synthesis problem, suitable independent parameters are chosen as the optimization parameter vector $\chi$. The number of parameters in $\chi$ represents the dimension $(D)$ of the specific optimization problem.

c. Defining the cost function: According to the design parameters discussed above and multiple objectives of the synthesis problem, the cost function is defined as

$$
\psi(\chi)=\sum_{h=0}^{h=2} W_{h} \cdot \mathrm{H}\left(\left|\delta_{h d}\right|-\left|\delta_{h}\right|\right) \cdot\left(\delta_{h d}-\delta_{h}\right)^{2}
$$

where $\delta_{h}$ with $h=0,1$, and 2 are the instantaneous values of different parameters of the desired patterns, while $\delta_{h d}$ is the desired values of the specific parameters. For all examples as considered in Section $8, \delta_{0}$ is the maximum $S L L\left(S L L_{\text {max }}\right.$ ) of the pattern at $f_{0}$ and $\delta_{1}$ is the value of $S B L_{\max }$ among the first five sidebands. But, for the first two examples, $\delta_{2}$ represents $F N B W$, and, for the third case, it is the ripple level of the flattop pattern for which the positions of $\delta_{h d}$ and $\delta_{h}$ are interchanged in the Heaviside step function $\mathrm{H}(\cdot)$. " $W_{h}$ " is the weighting factor for the corresponding terms. The cost function $\psi$ in Eq. (27) depends on " $D$," the independent parameters of optimization parameter vector $\chi$. A possible set of the parameter values may be considered as a point in the search space of $D$ dimensional coordinate system. In $\mathrm{ABC}$, the cost function $\psi$ of the optimization problem has resembled with the food sources of the bees and each possible point as its location. The solutions of the optimization problem represent locations of the food sources, whereas the corresponding value of cost function $\psi$ due to each point in its solution set is considered as the quality of the food source:

d. Initialization: The possible solution, $\chi_{i}$, where $i=1,2 \ldots F N$, of an arbitrary number of food sources is generated randomly within the search space. With $F N$ possible locations, each with $D$ dimension is expressed in terms of a $[F N \times D]$ matrix.

e. Evaluating the quality of the food source: For all the possible solutions, the values of $\psi$ and the corresponding fitness values, $\mu_{i}$, are evaluated. 
f. Employed bees' stage: The greedy nature of the employed bees $\left(E B_{s}\right)$ is incorporated, and the new sources $\left(s_{i}\right)$ surrounding its neighborhood are generated as follows:

$$
s_{i j}=\chi_{i j}+\mathfrak{R}_{i j}\left(\chi_{i j}-\chi_{z j}\right)
$$

where $j \in\{1,2, \ldots, D\}$ and $z \in\{1,2, \ldots, F N\}$ are randomly selected column and row indexes of the position matrix and ${ }_{i j}$ is any randomly generated number through $[-1,1]$. When any parameter of the new solution crosses its lower limit, it is replaced by its predetermined minimum value $\left(\chi_{\min }^{j}\right)$ and for the upper limit by its maximum value $\left(\chi_{\max }^{j}\right)$. If, for a new solution, the value of $\psi$ is less than the corresponding old solution, the old is replaced by the new one.

g. Onlooker bees'stage: The quality of the food source is represented by the fitness value, $\mu_{i}$, of the cost function, and onlooker bees select the new source by means of the probability, $\xi_{i}$, in terms of the fitness value, determined by

$$
\xi_{i}=0.9\left(\frac{\mu_{i}}{\mu_{\max }}\right)+0.1
$$

where $\mu_{\max }$ is the maximum fitness value among the current possible solutions. Like employed bees (EBs), the greedy selection is also applicable to onlooker bees (OBs).

h. Scout bees' stage: In this stage, the abandonment of a food source by the employed bees is simulated. If the fitness value of the cost function is not

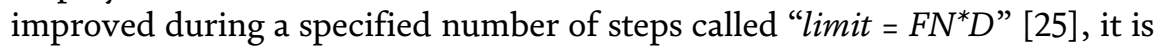
ignored, and the parameter, $q_{i}^{j}$, for the new solution is provided randomly through the whole search space by Eq. (30):

$$
q_{i}^{j}=\chi_{\min }^{j}+\operatorname{rand}(0,1)\left(\chi_{\max }^{\mathrm{j}}-\chi_{\min }^{\mathrm{j}}\right)
$$

i. Remembering the best solution: The overall new best solution as mentioned in the steps "e- $\mathrm{h}$ " replaces the previous best, and the value is then stored.

j. Stopping criterion: Steps " $(e)$ " to “ $(i)$ ” are repeated until the cost function converges to the desired value or a predetermined value of maximizing the number of cycles (MNC).

\section{Design examples and discussions}

The VAS-based synthesis problems that have been reported in $[6,33]$ are considered at first, and the QAS-based time-modulation approach is applied to realize the patterns. Here, the modulation period $T_{m}$ is quantized in 10 equal discrete levels, i.e., $Q=10$. Hence, the discrete search space for the optimization problem $\left(\tau_{p}\right)$ becomes $\{0.1,0.2,0.3,0.4,0.5,0.6,0.7,0.8,0.9,1\}$.

Example 1: A 30-element UE TMLAA is placed along the $\mathrm{x}$-axis with one element at the origin, and a uniform inter-element spacing of $0.7 \lambda$ is considered. It is desirable in practice for such an array to feed with $\left\{A_{p}\right\}=1$ and $\left\{\phi_{p}\right\}=0$. Here, 
$\chi=\left\{\tau_{p}\right\}$ is taken as the optimization parameter vector. The control parameters of $\mathrm{ABC}$ such as $E N=30$, limit $=900$ (limit $=E N^{*} D$ ), and $M N C=700$ are selected as per the guidelines given in [38]. $W_{1}, W_{2}$, and $W_{3}$ are selected as 2,1 , and 1 , respectively. In Eq. (27), $\delta_{1 d}, \delta_{2 d}$, and $\delta_{3 d}$ are set as $-20,-30$, and $7 \mathrm{~dB}$, respectively. The ABC optimized far-field power pattern with side lobe level (SLL) of $-20.15 \mathrm{~dB}$, $F N B W$ of $6.86^{\circ}$, and sideband levels ( $S B L s$ ) at the first two sidebands as $S B L_{1}=-30.78 \mathrm{~dB}$ and $S B L_{2}=-31.63 \mathrm{~dB}$, respectively, is shown in Figure 15.

Table 2 contains the ABC optimized values of $\tau_{p}$ of the elements used to obtain Figure 15. As compared to [6], $S L L_{\max }$ and $S B L_{\max }$ are improved by a factor of 0.1 and $0.7 \mathrm{~dB}$, respectively, in the proposed work. The total sideband power is calculated by using either of the expressions derived in [14] or [39] and found to be $4.83 \%$ of the total power which is quite higher than $3.89 \%$ and $3.57 \%$ as reported in

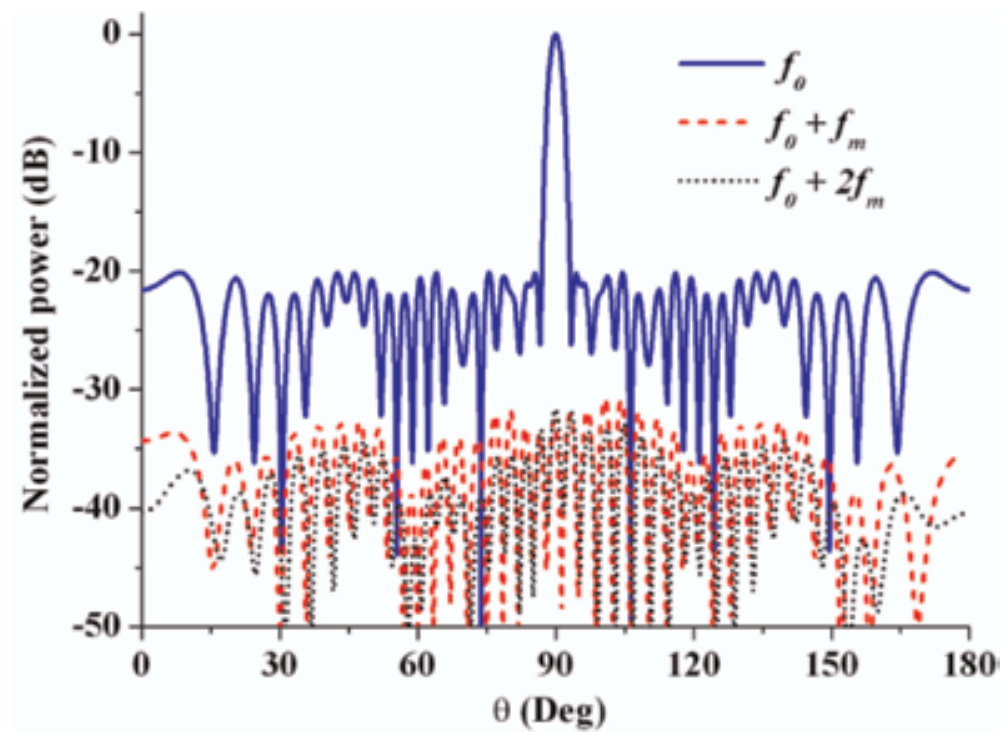

Figure 15.

$A B C$ optimized power pattern obtained by using the discrete value of $\tau_{p}$ of Table 2.

\begin{tabular}{ll}
\hline Element numbers $(\mathbf{p})$ & $\tau_{\boldsymbol{p}}$ \\
\hline 1 & 1 \\
\hline 2 & 0.30 \\
\hline 3 & 0.10 \\
\hline $4-22$ & 1 \\
\hline 23 & 0.90 \\
\hline 24 & 0.90 \\
\hline 25 & 0.10 \\
\hline 26 & 0.10 \\
\hline 27 & 0.10 \\
\hline 28 & 0.90 \\
\hline 29 & 0.10 \\
\hline
\end{tabular}

Table 2.

Optimum discrete values of $\tau_{p}$ for the power pattern of Figure 15. 
the work of $[6,40]$, respectively. However, the method as proposed in [40] may be utilized to reduce the waste of power in the form of sideband radiations.

Example 2: In the second example, the synthesis problem as discussed in [33] is considered. From the list of static and dynamic excitations of one-half of the linear arrays as presented in Table 3, Ref. [33], it was found that out of the five edge elements, only three are time-modulated to synthesize the sum pattern, whereas, for the difference pattern, time modulation is applied only on four center elements. In this work, to synthesize the sum and difference pattern, the proposed method is applied in the following way. For the UE TMLAA, the sum pattern is synthesized by taking the discrete $\tau_{p}$ values of five edge elements (in one-half of the array) as " $\chi$." In order to compare the $\mathrm{ABC}$ optimized results with those of SA, during optimization, the three lower values of $\tau_{p}$ are rounded off to their nearest quantization levels, whereas the higher two $\tau_{p}$ values are kept to 1 so that the ABC optimized pattern is obtained by time modulating the same number of (i.e., three) elements as observed in SA. However, to synthesize the difference pattern, perturbation of discrete $\tau_{p}$ values of four center elements are considered. In Eq. (27), the same values of $\delta_{h d}$ 's as used in Example 1 are set. Figures 16 and 17 show the ABC optimized sum and difference patterns, respectively. For optimizing the sum and difference pattern with $N E=30$ and limit $=450$, the $\mathrm{ABC}$ takes only 23 and 5 iterations, respectively (refer to Figure 18). The corresponding optimum discrete values of $\tau_{p}$ are shown in

\begin{tabular}{|c|c|c|c|c|c|c|c|c|c|c|c|}
\hline \multicolumn{2}{|c|}{ Element numbers } & \multirow{2}{*}{$\begin{array}{c}1 \& \\
30 \\
1\end{array}$} & \multirow{2}{*}{$\begin{array}{c}2 \& \\
29 \\
1\end{array}$} & \multirow{2}{*}{$\begin{array}{c}3 \& \\
28 \\
0.2\end{array}$} & \multirow{2}{*}{$\begin{array}{c}48 \\
27 \\
0.9\end{array}$} & \multirow{2}{*}{$\begin{array}{c}5 \& \\
26 \\
0.1\end{array}$} & \multirow{2}{*}{$\begin{array}{c}6-11 \& \\
25-20 \\
1\end{array}$} & \multirow{2}{*}{$\begin{array}{c}12 \& \\
19 \\
1\end{array}$} & \multirow{2}{*}{$\begin{array}{c}13 \& \\
18 \\
1\end{array}$} & \multirow{2}{*}{$\begin{array}{c}14 \& \\
17 \\
1\end{array}$} & \multirow{2}{*}{$\begin{array}{c}15 \& \\
16\end{array}$} \\
\hline$\tau_{p}$ & Sum pattern & & & & & & & & & & \\
\hline & $\begin{array}{l}\text { Difference } \\
\text { pattern }\end{array}$ & 1 & 1 & 1 & 1 & 1 & 1 & 0.1 & 0.9 & 0.3 & 0.1 \\
\hline
\end{tabular}

Table 3 .

Optimum discrete values of $\tau_{p}$ of $A B C$ optimized sum and difference pattern, as shown in Figures 12 and 13.

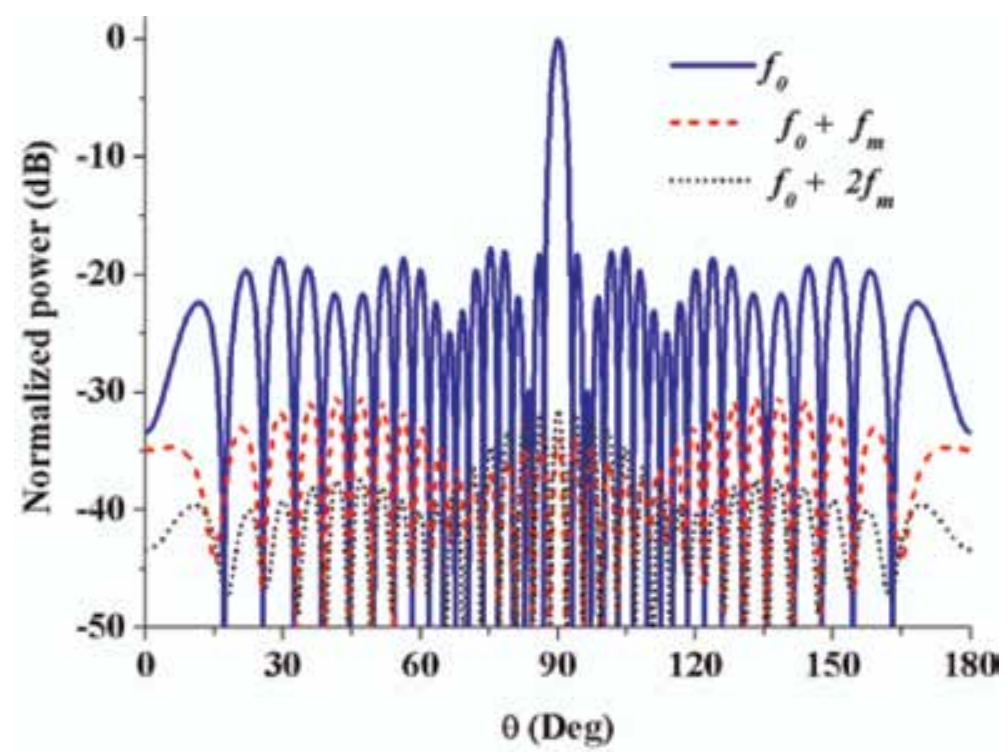

Figure 16.

$A B C$ optimized sum pattern as obtained by time modulating the same percentage (20\%) of elements as in [33]. $S L L$ and $S B L_{\text {max }}$ of the pattern are obtained as -17.87 and $-31.44 \mathrm{~dB}$, respectively. 
Table 3. It can be observed that the sum and difference pattern is obtained by time modulating the same number of elements as found in [33]. As compared to [33], $S L L_{\text {max }}$ and $S B L_{\text {max }}$ of the sum pattern are improved by 2.03 and $1.5 \mathrm{~dB}$, respectively. In case of difference pattern, the $\mathrm{SBL}_{\max }$ is reduced by $2.37 \mathrm{~dB}$ with only $0.37 \mathrm{~dB}$ rise in $S L L$. Also, for both the sum and difference patterns, the amount of sideband power is found to be $3.35 \%$ and $4.69 \%$ of the total power which are $4.30 \%$ and $5.45 \%$ in the respective patterns of [33]. The FNBW of ABC optimized sum

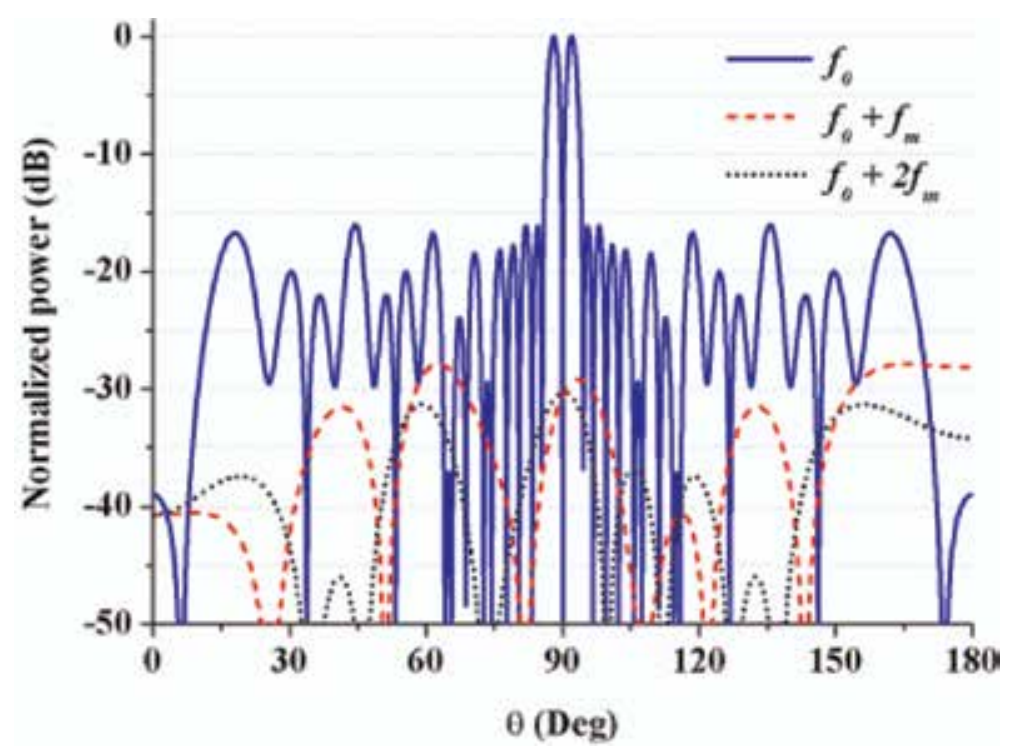

Figure 17.

$A B C$ optimized difference pattern as obtained by time modulating the same percentage (26.7\%) of elements as in [33]. SLL and $S B L_{\max }$ of the pattern are obtained as -16.05 and $-31.44 \mathrm{~dB}$, respectively.

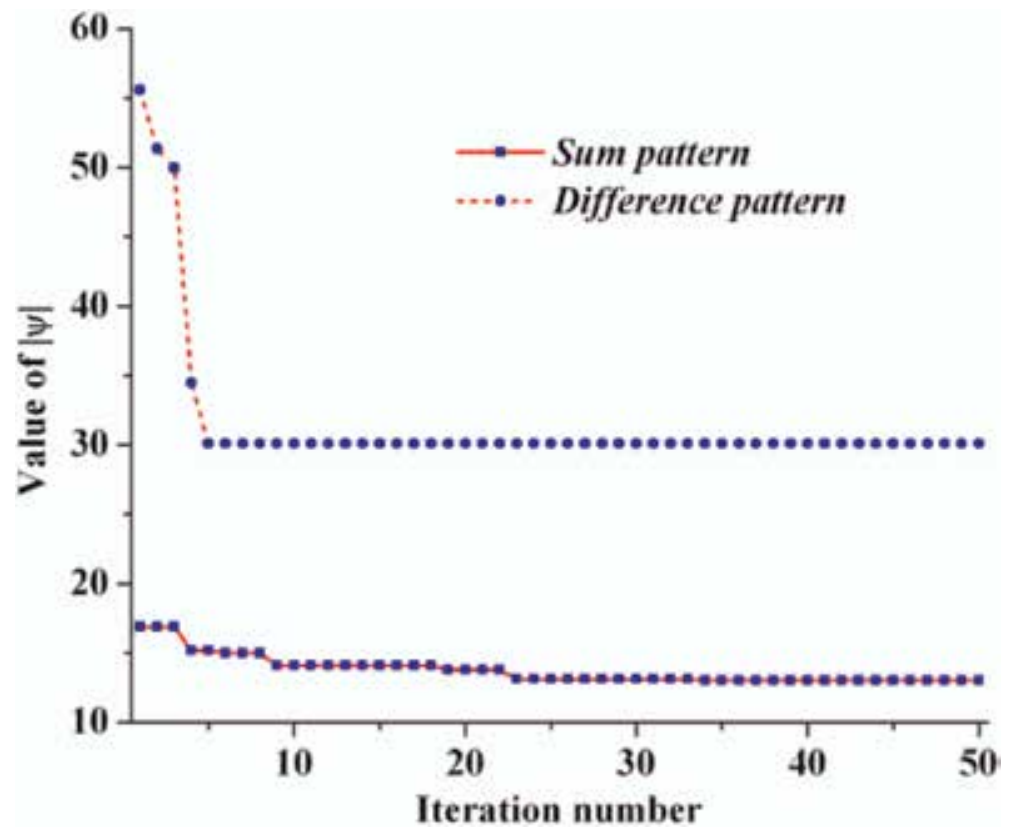

Figure 18.

Convergence characteristics of $A B C$ for the synthesized sum and difference patterns of Figures 5 and $\mathbf{6}$. 


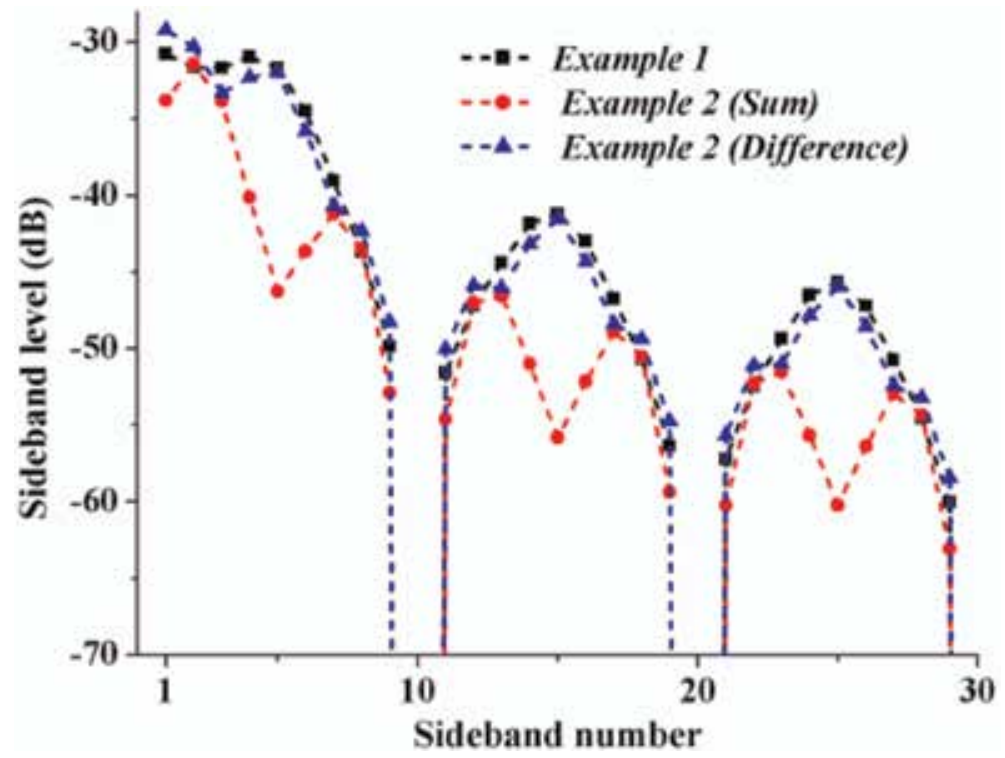

Figure 19.

Sideband levels of the first 30 sidebands for the different patterns in examples 1 and 2.

pattern and difference pattern was found as 6.12 and $4.56^{\circ}$, respectively, which are quite comparable to 5.88 and $4.59^{\circ}$ as for the patterns in [33].

Figure 19 shows $S B L$ s of the first 30 sidebands for the synthesized patterns as considered in Example 1 and Example 2. It can be observed that at the higher sidebands also, the $S B L$ s are below $S B L_{\text {max }}$. Further observation shows that the no radiation is produced at $10^{\text {th }}, 20^{\text {th }}$, and $30^{\text {th }}$ sideband with quantized values of $\tau_{p}$ as at these harmonics the array factor expression becomes zero for all elements.

Example 3: In this example, it is shown that the same time modulator can also be used to synthesize a flattop pattern. Accordingly, a symmetrical TMLA with element number $N=20$ and inter-element spacing $d_{0}=0.5 \lambda$ is considered. Here, the objective is to synthesize a flattop pattern in the broadside direction with digitally controlled static excitation amplitudes and phases by using five digital attenuators and phase shifters. A flattop pattern with a beamwidth of $30^{\circ}$, maximum ripple level $\left(R_{\max }\right)$ at the flat region of less than $1 \mathrm{~dB}$, and transition width of $8^{\circ}$ is selected as the target pattern. Although such pattern with more stringent design specification is reported in [6], analog attenuators and phase shifters are required. Due to symmetry, the dimension of the parameter vector $\chi=\left\{A_{p}, \phi_{p}, \tau_{p}\right\}$ becomes 30 . During optimization, $A_{p}$ and $\phi_{p} \forall p \in(1, \ldots, N)$ are perturbed within the search range of $(0.2-1)$ and $(-180$ to +180$)$ with step sizes of $0.5 / 2^{5}$ and $360 / 2^{5}$, respectively. The number of quantization states for $\tau_{p}$ is selected as 20 . In Eq. (27), both $\delta_{1 d}$ and $\delta_{2 d}$ are selected as $-30 \mathrm{~dB}$, while $\delta_{3 d}$ is set to $1 \mathrm{~dB}$. Setting $F N=150$, the $\mathrm{ABC}$ parameters are obtained as in [38]. ABC converges after 2000 iterations, while the weighting factors are selected as $W_{1}=2 ; W_{2}=1$; and $W_{3}=5$. The ABC optimized 3D space pattern at fundamental frequency along with the first 30 sidebands is shown in Figure 20. Table 4 contains the corresponding discrete values of $A_{p}, \phi_{p}$, and $\tau_{p}$. The flattop pattern in Figure 20 is obtained with $S L L, S B L_{\max }$, and $R_{\max }$ of -29.31 , -29.9 , and $1.22 \mathrm{~dB}$, respectively. The absolute value of $R_{\max }$ is measured in the region of $75 \leq \theta \leq 105^{\circ}$. Hence, only $0.22 \mathrm{~dB}$ higher values of $R_{\max }$ are obtained by satisfying other design specification of the pattern. Also, it is observed that no such improvement in the pattern is obtained when the continuous value of $\tau_{p}$ is used to 


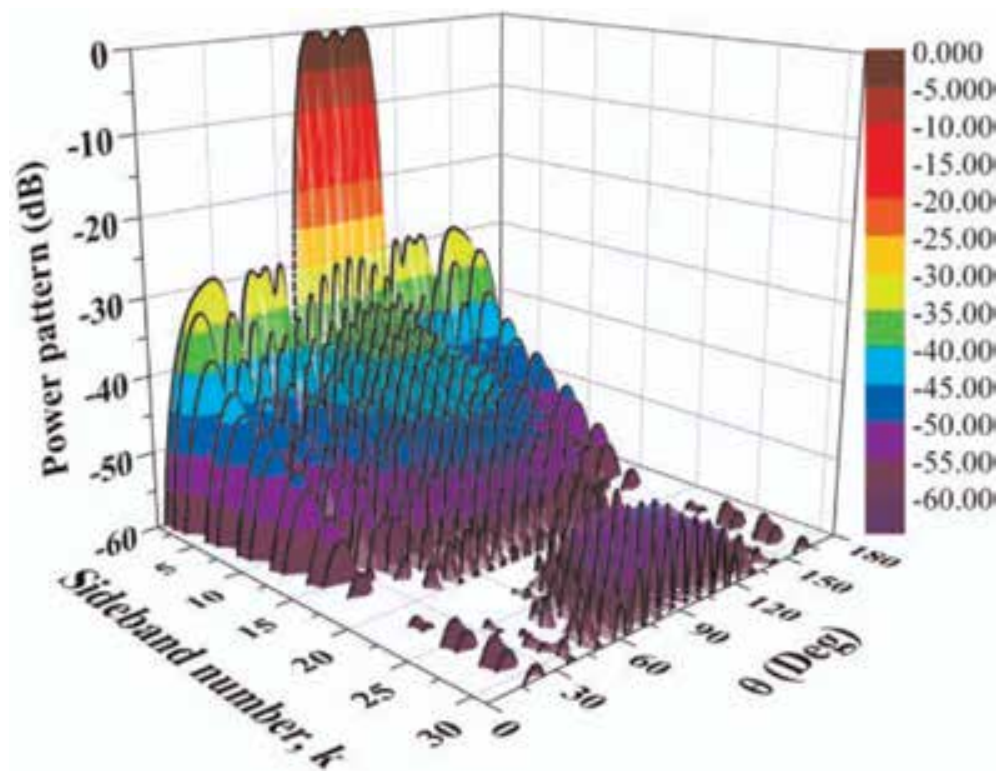

Figure 20.

$A B C$ optimized space pattern at $f_{o}$ and the first 30 sidebands. At $f_{o}$, the flattop pattern is obtained with SLL, $S B L_{\max }$, and $R_{\max }$ of $-29.31,-29.9$, and $1.22 \mathrm{~dB}$, respectively.

\begin{tabular}{cccc}
\hline Element numbers $(\mathbf{p})$ & Normalized on-time, $\tau_{p}$ & \multicolumn{2}{c}{ Discrete values of excitation } \\
\cline { 3 - 4 } & & Amplitude, $A_{p}$ & Phase, $\boldsymbol{\phi}_{\boldsymbol{p}}$ \\
\hline $1 \& 20$ & 0.65 & 0.200 & -33.75 \\
\hline $2 \& 19$ & 0.95 & 0.325 & -22.50 \\
\hline $3 \& 18$ & 0.95 & 0.475 & 0 \\
\hline $4 \& 17$ & 1 & 0.525 & 33.75 \\
\hline $5 \& 16$ & 1 & 0.675 & 67.50 \\
\hline $6 \& 15$ & 1 & 0.975 & 90 \\
\hline $7 \& 14$ & 1 & 1 & 112.50 \\
\hline $8 \& 13$ & 1 & 0.800 & 135 \\
\hline $9 \& 12$ & 1 & 0.600 & -180 \\
\hline $10 \& 11$ & 1 & 0.700 & -146.25 \\
\hline
\end{tabular}

Table 4

Optimum discrete values of $A_{p}, \phi_{p}$, and $\tau_{p}$ for the flattop power pattern of Figure 20.

synthesize the pattern. However, with $Q=10$, almost the same pattern is obtained with $R_{\max }$ of $1.80 \mathrm{~dB}$.

In the continuous search space of VAS time-modulation method [2, 3-6], the ontime duration of array elements can be of any value between 0 and $T_{m}$. In [2], for each time-modulated elements, the current pulse required with pulse width over the range of $\left(0.1 T_{m}<t_{p}^{o n}<0.9 T_{m}\right)$ is obtained by using the RF switches with individually controlled switching circuits. Other time-modulation schemes such as BOTS [30] and SOTS [21] need a complex programmable logic device (CPLD) for controlling the "on-off" timing of the connected switches. The continuous values of 
on-time of elements can be controlled by using CPLD accurately [41, 42], but to synthesize a new antenna pattern, by realizing a new set of on-time sequence, the CPLD must be reprogrammed by completely erasing the previous set of on-time values. In contrast, the proposed VAS-QOT needs a simple circuitry as shown in Figure 13, where the new set of on-time sequences according to the need can be obtained simply by altering the appropriate binary input sequence to the selected inputs of the multiplexers. Thus, by fabricating the QTM in integrated circuit (IC) or by using some discrete components on a printed circuit board (PCB), TMAA switching can be done easily.

\section{Conclusions}

Introduction of the additional degree of freedom "time" provides flexibility in synthesizing antenna array patterns and overcomes the shortfalls of realizing the patterns through conventional array synthesis methods. Among the different timemodulation strategies, QAS can be realized through a simple digital circuit consisting of a pulse generator, simple tapped delay line with equal delay at each tap output, flip-flops, and multiplexers. This circuit can be implemented in either an integrated circuit (IC) form or in a printed circuit board and can be used as a discrete component to generate different patterns. However, as far as the nonuniform period modulation is concerned, the function of the quantized time modulator (QTM) circuit needs to be investigated, specifically to time modulate the elements with multiple frequencies which need accommodation of multiple PLLs in the circuit for the multiple frequencies. Regarding other time-modulation approaches, complexity in the switching circuit increases as per the sequence, VAS, pulse shifting, BOTS, SOTS, and NPM, respectively, while their performance in synthesizing low SLL power patterns with suppressed SBL follows the reverse order. Thus, for a time-modulation approach, the improved performance in terms of the capability of synthesizing low side lobe power patterns by suppressing harmonic signal level is obtained at the cost of complex switching mechanism. However, due to the advancement in the semiconductor technology, availability of highspeed semiconductor switches makes it possible to realize such complex switching mechanism by writing simple program code in complex programmable logic devices (CPLDs).

In all the time-modulation approaches except NPM, for the desired power pattern, optimization algorithm is required to determine the proper set of on-time sequence. The construction of suitable cost function with multiple objectives such as narrow beamwidth; low values of SLL and SBL, etc.; and the selection of corresponding weighting factors plays an important role to achieve the best possible power patterns. This chapter gives a brief fundamental insight toward all this issues.

\section{Acknowledgements}

This work is financially supported by the Ministry of Electronics and Information Technology (MeitY), Govt. of India, under Visvesvaraya Young Faculty Fellowship of Visvesvaraya Ph.D. scheme (Grant No. PhD-MLA-4(29)/ 2015-2016) and DST-SERB project ref. file number EEQ/2016/00836, dated January 17, 2017. 
Pattern Synthesis in Time-Modulated Arrays Using Heuristic Approach

DOI: http://dx.doi.org/10.5772/intechopen.89479

\section{Author details}

Sujit Kumar Mandal*, Ananya Mukherjee, Sujoy Mandal and Tanmoy Das Microwave and Antenna Research Laboratory, Department of Electronics and Communication Engineering, National Institute of Technology Durgapur, Durgapur, West Bengal, India

*Address all correspondence to: skmandal2006@gmail.com

\section{IntechOpen}

(C) 2019 The Author(s). Licensee IntechOpen. This chapter is distributed under the terms of the Creative Commons Attribution License (http://creativecommons.org/licenses/ by/3.0), which permits unrestricted use, distribution, and reproduction in any medium, provided the original work is properly cited. (c) BY 


\section{References}

[1] Balanis CA. Antenna Theory Analysis and Design. 3rd ed. New Delhi: A John Wiley and Sons, Inc. Publication; 2010

[2] Kummer WH, Villeneuve AT, Fong TS, et al. Ultra-low side-lobes from time-modulated arrays. IEEE Transactions on Antennas and Propagation. 1963;11(6):633-639

[3] Shanks HE, Bickmore RW. Four dimensional electromagnetic radiators. Canadian Journal of Physics. 1959;37(3): 263-275

[4] Mandal SK, Mahanti GK, Ghatak R. Differential evolution algorithm for optimizing the conflicting parameters in time-modulated linear array antennas. Progress In Electromagnetics Research, PIER B. 2013;51:101-118

[5] Yang S, Gan YB, Tan PK. A new technique for power-pattern synthesis in time-modulated linear arrays. IEEE Antennas and Wireless Propagation Letters. 2003;2:285-287

[6] Fondevila J, Bregains JC, Ares F, et al. Optimizing uniformly excited linear arrays through time modulation. IEEE Antennas and Wireless Propagation Letters. 2004;3(1):298-301

[7] Poli L, Rocca P, Oliveri G, et al. Harmonic beamforming in timemodulated linear arrays. IEEE Transactions on Antennas and Propagation. 2011;59(7):2538-2545

[8] Mandal SK, Mahanti GK, Ghatak R. Synthesis of simultaneous multipleharmonic-patterns in time-modulated linear antenna arrays. Progress In Electromagnetics Research M. 2014;34: 135-142

[9] Li G, Yang S, Chen Y, Nie Z. A novel electronic beam steering technique in time modulated antenna arrays.
Progress in Electromagnetic Research PIER. 2009;97:391-405

[10] Song Q, Wang Y, Liu K, Zhang J, Wang Y. Beam steering for OAM beams using time-modulated circular arrays. Electronics Letters. 2018;54(17): 1017-1018

[11] He C et al. Direction finding by time-modulated linear array. IEEE Transactions on Antennas and Propagation. 2018;66(7):3642-3652

[12] Masoti D, Costanzo A, Del Prete M, Rizzoli V. Time-modulation of linear arrays for real-time reconfigurable wireless power transmission. IEEE Transactions on Microwave Theory and Techniques. 2016;64(2):331-342

[13] Rocca P, Yang F, Poli L, Yang S. Time-modulated array antennas Theory, techniques, and applications. Journal of Electromagnetic Waves and Applications. 2019;33(22):1503-1531

[14] Bregains JC, Fondevila-Gomez J, Franceschett G, et al. Signal radiation and power losses of time-modulated arrays. IEEE Transactions on Antennas and Propagation. 2008;56(6):1799-1804

[15] Mandal SK, Mandal R, Mahanti GK, Ghatak R. Characteristics of sideband radiation of uniformly excited time modulated antenna arrays (TMAA) through uniform variation of 'switchon' time. In: IEEE 2011 India Conference (INDICON 2011). Hyderabad, India; 2011. pp. 1-3

[16] Haupt RL. An introduction to genetic algorithms for electromagnetics. IEEE AP Magazine. 1995;2:7-15

[17] Mandal SK, Ghatak R, Mahanti GK. Influence on side band radiation of uniformly excited TMAA during reduction of SLL of the main beam. 
In: IEEE Indian Antenna Week (IAW), Kolkata, W.B. 2011. pp. 1-4

[18] Poli L, Rocca P, Manica L, Massa A. Pattern synthesis in time-modulated linear arrays through pulse shifting. IET Microwaves, Antennas and Propagation. 2010;4(9):1157-1164

[19] Mandal SK, Mahanti GK, Rowdra G. Optimizing time delay of time modulated linear Array elements to reduce the side band radiation level. In: International Conference on Signal Processing, Communications and Computing (ICSPCC). Hong Kong; 2012. pp. 556-559

[20] Yang S, Gan YB, Qing A, Tan PK. Design of a uniform amplitude time modulated linear array with optimized time sequences. IEEE Transactions on Antennas and Propagation. 2005;53(7): 2337-2339

[21] Zhu Q, Yang S, Zheng L, Nie Z. Design of a low sidelobe time modulated linear array with uniform amplitude and sub-sectional optimized time steps. IEEE Transactions on Antennas and Propagation. 2012;60(9):4436-4439

[22] Mandal SK, Ghatak R, Mahanti GK. Design of a time-modulator to synthesize different patterns in timemodulated antenna arrays. Journal of Electromagnetic Waves and Application. 2014;28(09):1118-1130

[23] He C, Yu H, Liang X, Geng J, Jin R. Sideband radiation level suppression in time-modulated array by nonuniform period modulation. IEEE Antennas and Wireless Propagation Letters. 2015;14: 606-609

[24] Mandal S, Mandal SK. Harmonic power losses in time modulated arrays with non-uniform period modulation. AEU - International Journal of Electronics and Communications. 2019; 108:45-52
[25] Kanbaz I, Yesilyurt U, Aksoy E. A study on harmonic power calculation for nonuniform period linear time modulated arrays. IEEE Antennas and Wireless Propagation Letters. 2018; 17(12):2369-2373

[26] Guo J, Yang S, Chen Y, Rocca P, $\mathrm{Hu}$ J, Massa A. Efficient sideband suppression in 4-D antenna arrays through multiple time modulation frequencies. IEEE Transactions on Antennas and Propagation. 2017;65(12): 7063-7072

[27] Durr M, Trastoy A, Ares F. Multiple-pattern linear antenna arrays with single prefixed amplitude distributions: Modified WoodwardLawson synthesis. Electronics Letters. 2000;36(16):1345-1346

[28] Chakraborty A, Das BN, Sanyal GS. Beam shaping using nonlinear phase distribution in a uniformly spaced array. IEEE Transactions on Antennas and Propagation. 1982;30: 1031-1034

[29] Bucci OM, Mazzarella G, Panariello G. Reconfigurable arrays by phase-only control. IEEE Transactions on Antennas and Propagation. 1991; 39(7):919-925

[30] Yang S, Gan YB, Qing A, et al. Design of uniform amplitude time modulated linear array with optimized time sequences. IEEE Transactions on Antennas and Propagation. 2005;53(7): 2337-2339

[31] Yang S, Gan YB, Qing A. Sideband suppression in time-modulated linear arrays by the differential evolution algorithm. IEEE Antennas and Wireless Propagation Letters. 2002;1:173-175

[32] Mandal SK, Ghatak R, Mahanti GK. Design of digitally-controlled multiple pattern time-modulated antenna arrays with phase-only difference. Annals of 
Telecommunications - annales des telecommunications. 2015;70(1-2):29-35

[33] Fondevila J, Brégains JC, Ares F, et al. Application of time-modulation in the synthesis of sum and difference patterns by using linear arrays. Microwave and Optical Technology Letters. 2006;48:829-832

[34] Mandal SK, Ghatak R, Mahanti GK. Minimization of side lobe level and side band radiation of a uniformly excited time modulated linear antenna array by using artificial Bee Colony algorithm. In: Industrial Electronics and Applications (ISIEA 2011). Langkawi, Malaysia; 2011. pp. $247-250$

[35] Pal S, Das S, Basak A. Design of time-modulated linear arrays with a multi-objective optimization approach. Progress In Electromagnetics Research, PIER. 2010;23:83-107

[36] Chen Y, Yang S, Nie Z. Improving conflicting specifications of timemodulated antenna arrays by using a multiobjective evolutionary algorithm. International Journal of Numerical Modelling. 2012;25(3):205-215

[37] Karaboga D, Basturk B. A powerful and efficient algorithm for numerical function optimization: Artificial bee colony $(\mathrm{ABC})$ algorithm. Journal of Global Optimization. 2007;39(3): 459-471

[38] Akay B, Karaboga D. Parameter tuning for the artificial bee Colony algorithm. In: Proceedings of ICCCI 2009, LNCS. Vol. 5796. Springer-Verlog. pp. 608-619

[39] Aksoy E, Afacan E. Calculation of sideband power radiation in timemodulated arrays with asymmetrically positioned pulses. IEEE Antennas and Wireless Propagation Letters. 2012;11: 133-136

[40] Poli L, Rocca P, Manica L, Massa A. Handling sideband radiations in time-modulated arrays through particle swarm optimization. IEEE Transactions on Antennas and Propagation. 2010; 58(4):1408-1411

[41] Tong Y, Tennant A. Sideband level suppression in time-modulated linear arrays using modified switching sequences and fixed bandwidth elements. Electronics Letters. 2012; 48(1):10-11

[42] Zhu Q, Yang S, Yao R, Nie Z. Gain improvement in time-modulated linear arrays using SPDT switches. IEEE Antennas and Wireless Propagation Letters. 2012;11:994-997 


\title{
Convex Optimization and Array Orientation Diversity-Based Sparse Array Beampattern Synthesis
}

\author{
Hui Chen and Qun Wan
}

\begin{abstract}
The sparse array pattern synthesis (APS) has many important implications in some special situations where the weights, size, and cost of antennas are limited. In this chapter, the APS with a minimum number of elements problem is investigated from the perspective of sparseness constrained optimization. Firstly, to reduce the number of antenna elements in the array, the APS problem is formulated as sparseness constrained optimization problem under compressive sensing (CS) framework and solved by using the reweighted L1-norm minimization algorithm. Besides, to address left-right radiation pattern ambiguity problem, the proposed algorithm exploits the array orientation diversity in the sparsity constraint framework. Simulation results demonstrate the proposed method's validity of achieving the desired radiation beampattern with the minimum number of antenna elements.
\end{abstract}

Keywords: array beampattern synthesis, compressive sensing, array orientation diversity, convex optimization

\section{Introduction}

The objective of array pattern synthesis (APS) is to find the excitation of an array to produce a radiation beampattern which is close to the desired one. DolphChebyshev method $[1,2]$ can be used to design an optimal pattern with the minimum sidelobe level and desired mainlobe width for a uniform linear array (ULA) with isotropic elements. While it is more difficult to solve the APS problem for an array of arbitrary geometric structures.

For nonuniformly spaced arbitrary arrays, there are several algorithms [3-6] that have been proposed to synthesize beampatterns. The design of thinned narrowbeam arrays has been well proposed in [3], which first fix element locations by eliminating the elements pair by pair according to the smallest possible sidelobe on the given interval and then optimize the weights via linear programming. For APS problem, which can also be formulated as a quadratic programming problem $[4,5]$, the objective function is to minimize the squared errors between the synthesized pattern and the desired pattern. Besides, additional linear constraints [4] or weighting functions [7] are also added to the quadratic objective function to minimize the peaks of the synthesis error. The challenge to weighting functions in the 
quadratic programming is that it has to be adjusted in an ad hoc manner. Besides, an inverse matrix has to be computed at each iteration for updating the weighting functions, which will result in high computation requirements, especially for large size of the array. The author of [8] proposed a recursive least squares method to solve the problem. Another kind of evolutionary algorithm, such as simulated annealing [9], particle swarm optimization [10], and genetic algorithm [11-13], has also been used for APS problem optimization.

Recently, second-order cone programming (SOCP) and semi-definite programming (SDP), as convex optimization techniques [14, 15], have been proposed to solve the APS problem readily by using SOCP solver and SDP solver, respectively. While a general nonuniform APS problem cannot be directly formulated as a convex problem. An iterative procedure [15] was proposed to optimize the array pattern by solving an SDP problem at each iteration. All the abovementioned approaches to design an optimal nonuniform array are to construct an objective function of minimizing the synthesis error or peak error. When the positions of elements are given, the nonuniformly spaced arrays can be optimized using convex programming like that for uniformly spaced arrays. While it is impossible to solve the APS problem by complex programming if the positions of the array elements are unknown. In addition, to solve the problem of occupying more elements to obtain the desired beampattern, the authors in [16] proposed a matrix pencil-based noniterative synthesis algorithm, which can efficiently save the number of elements in a very short computation time. Zhang et al. [17] formulated the APS problem as a sparseness constrained optimization problem and solved the problem by using Bayesian compressive sensing (BCS) inversion algorithm; the authors in [18] proposed an approach for APS of linear sparse arrays, and then the multitask BCS has been used to design 2D sparse synthesis problem [19], sparse conformal array synthesis problem [20-22], and another CS-based sparse array synthesis problem [23-26].

In this chapter, we proposed an array pattern synthesis algorithm [27] by using reweighted $l_{1}$-norm minimization [28] and convex optimization [29]. Then we extended our work to a new version [30] by using reweighted $l_{1}$-norm minimization and array orientation diversity. Merits of the algorithm include the following:

(1) it does not need a thorough search in the multidimensional parameter space, and

(2) it can achieve the same array performance with fewer antenna elements when the array size is given and thus reduces the array cost significantly. Regarding the notation of this chapter, $(\cdot)^{T}$ represents the transpose operation of a vector or matrix, $|\cdot|$ denotes the absolute value operator, and $\|\cdot\|_{1}$ and $\|\cdot\|_{\infty}$ represent the $l_{1}$-norm and $l_{\infty}$-norm of a vector or matrix, respectively. And $\lceil x\rceil$ denotes the smallest integer not less than $x$, and $\operatorname{diag}(x)$ means the diagonal matrix with the main diagonal elements equaled to the vector $x$.

\section{Nonuniform array pattern synthesis using reweighted $l_{1}$-norm minimization}

\subsection{Problem formulation}

Consider a narrowband linear array with $M$ isotropic antennas located at $x_{1}, \ldots, x_{M} \in R^{2}$. Assume that a harmonic plane wave with wavelength $\lambda$ propagates across the array with incident direction $\theta$. The $M$ signal outputs $s_{i}$ are converted to the baseband, weighted by the weights $w_{i}$, and summed. Then the array response can represented as 


$$
G(\theta)=\sum_{i=1}^{M} w_{i} \exp \left(j 2 \pi x_{i} \sin \theta / \lambda\right)=\boldsymbol{w}^{T} \boldsymbol{a}(\theta)
$$

where $\phi_{i}=2 \pi x_{i} \sin \theta / \lambda$ is the phase delay due to propagation, complex weight vector $\boldsymbol{w}=\left[w_{1}, \ldots, w_{M}\right]^{T} \in C^{M}$, and the steering vector $\boldsymbol{a}(\theta)$.

Let $G_{d}(\theta)$ be the desired array response at the direction $\theta$. The APS problem is to find the complex weight vector $w$ such that $G(\theta)=G_{d}(\theta)$ for all $\theta \in\left[-90^{\circ}, 90^{\circ}\right]$. For the array described above, how well $G(\theta)$ approximates $G_{d}(\theta)$ can be measured by using the peak error across $\theta$, i.e.,

$$
\min _{w} \max _{\theta \in \Theta}\left|G(\theta)-G_{d}(\theta)\right|
$$

where $\Theta \in\left[-90^{\circ}, 90^{\circ}\right]$ is a dense set of arrival angles that we are of interest. The goal of the proposed algorithm is to find both optimal antenna locations and corresponding weights that approach the desired array pattern as well as possible.

\subsection{The proposed algorithm}

The APS problem can be formulated as a following estimation problem:

$$
\boldsymbol{w}^{T} \boldsymbol{a}(\theta)=G(\theta), \forall \theta \in \Theta
$$

We try to find $w$ in Eq. (3) such that Eq. (2) is satisfied.

The new solution of Eq. (3) can be summarized as follows:

\subsubsection{Creating a virtual array}

For a given array size, to obtain more elements than those of a conventional array with $\lambda / 2$ inter-element spacing, we first create a dense uniformly spaced linear array with much smaller inter-element spacing than conventional array and initialize a weight matrix $Q$ as an identity matrix to create a more sparse array in subsequent processing.

\subsubsection{Finding the sparse weight vector}

The specified synthesized pattern $G(\theta)$ is produced by a weight vector. The weight vector can be obtained by solving the following weighted $l_{1}$-norm minimization convex problem Eq. (4), which is subject to minimizing the peak of the error between the synthesized pattern $G(\theta)$ and the desired pattern $G_{d}(\theta)$ :

$$
\begin{aligned}
& \text { Minimize }\|\boldsymbol{Q} w\|_{1} \\
& \text { Subject to }\left\|G(\theta)-G_{d}(\theta)\right\|_{\infty} \leq \varepsilon, \forall \theta \in\left[-90^{\circ}, 90^{\circ}\right]
\end{aligned}
$$

where $\varepsilon$ is the fitting error between the synthesized pattern and desired pattern. Minimizing $\|Q w\|_{1}$ makes the vector $Q w$ sparse, which is useful to create a nonuniformly spaced array. According to the situation that some weights of the original weight vector $\boldsymbol{w}=\left[w_{1}, w_{2}, \ldots\right]^{T}$ from Eq. (4) are very small, they can be deleted without significantly decreasing the array performance. So a sparse weight vector can be obtained by retuning the small value elements of the original weight vector, that is, the $w_{i}$ will be retained if $\left|w_{i}\right| /\|\boldsymbol{w}\|_{\infty}>\eta(i=1,2, \ldots)$, otherwise $w_{i}=0$. The $\eta$ is a designed threshold whose value should make a trade-off between 
APS performance and convergence rate. Because more elements of the original weight vector will be pruned if the threshold value $\eta$ is increased, which make us probably cannot find the optimal array element positions of the array, correspondingly the array synthesis performance is not optimal for a given array element number. Conversely, if the threshold value is decreased, less elements of the original weight vector will be pruned in each iteration, which increases the algorithm complexity. So we should make a good balance between APS performance and convergence rate when setting the value of $\eta$.

\subsubsection{Updating the weight matrix}

After obtaining the original weight vector $\boldsymbol{w}=\left[w_{1}, w_{2}, \ldots\right]^{T}$, the weight matrix $\boldsymbol{Q}$ is updated as $\boldsymbol{Q}=\operatorname{diag}\left(\left[\left(\left|w_{1}\right|+\delta\right)^{-p},\left(\left|w_{2}\right|+\delta\right)^{-p}, \ldots\right]\right)$ (usually, $p$ is an integer greater than 1; it was demonstrated experimentally that $p=2$ is a better choice for our APS problem). To ensure that the weight matrix is effectively updated when a zero-valued component in $w$, we introduce a parameter $\delta>0$. It is empirically demonstrated that $\delta$ should be set slightly smaller than the expected nonzero magnitudes of $\boldsymbol{w}$.

\subsubsection{Forming the nonuniform array}

The sparse weight vector $\boldsymbol{w}_{s}$ is obtained by pruning the original weight vector, and then the antenna elements corresponding to nonzero-valued indices of $\boldsymbol{w}_{s}$ are retained to form a nonuniform array with fewer elements.

The above steps (A, B, C) are repeated until the final synthesized array performance is satisfactory or the specified maximum number of iterations is attained.

\subsubsection{Optimizing the sparse weight vector}

After obtaining the array antenna positions by steps (B, C, D), the optimal weight vector $\boldsymbol{w}_{\text {opt }}$ is further obtained by solving following convex optimization problem, which is to improve the performance of the array beampattern synthesized by the sparse weight vector:

$$
\begin{aligned}
& \text { Find } \boldsymbol{w}_{\text {opt }} \\
& \text { Minimize }\left\|G(\theta)-G_{d}(\theta)\right\|_{\infty}, \theta \in\left[-90^{\circ}, 90^{\circ}\right]
\end{aligned}
$$

\subsection{Computer simulation and discussions}

Given the array aperture, the objective is to design an array with the beampattern as shown in Figure 1, where region $|\theta| \leq \theta_{s}$ corresponds to the mainlobe and region $|\theta| \geq \theta_{s}$ corresponds to the sidelobe. We set $\theta_{s}=2.5^{\circ}$, and the angle grid of the interval $\left[-90^{\circ}, 90^{\circ}\right]$ is $1^{\circ}$. We design a virtual ULA with the array aperture of $25.5 \lambda$ having a uniform inter-element spacing of $\lambda / 8$.

The beampattern of Figure 2 is obtained by using our approach for a 19-element array, and the optimal beampattern exhibits the maximum sidelobe of $-15.46 \mathrm{~dB}$. The optimal antenna positions and the corresponding weights are displayed in Table 1. The designs proposed in $[3,17]$ describe a 25 -element and a 29-element non-ULA with the approximate desired array pattern shown in Figure 2, respectively. The 25-element array beampattern described in [3] by eliminating the elements pair by pair has a maximum sidelobe $-13.75 \mathrm{~dB}$, while the maximum sidelobe of 29-element array beampattern obtained by the BCS algorithm [17] is $-13.165 \mathrm{~dB}$. 
Convex Optimization and Array Orientation Diversity-Based Sparse Array Beampattern Synthesis DOI: http://dx.doi.org/10.5772/intechopen.88881

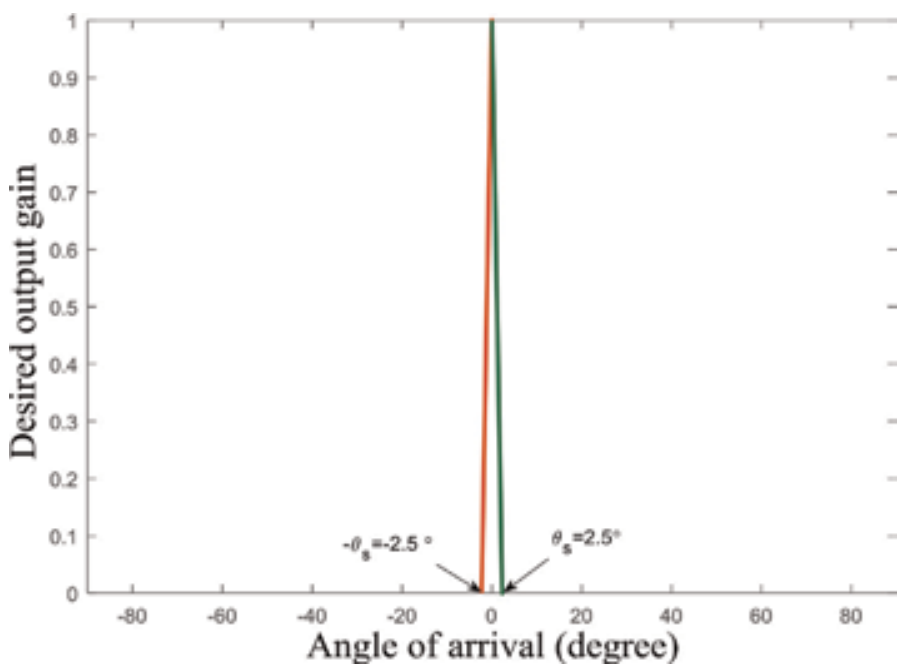

Figure 1.

The desired beampattern.

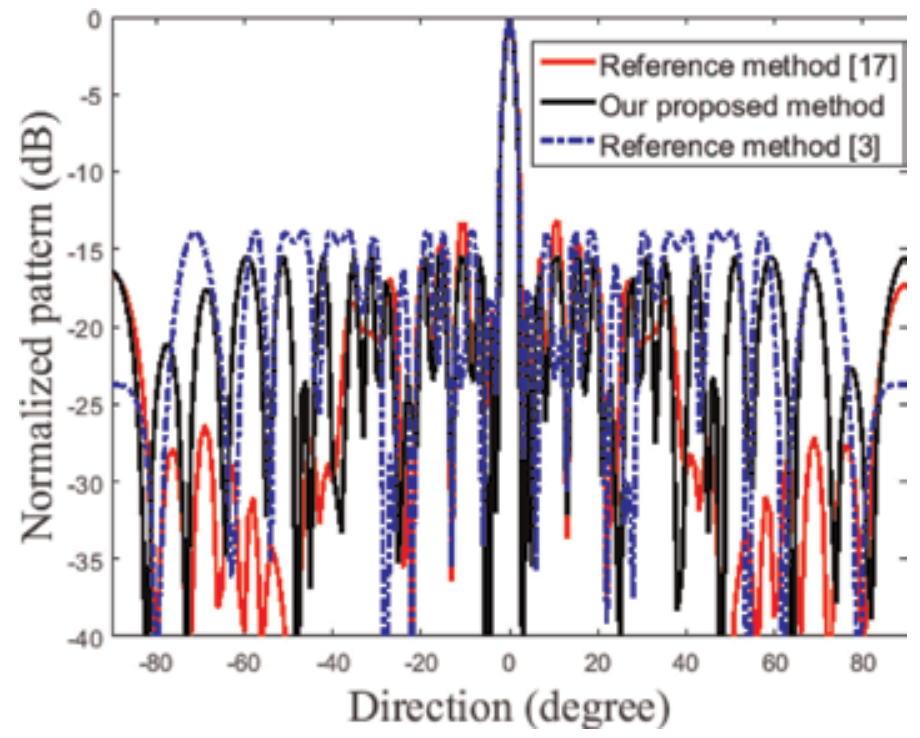

Figure 2.

A "25-element array beampattern obtained by [3] and a 29-element array beampattern obtained by [17]" vs. "our 19-element array beampattern."

\begin{tabular}{lccccc}
\hline Element indices & Position $(\lambda)$ & Weight value & Element indices & Position $(\lambda)$ & Weight value \\
\hline 1,19 & \pm 10.500 & 0.2289 & 6,14 & \pm 3.875 & 0.2677 \\
\hline 2,18 & \pm 8.625 & 0.2583 & 7,13 & \pm 2.875 & 0.2195 \\
\hline 3,17 & \pm 7.6250 & 0.2207 & 8,12 & \pm 1.875 & 0.1813 \\
\hline 4,16 & \pm 6.750 & 0.2904 & 9,11 & \pm 1.000 & 0.2347 \\
\hline 5,15 & \pm 4.750 & 0.1567 & 10 & 0 & 0.2427 \\
\hline
\end{tabular}

Table 1.

Our element positions and weights in a 19-element antenna array. 
The antenna positions and the corresponding weights of the two methods in $[3,17]$ are listed in Tables 2 and 3, respectively. Compared with the method in [3], we can see from Table 1 and Figure 2 that our proposed algorithm saves six elements without reducing the array performance and the our minimum inter-element spacing of the non-ULA is $0.375 \lambda$ larger than that of the method of eliminating the elements pair by pair [3]. Compared with the reference method in [17], our proposed method offers an economization of 10 elements as well as $2.3 \mathrm{~dB}$ performance improvement, and the minimum inter-element spacing of the sparse array designed by our approach is $0.75 \lambda$ larger than that of the reference array [17]. We also emphasize that the reference array [17] has $4.5 \lambda$ larger array aperture than that of our array.

The proposed APS algorithm based on convex optimization and reweighted $l_{1}$-norm minimization is proven to be effective in reducing array elements, suppressing the sidelobe, and reducing the aperture. This simple and effective design method can be extended to solving the $2 \mathrm{D}$ array synthesis problem.

\begin{tabular}{lccccc}
\hline Element indices & Position $(\lambda)$ & Weight value & Element indices & Position $(\lambda)$ & Weight value \\
\hline 1,25 & \pm 12.0 & 0.2100 & 8,18 & \pm 4.5 & 0.1924 \\
\hline 2,24 & \pm 8.5 & 0.2605 & 9,17 & \pm 3.5 & 0.2296 \\
\hline 3,23 & \pm 8.0 & 0.2276 & 10,16 & \pm 2.0 & 0.2282 \\
\hline 4,22 & \pm 7.5 & 0.2554 & 11,15 & \pm 1.5 & 0.0876 \\
\hline 5,21 & \pm 7.0 & 0.2103 & 12,14 & \pm 0.5 & 0.1143 \\
\hline 6,20 & \pm 6.0 & 0.200 & 13 & 0 & 0.2084 \\
\hline 7,19 & \pm 5.0 & 0.2037 & & & \\
\hline
\end{tabular}

Table 2.

Element positions and weights obtained in a 25-element array [3].

\begin{tabular}{lcc}
\hline Element indices & Position $(\lambda)$ & Weight value \\
\hline 1,2 & $-1.375,-0.500$ & $0.0876,0.1178$ \\
\hline 3,4 & $0.375,0.500$ & $0.0532,0.1025$ \\
\hline 5,6 & $1.250,1.375$ & $0.0497,0.1844$ \\
\hline 7,8 & $2.125,4.375$ & $0.1895,0.1086$ \\
\hline 9,10 & $4.500,5.250$ & $0.0778,0.2679$ \\
\hline 11,12 & $6.125,7.000$ & $0.2440,0.1098$ \\
\hline 13,14 & $7.125,8.125$ & $0.0643,0.2400$ \\
\hline 15,16 & $8.875,10.125$ & $0.1953,0.2249$ \\
\hline 17,18 & $11.000,11.750$ & $0.2297,0.0720$ \\
\hline 19,20 & $12.000,12.750$ & $0.1480,0.1810$ \\
\hline 21,22 & $13.750,13.875$ & $0.0761,0.0554$ \\
\hline 23,24 & $14.875,15.750$ & $0.0840,0.1833$ \\
\hline 25,26 & $16.625,16.750$ & $0.1860,0.0516$ \\
\hline 27,28 & $17.375,19.625$ & $0.1625,0.0745$ \\
\hline 29 & 24.125 & 0.0317 \\
\hline
\end{tabular}

Table 3.

Element positions and weights obtained by the BCS inversion algorithm [17]. 


\section{Beampattern synthesis using reweighted $l_{1}$-norm minimization and array orientation diversity}

To address left-right radiation pattern ambiguity problem, we allow exploitation of the array orientation diversity in the CS framework.

\subsection{Problem formulation}

We assume that transmit signals and the array are coplanar, so the antenna array synthesis problem can be described as follows:

$$
\min (D M) \quad \text { s.t. }\left\{\min _{\substack{\left\{R_{\alpha i}, d_{\alpha i}\right\}_{\alpha=1, \ldots . D} \\ i=1, \ldots, M}}\left\|F_{d}(\theta)-F(\theta)\right\|_{l_{2}}\right\} \leq \xi
$$

where $F(\theta)=\sum_{\alpha=1}^{D} \sum_{i}^{M} R_{\alpha i} e^{j k d_{\alpha i} \cos \left(\theta-\theta_{\alpha}\right)}, F_{d}(\theta)$ is the desired radiation pattern, $M$ is the number of identical antenna elements in each linear array, $R_{\alpha i}$ is the excitation coefficient of the $i$ th element located at $d_{\alpha i}$ in the $\alpha$ th array, $k$ is the wavenumber in the free space, and $D$ array orientations $\theta_{\alpha}(\alpha=1, \ldots, D)$. The objective of the problem is to synthesize the desired radiation pattern $F_{d}(\theta)$ with the minimum number of elements under a small tolerance error $\xi$. For one linear array at orientation $\theta_{\alpha}$ to the incident plane wave from the bearing $\theta$, the array factor is given by

$$
F_{\alpha}(\theta)=\sum_{i}^{M} R_{\alpha i} e^{j k d_{\alpha i} \cos \left(\theta-\theta_{\alpha}\right)}
$$

Suppose that all the antenna elements in each array orientation $\theta_{\alpha}(\alpha=1, \ldots, D)$ are symmetrically distributed within a range of $-d_{s}$ to $d_{s}$ along the array orientation $\theta_{\alpha}$, respectively, the combination pattern of all the linear orientation arrays can be written as

$$
F(\theta)=\sum_{\alpha=1}^{D} F_{\alpha}(\theta)
$$

In order to solve Eqs. (7) and (8), we can assume that all the antenna elements are equally spaced from $-d_{s}$ to $d_{s}$ with a small inter-element spacing $\Delta d$. Although it is supposed that there is one element at each position, not each antenna element is necessarily radiating waves or excited with current. All the antenna elements can be in two states: "on" states (when the element is in the supposed position or has an excitation) and "off" state (when there is no element in the supposed position or without an excitation). Through discretization, Eq. (8) can be written in a matrix form:

$$
[F(\theta)]_{h \times 1}=[H]_{h \times n}[r]_{n \times 1}
$$

where $\mathrm{h}$ is the number of sampled antenna radiation pattern, $n=D\left\lceil\frac{2 d_{s}}{\Delta d}\right\rceil$, the sensing radiation pattern at different angles is contained in vector $\mathbf{F}=\left[F\left(\theta_{1}\right) F\left(\theta_{2}\right) \cdots F\left(\theta_{h}\right)\right]^{T}$, overcomplete dictionary $\mathbf{H}$ is an $h \times n$ matrix whose $(i, l)$ th element is $\mathbf{H}_{i l}=e^{j k d_{\alpha i} \cos \left(\theta_{l}-\theta_{\alpha}\right)}, l \in\left((\alpha-1) \frac{n}{D}+1, \frac{n}{D} \alpha\right)$ for $\alpha \in\{1, \ldots, D\}$, and $h \ll n . r$ is an excitation vector, $R_{\alpha i}=0$ means the antenna in the $l$ th position of the 
$\alpha$ th array is absent from the supposed position, and the solution of sparse excitation vector $r$ can be casted as the following convex optimization problem:

$$
\begin{aligned}
& \min \|\boldsymbol{r}\|_{1} \\
& \text { subject to }\|\mathbf{F}-\mathbf{H} \boldsymbol{r}\|_{\infty} \leq \xi
\end{aligned}
$$

In Eq. (10) the smallest number of nonzero elements in the excitation vector $r$ can be obtained readily by using existing software package, such as CVX [31].

\subsection{The proposed algorithm}

In this subsection, the new solution of Eq. (6) can be summarized as follows:

\subsubsection{Initializing a virtual array and a weight matrix}

To place more antenna elements than those of a conventional array with the same array size, we first create $D$ virtual linear orientation arrays with much smaller interspacing $\lambda / 16$ (in general, the inter-element spacing of the conventional ULA is $\lambda / 2$ ). Using the reweighted $l_{1}$-norm minimization in the following step, we set a $D M \times D M$ dimension weight matrix $\mathbf{Q}$ as a unit matrix.

\subsubsection{Finding the sparse weight vector}

Let $F(\theta)$ be a synthesized beampattern by using a weight vector, and the weight vector can be obtained by solving the following weighted $l_{1}$-norm minimization convex problem which is to try to minimize the peak value of the error between the synthesized pattern and the desired pattern:

$$
\begin{aligned}
& \text { Minimize }\|Q \boldsymbol{w}\|_{1} \\
& \text { Subject to }\left\|F(\theta)-F_{d}(\theta)\right\|_{\infty} \leq \zeta, \forall \theta \in\left[-180^{\circ}, 180^{\circ}\right]
\end{aligned}
$$

where $\zeta$ is the fitting error between the synthesized pattern and the desired one. Minimizing $\|Q \boldsymbol{w}\|_{1}$ makes the vector $Q w$ sparse, which is useful to create $D$ nonuniformly spaced linear orientation arrays. Here, let the weight vector $\boldsymbol{w}=$ $\left[w_{1}, w_{2}, \ldots\right]^{T}$ obtained from Eq. (11) be the original weight vector for convenience. The weighted $l_{1}$-norm minimization will make some weights of the original weight vector be very small, so they can be adjusted to zero without significantly reducing the array performance. That is, if the absolute value of an element from the original weight vector is smaller than a threshold which is set according to the array performance requirement, the element will be assigned zero; otherwise, the element will be retained. Thus the sparse weight vector $w_{s}$ is obtained.

\subsubsection{Updating the weight matrix}

After obtaining the original weight vector $\boldsymbol{w}=\left[w_{1}, w_{2}, \ldots\right]^{T}$ from step (2), the weight matrix $\boldsymbol{Q}$ is updated according to $\boldsymbol{Q}=\operatorname{diag}\left(\left[\left(\left|w_{1}\right|+\delta\right)^{-p},\left(\left|w_{2}\right|+\delta\right)^{-p}, \ldots\right]\right)$ in each iteration; usually, $p$ is an integer greater than 1 , while it was demonstrated experimentally that $p=2$ is a better choice for our APS problem. To ensure regular update $Q$ especially for zero-valued components in $w$, we bring in the parameter $\delta>0$ which should be set slightly smaller than the expected nonzero magnitudes of $w$. Reweighted $l_{1}$ minimization can improve the signal reconstruction performance. 


\subsubsection{Creating the nonuniform arrays}

After obtaining the sparse weight vector $\boldsymbol{w}_{s}$ from step (4), the antenna elements corresponding to nonzero-valued indices of the sparse weight vector are retained to create $D$ sparse linear arrays with different orientations.

Repeat steps (2, 3, and 4) until the synthesized array beampattern performance is satisfactory or the specified maximum number of iterations or minimum antenna number is attained.

\subsubsection{Finding the optimal weight vector}

After optimizing the antenna element positions by the above steps, we introduce convex optimization to obtain the optimal weight vector which can further improve the performance of the array beampattern synthesized by the sparse weight vector:

$$
\begin{aligned}
& \text { Find } \boldsymbol{w}_{\text {opt }} \\
& \text { Minimize }\left\|F(\theta)-F_{d}(\theta)\right\|_{\infty}, \theta \in\left[-90^{\circ}, 90^{\circ}\right]
\end{aligned}
$$

The optimal sparse weight vector $\boldsymbol{w}_{\text {opt }}$ can be obtained from Eq. (12) readily.

\subsection{Computer simulations and discussion}

The objective is to design an array with the desired beampattern for given the array physical size, as shown in Figure 1, where region $|\theta| \leq \theta_{s}$ belongs to the mainlobe and region $|\theta| \geq \theta_{s}$ corresponds to the sidelobe. We set $\theta_{s}=2.3^{\circ}$, and the angle grid for the search area $\left[-180^{\circ}, 180^{\circ}\right]$ is $2^{\circ}$, that is, we take a "dense set" of $\left[-180^{\circ}, 180^{\circ}\right]$ with the angles sampled at $2^{\circ}$ from $-180^{\circ}$ to $180^{\circ}$ (Figure 3 ).

To show the performance of our beampattern synthesis, we will consider two cases, same element number array and same beampattern performance, since all formulated problems in Eqs. (6), (10), (11), and (12) are convex, so we adopt the optimization toolbox to solve the formulated problems.



Figure 3.

Desired beampattern. 


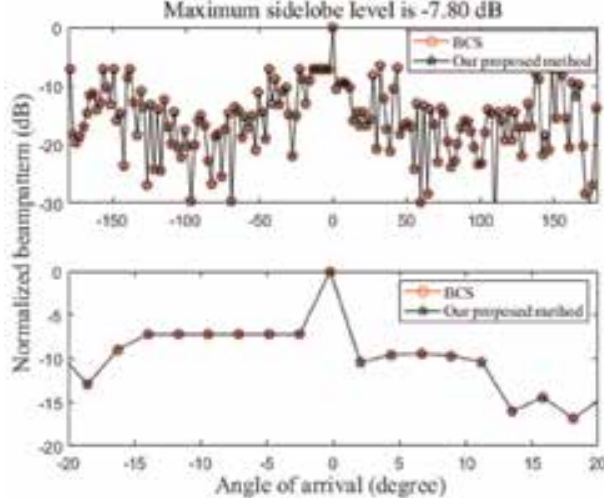

(a)

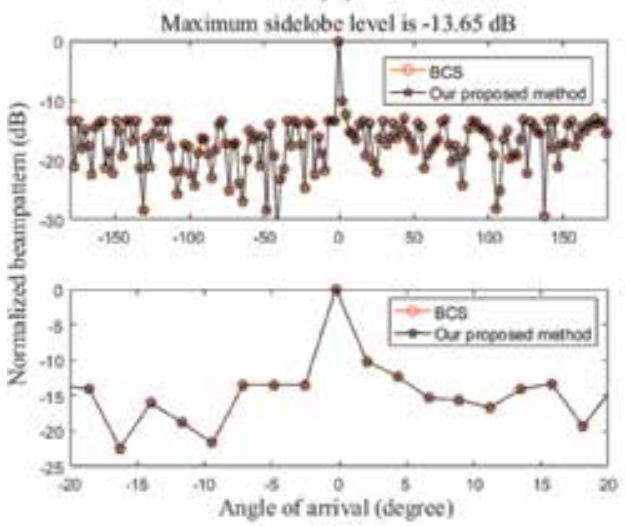

(c)

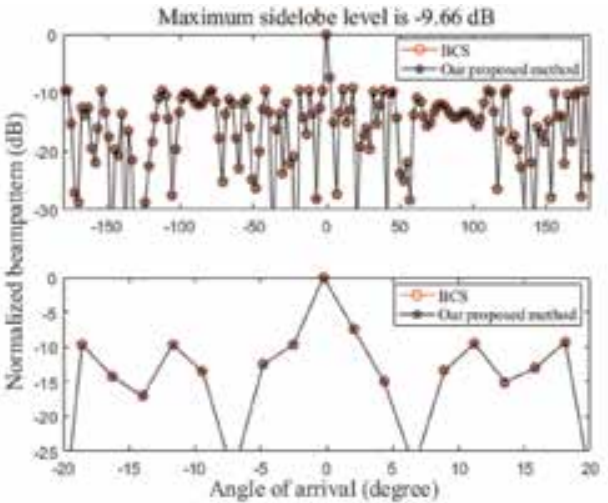

(b)

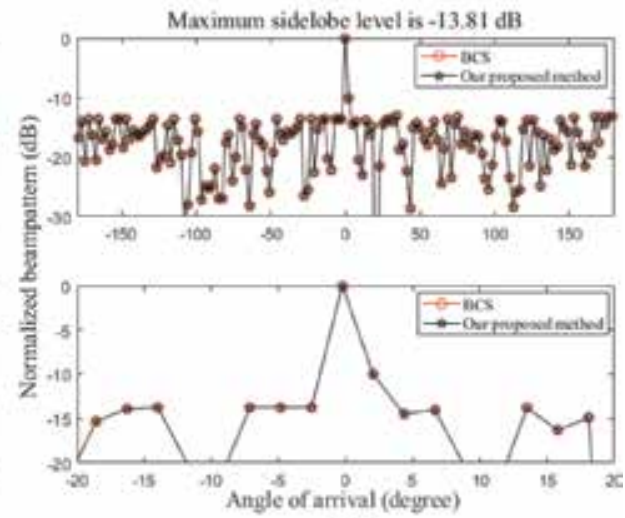

(d)

Figure 4.

A 19-element array performance obtained by BCS inversion algorithm [17] and our method with increasing array orientation diversity. (a) 1 array orientation, (b) 2 array orientations, (c) 3 array orientations, and (d) 4 array orientation.

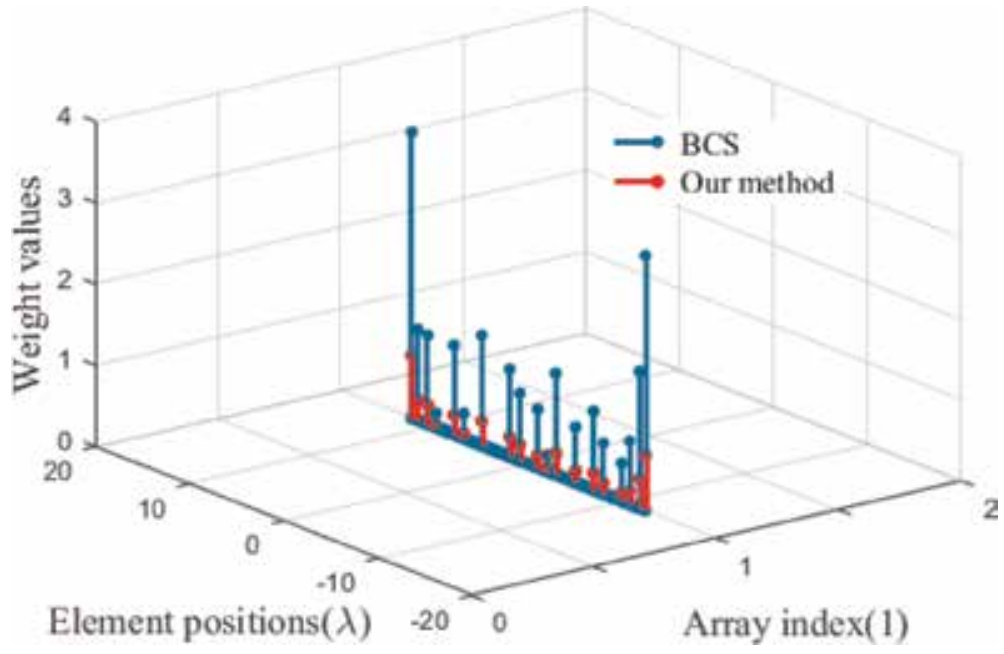

Figure 5 .

Element positions and excitation amplitudes in a 19-element one-array orientation. 


\subsubsection{Same element number array with array orientation diversity}

In this section, we analyzed the influence of the array orientation diversity on the beampattern synthesis by simulation results. We initialize four virtual ULAs (named Array 1, Array 2, Array 3, Array 4, with orientation $-10^{\circ}, 0^{\circ}, 10^{\circ}, 20^{\circ}$, respectively) with each subarray aperture of $25 \lambda$ owning a uniform interspacing $\lambda / 8$. Besides, we initialize $Q$ as a unit matrix and choose $\delta=10^{-4}$ and $p=2$ in our simulations. Figure 4 shows a 19-element beampattern synthesis performance in four cases with one-, two-, three-, and four-array orientations. From Figure 4, we can see that our proposed method and BCS algorithm can improve performance with increasing array orientation diversity (from 1 to 4 ); the optimal antenna positions and the corresponding excitation amplitudes of the four cases are displayed in Figures 5-8, respectively. Note that for the four cases of Figures 5-8, the required normalized radiated energies of BCS approach [17] are correspondingly bigger than that of our proposed method.

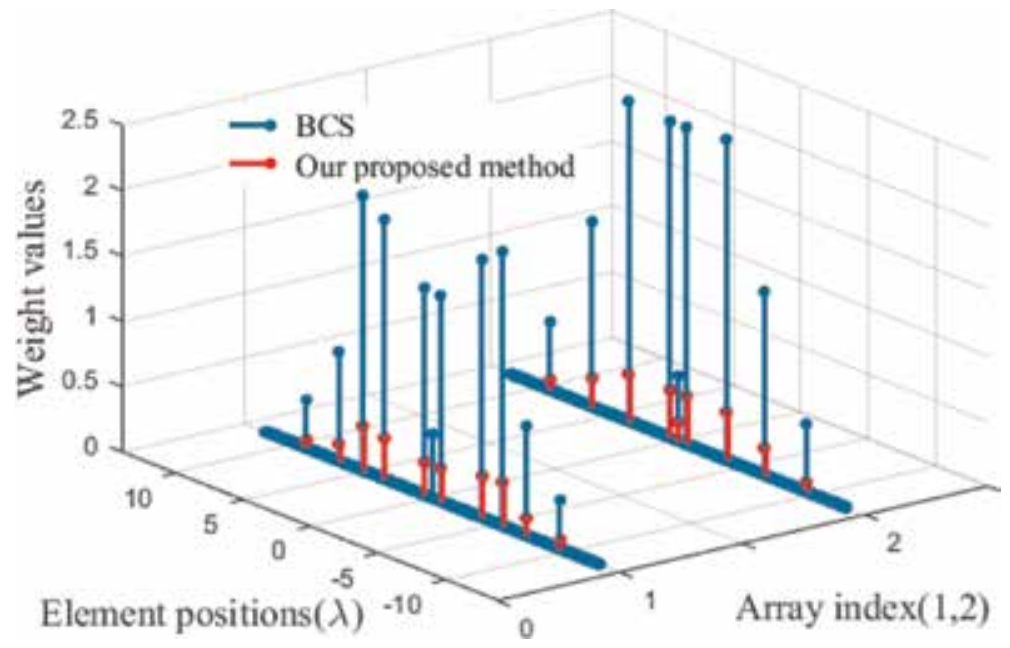

Figure 6.

Element positions and excitation amplitudes in a 19-element two-array orientation.

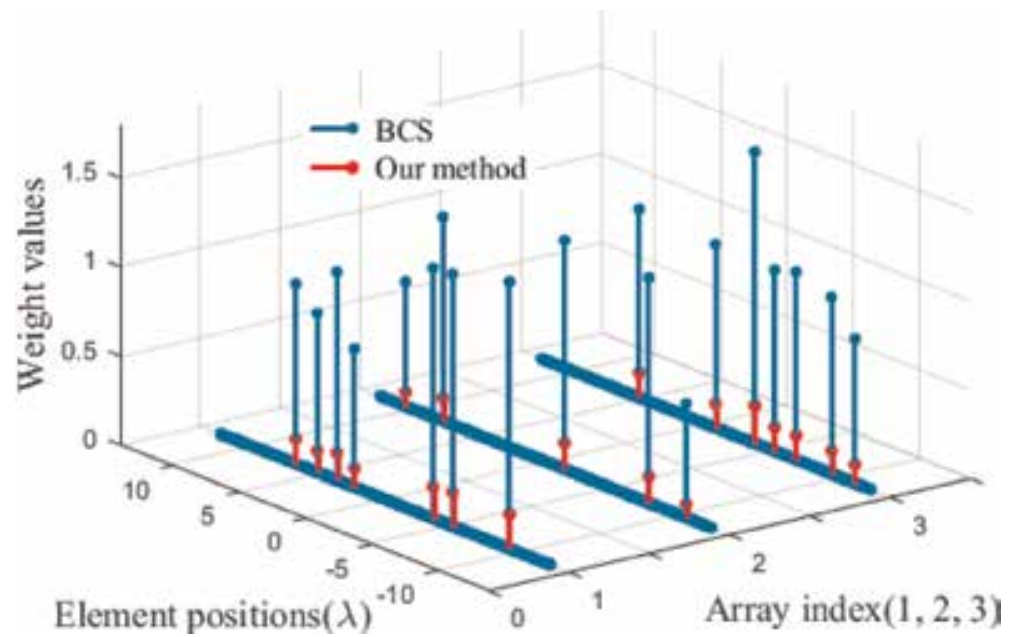

Figure 7.

Element positions and excitation amplitudes in a 19-element three-array orientation. 


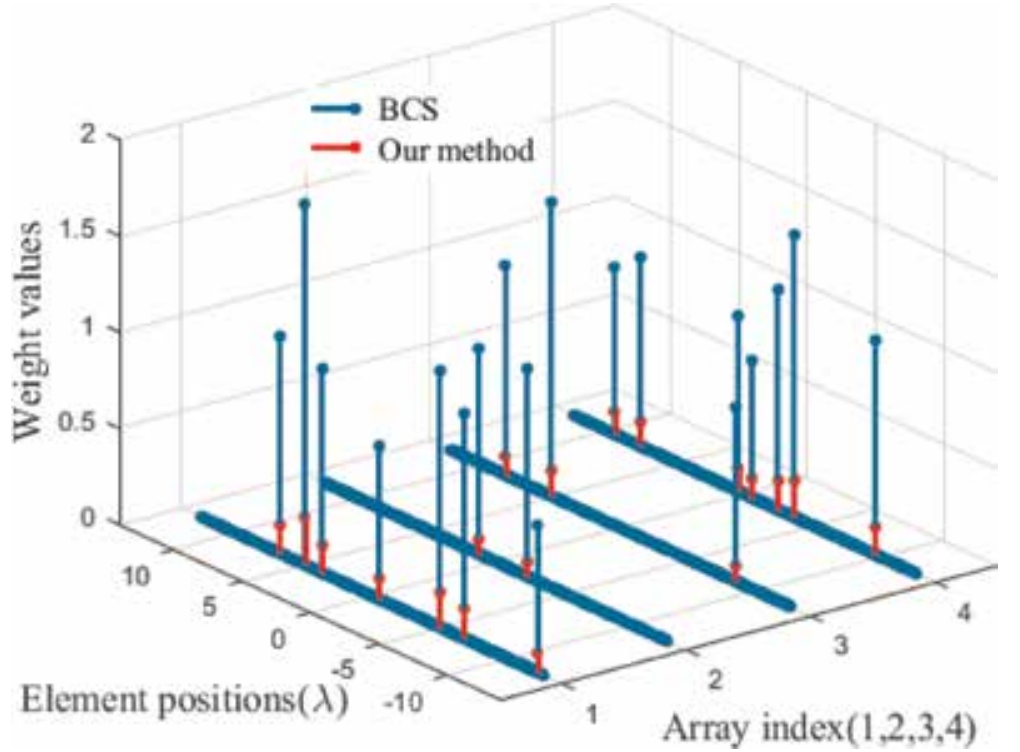

Figure 8.

Element positions and excitation amplitudes in a 19-element four-array orientation antenna.

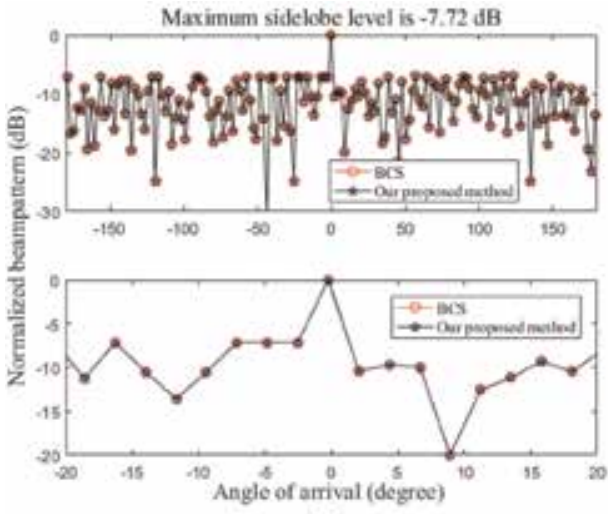

(a)

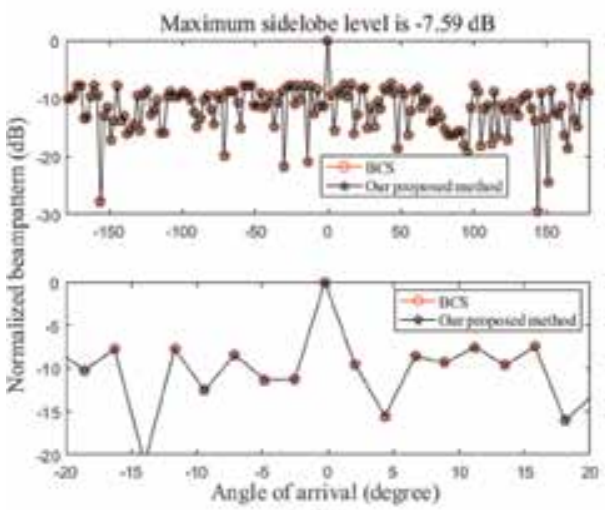

(c)

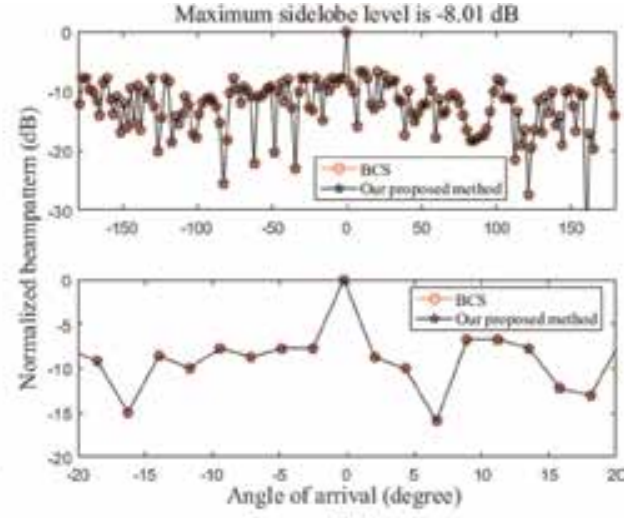

(b)

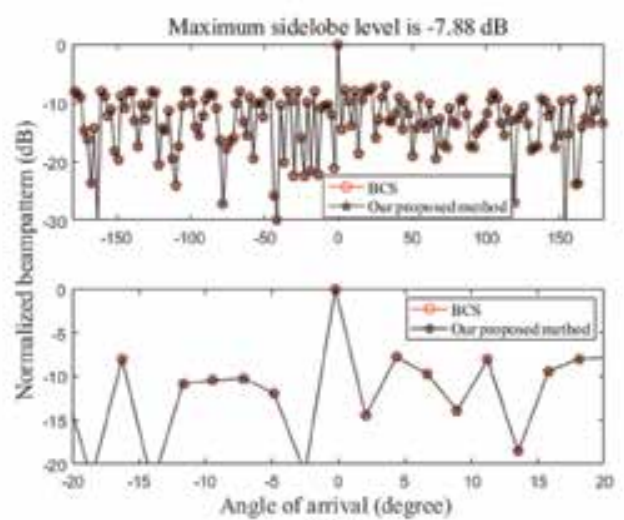

(d)

Figure 9.

Optimal beampattern of different element number array by using "BCS inversion algorithm [17]" vs. "our method." (a) 1 array orientation, (b) 2 array orientations, (c) 3 array orientations, and (d) 4 array orientations. 


\subsubsection{Approximate beampattern performance with array orientation diversity}

To demonstrate another advantage of array orientation diversity, we examine the beampattern synthesis of an 18-element array, 11-element array, 10-element array, and 10-element array correspondingly with one orientation, two orientations, three orientations, and four orientations using BCS algorithm and our method, respectively. The optimal beampatterns exhibit maximal sidelobes of $-7.72,-8.01,-7.59$, and $-7.88 \mathrm{~dB}$, respectively, which are shown in Figure 9.

Figures 10-13 provide all the corresponding antenna positions and excitation amplitudes for all the four cases mentioned above. Obviously, given the array size, using orientation diversity can economize seven (or eight) elements without reducing the array performance. But more diversity is not always better enough, as shown in Figures 11-13. Besides, the excitation amplitudes in Figures 10-13 show that our proposed method needs less radiation energy for all four cases.

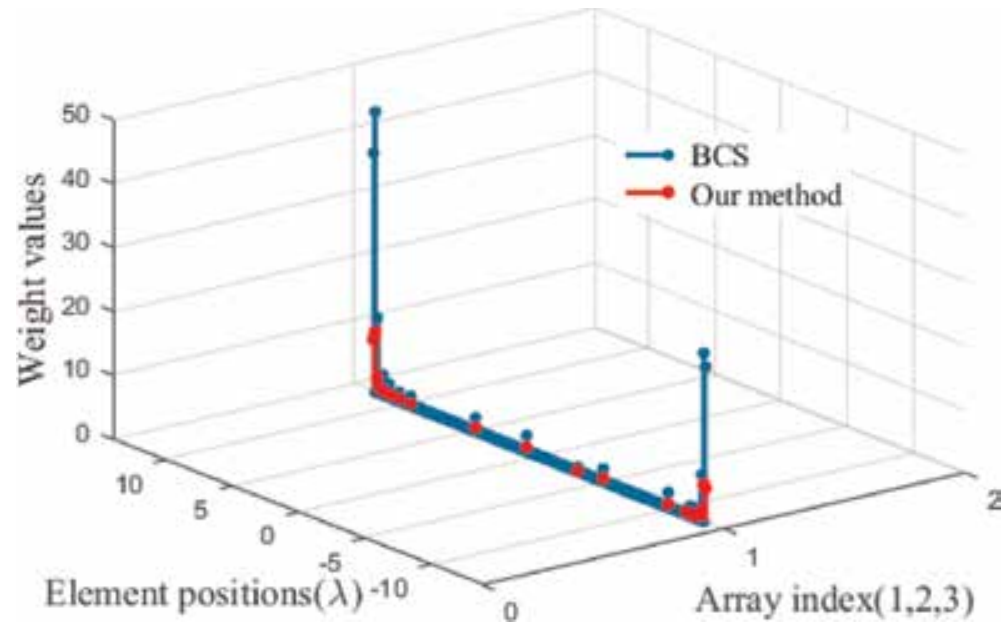

Figure 10.

Element positions and excitation amplitudes in an 18-element one-array orientation antenna.

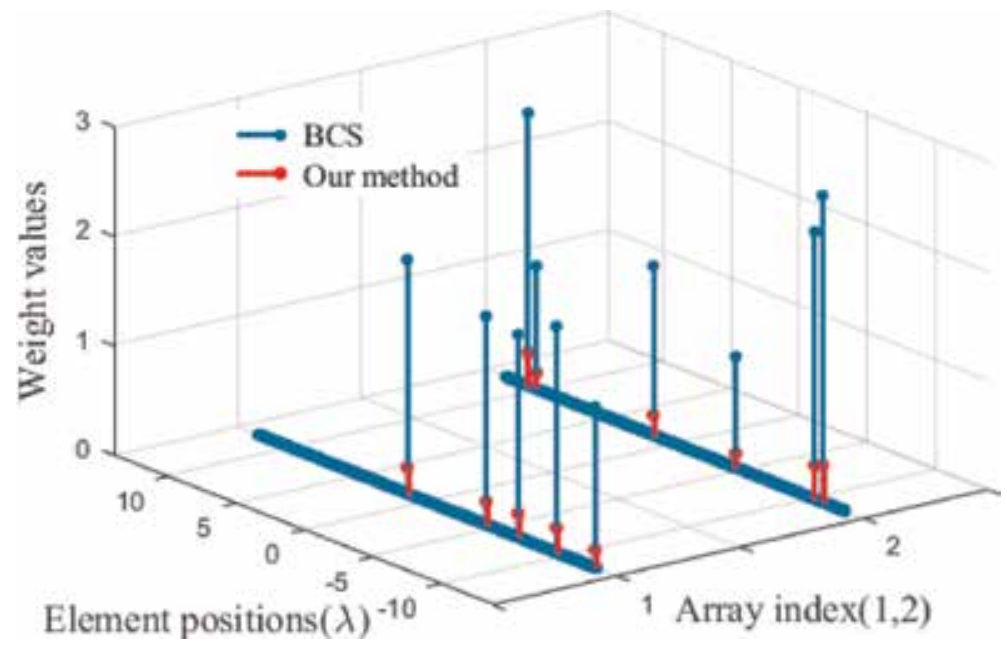

Figure 11.

Element positions and excitation amplitudes in an 11-element two-array orientation antenna. 


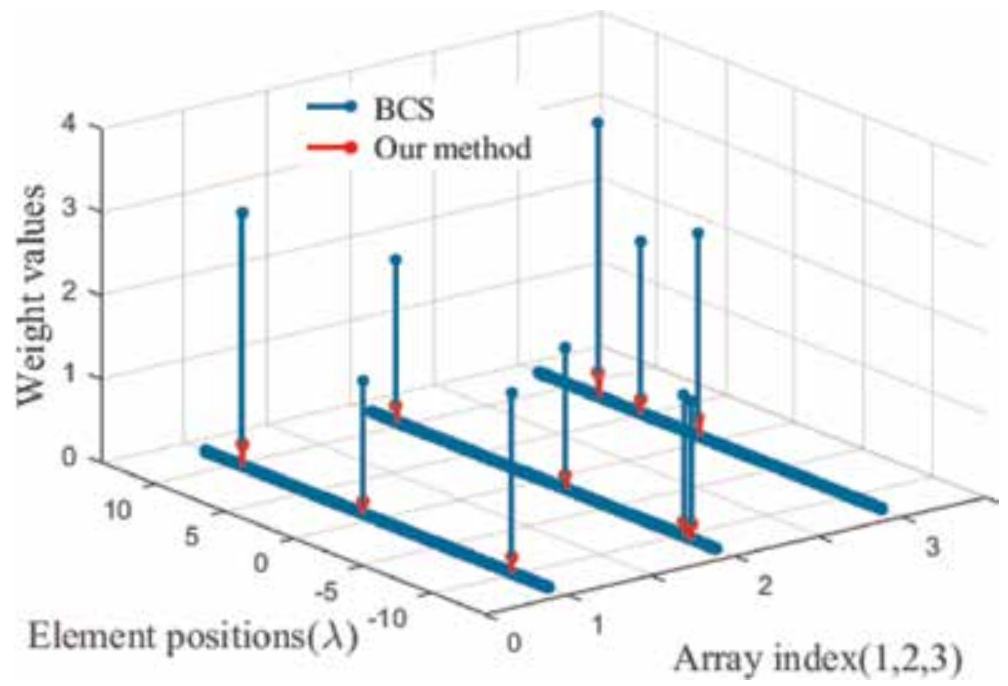

Figure 12.

Element positions and excitation amplitudes in a 9-element three-array orientation antenna.

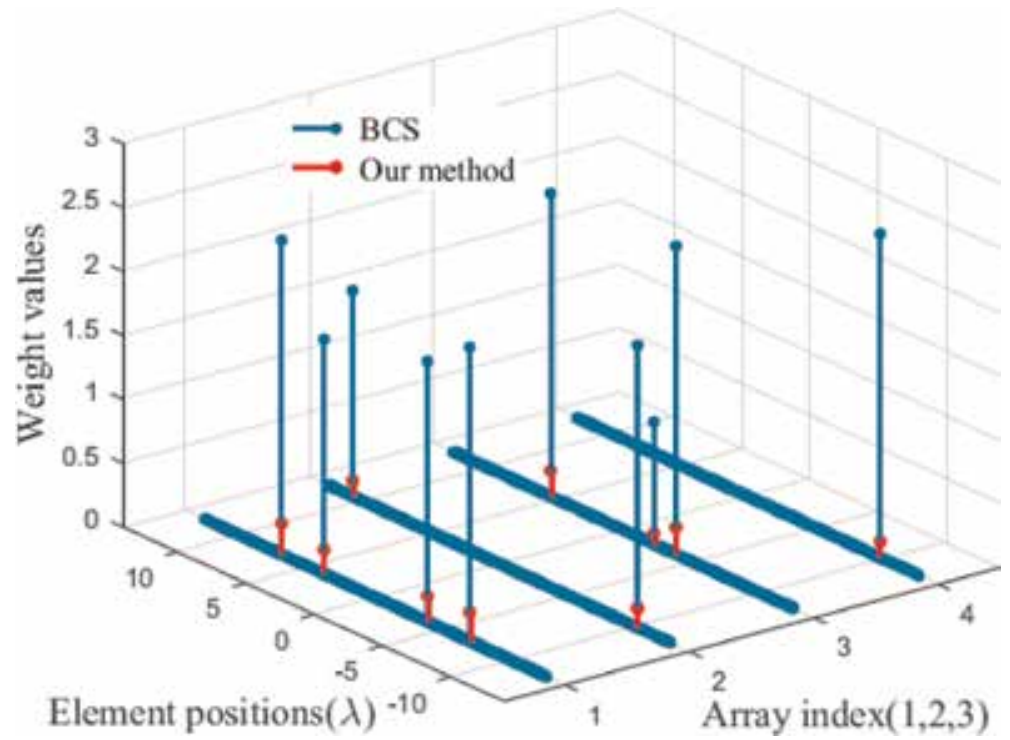

Figure 13.

Element positions and excitation amplitudes in a 10-element four-array orientation antenna.

The proposed APS algorithm based on reweighted $l_{1}$-norm minimization and array orientation diversity is demonstrated to be effective in reducing array elements, suppressing the sidelobe, and reducing the energy consumption to some extent.

\section{Conclusions}

This chapter focuses on the APS problem with sparse antenna array, which has practical applications, especially for massive antenna array. By using array 
orientation diversity and solving reweighted $l_{1}$-norm minimization convex optimization problem, the proposed APS algorithm shows the superiority in reducing array elements, suppressing the sidelobe, and reducing the energy consumption to some extent, and the robustness of the proposed design tool in real-life application will also be considered in our further work.

\section{Acknowledgements}

This work was supported in part by Sichuan Science and Technology Program (No. 18ZDYF2551) and in part by Fundamental Research Funds for the Central Universities (Program No. ZYGX2018J005).

\section{Author details}

Hui Chen* and Qun Wan

School of Information and Communication Engineering, University of Electronic Science and Technology of China, Chengdu, Sichuan, PR China

*Address all correspondence to: huichen0929@uestc.edu.cn

\section{IntechOpen}

(C) 2019 The Author(s). Licensee IntechOpen. This chapter is distributed under the terms of the Creative Commons Attribution License (http://creativecommons.org/licenses/ by/3.0), which permits unrestricted use, distribution, and reproduction in any medium, provided the original work is properly cited. (cc) BY 


\section{References}

[1] Dolph CL. A current distribution for broadside arrays which optimizes the relationship between beam width and side-lobe level. Proceedings of the IRE. 1946;34(6):335-348. DOI: 10.1109/ JRPROC.1946.225956

[2] Sarkar TK, Pereira O. Using the matrix pencil method to estimate the parameters of a sum of complex exponentials. IEEE Antennas and Propagation Magazine. 1995;37(1): 48-55. DOI: 10.1109/74.370583

[3] Jarske P, Saramaki T, Mitra SK, Neuvo Y. On properties and design of nonuniformly spaced linear arrays. IEEE Transactions on Acoustics, Speech, and Signal Processing. 1988;36(3):372-380. DOI: $10.1109 / 29.1534$

[4] Tseng C, Griffiths LJ. A simple algorithm to achieve desired patterns for arbitrary arrays. IEEE Transactions on Signal Processing. 1992;40:

2737-2746. DOI: 10.1109/78.165660

[5] Ng BP, Er MH, Kot C. A flexible array synthesis method using quadratic programming. IEEE Transactions on Antennas and Propagation. 1993;41: 1541-1550. DOI: $10.1109 / 8.267354$

[6] Haupt RL. Antenna Arrays: A Computational Approach. New Jersey: Wiley; 2010. DOI: 10.1002/ 9780470937464.ch5

[7] Zhou P, Ingram M. Pattern synthesis for arbitrary arrays using an adaptive array method. IEEE Transactions on Antennas and Propagation. 1999;47: 862-869. DOI: $10.1109 / 8.774142$

[8] Wang F, Yang R, Frank C. A new algorithm for antenna array pattern synthesis using recursive least square method. IEEE Transactions on Signal Processing Letter. 2003;10(8):235-238. DOI: $10.1109 /$ lsp.2003.814398
[9] Murino V, Trucco A, Regazzoni CS. Synthesis of unequally spaced arrays by simulated annealing. IEEE Transactions on Signal Processing. 1996;44(1):

119-123. DOI: $10.1109 / 78.482017$

[10] Khodier MM, Christodoulou CG. Linear array geometry synthesis with minimum side lobe level and null control using particle swarm optimization. IEEE Transactions on Antennas and Propagation. 2005;53(8): 2674-2679. DOI: 10.1109/

TAP.2005.851762

[11] Rattan M, Patterh MS, Sohi BS. Synthesis of aperiodic liner antenna arrays using genetic algorithm. In: Proceedings of the 2007 19th International Conference on Applied Electromagnetics and Communications; 24-26 September 2007; Dubrovnik, Croatia: IEEE; 2008. pp. 1-4

[12] Rahmat-Samii Y, Michielssen E. Electromagnetic Optimization by Genetic Algorithms. New Jersey: Wiley; 1999. DOI: 10.1364/IPR.1999.RTuE4

[13] Haupt RL, Werner DH. Genetic Algorithms in Electromagnetics. New Jersey: Wiley; 2007. DOI: 10.1109/ APS.1996.549878

[14] Lebret H, Boyd S. Antenna array pattern synthesis via convex optimization. IEEE Transactions on Signal Processing. 1997;45(3):526-532. DOI: $10.1109 / 78.558465$

[15] Wang F, Balakrishnan V, Zhou PY, Chen J, Yang R, Frank C. Optimal array pattern synthesis using semidefinite programming. IEEE Transactions on Signal Processing. 2003;51(5):1172-1183. DOI: 10.1109/TSP.2003.810308

[16] Liu Y, Nie Z, Liu Q. Reducing the number of elements in a linear antenna array by the matrix pencil method. IEEE 
Transactions on Antennas and

Propagation. 2008;56(9):2955-2962.

DOI: $10.1109 /$ tap.2008.928801

[17] Zhang WJ, Li L, Li F. Reducing the number of elements in linear and planar antenna arrays with sparseness constrained optimization. IEEE Transactions on Antennas and Propagation. 2011;59(8):

3106-3111. DOI: $10.1109 /$

TAP.2011.2158943

[18] Caratelli D, Vigano MC. A novel deterministic synthesis technique for constrained sparse array design problems. IEEE Transactions on Antennas and Propagation. 2011;59(11): 4085-4093. DOI: $10.1109 /$

TAP.2011.2164193

[19] Viani F, Oliveri G, Massa A. Compressive sensing pattern matching techniques for synthesizing planar sparse arrays. IEEE Transactions on Antennas and Propagation. 2013;61(9): 4577-4587. DOI: 10.1109/

TAP.2013.2267195

[20] Oliveri G, Bekele ET, Robol F, Massa A. Sparsening conformal arrays through a versatile BCS based method. IEEE Transactions on Antennas and Propagation. 2014;62(4):1681-1689. DOI: 10.1109/tap.2013.2287894

[21] Long-Jun LI, Wang BH, Xia CH. Synthesis of sparse conformal array antennas pattern. Acta Electronica Sinica. 2017;45(1):104-111. DOI: 10.3969/j.issn.0372-2112.2017.01.015

[22] Giorgio G, Luca T, Nicola A, Giacomo O, Paolo R. Sparse conformal array design for multiple patterns generation through Multi-Task Bayesian Compressive Sensing. In: Proceedings of 2017 IEEE International Symposium on Antennas and Propagation \& USNC/ URSI National Radio Science Meeting; 9-14 July 2017; San Diego, CA, USA: IEEE; 2017. pp. 429-430
[23] Bencivenni C, Ivashina MV, Maaskant R, Wettergren J. Synthesis of maximally sparse arrays using compressive sensing and full-wave analysis for global earth coverage applications. IEEE Transactions on Antennas and Propagation. 2016; 64(11):4872-4877. DOI: 10.1109/ TAP.2016.2594840

[24] D’Urso M, Prisco G, Tumolo RM. Maximally sparse, steerable, and non super directive array antennas via convex optimizations. IEEE Transactions on Antennas and Propagation. 2016;64(9):3840-3849. DOI: 10.1109/TAP.2016.2586490

[25] Tao H, Xiao-Pan S, Xue-Song L. Synthesis of sparse linear array for directional modulation via convex optimization. IEEE Transactions on Antennas and Propagation. 2018;66(8): 3959-3972. DOI: $10.1109 /$

TAP.2018.2835641

[26] Chen H, Shao H-Z, Wang W-Q. Joint sparsity-based range-angledependent beampattern synthesis for frequency diverse array. IEEE Access. 2017;5(99):15152-15161. DOI: 10.1109/ ACCESS.2017.2731973

[27] Chen H, Wan Q. Non-uniform array pattern synthesis using reweighted L1norm minimization method. AEUEInternational Journal of Electronics and Communications. 2013;67(9):795-798. DOI: 10.1016/j.aeue.2013.03.010

[28] Candès EJ, Wakin MB, Boyd SP. Enhancing sparsity by reweighted L1 minimization. Journal of Fourier Analysis and Applications. 2008;14(56):877-905. DOI: $10.1007 / \mathrm{s} 00041-008$ 9045-x

[29] Boyd S, Vandenberghe L, Faybusovich L. Convex optimization. IEEE Transactions on Automatic Control. 2006;51(11):1859-1859. DOI: 10.1109/TAC.2006.884922 
[30] Chen H, Wan Q. Rong fan:

Beampattern synthesis using reweighted L1-norm minimization and array orientation diversity. Radioengineering. 2013;22(2):602-609

[31] Grant M, Boyd S. CVX: Matlab software for disciplined convex programming, version 2.0 beta.

Available from: http://cvxr.com/cvx 


\title{
Reflectarray Pattern Optimization for Advanced Wireless Communications
}

\author{
Daniel Rodríguez Prado, Manuel Arrebola and \\ Marcos Rodríguez Pino
}

\begin{abstract}
A framework for the design and optimization of large dual-linear polarized, shaped-beam reflectarrays for advanced wireless communications is presented. The methodology is based on the generalized intersection approach (IA) algorithm for both phase-only synthesis (POS) and direct optimization of the reflectarray layout, as well as on the use of a method of moments in the spectral domain assuming local periodicity. A thorough description of the design and optimization procedures is provided. To demonstrate the capabilities of the proposed framework, two examples are considered. The first example is a shaped-beam reflectarray for future 5G base stations working in the millimeter waveband, radiating a sectored-beam pattern in azimuth and squared-cosecant pattern in elevation to provide constant power in the coverage area. The second example is a very large contoured-beam reflectarray for direct-to-home (DTH) broadcasting based on real mission requirements with Southern Asia coverage.
\end{abstract}

Keywords: array pattern synthesis, reflectarrays, optimization, wireless communications, 5G, base station, space communications, shaped-beam, contoured-beam, generalized intersection approach

\section{Introduction}

Wireless communication technologies have experienced a constant and rapid development over the past few decades. This has resulted in communication systems that need to fulfill increasing tighter requirements with the goal of improving their performance and quality. In particular, future developments and integration of 5G technologies for terrestrial and space communications [1] represent a great challenge. Specifically, the antenna is an important subsystem for wireless communications, since it is the device that converts the guided waves into propagating waves in free space and vice versa. Different parameters of the antenna may be optimized depending on the application, such as size, radiation pattern, matching, etc. In many cases, a shaped-beam pattern is necessary to adequately redirect power to the desired area. For instance, direct-to-home (DTH) applications need a contoured-beam footprint to match some specific geographic area on the surface of the Earth [2]. Also, an interesting feature for base stations for wireless communications is to provide constant power over a certain angular range. This may be achieved with a shaped-beam 
squared-cosecant pattern [3]. Traditionally, shaped parabolic reflectors or phased arrays have been employed for these applications [2, 3]. However, shaped parabolic reflectors are bulky and expensive to manufacture, while phased arrays require complex feeding networks which introduce high losses. Nonetheless, with the popularization of the microstrip technology, reflectarray antennas have become a potential substitute to parabolic reflector dishes and phased arrays.

The concept of reflectarray antenna was first introduced in 1963 [4] as a type of antenna that combines the simplicity of reflectors and the versatility of arrays, using waveguides as the reflecting element. This resulted in a bulky and expensive structure. However, reflectarrays were not widely studied until the development of low-profile printed antennas in the 1980s, when the printed planar reflectarray was developed [5]. It consists of an array of radiating elements that are spatially fed by a primary feed, which is usually a horn antenna. Its working principle is based on altering the properties of the electromagnetic field impinging from the feed. By adjusting the dimensions of the reflectarray elements, a phase shift is introduced in the impinging field [6], allowing to obtain the desired radiation pattern.

Although designing reflectarrays for high-gain pencil beam patterns at a certain direction may be achieved with analytical equations [5], the synthesis of noncanonical beams is a challenging task and requires the use of an optimization algorithm, especially in cases with tight requirements, such as space applications [2]. Since reflectarrays are usually comprised of hundreds or even thousands of elements, the employed algorithm must be computationally efficient. Until recently, the dominant approach was the phase-only synthesis (POS) [5], which employs a simplified analysis of the unit cell. This results in an extremely efficient synthesis [7-9] but has no control over the cross-polarization performance. The first approach to the crosspolar direct optimization of reflectarray antennas was presented in [10], using a method of moments based on local periodicity (MoM-LP) for the analysis of the unit cell. However, the algorithm was slow and only handled 1 polarization and small reflectarrays (225 elements). Other approaches for the minimization of the crosspolar component of the far field include a proper arrangement of the elements [11] and the minimization of the undesired tangential field adjusting the dimensions of the element [12] or through rotation [13]. These techniques are faster, but they work at the element level and thus provide suboptimal results.

In this chapter, we present a general framework for the efficient and accurate pattern optimization of reflectarray antennas for advanced wireless communications, including copolar and crosspolar specifications. It is based on the use of the generalized intersection approach (IA) algorithm [14] for the optimization and a MoM-LP [15] for the accurate characterization of the reflectarray unit cell. The design procedure is divided in several stages. First, a phase-only synthesis (POS) is carried out, to efficiently obtain the desired copolar pattern. Then, by using a zerofinding routine and the MoM-LP, the layout of the reflectarray is obtained adjusting the dimensions of each unit cell. Finally, an optional stage to improve the crosspolarization performance may be carried out. It employs the MoM-LP directly in the optimization loop to accurately characterize the crosspolar pattern. Both the POS and direct layout optimization are carried out with the generalized IA, demonstrating the versatility of the algorithm. Two relevant examples are provided to demonstrate the capabilities of the proposed framework. First, a shaped-beam reflectarray for future $5 \mathrm{G}$ base stations at millimeter waveband is proposed. It radiates a sectored-beam pattern in azimuth and a squared-cosecant pattern in elevation. The second example is a very large contoured-beam, spaceborne reflectarray for direct-to-home (DTH) broadcasting based on a real space mission.

The rest of the chapter is divided as follows. Section 2 introduces the optimization framework based on the generalized IA algorithm. Section 3 describes the 
design and optimization methodology using the generalized IA. Section 4 contains the results regarding the two reflectarray designs for advanced wireless communications. Finally, Section 5 contains the conclusions.

\section{Optimization framework for electrically large reflectarrays}

\subsection{Pattern requirements in the optimization procedure}

Before describing in detail the optimization algorithm, we will establish the different pattern requirements that can be imposed in the optimization procedure and how they are implemented in the generalized IA. For the case of radiation pattern optimization, the requirements may be imposed in the copolar and crosspolar components. When performing a POS, only copolar requirements are considered due to the simplifications in the analysis of the unit cell [9]. However, a direct optimization of the layout may consider both copolar and crosspolar requirements. In the generalized IA, the copolar requirements are given by means of two mask templates, which impose the minimum $\left(T_{\min }\right)$ and maximum $\left(T_{\max }\right)$ values that the far field must achieve. Thus, if $G_{\mathrm{cp}}$ is the copolar gain, it should fulfil

$$
T_{\min }(u, v) \leq G_{\mathrm{cp}}(u, v) \leq T_{\max }(u, v)
$$

where $u=\sin \theta \cos \varphi$ and $v=\sin \theta \sin \varphi$ are the angular coordinates where the far field is computed. Figure 1 shows an example of typical copolar requirement templates for a squared-cosecant pattern and a sectored-beam pattern in a plane, where $T_{\min }$ and $T_{\max }$ are the minimum and maximum specifications between which the copolar pattern must lie. Alternatively, these requirements can be provided in terms of minimum gain and maximum ripple.

On the other hand, there are several methodologies to implement crosspolar requirements. A typical approach is to minimize the crosspolar far field component by means of templates [16], similarly to the procedure followed with the copolar pattern. However, the crosspolar pattern does not need a lower bound in the optimization. Thus, only the maximum mask $T_{\max }^{\mathrm{xp}}$ is needed in this case, where the superscript indicates that the mask is applied to the crosspolar pattern, fulfilling

$$
G_{\mathrm{xp}}(u, v) \leq T_{\max }^{\mathrm{xp}}(u, v) .
$$

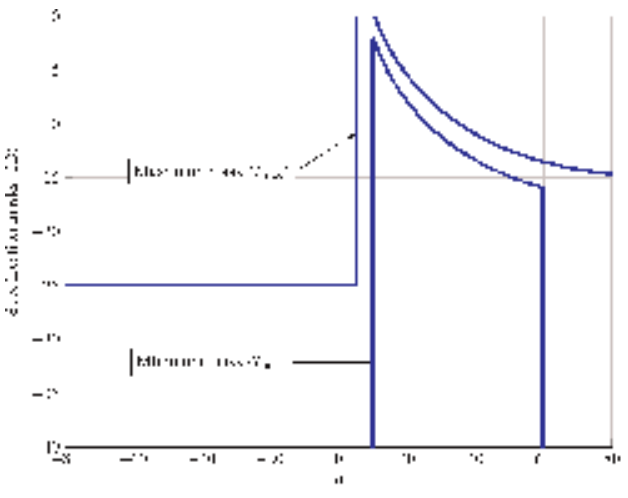

inil

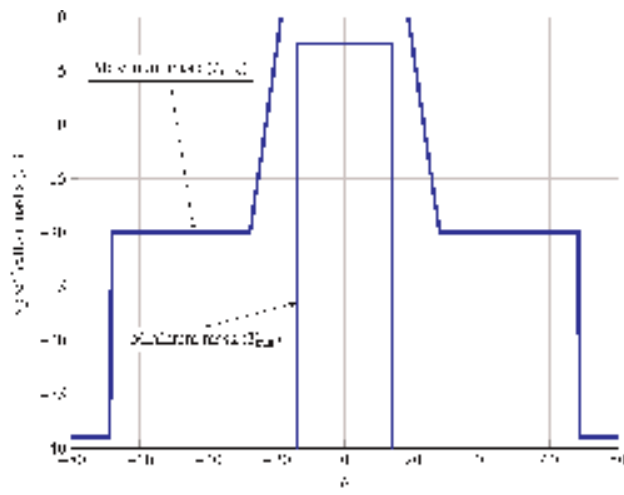

ith

Figure 1.

Typical requirement templates for (a) squared-cosecant pattern and (b) sectored-beam pattern. 
However, there are applications in which the figure of merit for crosspolarization performance is not the crosspolar pattern. In particular, some space missions [2] give the requirements for the crosspolar discrimination (XPD) and/or crosspolar isolation (XPI).

The XPD is defined for a certain coverage zone as the difference (in logarithmic scale) point by point of the copolar gain and the crosspolar gain. Mathematically it is expressed as

$$
\mathrm{XPD}(u, v)=G_{\mathrm{cp}}(u, v)-G_{\mathrm{xp}}(u, v), \quad(u, v) \in \Omega,
$$

where $\Omega$ is the coverage zone, XPD is in $\mathrm{dB}$ and $G_{\mathrm{cp}}$ and $G_{\mathrm{xp}}$ are in $\mathrm{dBi}$. Usually, the minimum XPD is considered, since it is the value limiting the XPD performance in the coverage zone:

$$
\mathrm{XPD}_{\min }=\min \{\mathrm{XPD}(u, v)\}, \quad(u, v) \in \Omega .
$$

Similarly, the XPI is defined for a certain coverage zone as the difference (in logarithmic scale) of the minimum copolar gain and the maximum crosspolar gain:

$$
\mathrm{XPI}=G_{\mathrm{cp}, \min }(u, v)-G_{\mathrm{xp}, \max }(u, v), \quad(u, v) \in \Omega,
$$

where XPI is in $\mathrm{dB}$ and $G_{\mathrm{cp}}$, min and $G_{\mathrm{xp}}$, max are in $\mathrm{dBi}$. Notice that, unlike the $\mathrm{XPD}$, the XPI is defined as a single value for a given coverage area. Also, the XPI is a stricter parameter than the XPD. Figure 2 shows graphically how the XPD and XPI are defined.

The optimization procedure should maximize the $\mathrm{XPD}_{\min }$ and/or XPI. Thus, if $T_{\mathrm{XPD}_{\min }}$ and $T_{\mathrm{XPI}}$ are the minimum requirement templates for $\mathrm{XPD}_{\min }$ and $\mathrm{XPI}$, respectively, they should fulfill the following condition:

$$
\begin{gathered}
T_{\mathrm{XPD}_{\min }} \leq \mathrm{XPD}_{\min }, \\
T_{\mathrm{XPI}} \leq \mathrm{XPI}
\end{gathered}
$$

\subsection{Generalized intersection approach}

The framework for the optimization of the radiation pattern of reflectarray antennas is based on the generalized intersection approach (IA) [4]. A flowchart of the algorithm is shown in Figure 3. It is an iterative algorithm which performs two operations at each iteration $i$ on the tangential field:

$$
\vec{E}_{\text {ref }, i+1}=\mathcal{B}\left[\mathscr{F}\left(\vec{E}_{\text {ref }, i}\right)\right],
$$

where $\vec{E}_{\text {ref }}$ is the tangential field on the reflectarray surface, calculated as

$$
\vec{E}_{\text {ref }}\left(x_{l}, y_{l}\right)=\mathbf{R}^{l} \vec{E}_{i n c}\left(x_{l}, y_{l}\right) \text {, }
$$

where $\left(x_{l}, y_{l}\right)$ are the coordinates of the centre of the reflectarray element $l, \vec{E}_{i n c}$ is the fixed incident field impinging from the feed and

$$
\mathbf{R}^{l}=\left(\begin{array}{cc}
\rho_{x x}^{l} & \rho_{x y}^{l} \\
\rho_{y x}^{l} & \rho_{y x}^{l}
\end{array}\right)
$$




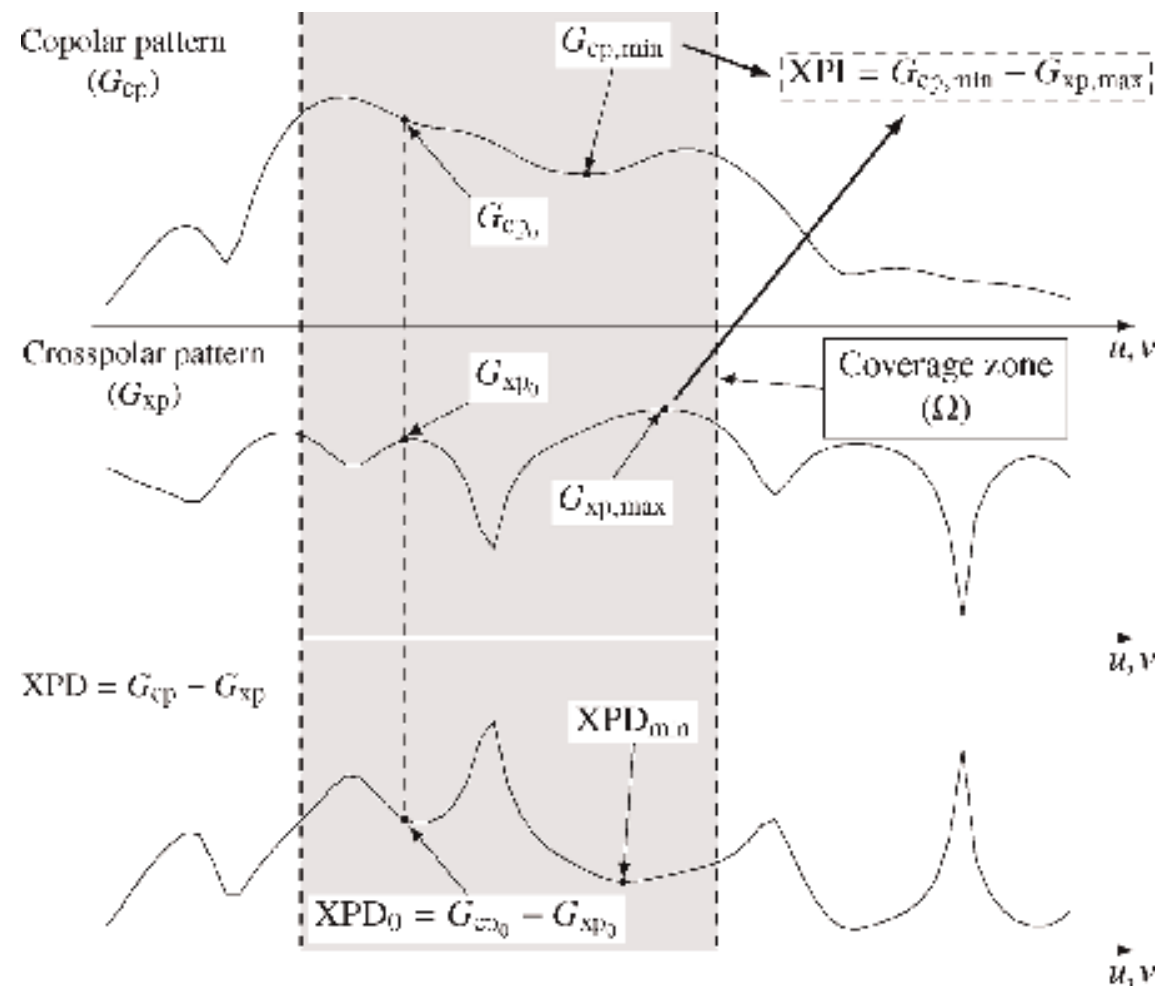

Figure 2.

Graphical definition of the parameters for co- and cross-polarization performance: The crosspolar discrimination (XPD), which is defined point by point as the difference between the copolar gain and the crosspolar gain, and the crosspolar isolation (XPI), which is defined for the coverage zone as the difference between the minimum copolar gain and the maximum crosspolar gain. The copolar and crosspolar patterns are in $d B i$, while the XPD and the XPI are in $d B$.

is the matrix of reflection coefficients which define the electromagnetic behaviour of the unit cell. These coefficients are complex numbers and are computed by a full-wave analysis tool assuming local periodicity [5]. $\rho_{x x}$ and $\rho_{y y}$ are known as the direct coefficients, while $\rho_{x y}$ and $\rho_{y x}$ are known as the cross-coefficients. In addition, the copolar pattern mainly depends on the direct coefficients phase, and the crosspolar pattern depends on all coefficients.

In (8), $\mathscr{F}$ is the forward projector. As shown in Figure 3, it is divided into two steps. First, starting from the tangential field, which depends on the optimizing variables, either the phases of the direct coefficients in a phase-only synthesis or the reflectarray element geometry in the case of a direct optimization, it computes the current far field radiated by the reflectarray. In its second step, it trims the far field according to the specification masks. For the power pattern synthesis, the specifications may be given in gain. Thus, if $G$ is the current gain of the reflectarray and $G^{\prime}$ the trimmed gain, then

$$
G^{\prime}(u, v)= \begin{cases}T_{\max }(u, v), & T_{\max }(u, v)<G(u, v) \\ T_{\min }(u, v), & G(u, v)<T_{\min }(u, v) \\ G(u, v), & \text { otherwise. }\end{cases}
$$

This operation is also applied to the crosspolar pattern when performing a direct optimization of the reflectarray layout. If the cross-polarization performance is 


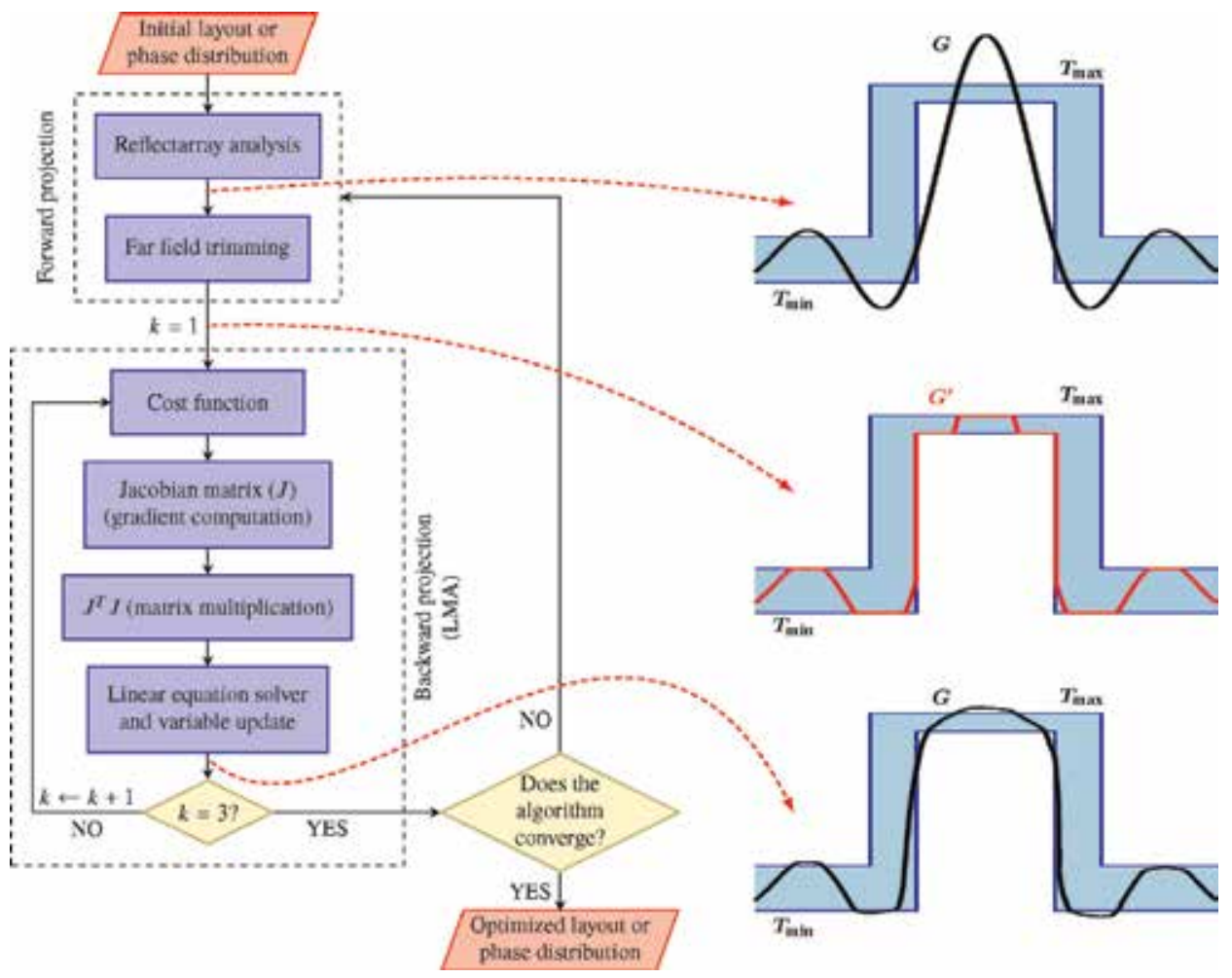

Figure 3.

Flowchart of the generalized intersection approach algorithm as applied to the far field in gain.

improved by means of the $\mathrm{XPD}_{\text {min }}$ or XPI optimization, a similar expression to Eq. (11) is used but only taking into account the minimum masks, as in Eqs. (6) and (7).

The second operation of the generalized IA, denoted by $\mathcal{B}$ in Eq. (8), is the backward projector. It minimizes the distance between the trimmed gain and the current gain radiated by the antenna (see Figure 3), obtaining a reflected tangential field that generates a radiation pattern that is closer to fulfil specifications:

$$
\vec{E}_{\text {ref }, i+1}=\mathcal{B}\left[\mathscr{F}\left(\vec{E}_{\text {ref }, i}\right)\right]=\min \operatorname{dist}\left[G_{i}, \mathscr{F}\left(\vec{E}_{\text {ref }, i}\right)\right] .
$$

The latter operation is performed by a general minimizing algorithm [14]. In addition, as a distance definition, we employ the Euclidean norm for squareintegrable functions [8], which is implemented by the weighted Euclidean metric:

$$
d_{i}=\operatorname{dist}^{2}\left(\mathscr{F}\left(\vec{E}_{\mathrm{ref}, i}\right), \quad G_{i}(u, v)\right)=\iint_{\Lambda} w(u, v)\left(G_{i}^{\prime}(u, v)-G_{i},(u v)\right)^{2} d u d v,
$$

where it was taken into account that the result of the forward projection is the trimmed gain in Eq. (11); $w(u, v)$ is a weighting function; and $\Lambda$ is a subset of the visible region $\left(u^{2}+v^{2} \leq 1\right)$ where the radiation pattern is optimized. The integral in Eq. (13) can be approximated by a sum for the points $(u, v)$ that belong to $\Lambda$ :

$$
d_{i}=\sum_{u, v \in \Lambda}\left[\sqrt{w(u, v) \Delta u \Delta v}\left(G_{i}^{\prime}(u, v)-G_{i},(u v)\right)\right]^{2} .
$$

This sum can be minimized by the Levenberg-Marquardt algorithm (LMA) [9]. 
Finally, the generalized IA can be applied to perform a phase-only synthesis (POS), where the optimizing variables are the phase shift introduced by each reflectarray element corresponding to the phases of the direct coefficients in Eq. (10), or a direct layout optimization, where the optimizing variables are the geometrical features of the unit cell.

\section{Design and optimization methodology}

This section briefly describes the design methodology employing the optimization framework presented in the previous section. It is applied to a reflectarray in single-offset configuration, as shown in Figure 4. The procedure is divided into three stages: first, a phase-only synthesis to obtain the desired radiation pattern; then, a design procedure to adjust the element dimensions yielding a reflectarray layout; and the last and optional stage is the optimization of the cross-polarization performance of the reflectarray antenna.

\subsection{Phase-only synthesis for the copolar pattern}

The first step in the design of a shaped-beam reflectarray antenna is a phaseonly synthesis (POS). The aim of the POS is to obtain a phase-shift distribution that generates the desired shaped radiation pattern, which in general cannot be obtained through analytical means since that approach presents some limitations [9]. Since we are interested in dual-linear polarized reflectarrays, two phase-shift distributions are necessary, one for each linear polarization. In addition, the generalized IA is a local search algorithm. Thus, a good starting point is of utmost importance.

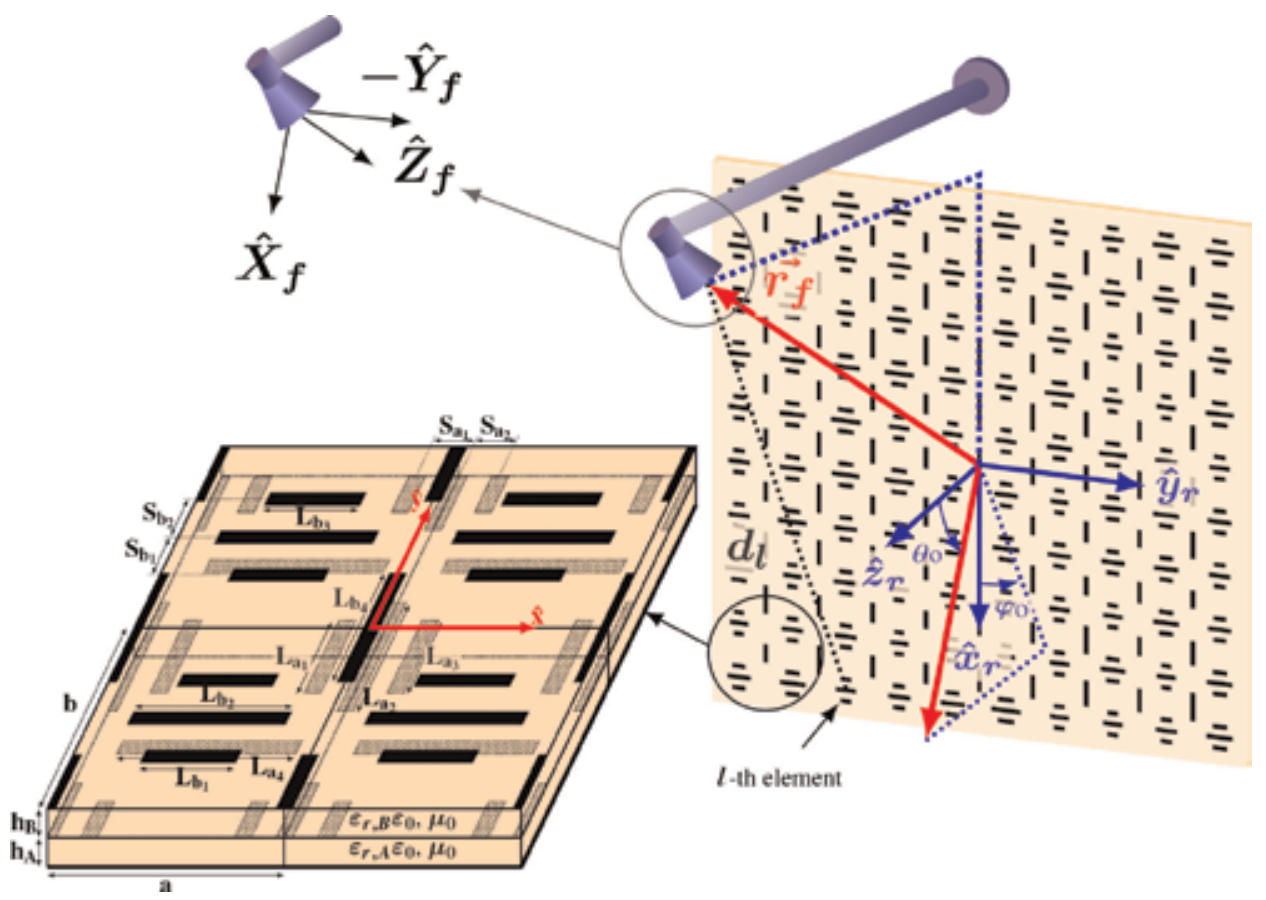

Figure 4.

Diagram of the planar reflectarray antenna optics and the considered unit cell based on two sets of parallel and coplanar dipoles. (C) 2018 IEEE. Reprinted, with permission, from [17]. 
It has been demonstrated that a properly focused pattern is sufficient for the POS [7]. In that case, the initial phase distribution for the POS may be obtained analytically [5]:

$$
\angle \rho\left(x_{l}, y_{l}\right)=k_{0}\left(d_{l}-\left(x_{l} \cos \varphi_{0}+y_{l} \sin \varphi_{0}\right) \sin \theta_{0}\right),
$$

where $\angle \rho\left(x_{l}, y_{l}\right)$ is the phase of a direct reflection coefficient ( $\rho_{x x}$ or $\rho_{y y}$, for linear polarizations $\mathrm{X}$ and $\mathrm{Y}$, respectively); $d_{l}$ is the distance from the feed to the $l$ th element (see Figure 4); and $\left(\theta_{0}, \varphi_{0}\right)$ is the pointing direction of the focused beam. The angle $\left(\theta_{0}, \varphi_{0}\right)$ is usually selected in a direction where the desired shaped beam has maximum gain.

Then, the generalized IA is employed to synthesize the desired pattern. For the POS, the elements are modelled as ideal phase shifters, in which there are no losses $\left(\left|\rho_{x x}\right|=\left|\rho_{y y}\right|=1\right)$ and no cross-polarization $\left(\rho_{x y}=\rho_{y x}=0\right)$. Thus, the matrix of reflection coefficients in (11) is simplified to

$$
\mathbf{R}^{l}=\left(\begin{array}{cc}
\exp \left(j \phi_{x x}^{l}\right) & 0 \\
0 & \exp \left(j \phi_{y y}^{l}\right)
\end{array}\right),
$$

where $\phi^{l}$ is the phase of the corresponding reflection coefficient. Thus, the optimizing variables are the phases of the direct coefficients. In addition, the POS is carried out in several steps, gradually increasing the number of optimizing variables as suggested in [14] to further improve the convergence of the algorithm. Once the desired phase-shift distributions are obtained, the following step is to obtain the reflectarray layout.

\subsection{Obtaining a reflectarray layout from a phase-shift distribution}

The procedure to obtain a reflectarray layout from the two phase-shift distributions obtained after the POS is summarized in the flowchart of Figure 5.



Figure 5.

Flowchart of the procedure to obtain a reflectarray layout from the synthesized phase-shift distribution for two linear polarizations. 
It requires the use of a full-wave technique based on local periodicity (FW-LP) to analyse the unit cell. Here, we employ the MoM-LP described in [18] to analyse the unit cell shown in Figure 4. In this step, a common procedure in the literature is to use a design curve obtained at normal incidence to seek the size of the reflectarray element that matches the required phase shift. However, it is recommended to consider the real angle of incidence to increase accuracy, especially for very large reflectarray antennas, since the phase shift varies with the angle of incidence [5].

This procedure is divided into three steps. Firstly, a phase-shift table is generated, increasing the size of the element (for instance, the patch size or dipole length) in little intervals. For the case at hand and using the unit cell based on two sets of parallel dipoles of Figure 4, two variables, $T_{x}$ and $T_{y}$, are defined that allow to control the phase shift for linear polarizations $\mathrm{X}$ and $\mathrm{Y}$, respectively. Thus, the phase-shift table is generated modifying at the same time $T_{x}$ and $T_{y}$. Then, we select two sizes of the element that provide a phase shift a little above and below the exact value. This is done independently for the two linear polarizations. Next, a linear equation is used to approximate the value of the element size that provides the required phase shift. Finally, by using a zero-finding routine (for instance, the Newton-Raphson method as indicated in Figure 5), the exact value for both polarizations is sought at the same time, taking into account the coupling between polarizations. This is done for every reflectarray element, obtaining a layout which generates the desired radiation pattern obtained in the POS of the first stage.

\subsection{Improvement of the cross-polarization performance through direct optimization}

The third and final stage is optional and consists in improving the crosspolarization performance of the synthesized reflectarray by directly optimizing its layout using a FW-LP tool. This is especially important for applications with tight cross-polarization requirements, such as space missions [2], since the layout obtained in the previous stage most likely will only comply with copolar specifications. As a starting point, the layout obtained in the previous stage is employed. Also, the copolar specification masks are maintained to keep the copolar pattern within specifications while the cross-polarization performance is improved.

There are a number of approaches that can be followed in this stage depending on the application. A common approach in the literature is to directly minimize the crosspolar component of the far field [19, 20]. This is done by applying Eq. (2) for the crosspolar pattern masks in the forward projector of the generalized IA. Another approach is to impose Eqs. (6) and (7) in order to maximize the $\mathrm{XPD}_{\text {min }}$ or the XPI. This is especially convenient for space applications in which cross-polarization requirements are specified by those parameters [17].

Nevertheless, this stage requires the use of a FW-LP tool to obtain the full matrix of reflection coefficients in Eq. (10) in order to correctly characterize the crosspolar radiation pattern. Thus, the improvement in cross-polarization performance will be slower than the POS in the first stage.

\section{Examples of application}

Here, we present two examples of application of the optimization framework presented in the previous sections. First, a medium-sized reflectarray is designed to work in a base station for future $5 \mathrm{G}$ application in the millimeter band at $28 \mathrm{GHz}$. The second example is a very large contoured-beam reflectarray for 
direct-to-home broadcasting in the $\mathrm{Ku}$-band at $12.5 \mathrm{GHz}$, based on a real mission with Southern Asia coverage.

\subsection{Reflectarray for $5 \mathrm{G}$ base station}

\subsubsection{Antenna specifications}

For the first example, the considered reflectarray is circular and comprised of 912 unit cells (34 elements in the main axes). The periodicity is $5.36 \mathrm{~mm}$ in both axes, which is half a wavelength at the working frequency, $28 \mathrm{GHz}$, in order to avoid grating lobes [5]. The feed is placed at $(-79.3,0.0,200.2) \mathrm{mm}$ with regard to the centre of the reflectarray (see Figure 4), and it is modelled as a $\cos ^{q} \theta$ function, with $q=20.6$, generating an illumination taper of $-14.6 \mathrm{~dB}$ at the reflectarray edges.

The unit cell shown in Figure 4 is used here. The separation between dipoles is set to $S_{a_{i}}=S_{b_{i}}=1 \mathrm{~mm}(i=1,2)$, while the width of all dipoles is set to $0.3 \mathrm{~mm}$. Variables $T_{x}$ and $T_{y}$ are defined as

$$
\begin{aligned}
& L_{a_{4}}=T_{x} ; \quad L_{b_{1}}=L_{b_{3}}=0.63 T_{x} ; \quad L_{b_{2}}=0.93 T_{x} \\
& L_{b_{4}}=0.95 T_{y} ; \quad L_{a_{1}}=L_{a_{3}}=0.58 T_{y} ; \quad L_{a_{2}}=T_{y} .
\end{aligned}
$$

The same substrate is used in both layers of the unit cell, with $\varepsilon_{r}=3.0$ and $\tan \delta=0.0010$, which corresponds to the commercially available Rogers R3003. In addition, the bottom layer has a height of $h_{A}=30 \mathrm{mil}=0.762 \mathrm{~mm}$, while the top layer has a height of $h_{B}=20 \mathrm{mil}=0.508 \mathrm{~mm}$. Figure 6 presents a unit cell study at central frequency, showing the phase shift produced by the reflectarray element as well as the losses. As it can be seen, the angular stability is good while having low losses better than $-0.3 \mathrm{~dB}$. Furthermore, the phase shift provided by the unit cell is more than $720^{\circ}$, which is more than enough for a reflectarray design and subsequent optimization.

Regarding the far field specifications, the chosen pattern for the 5G base station has a $30^{\circ}$ sectored beam in azimuth and a squared-cosecant beam in elevation to provide constant power flux in an elevation span of $50^{\circ}$.

\subsubsection{Results of the antenna design}

The starting point for the POS is a pencil beam pointing at $\left(\theta=10.4^{\circ}, \varphi=0\right)$. This direction corresponds to a region of the specification masks with high gain. To obtain this radiation pattern, the phase-shift distribution calculated with Eq. (15) is employed, and it is shown in Figure 7a for polarization $\mathrm{X}$, that is, for the direct
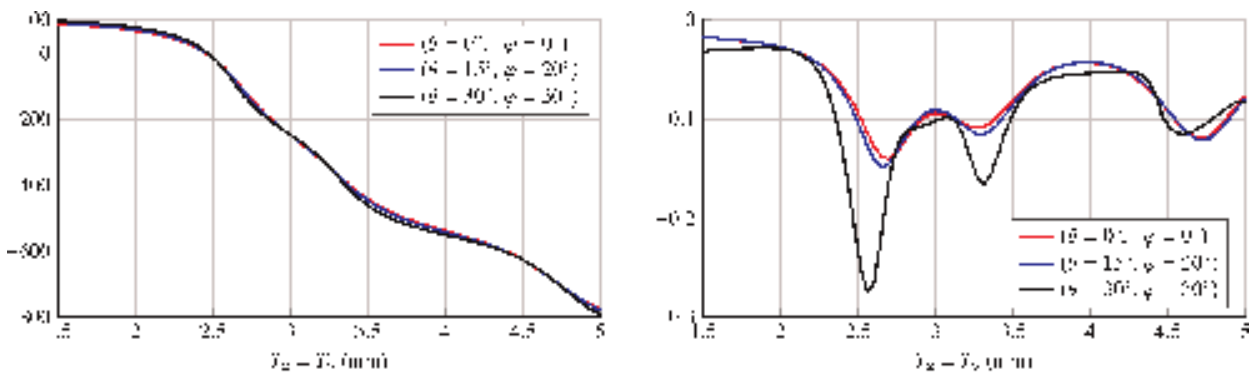

Figure 6.

Unit cell study for the reflectarray for $5 \mathrm{G}$ base station at $28 \mathrm{GHz}$ showing the phase shift (left) and the magnitude (right) for several angles of incidence. Unit cell presents a good angular stability with low losses. 




(a)

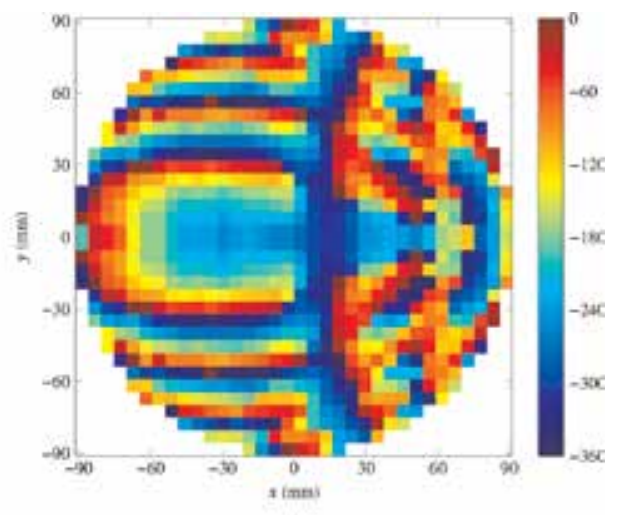

(b)

Figure 7.

For polarization X: (a) starting phase distribution (in degrees) obtained with Eq. (15) for the POS and (b) synthesized phase distribution (in degrees) after the POS with the generalized IA.

reflection coefficient $\rho_{x x}$ (the phase shift for polarization $\mathrm{Y}$ is the same). After the POS, the synthesized phase shift of Figure $7 \mathbf{b}$ is obtained, which generates the desired radiation pattern. Then, by using the procedure summarized in Figure 5, the reflectarray layout is found.

The obtained layout was simulated with a MoM-LP [18], and the resulting radiation pattern for polarization $\mathrm{X}$ is shown in Figure 8, where the copolar and crosspolar components of the far field are shown in the $u-v$ plane for the whole visible region. In this representation, it can be seen the sectored beam is along the $v$ axis for constant $u$, while along $u$ the squared-cosecant beam reduces the gain of the antenna from a maximum of $19.6 \mathrm{dBi}$ to roughly $5 \mathrm{~dB}$. This represents a dynamic range of almost $15 \mathrm{~dB}$ in which the shaped beam has to smoothly decrease over an angular span of $50^{\circ}$, making it challenging pattern to synthesize. In fact, it is very easy to obtain nulls in this region that penalize performance, even in simulations [21], and they have been avoided with success in the present example. Similar results were obtained for polarization Y.

On the other hand, Figure 9 represents the main cuts in elevation and azimuth for both linear polarizations along with the mask requirements. Here, it can be

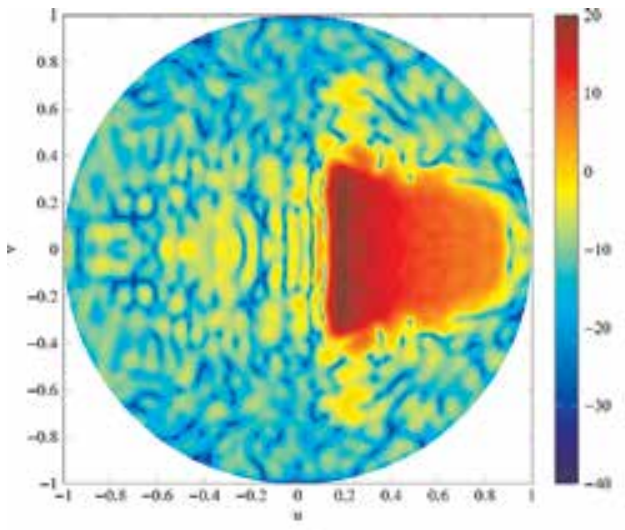

(a)

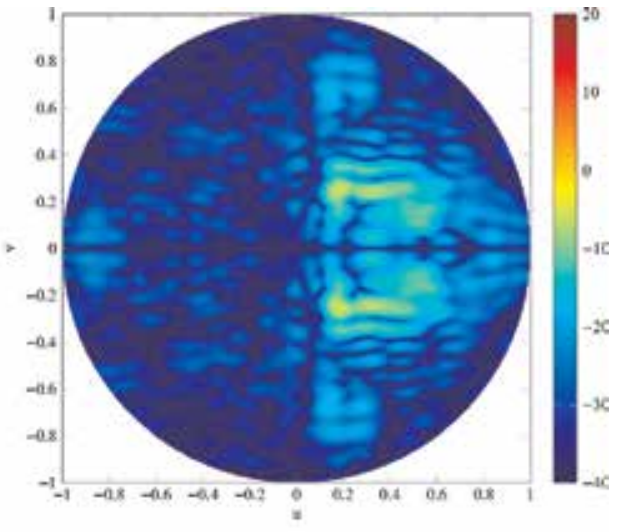

(b)

Figure 8.

Radiation pattern in the whole visible region radiated by the reflectarray designed for a $5 G$ base station for polarization X. (a) Copolar component of the far field. (b) Crosspolar component of the far field. 


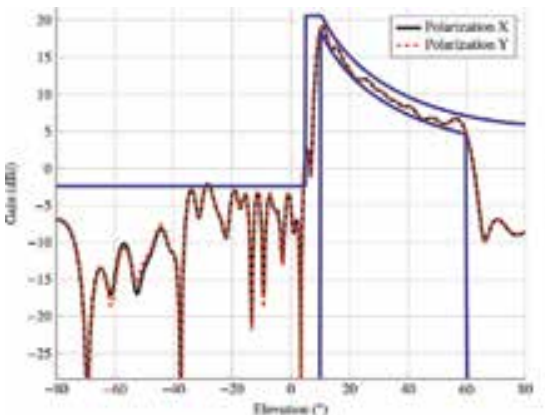

(a)

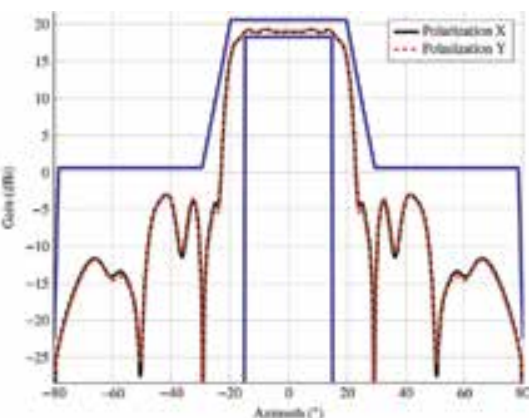

(b)

Figure 9.

Main cuts for both linear polarizations in (a) elevation and $(b)$ azimuth with the mask requirements for the reflectarray designed for a $5 G$ base station.

better appreciated how the specifications are met, with side lobes lower than $-2 \mathrm{~dB}$, which represent a SLL better than $20 \mathrm{~dB}$ for this shaped pattern. In addition, Figure 10 shows the radiation pattern in 3D perspective, along with a sketch of the reflectarray panel.

Regarding the cross-polarization performance, the initial design presents maximum crosspolar values of -6.1 and $-6.8 \mathrm{dBi}$ for polarizations $\mathrm{X}$ and $\mathrm{Y}$, respectively, while the maximum copolar gain is $19.6 \mathrm{dBi}$ for both polarizations. This gives a maximum copolar gain/maximum crosspolar gain ratio $\left(\mathrm{CP}_{\max } / \mathrm{XP}_{\max }\right.$ from here on) of 25.7 and $26.4 \mathrm{~dB}$ for polarizations $\mathrm{X}$ and $\mathrm{Y}$, respectively. The following step will be to improve the ratio $\mathrm{CP}_{\max } / \mathrm{XP}_{\max }$ by minimizing the crosspolar component of the far field while keeping the copolar pattern within specifications and maintaining the maximum copolar gain. To this end, a direct optimization layout will be performed using the generalized intersection approach. Now, the optimizing variables will be variables $T_{x}$ and $T_{y}$ as defined in Eq. (17), instead of the phases of the reflection coefficients. In addition, since the starting point already complies with the copolar requirements, all variables will be optimized at the same time.



Figure 10.

$3 D$ representation of the copolar component of the radiation pattern for a $5 G$ base station. 


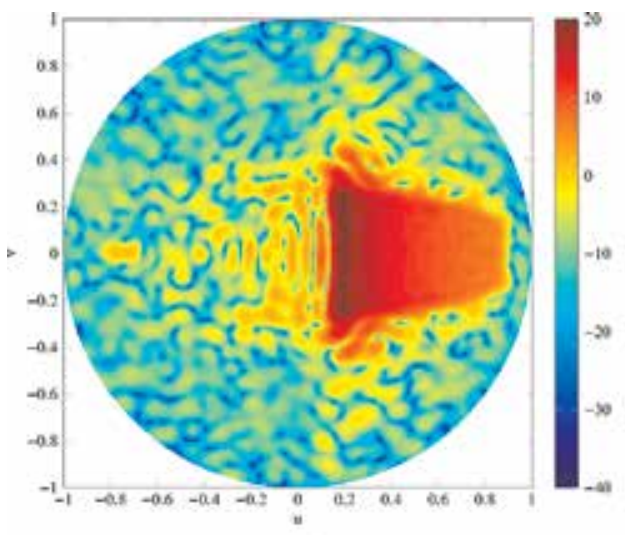

(a)

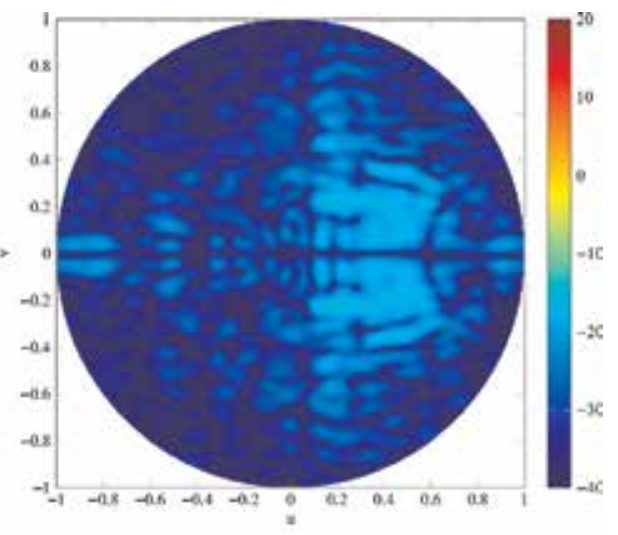

(b)

Figure 11.

Radiation pattern in the whole visible region radiated by the reflectarray designed for $5 G$ base station for polarization $X$ after the optimization to improve the cross-polarization performance: (a) copolar component of the far field and $(b)$ crosspolar component of the far field.

Thus, a total of 1824 variables will be considered. The copolar requirements are the same, while for the crosspolar pattern, a constant template $T_{\max }^{\mathrm{xp}} 40 \mathrm{~dB}$ below the maximum copolar gain is imposed for both linear polarizations. The goal is to minimize the crosspolar pattern as much as possible.

After the crosspolar optimization, the radiation pattern shown in Figure $\mathbf{1 1}$ was obtained for polarization X. When compared with the far field of Figure 8, it can be seen how the crosspolar pattern maximum value has been considerably reduced while keeping the copolar pattern within specifications. In fact, the maximum copolar gain is now $19.7 \mathrm{dBi}$ and $19.6 \mathrm{dBi}$ for polarizations $\mathrm{X}$ and $\mathrm{Y}$, respectively. At the same time, the maximum crosspolar values are -15.7 and $-15.4 \mathrm{dBi}$, with $\mathrm{CP}_{\text {max }}-\mathrm{XP}_{\text {max }}$ values of 35.4 and $35.0 \mathrm{~dB}$ for polarizations $\mathrm{X}$ and $\mathrm{Y}$, respectively. This represents an improvement of 9.7 and $8.6 \mathrm{~dB}$ for both linear polarizations. This information is summarized in Table 1. Finally, Figure 12 shows the layout of the optimized reflectarray for both layers.

\subsection{Reflectarray for direct-to-home satellite application}

\subsubsection{Antenna specifications}

For the second example, an elliptical reflectarray with axes $1128 \mathrm{~mm} \times 1080 \mathrm{~mm}$ and comprised of 6640 elements, is considered. The reflectarray cells are arranged in a rectangular grid of $94 \times 90$ elements for polarization $\mathrm{X}$ and $93 \times 89$ elements for polarization $\mathrm{Y}$, with a periodicity of $12 \mathrm{~mm}$ in both axes. The working frequency

\begin{tabular}{|c|c|c|c|c|c|c|}
\hline & \multicolumn{3}{|c|}{ Polarization X } & \multicolumn{3}{|c|}{ Polarization Y } \\
\hline & $\mathrm{CP}_{\max }$ & $\mathbf{X P}_{\max }$ & $\mathbf{C P}_{\max }-\mathbf{X P}_{\max }$ & $\mathrm{CP}_{\max }$ & $\mathbf{X P}_{\max }$ & $\mathrm{CP}_{\max -} \mathrm{XP}_{\max }$ \\
\hline Initial design & 19.6 & -6.12 & 25.7 & 19.6 & -6.84 & 26.4 \\
\hline Optimized design & 19.7 & -15.75 & 35.4 & 19.6 & -15.40 & 35.0 \\
\hline
\end{tabular}

Table 1.

For the reflectarray for $5 G$ base station, summary of the performance of the initial and optimized designs regarding the maximum copolar gain $\left(C P_{\max }\right)$, the maximum crosspolar gain $\left(X P_{\max }\right)$ and the difference between them $\left(C P_{\max }-X P_{\max }\right)$ for both linear polarizations. 


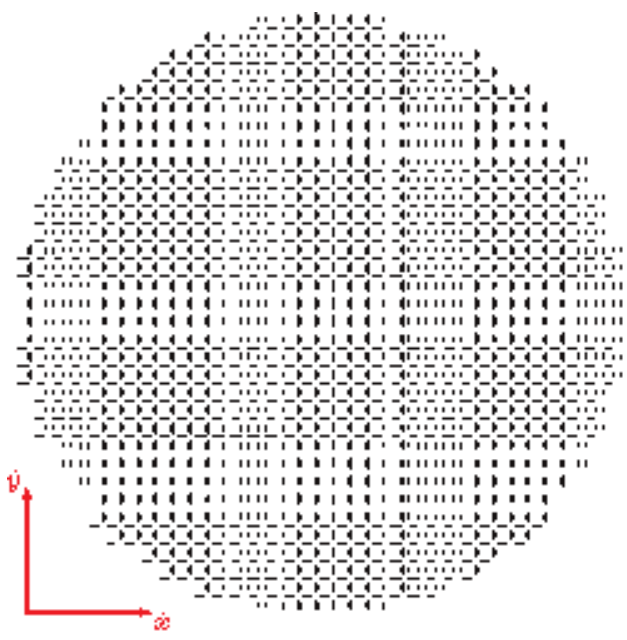

!ิ



!̣l?

Figure 12.

Layout of the reflectarray designed for $5 \mathrm{G}$ base station that generates a radiation pattern with a $30^{\circ}$ sectored beam in azimuth and a squared-cosecant beam in elevation: (a) bottom layer and (b) upper layer.

is $12.5 \mathrm{GHz}$. The feed is placed at $(-352.9,0.0,1061.7) \mathrm{mm}$ with regard to the reflectarray centre and is modeled as a $\cos ^{q} \theta$ with $q=18$, which generates an illumination taper of $-17.9 \mathrm{~dB}$.

A similar unit cell as in the previous example is used with different dimensions and materials. The separation between dipoles is now set to $S_{a_{i}}=S_{b_{i}}=2.5 \mathrm{~mm}$ $(i=1,2)$, while the width of all dipoles is set to $0.5 \mathrm{~mm}$ and $T_{x}$ and $T_{y}$ are defined in Eq. (17). Commercial substrates were chosen for both layers, the Arlon AD255C for the top layer, with $h_{A}=2.363 \mathrm{~mm}$ and $\varepsilon_{r}=2.17-j 0.0020$, and DiClad 880 for the bottom layer, with $h_{B}=1.524 \mathrm{~mm}$ and $\varepsilon_{r}=2.55-j 0.0036$. Figure 13 presents a unit cell study at central frequency, showing the phase shift produced by the reflectarray element as well as the losses. As it can be seen, the angular stability is good while having low losses better than $-0.3 \mathrm{~dB}$. The phase shift provided by the cell is slightly larger than $600^{\circ}$.

Figure 14 shows the contour requirements for the Southern Asia mission, similar to that provided by the SES-12 satellite. Zone 1 includes India, Nepal, Bhutan, Bangladesh and Sri Lanka, while zone 2 includes Pakistan and Afghanistan. According to the official specifications [22], the satellite provides an EIRP of 52
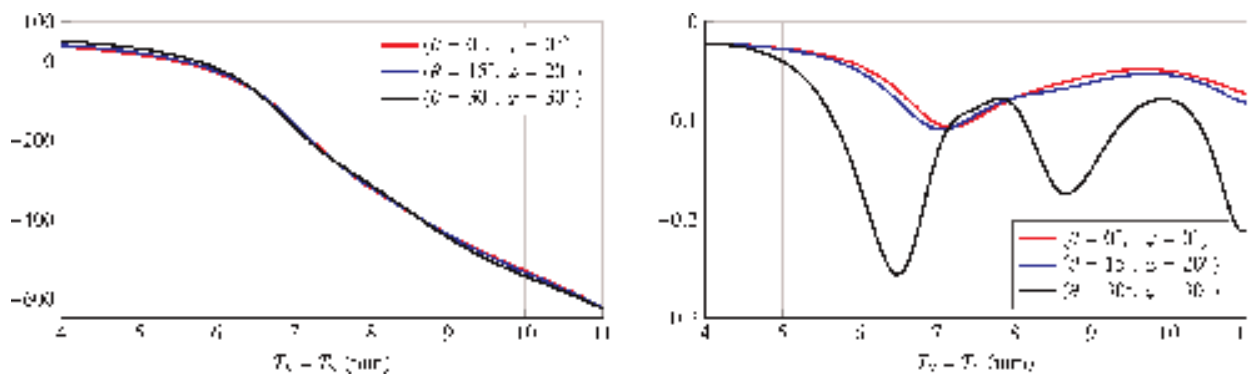

Figure 13.

Unit cell study for the reflectarray for DTH at $12.5 \mathrm{GHz}$ showing the phase shift (left) and the magnitude (right) for several angles of incidence. Unit cell presents a good angular stability with low losses while providing more than $600^{\circ}$ of linear phase shift. 




Figure 14 .

Footprint of the southern Asia coverage for direct-to-home broadcasting application. Zone 1 includes India, Nepal, Bhutan, Bangladesh and Sri Lanka, while zone 2 includes Pakistan and Afghanistan. This coverage mimics the one provided by the SES-12 satellite, placed in geostationary orbit at $95^{\circ} \mathrm{E} .(u, v)$ coordinates are in the satellite coordinate system.

$\mathrm{dBW}$ for zone 1 and $48 \mathrm{dBW}$ for zone 2 . The EIRP can be converted into gain using the following expression:

$$
G(\mathrm{dBi})=\operatorname{EIRP}(\mathrm{dBW})-P_{t}(\mathrm{dBW}),
$$

where $P_{t}$ is the power of the transponder. Assuming $P_{t}=150 \mathrm{~W}$, it gives a gain specification of $30 \mathrm{dBi}$ for zone 1 and $26 \mathrm{dBi}$ for zone 2 . In addition, the design process will take into account typical pointing errors $\left(0.1^{\circ}\right.$ in roll and pitch and $0.5^{\circ}$ in yaw). The design will be carried out in dual-linear polarization, imposing the same specifications in both polarizations.

\subsubsection{Results of the antenna design}

For the first step, a POS is carried out to obtain the desired copolar pattern in dual-linear polarization. Figure 15a shows the initial phase shift for the POS

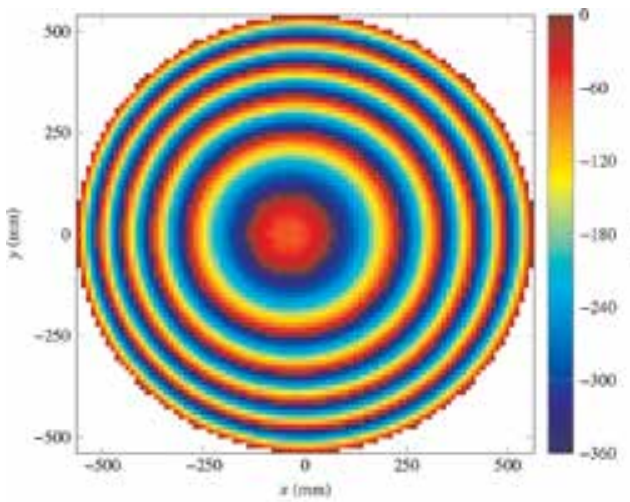

(a)

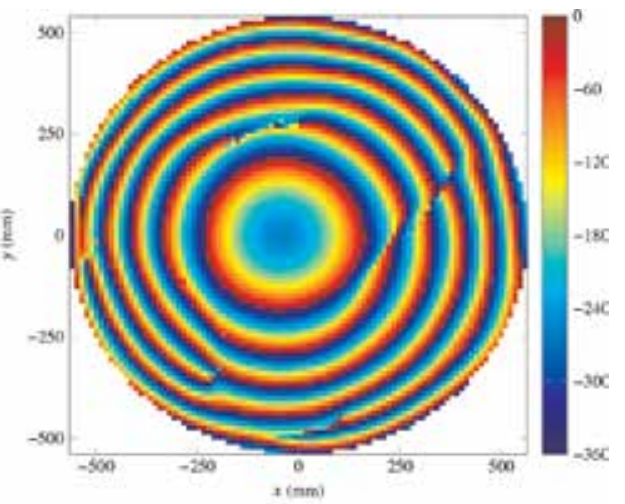

(b)

Figure 15.

For polarization X: (a) starting phase distribution (in degrees) obtained with Eq. (15) for the POS and (b) synthesized phase distribution (in degrees) after the POS with the generalized IA. 
obtained with Eq. (15). It generates a focused beam in the direction $\left(\theta=16.5^{\circ}\right.$, $\varphi=0.0^{\circ}$, which corresponds to a high-gain area in India. After the synthesis, the phases shown in Figure 15b were obtained for polarization X. The phases for polarization $\mathrm{Y}$ are similar. Once the layout has been obtained, the radiation patterns were computed using a MoM-LP tool. The copolar and crosspolar components for this initial design are shown in Figure 16 for polarization $\mathrm{X}$. In this case, the minimum copolar gain is 31.5 and $28.6 \mathrm{dBi}$ for zones 1 and 2, respectively. Similar results were obtained for polarization $Y$. Thus, the initial design complies with the requirements in both linear polarizations.

Space missions usually impose very stringent cross-polarization requirements in the form of crosspolar discrimination (XPD) and crosspolar isolation (XPI) for the transmit and receive bands, respectively. Notice that according to the definitions of minimum XPD in Eq. (4) and the XPI in Eq. (5), the XPI is a more stringent parameter than the XPD min. The first row of Table 2 shows the values of $\mathrm{XPD}_{\text {min }}$ and XPI for both coverage zones and polarizations. The initial design presents values of those parameters between 29.5 and $33.0 \mathrm{~dB}$. The goal is thus to improve the cross-polarization performance of this reflectarray by performing a direct optimization of the layout. As in the previous case, $T_{x}$ and $T_{y}$ are considered as optimization variables. Thus, a total of 13,097 variables will be considered. In addition, instead of minimizing the crosspolar pattern as in the previous example, now the $\mathrm{XPD}_{\text {min }}$ and XPI will be optimized as detailed in [17]. To that end, minimum masks of $37 \mathrm{~dB}$ are imposed for both parameters. The goal is to increase as much as possible the $\mathrm{XPD}_{\min }$ and XPI while keeping the minimum copolar gain for both coverage zones within specifications.

After the direct layout optimization, the cross-polarization performance of the reflectarray antenna significantly improved. The worst parameter is the XPI for zone 1 and polarization $\mathrm{X}$, which has a value of $37.5 \mathrm{~dB}$. It improved to $8 \mathrm{~dB}$ over the value for the initial design. The minimum improvement was $6 \mathrm{~dB}$ for the $\mathrm{XPI}$ for zone 1 and polarization $\mathrm{X}$ and $\mathrm{XPD}_{\min }$ for zone 2 and polarization $\mathrm{X}$. At the same time, the copolar minimum gain still complies with the specifications of $30 \mathrm{dBi}$ for zone 1 and $26 \mathrm{dBi}$ for zone 2 . A summary of the performance of the initial and optimized layout may be found in Table 2. In addition, Figure 17 shows the copolar and crosspolar pattern for polarization $\mathrm{X}$ of the optimized layout. Since the optimization has maximized the cross-polarization performance in the two coverage areas, the maximum crosspolar values are outside both of them.

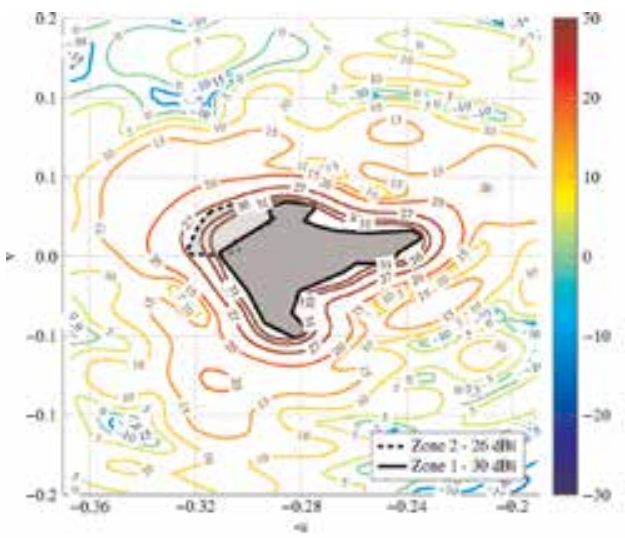

(a)



(b)

Figure 16.

Radiation pattern of the initial layout with southern Asia coverage for polarization X: (a) copolar pattern and (b) crosspolar pattern. 
Reflectarray Pattern Optimization for Advanced Wireless Communications DOI: http://dx.doi.org/10.5772/intechopen.88909

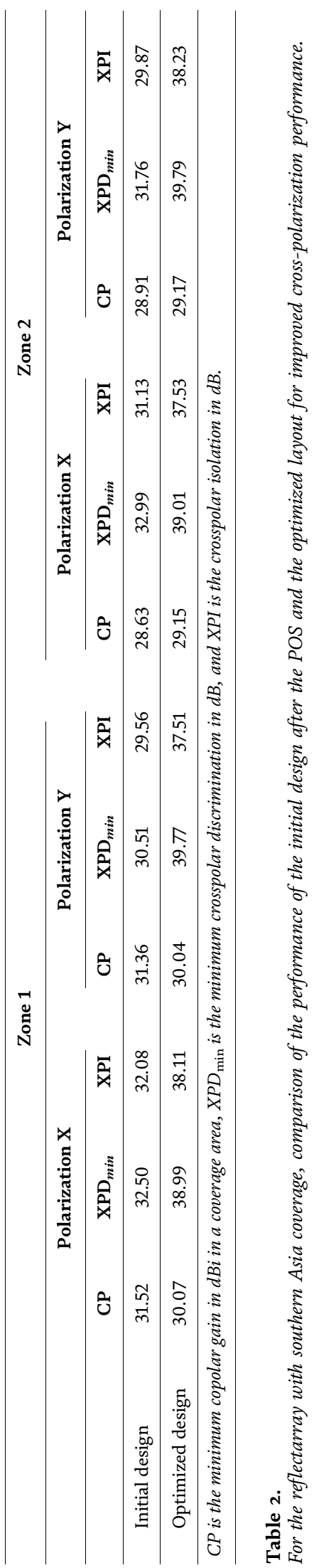




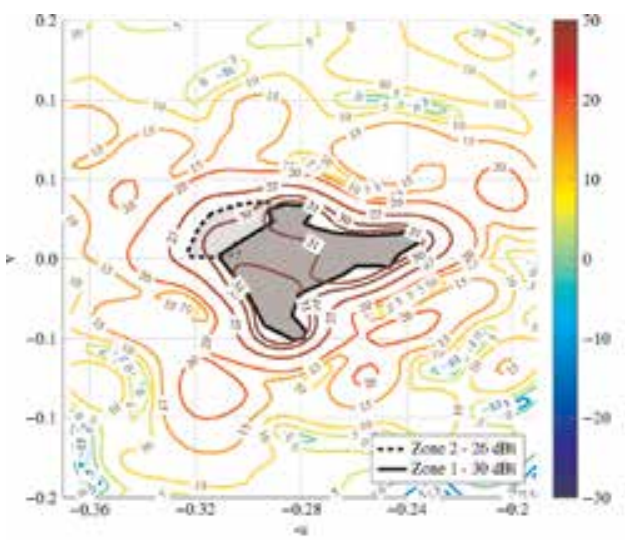

(a)

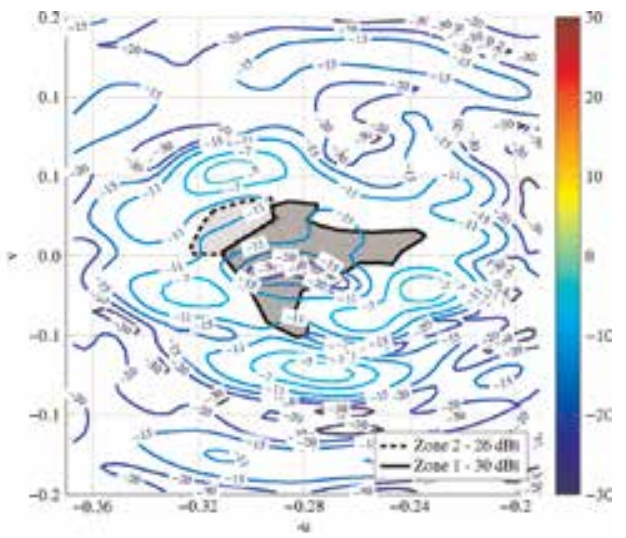

(b)

Figure 17.

Radiation pattern of the optimized layout with southern Asia coverage for polarization $X$ with improved cross-polarization performance: (a) copolar pattern and (b) crosspolar pattern.

Nevertheless, even its value has decreased, as it can be seen by comparing the crosspolar pattern of Figures 16 and 17.

\section{Conclusions}

A framework for the design and optimization of large dual-linear polarized reflectarray antennas has been presented. It is based on the generalized intersection approach (IA) algorithm, which is used for both a phase-only synthesis to obtain the initial design and a direct optimization of the reflectarray layout for a subsequent optimization of the cross-polarization performance. The IA employs the LevenbergMarquardt algorithm in the backward projector and a method of moments based on local periodicity (MoM-LP) to accurately characterize the electromagnetic response of the unit cell.

In order to demonstrate the capabilities of the proposed framework, two examples for advanced wireless communication applications are provided. For the two designs, a unit cell that consists in two sets of parallel dipoles is employed. Each set of dipoles controls the phase shift of a linear polarization. The first example is a shaped-beam reflectarray for future $5 \mathrm{G}$ base stations, radiating a sectored beam in azimuth and a squared-cosecant beam in elevation. A circular reflectarray with a diameter of $182 \mathrm{~mm}$ and comprised of 912 elements was proposed. The shapedbeam reflectarray achieves a maximum gain of $19.6 \mathrm{dBi}$ and a SLL better than $20 \mathrm{~dB}$. In addition, the gain smoothly decreases its value in elevation a total of $15 \mathrm{~dB}$ over a tilt of $50^{\circ}$. This feature makes the synthesis of a squared-cosecant pattern a challenging task. Finally, the maximum value of the crosspolar pattern was reduced more than $8.5 \mathrm{~dB}$ for both linear polarizations after a direct layout optimization using MoM-LP directly in the optimization loop, while maintaining the copolar pattern within requirements.

The second example consists of a 1.1-metre reflectarray for direct-to-home (DTH) broadcasting application. A Southern Asia coverage footprint that emulates the requirements of the SES-12 satellite has been selected. This coverage presents two zones with different gain requirements: one comprising India, Nepal, Bhutan and Bangladesh with a $30 \mathrm{dBi}$ of minimum copolar gain requirement and another for Pakistan and Afghanistan with a requirement of $26 \mathrm{dBi}$. After a phase-only 
synthesis, the layout of the reflectarray was obtained using a zero-finding routine and simulated with a MoM-LP tool. The minimum copolar gain achieved in both linear polarizations is better than $31 \mathrm{dBi}$ for zone 1 , while it is better than $28 \mathrm{dBi}$ for zone 2. Then, a direct optimization of the layout with MoM-LP was carried out to improve the cross-polarization performance. Both the minimum crosspolar discrimination and crosspolar isolation improved at least $6 \mathrm{~dB}$ for both zones and linear polarizations while keeping the minimum copolar gain within requirements.

The results shown here demonstrate the versatility of the proposed framework for the design and optimization of reflectarrays, as well as the feasibility of this type of antenna for advanced wireless communications.

\section{Acknowledgements}

This work was supported in part by the Ministerio de Ciencia, Innovación y Universidades under the project TEC2017-86619-R (ARTEINE); by the Ministerio de Economía, Industria y Competitividad under the project TEC2016-75103-C2-1-R (MYRADA); by the Gobierno del Principado de Asturias/FEDER under the project GRUPIN-IDI/2018/000191; by the Gobierno del Principado de Asturias through the Programa "Clarín" de Ayudas Postdoctorales/Marie Curie COFUND under the project ACA17-09; and by Ministerio de Educación, Cultura y Deporte/Programa de Movilidad "Salvador de Madariaga" (Ref. PRX18/00424).

\section{Thanks}

The authors would like to thank Dr. R. Florencio, Prof. R. R. Boix and Prof. J. A. Encinar for providing the MoM-LP software for the analysis of the reflectarray cell.

\section{Author details}

Daniel Rodríguez Prado ${ }^{1}$, Manuel Arrebola ${ }^{2 *}$ and Marcos Rodríguez Pino ${ }^{2}$

1 Institute of Sensors, Signals and Systems, School of Engineering and Physical Sciences, Heriot-Watt University, Edinburgh, UK

2 Department of Electrical Engineering, Group of Signal Theory and Communications, Universidad de Oviedo, Gijón, Spain

*Address all correspondence to: arrebola@uniovi.es

\section{IntechOpen}

(C) 2019 The Author(s). Licensee IntechOpen. This chapter is distributed under the terms of the Creative Commons Attribution License (http://creativecommons.org/licenses/ by/3.0), which permits unrestricted use, distribution, and reproduction in any medium, provided the original work is properly cited. (c) BY 


\section{References}

[1] Giambene G, Kota S, Pillai P. Satellite-5G integration: A network perspective. IEEE Network. 2018;32(5): 25-31

[2] Imbriale WA, Gao S, Boccia L, editors. Space Antenna Handbook. Hoboken, NJ, USA: John Wiley \& Sons; 2012

[3] Bergmann JR, Hasselmann FJV, Pereira LCP, Branco MGC. Reflector antenna configurations for radio base stations in cellular communications. In: IEEE-APS Conference on Antennas and Propagation for Wireless Communications. Waltham, Massachusetts, USA; 1998. pp. 61-64

[4] Berry DG, Malech RG, Kennedy WA. The reflectarray antenna. IEEE Transactions on Antennas and Propagation. 1963;11(6):645-651

[5] Huang J, Encinar JA. Reflectarray Antennas. Hoboken, NJ, USA: John Wiley \& Sons; 2008

[6] Pozar DM, Metzler TA. Analysis of a reflectarray antenna using microstrip patches of variable size. Electronics Letters. 1993;29(8):657-658

[7] Zornoza JA, Encinar JA. Efficient phase-only synthesis of contoured-beam patterns for very large reflectarrays. International Journal of RF and Microwave Computer-Aided Engineering. 2004;14(5):415-423

[8] Bucci OM, Franceschetti G, Mazzarella G, Panariello G. Intersection approach to array pattern synthesis. IEE Proceedings: Microwaves, Antennas and Propagation. 1990;137(6):349-357

[9] Prado DR, Álvarez J, Arrebola M, Pino MR, Ayestarán RG, Las-Heras F. Efficient, accurate and scalable reflectarray phase-only synthesis based on the Levenberg-Marquardt algorithm.
Applied Computational

Electromagnetics Society Journal. 2015; 30(12):1246-1255

[10] Bucci OM, Capozzoli A, D’Elia G, Musto S. A new approach to the power pattern synthesis of reflectarrays. In: Proc. URSI International Symposium on Electromagnetic Theory (EMTS'04). Italy: Pisa; 2004. pp. 1053-1055

[11] Hasani H, Kamyab M, Ali M. Low cross-polarization reflectarray antenna. IEEE Transactions on Antennas and Propagation. 2011;59(5):1752-1756

[12] Tienda C, Encinar JA, Arrebola M, Barba M, Carrasco E. Design manufacturing and test of a dualreflectarray antenna with improved bandwidth and reduced crosspolarization. IEEE Transactions on Antennas and Propagation. 2013;61(3): 1180-1190

[13] Florencio R, Encinar JA, Boix RR, Pérez-Palomino G, Toso G. Cross-polar reduction in reflectarray antennas by means of element rotation. In: 10th European Conference on Antennas and Propagation (EuCAP). Davos, Switzerland; 2016. pp. 1-5

[14] Bucci OM, D’Elia G, Mazzarella G, Panariello G. Antenna pattern synthesis: A new general approach. Proceedings of the IEEE. 1994;82(3):358-371

[15] Florencio R, Encinar JA, Boix RR, Losada V, Toso G. Reflectarray antennas for dual polarization and broadband telecom satellite applications. IEEE Transactions on Antennas and Propagation. 2015;63(4):1234-1246

[16] Bucci OM, D’Elia G, Romito G. Power synthesis of conformal arrays by a generalised projection method. IEE Proceedings-Microwaves, Antennas and Propagation. 1995;142(6):467-471 
[17] Prado DR, Arrebola M. Effective

XPD and XPI optimization in

reflectarrays for satellite missions. IEEE

Antennas and Wireless Propagation

Letters. 2018;17(10):1856-1860

[18] Florencio R, Boix RR, Encinar JA.

Enhanced MoM analysis of the

scattering by periodic strip gratings in

multilayered substrates. IEEE

Transactions on Antennas and

Propagation. 2013;61(10):5088-5099

[19] Zhou M, Sørensen SB, Kim OS, Jørgensen E, Meincke P, Breinbjerg O.

Direct optimization of printed reflectarrays for contoured beam satellite antenna applications. IEEE Transactions on Antennas and Propagation. 2013;61(4):1995-2004

[20] Prado DR, Arrebola M, Pino MR, Florencio R, Boix RR, Encinar JA, et al. Efficient crosspolar optimization of shaped-beam dual-polarized reflectarrays using full-wave analysis for the antenna element characterization. IEEE Transactions on Antennas and Propagation. 2017;65(2): 623-635

[21] Carrasco E, Arrebola M, Encinar JA, Barba M. Demonstration of a shaped beam reflectarray using aperturecoupled delay lines for LMDS central station antenna. IEEE Transactions on Antennas and Propagation. 2008; 56(10):3103-3111

[22] SES-12's mission. Available from: https://www.ses.com/our-coverage/ satellites/365 [Accessed: 28 August 2019] 



\title{
Antenna Pattern Multiplexing for Enhancing Path Diversity
}

\author{
Masato Saito
}

\begin{abstract}
In this chapter, we show the concept of antenna pattern multiplexing (APM), which enhances path diversity gain and antenna pattern diversity reception in multipath rich fading environment. We discuss the types of antennas that achieve the APM, i.e., generating time-varying antenna pattern and the benefits of reducing antenna size and hardware cost. When electronically steerable passive array radiator (ESPAR) antenna is used, the benefits can be maximised. A model of receiving process is proposed for analysing the ergodic capacity of multiple-input multipleoutput (MIMO) systems using APM. We derive a model of received signals to analyse the system performance. The received signal in matrix form includes an equivalent channel matrix, which is a product of antenna pattern matrix, the channel coefficient vector for each output. Numerical results in terms of ergodic capacity show the comparable performances of the proposed MIMO with APM to the conventional MIMO systems; in particular, the number of arrival paths and the number of antenna pattern are sufficiently large. Also the ergodic capacity can be equivalent to that of the conventional MIMO systems when the average SNR per antenna pattern is constant among the virtual antennas.
\end{abstract}

Keywords: antenna pattern multiplexing, path diversity, single-input multiple-output, multiple-input multiple-output, capacity, multipath fading

\section{Introduction}

Multiple-input multiple-output (MIMO) systems have attracted much attention as a means to improve the capacity of wireless communications by increasing the number of antennas. However, implementing multiple antennas can be a problem, particularly in mobile terminals due to their space limitation. In this study, we focus on array antennas at the receiver to enhance the capacity.

To resolve the problem, several methods have been proposed to achieve multiple separate received signal components by using a single radio frequency (RF) frontend with electronically steerable passive array radiator (ESPAR) antennas [1]. The modulated scattering array antenna was proposed for diversity and MIMO receivers [2-6]. The antenna consists of an antenna element for receiving signals and several modulated scattering elements (MSEs) like ESPAR antennas. The impedance of an MSE can be modulated or changed by with an applied sinusoidal voltage of frequency $f_{\mathrm{s}}$ to the variable reactance element connected to the MSEs. Then, the antenna patterns can vary also in a sinusoidal manner and can make the received signal frequency-shift by $\pm f_{\mathrm{s}}$. The frequency-shifted components can be used for 
diversity reception. The virtually rotating antenna was proposed also to diversify the received signal components in the frequency domain $[7,8]$. The principle of setting diversity branches in this domain is similar to that of the modulated scattering array antenna. In the rotating antenna, a combination of reactance values, which generates a desired antenna pattern, is applied sequentially to the multiple reactance elements of the antenna to rotationally change the directivity of the antenna. Also investigated was an ESPAR antenna based on the diversity receiver whose antenna patterns are time variable in a sinusoidal manner and are suitable for the MIMO-orthogonal frequency-division multiplexing (OFDM) receiver [9]. Other researches have been studied on diversity and MIMO receivers with ESPAR antennas having periodically variable antenna patterns by both theoretical and experimental investigations [10-14]. They also investigated the reactance time sequence, which generates sinusoidal antenna patterns with suppressed higherorder harmonics $[15,16]$.

In the studies shown above, it can be seen that, instead of using a fixed antenna pattern that may satisfy some criteria, they constantly changed the antenna pattern to generate multiple received signal components in the frequency domain. In this study, we propose a concept of antenna pattern multiplexing (APM) for setting multiple virtual antennas at the same location without additional physical antenna elements. Since the proposed APM also periodically varies antenna patterns to build multiple diversity branches or virtual antennas, it may be possible to consider the APM as a generalised method of the previously mentioned related studies. In APM, instead of sinusoidal waveforms or a sum of sinusoidal waveforms with different frequencies, we apply the sum of a set of orthogonal code sequences as the waveform to change antenna patterns. Therefore, the received signal can be separated into code domains to exploit path diversity instead of using only the narrow frequency domain, which is the case for the previous studies.

We introduce an antenna pattern matrix that consists of coefficients for each code sequence for each direction of received paths. With the matrix, we can derive the received signals of MIMO systems that use APM-based receivers in a form similar to the signals of the conventional MIMO systems. The ergodic capacity for the MIMO systems with APM technique is also derived ${ }^{1}$. Numerical results show that the capacity can be improved by increasing the number of arrival paths and the number of virtual antennas when the coefficients of APM are randomly distributed.

\section{Types of antennas to achieve APM}

Before mathematically analysing APM, we discuss the antennas that could realise the proposed APM concept. In APM, several antenna patterns, which are orthogonal to each other in time domain, should be multiplexed in similar manner to code-division multiplexing (CDM) or OFDM. To do so, it is essential that such antennas can generate time-varying antenna patterns. As such antennas, we consider array antennas or ESPAR antennas are good candidates because both antennas can change the antenna pattern from moment to moment.

We show a conceptual figure illustrating conventional array antenna, array antenna with APM, and ESPAR antenna with APM from left to right for comparative purposes in Figure 1. In the figure, we set the number of antenna elements at three as an example. Each antenna model consists of four parts: antenna elements,

\footnotetext{
${ }^{1}$ A part of the derivation is given in the our previous papers for limited cases of antenna pattern multiplexing $[11,17]$.
} 


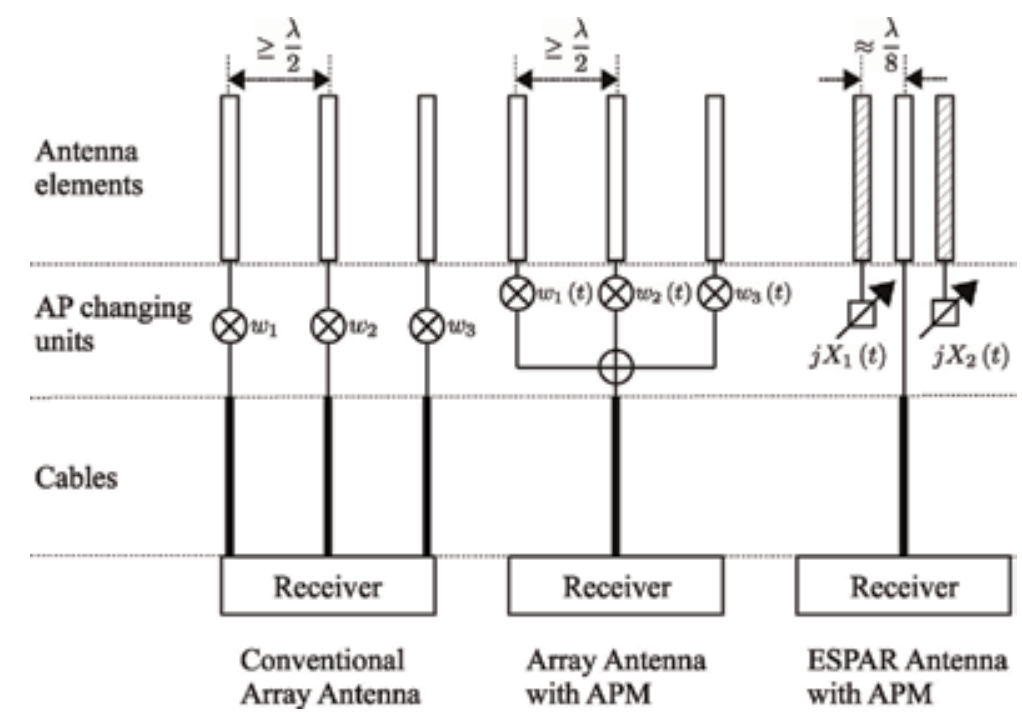

Figure 1.

The receiver models employing the conventional array antenna, array antenna with APM, and ESPAR antenna with APM of three antenna elements.

antenna pattern hanging units, cables between antenna elements and receivers, and receivers which receive signals through the cables.

In the part of antenna elements, the array antennas have three antenna elements connected to receivers, while ESPAR antenna has an element connected to the corresponding receiver and two parasitic elements which are connected to variable reactance components. In the figure, the shaded elements in ESPAR antenna show parasitic elements. In this part, the distance between neighbouring elements should be more than a half wavelength $\lambda / 2$ for array antennas to reduce the correlation between the received signals obtained by the elements. On the other hand, since ESPAR antennas form antenna patterns by exploiting mutual coupling between antenna elements, the neighbouring elements need to be sufficiently close to each other. A study on ESPAR antenna with six parasitic elements describes that $\lambda / 4$ is an appropriate distance [18]. In our previous work on two-element ESPAR antenna, appropriate distances between the elements are around $\lambda / 8$ [16]. Hence, ESPAR antennas can reduce the space required for antenna elements less than a half of the space of array antennas in the case of three antenna elements. The increase in the number of antenna elements provides more gains in terms of reducing antenna sizes for ESPAR antennas.

The antenna elements are connected to AP changing units, which are weight multiplication for array antennas and variable reactance elements (VREs) for ESPAR antennas. In the conventional array antenna, constant weights $w_{1}, w_{2}$, and $w_{3}$ are multiplied to form an antenna pattern based on a criteria such as maximising the signal-to-interference-plus-noise ratio (SINR) or minimising the interference. In array antenna with APM, the weights are functions of time $w_{1}(t), w_{2}(t)$, and $w_{3}(t)$, which form multiplexed AP and make the received signals travelling through antenna elements separable. Thus, we can add the signals and carry them to the receiver by a single cable. That is, the array antenna with APM can reduce the number of cables between antenna elements and the receiver and decrease their calibration cost. Since the parasitic elements do not connect to the receiver in ESPAR antenna, the cable cost can be also minimised. In ESPAR antenna, the antenna pattern or the directivity can be changed by the reactance values contributed by the parasitic elements. The reactance values of VREs can be changed by the 
voltage applying to the VREs. Thus, multiplexed antenna patterns can be generated by changing the reactance values $j X_{1}(t)$ and $j X_{2}(t)$ which are both time-varying functions. Since the relationship between reactance values and generated antenna patterns is nonlinear, even two reactance functions can make several or more than three multiplexed antenna patterns.

Note that, in this study, the objective of varying the weights of the antennas is not to control the antenna pattern or form a pattern that satisfies some criteria. We need to simply have the functionality of periodically time-varying antenna patterns.

As can be seen from the figure, by ESPAR antennas, we can reduce the size related to antenna elements and the number of cables. Hence, we have selected the ESPAR antennas as a good candidate for utilising APM [10-13, 15, 16]. However, one of the problems relevant to using ESPAR antennas is in its difficulty of designing antenna patterns and time-varying voltage waveform applying to VREs. The difficulty comes from the nonlinear processes of the conversions from voltage to reactance and from reactance to antenna pattern and their time-varying properties. Therefore, to find the optimal set of voltage waveform applying to VREs is an open problem.

\section{Modelling of antenna pattern multiplexing}

In this section, we build a model of the receiver with APM and mathematically derive the received signals in MIMO applications.

\subsection{Signals to change antenna pattern}

As we mentioned in the previous section, the antenna patterns can be changed by applying periodically time variable voltages to the VREs connected to parasitic antenna elements. Since the applied voltages are periodic function of time, we assume that the appeared antenna patterns are also periodic functions of time.

We define a periodic function of time, $a_{m}(t)$, whose period is $T_{\mathrm{s}}$. The function is assumed to be the weight for $m$-th antenna element for array antenna implementation and the reactance values of $m$-th VREs for ESPAR antenna implementation (see Figure 1). The function for a duration of the period $0 \leq t<T_{\mathrm{s}}$ is given by

$$
a_{m}(t)=\sum_{k=1}^{N_{\mathrm{a}}} b_{m, k} \cdot f_{k}(t),
$$

where $f_{k}(t)$ is the $k$-th function of a set of $N_{\mathrm{a}}$ orthonormal functions and $b_{m, k}$ is a complex-valued coefficient of $f_{k}(t)$ for $m$-th element. Since the functions $f_{k}(t)$ are orthogonal to each other, they have the following property:

$$
\frac{1}{T_{\mathrm{s}}} \int_{0}^{T_{\mathrm{s}}} f_{k}(t) \cdot f_{l}^{*}(t) \mathrm{d} t= \begin{cases}1 & (k=l) \\ 0 & (k \neq l)\end{cases}
$$

where $f_{l}^{*}(t)$ is the complex conjugate of $f_{l}(t)$. From the orthogonality shown in Eq. (2), we can derive another property for $k=l$ and assume a property for $k \neq l$ as

$$
f_{k}(t) \cdot f_{l}^{*}(t)=\left\{\begin{array}{ll}
1 & (k=l) \\
\exp \{j \Theta(t)\} & (k \neq l)
\end{array},\right.
$$


where $\Theta(t)$ is a uniform random process in the interval $[0,2 \pi)$. The conventional APM methods use the DC and sinusoids of one or several frequencies as $f_{k}(t)$ in Eq. (1). In comparison, in this study, we consider the function $f_{k}(t)$ to be a signal that is spread by using the spreading code sequence used in direct-sequence spread spectrum (DSSS) systems or code-division multiple access (CDMA) systems. Thus, we assume the function $f_{k}(t)$ can be expressed as

$$
f_{k}(t)=\sum_{l=1}^{N_{\mathrm{c}}} c_{l k} \cdot g\left(t-(l-1) T_{\mathrm{c}}\right),
$$

where $c_{l k}$ is the $l$-th chip of the waveform; $f_{k}(t)$ is assumed to have a complex value with a constant amplitude, $\left|c_{l k}\right|=1 / \sqrt{N_{c}} ; N_{c}$ is the number of chips in a period $T_{s}$; and $g(t)$ is the pulse waveform of a chip. In this paper, we assume that $g(t)$ is a rectangular pulse with duration $T_{\mathrm{c}}$ for simplicity. That is, $g(t)$ is shown as follows:

$$
g(t)=\left\{\begin{array}{lc}
1 & \left(0 \leq t<T_{\mathrm{c}}\right) \\
0 & \text { otherwise }
\end{array}\right.
$$

The product of two functions in Eq. (3) for $0 \leq t<T_{\mathrm{s}}$ can be rewritten as follows:

$$
\begin{aligned}
f_{k}(t) \cdot f_{l}^{*}(t) & =\sum_{m=1}^{N_{\mathrm{c}}} c_{m k} \cdot c_{m l}^{*} \cdot g\left(t-(m-1) T_{\mathrm{c}}\right) \cdot g^{*}\left(t-(m-1) T_{\mathrm{c}}\right) \\
& = \begin{cases}1 & (k=l) \\
\sum_{m=1}^{N_{\mathrm{c}}} c_{m k} \cdot c_{m l}^{*} \cdot g\left(t-(m-1) T_{\mathrm{c}}\right) & (k \neq l)\end{cases}
\end{aligned}
$$

As we can see from Eq. (7), the product can be shown by the product of only chips consisting of $f_{k}(t)$ and $f_{l}(t)$. Then, we consider a discrete time expression of $f_{k}(t)$ by introducing vector $\boldsymbol{c}_{k}$ whose components are the chips of $f_{k}(t)$. The vector can be given as

$$
\boldsymbol{c}_{k}=\left(\begin{array}{llll}
c_{1 k} & c_{2 k} & \cdots & c_{N_{\mathrm{c}} k}
\end{array}\right)^{\mathrm{T}},
$$

where $^{\mathrm{T}}$ is a transpose operator. Then, we obtain a code matrix, $C$, by aligning the vectors as follows:

$$
\begin{aligned}
& C=\left(\begin{array}{llll}
c_{1} & c_{2} & \cdots & c_{N_{\mathrm{a}}}
\end{array}\right) \\
& =\left(\begin{array}{cccc}
c_{11} & c_{12} & \cdots & c_{1 N_{\mathrm{a}}} \\
c_{21} & c_{22} & \cdots & c_{2 N_{\mathrm{a}}} \\
\vdots & \vdots & \ddots & \vdots \\
c_{N_{\mathrm{c}} 1} & c_{N_{\mathrm{c}} 2} & \cdots & c_{N_{\mathrm{c}} N_{\mathrm{a}}}
\end{array}\right)
\end{aligned}
$$

Since the orthogonality between two functions shown in Eq. (2) is satisfied, the following property of $C$ can be derived:

$$
C^{\mathrm{H}} C=I_{N_{\mathrm{a}}}
$$

where ${ }^{\mathrm{H}}$ is an Hermitian transpose operator and $I_{N_{\mathrm{a}}}$ is the identity matrix of size $N_{\mathrm{a}} \times N_{\mathrm{a}}$. 
The waveform $a_{m}(t)$ of Eq. (1) can be shown in a discrete time expression in matrix form as $C \boldsymbol{b}$ by setting a vector, $\boldsymbol{b}=\left(\begin{array}{lll}b_{0} & b_{1} \cdots b_{N_{\mathrm{a}}}\end{array}\right)^{\mathrm{T}}$.

\subsection{Received signals at receiver with APM}

In the proposed APM, we apply signal $a_{m}(t)$ given in Eq. (1) to the antenna pattern changing units. Here, we assume that the mapping from the signals $a_{m}(t)$ to the antenna patterns is a linear map ${ }^{2}$. In other words, the generated antenna patterns can be shown in a linear combination of $f_{l}(t)$ for $l=1, \ldots, N_{\mathrm{a}}$.

Then, we consider the antenna pattern for a given direction. Suppose that a ball surrounds the entire receive antenna. On the ball, the $p$-th received signal path sent by $l$-th transmit antenna arrives at point $\left(\phi_{l p}, \theta_{l p}\right)$, where $\phi_{l p}$ is an azimuth and $\theta_{l p}$ is an elevation from the origin of the ball, respectively. We assume that a periodically time-varying far-field antenna pattern, $d_{l p}\left(\phi_{l p}, \theta_{l p}, t\right)$, which the arrival path experiences, in an equivalent baseband expression can be given as

$$
d_{l p}\left(\phi_{l p}, \theta_{l p}, t\right)=\sum_{k=1}^{N_{\mathrm{a}}} d_{k l p}\left(\phi_{l p}, \theta_{l p}\right) \cdot b_{k} \cdot f_{k}(t),
$$

where $d_{k l p}\left(\phi_{l p}, \theta_{l p}\right)$ is the complex-valued coefficient of $f_{k}(t)$ for the direction of arrival path, which could be determined by the direction of the received signal, the structure of the antenna, and the waveforms applied to the antenna. Since the direction can change for each received signal, we assume $d_{k l p}\left(\phi_{l p}, \theta_{l p}\right)$ is a random variable, whose amplitude and phase follow a distribution that can be determined by the structure of the antenna and the waveforms applied to the antenna. In discrete time matrix form, Eq. (12) can be shown as $C B \boldsymbol{d}_{l p}$, where

$$
B=\operatorname{diag}(\boldsymbol{b})
$$

and $\operatorname{diag}(\boldsymbol{b})$ is a diagonal matrix whose diagonal components are given by $\boldsymbol{b}$ and $\boldsymbol{d}_{l p}=\left(d_{0 l p}\left(\phi_{l p}, \theta_{l p}\right) d_{1 l_{p}}\left(\phi_{l p}, \theta_{l p}\right) \ldots d_{\left(N_{\mathrm{c}}-1\right) l_{p}}\left(\phi_{l p}, \theta_{l p}\right)\right)^{\mathrm{T}}$.

The receiving process of the proposed MIMO receiver with APM is illustrated in Figure 2. We consider that the number of transmit antennas at the transmitter is $N_{\mathrm{t}}$. Suppose that the channel coefficient is constant during a transmitted symbol. In addition, we assume that the signals transmitted from $N_{\mathrm{t}}$ antennas suffer independent fading. Also, the transmitter is assumed to have no channel state information. Thus, the average transmit power of each transmitted symbol is assumed to be equivalent to each other. When we show the transmitted symbol from the $l$-th transmit antenna as $s_{l}$ (Figure 2), then, we can have $\mathrm{E}\left[\left|s_{l}\right|^{2}\right]=1$ for $l=1, \ldots, N_{\mathrm{t}}$ without loss of generality. Besides, the symbol is assumed to be an independent and identically distributed (i.i.d.) random variable. The number of arrival paths per transmit antenna is $N_{\mathrm{p}}$.

\footnotetext{
${ }^{2}$ In particular, in the case of the ESPAR antenna, the conversions from the applied voltage to the reactance and from the reactance to the antenna pattern could be nonlinear. Then, the assumption might be optimistic in reality. However, in some cases, we have shown for the conversion from the reactance to the antenna pattern that the effect of the nonlinearity can be suppressed by considering the conversion characteristics $[15,16]$.
} 


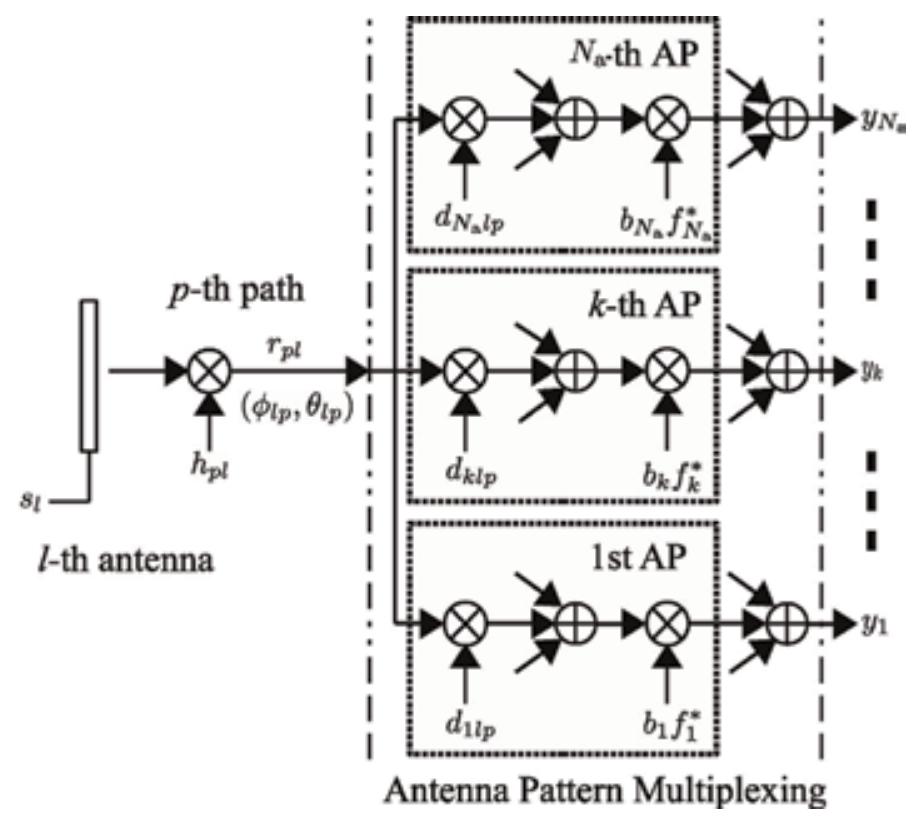

Figure 2.

Receiving process of the receiver with APM.

The $p$-th path sent from $l$-th transmit antenna received at the direction of $\left(\phi_{l p}, \theta_{l p}\right)$ is given as follows:

$$
r_{p l}=h_{p l} \cdot s_{l}
$$

where $h_{p l}$ means a channel coefficient of a link between the $l$-th transmit antenna and $p$-th direction for the antenna and is a complex Gaussian random variable with zero mean and variance of unity.

Now we consider the received signals from $N_{\mathrm{t}}$ transmit antennas. Since $N_{\mathrm{p}}$ paths per transmit antenna arrive at the receiver, the output from the antenna with APM can be shown as follows:

$$
\begin{aligned}
x(t) & =\sum_{l=1}^{N_{\mathrm{t}}} \sum_{p=1}^{N_{\mathrm{p}}} d_{l p}\left(\phi_{l p}, \theta_{l p}, t\right) \cdot r_{p l}+n(t) \\
& =\sum_{l=1}^{N_{\mathrm{t}}} \sum_{p=1}^{N_{\mathrm{p}}} \sum_{k=1}^{N_{\mathrm{a}}} d_{k l p}\left(\phi_{l p}, \theta_{l p}\right) \cdot b_{k} \cdot f_{k}(t) \cdot h_{p l} \cdot s_{l}+n(t)
\end{aligned}
$$

where $n(t)$ is an additive white Gaussian noise (AWGN) component. As shown in Figure 2, the received signal $r_{p l}$ is multiplied by $N_{\mathrm{a}}$ multiplexed antenna patterns $d_{k l p}\left(\phi_{l p}, \theta_{l p}\right)$. Since $N_{\mathrm{p}}$ paths are transmitted from the transmit antenna and antenna patterns are orthogonal to each other, the received components for $N_{\mathrm{p}}$ paths are added in each antenna pattern domain separately.

Replacing $x(t)$ with the corresponding vector $\mathbf{x}$, we have the received signal in matrix form as

$$
\boldsymbol{x}=\sum_{l=1}^{N_{\mathrm{t}}} \sum_{p=1}^{N_{\mathrm{p}}} C B \boldsymbol{d}_{l p} h_{p l} s_{l}+\boldsymbol{n}
$$




$$
=\sum_{l=1}^{N_{\mathrm{t}}} C B D_{l} \boldsymbol{h}_{l} s_{l}+\boldsymbol{n}
$$

where $D_{l}$ is an antenna pattern matrix for $l$-th transmitted symbol whose size is $N_{\mathrm{a}} \times N_{\mathrm{p}}$ and is given as

$$
D_{l}=\left(\begin{array}{llll}
\boldsymbol{d}_{l 1} & \boldsymbol{d}_{l 2} & \ldots & \boldsymbol{d}_{l N_{\mathrm{p}}}
\end{array}\right)
$$

and $\boldsymbol{h}_{l}$ shows a channel vector whose length is $N_{\mathrm{p}}$ and can be given as

$$
\boldsymbol{h}_{l}=\left(\begin{array}{llll}
h_{1 l} & h_{2 l} & \ldots & h_{N_{\mathrm{p}} l}
\end{array}\right)^{\mathrm{T}},
$$

and $\boldsymbol{n}$ is a noise vector whose length is $N_{\mathrm{c}}$ and whose element $n_{k}$ is an i.i.d. white Gaussian random variable with zero mean and variance $\sigma_{n}^{2} / N_{\mathrm{c}}$. Then, the autocorrelation matrix of $\boldsymbol{n}$ can be defined as follows:

$$
\mathrm{E}\left[\boldsymbol{n} \boldsymbol{n}^{\mathrm{H}}\right]=\frac{\sigma_{n}^{2}}{N_{\mathrm{c}}} I_{N_{\mathrm{c}}}
$$

Eq. (18) can be further simplified as

$$
\boldsymbol{x}=C B D H \boldsymbol{s}+\boldsymbol{n},
$$

by introducing the antenna pattern matrix $D$ defined as

$$
D=\left(\begin{array}{llll}
D_{1} & D_{2} & \ldots & D_{N_{\mathrm{t}}}
\end{array}\right),
$$

and the channel matrix $H$, which is a block matrix of $\boldsymbol{h}_{l}$, defined as

$$
H=\left(\begin{array}{cccc}
\boldsymbol{h}_{1} & \mathbf{0} & \ldots & \mathbf{0} \\
\mathbf{0} & \boldsymbol{h}_{2} & \ddots & \vdots \\
\vdots & \ddots & \ddots & \mathbf{0} \\
\mathbf{0} & \ldots & \mathbf{0} & \boldsymbol{h}_{N_{\mathrm{t}}}
\end{array}\right)
$$

where $\mathbf{0}$ is a zero and column vector of length $N_{\mathrm{p}}$ and $s$ is a vector of transmitted symbols defined as

$$
\boldsymbol{s}=\left(\begin{array}{llll}
s_{1} & s_{2} & \ldots & s_{N_{\mathrm{t}}}
\end{array}\right)^{\mathrm{T}},
$$

and its autocorrelation function is given as follows from the assumption:

$$
\mathrm{E}\left[\boldsymbol{s s}^{\mathrm{H}}\right]=I_{N_{\mathrm{t}}}
$$

The output signal $\boldsymbol{x}$ of the antenna is multiplied by the complex conjugate of the applied waveform. This signal process can be achieved by multiplying $B^{-1} C^{\mathrm{H}}$ by $\boldsymbol{x}$ from the left-hand side, that is, from Eq. (22) to Eq. (11) as

$$
\begin{gathered}
\boldsymbol{y}=B^{-1} C^{\mathrm{H}} \boldsymbol{x} \\
=B^{-1} C^{\mathrm{H}}(C B D H \boldsymbol{s}+\boldsymbol{n})
\end{gathered}
$$




$$
\begin{gathered}
=B^{-1} C^{\mathrm{H}} C B D H \mathbf{s}+B^{-1} C^{\mathrm{H}} \mathbf{n} \\
=D H \boldsymbol{s}+\boldsymbol{n}^{\prime}
\end{gathered}
$$

where $\boldsymbol{n}^{\prime}=B^{-1} C^{\mathrm{H}} \boldsymbol{n}$. The autocorrelation matrix of $\boldsymbol{n}^{\prime}$ can be derived as follows;

$$
\begin{gathered}
\mathrm{E}\left[\boldsymbol{n}^{\prime} \boldsymbol{n}^{\prime \mathrm{H}}\right]=\mathrm{E}\left[B^{-1} C^{\mathrm{H}} \boldsymbol{n}\left(B^{-1} C^{\mathrm{H}} \boldsymbol{n}\right)^{\mathrm{H}}\right] \\
=\mathrm{E}\left[B^{-1} C^{\mathrm{H}} \boldsymbol{n} \boldsymbol{n}^{\mathrm{H}} C B^{-1 \mathrm{H}}\right]
\end{gathered}
$$

Here, since the code set $C$ and the matrix $B$ are fixed, and from Eq. (21), we have

$$
\begin{aligned}
& \mathrm{E}\left[\boldsymbol{n}^{\prime} \boldsymbol{n}^{\prime \mathrm{H}}\right]=B^{-1} C^{\mathrm{H}} \mathrm{E}\left[\boldsymbol{n} \boldsymbol{n}^{\mathrm{H}}\right] C B^{-1 \mathrm{H}} \\
& =\frac{\sigma_{n}^{2}}{N_{\mathrm{c}}} B^{-1} C^{\mathrm{H}} C B^{-1 \mathrm{H}} \\
& =\frac{\sigma_{n}^{2}}{N_{\mathrm{c}}} B^{-1} B^{-1 \mathrm{H}}
\end{aligned}
$$

where $B^{-1}$ is a diagonal matrix because $B$ is a diagonal matrix. If we use $b_{k}$ whose absolute value is unity as $\left|b_{k}\right|=1$, the $k$-th diagonal element of $B^{-1}$ is $b_{k}^{*}$. Therefore, we can derive the relation $B^{-1 \mathrm{H}}=B$. With the relation between $B^{-1 \mathrm{H}}$ and $B$, we can modify Eq. (35) as

$$
\begin{gathered}
\mathrm{E}\left[\boldsymbol{n}^{\prime} \boldsymbol{n}^{\prime \mathrm{H}}\right] \\
=\frac{\sigma_{n}^{2}}{N_{\mathrm{c}}} B^{-1} B^{-1 \mathrm{H}} \\
=\frac{\sigma_{n}^{2}}{N_{\mathrm{c}}} B^{-1} B \\
=\frac{\sigma_{n}^{2}}{N_{\mathrm{c}}} I_{N_{\mathrm{c}}} .
\end{gathered}
$$

Thus, the autocorrelation matrix of $\boldsymbol{n}^{\prime}$ is equivalent to that of $\boldsymbol{n}$.

The process of Eq. (27) can be implemented by multiplying $b_{k} f_{k}(t)$ by $x(t)$ in parallel and integrating them over the interval $T_{\mathrm{s}}$ or with a correlator as shown in Figure 2. Since $N_{\mathrm{t}}$ transmit antennas are assumed, $N_{\mathrm{t}}$ components are added in each antenna pattern domain. Note that the process divides a single signal output into $N_{\mathrm{a}}$ outputs or $N_{\mathrm{a}}$ antenna pattern domains.

If we recognise the matrix $D H$ in Eq. (30) as an equivalent channel matrix $G=D H$ that is equivalent to that of the conventional MIMO systems, we can rewrite Eq. (30) as

$$
\boldsymbol{y}=G \boldsymbol{s}+\boldsymbol{n}^{\prime}
$$

Since the length of $\boldsymbol{y}$ is $N_{\mathrm{a}}$, the proposed MIMO with APM seems equivalent to conventional $N_{\mathrm{t}} \times N_{\mathrm{a}}$ MIMO systems [19]. The number $N_{\mathrm{a}}$ shows the number of orthogonal antenna patterns in time domain ${ }^{3}$. However, the number corresponds to the number of virtual receive antennas in the context of MIMO receivers. The

\footnotetext{
3 The orthogonality in time domain does not guarantee the orthogonality in space domain or in terms of directivity. It is a challenging problem to develop a set of orthogonal functions in both time and space domains.
} 
equation above realises that the received components obtained by the receiver with APM are similar to those of the conventional MIMO systems. We assume that the receiver has perfect knowledge of the equivalent channel matrix $G$. Note that the receiver does not need to know every element of $D$ or $H$ for decoding. In practice, it may even be impossible to separately evaluate the components of $D$ and $H$.

\subsection{Capacity of MIMO systems with APM}

From the received signal in Eq. (39) and the autocorrelation matrix of the transmitted symbols in Eq. (26), we can derive the ergodic capacity $C^{4}$ as

$$
C=\mathrm{E}\left[\log _{2} \operatorname{det}\left(I_{N_{\mathrm{t}}}+\frac{\gamma}{N_{\mathrm{t}}} G^{\mathrm{H}} G\right)\right]
$$

where det is the determinant of a matrix and $\gamma$ is the average signal-to-noise ratio (SNR) per transmit antenna and is defined as $\gamma=1 / \sigma_{n}^{2}$. As we can see from

Eq. (40), the capacity depends on the property of the equivalent channel matrix $G$ or $D H$. Here, the matrix $G$ satisfies the following properties which are similar to the channel matrix of the conventional MIMO systems:

$$
\begin{gathered}
\mathrm{E}\left[G G^{\mathrm{H}}\right]=N_{\mathrm{t}} I_{N_{\mathrm{a}}}, \\
\mathrm{E}\left[G^{\mathrm{H}} G\right]=N_{\mathrm{a}} I_{N_{\mathrm{t}}}
\end{gathered}
$$

\section{Numerical results}

In this section, we show the ergodic capacity of a MIMO system whose receiver uses the proposed APM technique. Through the section the number of transmit antennas is $N_{\mathrm{t}}=2$. The capacity $C$ (40) of the proposed MIMO system is shown in Figure 3. We assume that the coefficients of the matrix $D$ are denoted as $\exp (j \Theta) / \sqrt{N_{\mathrm{p}}}$ where $\Theta$ is a uniform random variable in the interval $[0,2 \pi)$. In the figure, the number of antenna patterns or virtual antenna outputs $N_{\mathrm{a}}=8$. If the channel coefficient is constant while a symbol is transmitted, the code set satisfying Eq. (11) could not affect on the performance. Thus, we do not specify the code set in this study. We evaluate the capacities for the number of arrival paths $N_{\mathrm{p}}=1,2,4$, 8,16 , and 32 and show them with solid lines. For comparison, we also show the ergodic capacity of the conventional MIMO systems with black dashed lines marked with dots (' $\cdot$ '). The pairs of transmit and receive antennas are $2 \times 1 \mathrm{MIMO}$ and $2 \times 2$ MIMO systems.

The capacities of the MIMO systems with APM are between those of the conventional $2 \times 1$ and $2 \times 2$ MIMO systems. In lower SNR region, the capacity of MIMO with APM for various $N_{\mathrm{p}}$ is close to that of the conventional $2 \times 1 \mathrm{MIMO}$. On the other hand, in higher SNR region, the values and also the slopes of the capacities converge to those of the conventional $2 \times 2 \mathrm{MIMO}$. When the number of arrival paths $N_{\mathrm{p}}$ increases, the capacities also increased and converged towards the capacity of the conventional $2 \times 2$ MIMO system. Since the slope of the capacity relates to diversity order, the proposed APM technique can achieve the same order as the conventional $2 \times 2 \mathrm{MIMO}$ systems even if $N_{\mathrm{p}}=1$. Because of the increased

\footnotetext{
${ }^{4}$ We use the same variable character as code matrix. Since they are used in different contexts, they might be easily distinguishable.
} 




Figure 3.

Ergodic capacity of MIMO systems with APM technique versus average SNR for various number of arrival paths $N_{p}$. $\left(N_{t}=2, N_{a}=8\right)$.

capacity due to the increase in the number of arrival paths, it can be recognised that the proposed APM obtains path diversity gain. As mentioned in Section 2, APM technique can reduce the antenna size and hardware cost. Thus, we can find that the proposed technique can provide similar capacities with reduced size and less hardware cost.

We show the ergodic capacities versus average SNR of the proposed MIMO systems with APM for fixed number of arrival paths $N_{\mathrm{p}}=16$ and various number of antenna patterns, i.e., $N_{\mathrm{a}}=1,2,4,8$, and 16, in Figure 4. The number $N_{\mathrm{p}}=16$ might be sufficiently large to obtain the path diversity gain according to Figure 3 . For comparison purposes, the performances of the conventional $2 \times 1$ and $2 \times 2$ MIMO systems are also drawn.

The capacities for APM techniques increase in the number of antenna patterns $N_{\mathrm{a}}$ and converge to those of the conventional $2 \times 1$ MIMO systems in lower SNR region and the $2 \times 2 \mathrm{MIMO}$ systems in higher SNR region. When $N_{\mathrm{a}}=16$, the capacity almost overlaps the capacity of the conventional $2 \times 2$ MIMO systems in the region average SNR, which is more than $20 \mathrm{~dB}$. In the case $N_{\mathrm{a}}=1$, the capacity

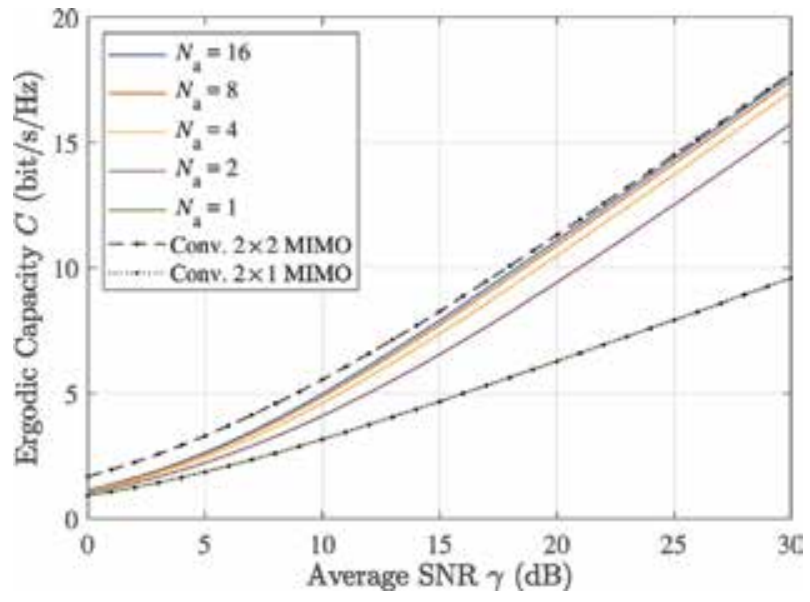

Figure 4 .

Ergodic capacity of MIMO systems using receiver with APM technique for various number of orthogonal antenna patterns. Antenna pattern matrix has constant amplitude and random phase with uniform distribution. $\left(N_{t}=2, N_{p}=16\right)$. 


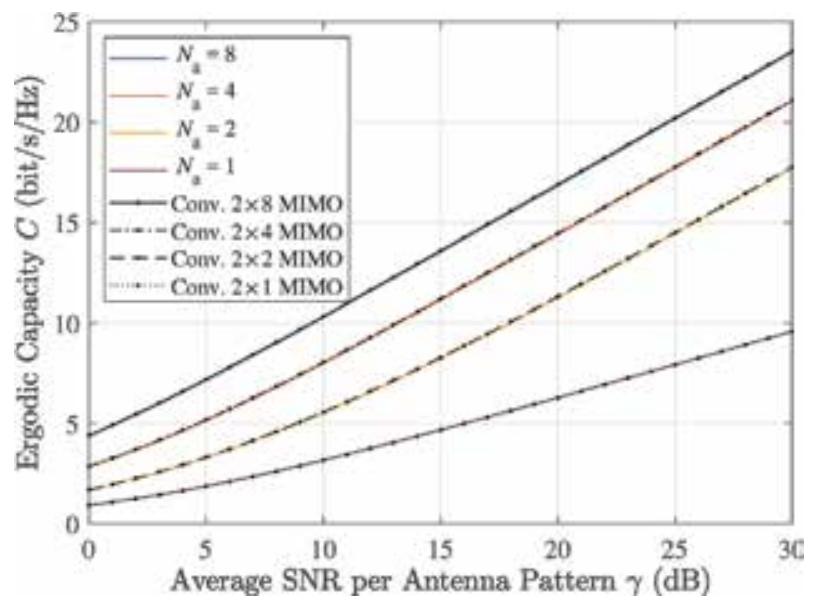

Figure 5 .

Ergodic capacity of MIMO systems with APM technique for various number of orthogonal antenna patterns. Average SNR per antenna pattern is a constant $\left(N_{t}=2, N_{p}=16\right)$.

of the proposed MIMO systems with APM is equivalent to that of the conventional $2 \times 1$ MIMO system. Hence, diversity gain cannot be obtained even if the number of arrival paths is sufficiently large.

Then we consider the case that the average SNR per antenna pattern or virtual antenna is given as $\gamma$. In this case, the coefficients in the matrix $D$ are random variables shown as $\exp (j \Theta)$ where $\Theta$ is a uniform random variable in the interval $[0,2 \pi)$. The ergodic capacities of the proposed MIMO systems with APM are shown for $N_{\mathrm{a}}=1,2,4$, and 8 for $N_{\mathrm{p}}=16$ in Figure 5. In the figure, we also illustrate the capacities of the conventional MIMO systems for $2 \times 1,2 \times 2,2 \times 4$, and $2 \times 8$ in terms of the numbers of transmit and receive antennas. As we can see from the figure, the capacities are equivalent to each other between $N_{\mathrm{a}}$ for APM and the same number of receive antennas for the conventional MIMO. For example, when $N_{\mathrm{a}}=8$, the capacity is the same as that of $2 \times 8 \mathrm{MIMO}$ systems. Therefore, when the average SNR per antenna pattern is same as the average SNR per the number of receive antennas, the proposed APM-based MIMO systems with $N_{\mathrm{a}}$ antenna patterns achieve almost equivalent capacity to the conventional $N_{\mathrm{t}} \times N_{\mathrm{a}} \mathrm{MIMO}$ systems.

\section{Conclusions}

In this chapter we propose a concept of APM for MIMO receiver to reduce the antenna size and hardware cost with keeping the availability of diversity gain. We discuss the types of antennas which achieve the APM, i.e., generating time-varying antenna pattern. Also, we discuss the benefits of the antennas, in particular, for ESPAR antenna-based structure. The number of virtual antennas or antenna patterns can be increased with the number of multiplexed orthogonal signals used to change the antenna patterns. A model of receiving process is proposed for analysing the capacity of systems using APM. We derive a model of received signals to analyse the system performance. The received signal in matrix form includes an equivalent channel matrix, which is a product of antenna pattern matrix, the channel coefficient vector for each output.

When the number of arrival paths and the number of antenna pattern are sufficiently large, the ergodic capacity approaches to that of $2 \times 2$ MIMO systems. 
The property deduces the proposed APM, which can obtain diversity gain from path diversity and diversity reception based on the virtual antennas.

On the other hand, numerical results show that the ergodic capacity is equivalent to that of the conventional MIMO systems when the average SNR per antenna pattern is constant. Then, the proposed APM-based receiver can exploit path diversity gain and antenna pattern diversity maximally without additional physical antenna elements.

Future work is a development of efficient multiplexed antenna patterns, which have larger number of orthogonal antenna patterns than the number of antenna elements equipped with a cable.

\section{Acknowledgements}

This work was carried out by the joint usage/research programme of the Institute of Materials and Systems for Sustainability (IMaSS), Nagoya University.

\section{Abbreviations}

$\begin{array}{ll}\text { AP } & \text { antenna pattern } \\ \text { APM } & \text { antenna pattern multiplexing } \\ \text { CDM } & \text { code-division multiplexing } \\ \text { CDMA } & \begin{array}{l}\text { code-division multiple access } \\ \text { DS/SS }\end{array} \\ \text { direct-sequence spread spectrum } \\ \text { ESPAR } & \text { electronically steerable passive array radiator } \\ \text { i.i.d. } & \text { independent and identically distributed } \\ \text { MIMO } & \text { multiple-input multiple-output } \\ \text { MISO } & \text { multiple-input single-output } \\ \text { MSE } & \text { modulated scattering element } \\ \text { OFDM } & \text { orthogonal frequency-division multiplexing } \\ \text { PSK } & \text { phase-shift keying } \\ \text { QAM } & \text { quadrature amplitude modulation } \\ \text { RF } & \text { radio frequency } \\ \text { SIMO } & \text { single-input multiple-output } \\ \text { SINR } & \text { signal-to-interference-plus-noise ratio } \\ \text { SISO } & \text { single-input single-output } \\ \text { SNR } & \text { signal-to-noise ratio } \\ \text { VRE } & \text { variable reactance element }\end{array}$




\section{Author details}

Masato Saito

Faculty of Engineering, University of the Ryukyus, Nishihara, Japan

*Address all correspondence to: massai@ieee.org

\section{IntechOpen}

(C) 2019 The Author(s). Licensee IntechOpen. This chapter is distributed under the terms of the Creative Commons Attribution License (http://creativecommons.org/licenses/ by/3.0), which permits unrestricted use, distribution, and reproduction in any medium, provided the original work is properly cited. (c) BY 


\section{References}

[1] Ohira T, Gyoda K. Electronically steerable passive array radiator antennas for low-cost analog adaptive beamforming. In: Proceedings 2000 IEEE International Conference on Phased Array Systems and Technology (Cat. No.00TH8510). 2000. pp. 101-104. DOI: 10.1109/ PAST.2000.858918

[2] Chen Q, Takeda Y, Yuan Q, Sawaya K. Diversity performance of modulated scattering array antenna. IEICE Electronics Express. 2007;4(7): 216-220. DOI: $10.1587 /$ elex.4.216

[3] Chen Q, Wang L, Iwaki T, Kakinuma Y, Yuan Q, Sawaya K. Modulated scattering array antenna for MIMO applications. IEICE Electronics Express. 2007;4(23):745-749. DOI: 10.1587/elex.4.745

[4] Wang L, Chen Q, Yuan Q, Sawaya K. Diversity performance of modulated scattering antenna array with switched reflector. IEICE Electronics Express. 2010;7(10):728-731. DOI: $10.1587 /$ elex.7.728

[5] Wang L, Chen Q, Yuan Q, Sawaya K. Numerical analysis on MIMO performance of the modulated scattering antenna array in indoor environment. IEICE Transactions on Communications. 2011;E94-B: 1752-1756. DOI: 10.1587/transcom.E94. B.1752

[6] Yuan Q, Ishizu M, Chen Q, Sawaya K. Modulated scattering array antenna for mobile handset. IEICE Electronics Express. 2005;2(20): 519-522. DOI: $10.1587 /$ elex.4.745

[7] Bains R, Muller R. Using parasitic elements for implementing the rotating antenna for mimo receivers. IEEE Transactions on Wireless Communications. 2008;7:4522-4533. DOI: 10.1109/T-WC.2008.060808
[8] Kalis A, Kanatas AG, Papadias CB, editors. Parasitic Antenna Arrays for Wireless MIMO Systems. New York: Springer-Verlag; 2014. DOI: 10.1007/ 978-1-4614-7999-4

[9] Reinoso Chisaguano DJ, Hou Y, Higashino T, Okada M. Low-complexity channel estimation and detection for mimo-ofdm receiver with espar antenna. IEEE Transactions on Vehicular Technology. 2016;65: 8297-8308. DOI: $10.1109 /$ TVT.2015.2506782

[10] Arita W, Saito M. Novel receive diversity scheme using espar antenna and arbitrary frequency band. In: 2012 IEEE Vehicular Technology Conference (VTC Fall). 2012. pp. 1-5. DOI: 10.1109/ VTCFall.2012.6399283

[11] Dia A, Saito M. A study on channel capacity of mimo systems with a receiver with antenna pattern modulation. In: 2017 International Symposium on Antennas and Propagation (ISAP). 2017. DOI: 10.1109/ISANP.2017.8228953

[12] Idoguchi Y, Saito M. Evaluation of antenna with periodically variable directivity. In: 2014 Asia-Pacific Microwave Conference. 2014. pp. 345-347

[13] Idoguchi Y, Saito M. A study on received signal spectrum of antenna with periodically variable directivity. In: 2014 International Symposium on Antennas and Propagation Conference Proceedings. 2014. pp. 403-404

[14] Saito M. A study on mimo systems with antenna pattern modulation technique. In: IEICE Tech. Rep. 2017. pp. 51-56. (in Japanese)

[15] Kawano K, Idoguchi Y, Saito M. Evaluation of power spectrum of 2-element dipole antenna with 
periodically variable antenna pattern. In: 2016 International Symposium on Antennas and Propagation (ISAP). 2016. pp. 412-413

[16] Kawano K, Saito M. Periodic reactance time functions for 2-element espar antennas applied to 2-output simo/mimo receivers. IEICE Transactions on Communications. 2019; E102.B(4):930-939. DOI: 10.1587/ transcom.2018EBP3097

[17] Saito M. Periodically variable antenna pattern for maximizing path diversity gain in mimo receivers. In: 2018 5th IEEE Uttar Pradesh Section International Conference on Electrical, Electronics and Computer Engineering (UPCON). 2018. pp. 1-6. DOI: 10.1109/ UPCON.2018.8597063

[18] Sun C, Hirata A, Ohira T, Karmakar NC. Fast beamforming of electronically steerable parasitic array radiator antennas: Theory and experiment. IEEE Transactions on Antennas and Propagation. 2004;52(7): 1819-1832. DOI: $10.1109 /$

TAP.2004.831314

[19] Proakis JG, Salehi M. Digital Communications. 5th ed. Boston: McGraw-Hill Education; 2007 


\title{
Chapter 5
}

\section{Broadside Pattern Correction Techniques for Conformal Antenna Arrays}

\author{
Irfan Ullah, Shahid Khattak and Benjamin D. Braaten
}

\begin{abstract}
Phase compensation techniques based on projection method and convex optimization (phase correction only) for comparing the maximum gain of a phase-compensated conformal antenna array have been discussed. In particular, these techniques are validated with conformal phased array antenna attached to a cylindrical-shaped surface with various radii of curvatures. These phase compensation techniques are used to correct the broadside radiation pattern. It is shown that the maximum broadside gain compensated is still less than the gain of a linear flat array for any surface deformation. This fundamental maximum compensated gain limitations of the phase compensation techniques can be used by a designer to predict the maximum broadside obtainable theoretical gain on a conformal antenna array for a particular deformed surface.
\end{abstract}

Keywords: conformal antennas, phased arrays, antenna radiation patterns, convex optimization, microstrip arrays

\section{Introduction}

Conformal antenna arrays are beneficial for applications that need an antenna to be placed on a non-flat surface, for example, on the fuselage of a UAV/airplane in the aerospace industry [1-3], implantable sensors in wearable networks [4-8], and satellite communications [9-11]. One of the main advantages of using conformal antenna is its structural integration ability on singly curved (e.g., a wedge/cylinder) [12-15] and doubly curved (like a sphere) surfaces [16]. This can be very useful in applications where using definite flat surface may not be a practical design choice. Another exciting application of conformal antenna array is at the base station in a cellular mobile communication system. Today, mobile service providers are utilizing three separate antenna panels (dipole or monopole array) in a cell for a $120^{\circ}$ sector coverage. What about, if one cylindrical array is used instead of three dipole arrays [17]? This can result in a much smaller transmitter requirement with $360^{\circ}$ azimuthal coverage plus reduced base station size at a lower cost (specifically beneficial in crowded residential areas where cellular companies have to rent the space for base station installation).

On the other hand, these curved surfaces may be subjected to intentional (e.g., flexing wings of a UAV/aircraft) and/or unintentional (bending of aircraft wings due to severe weather conditions/vibrations) forces that change the shape of the 


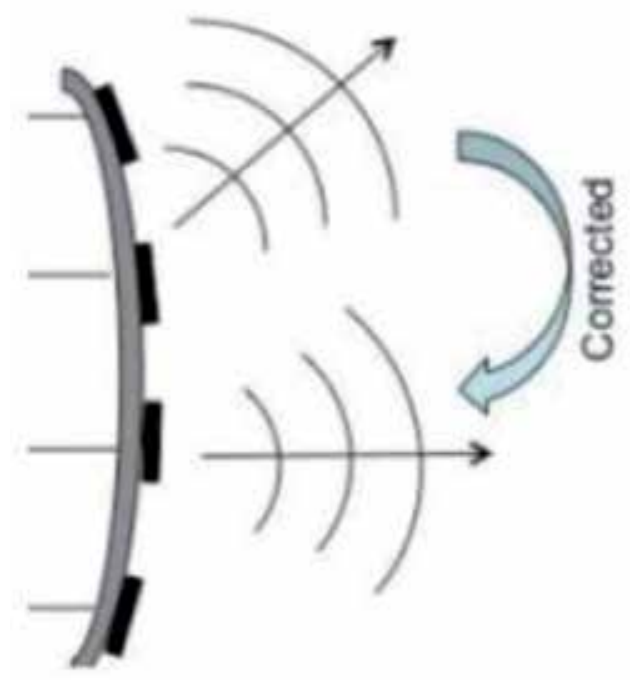

Figure 1.

Array on a conformal surface.

surface [12]. As a result, the radiation pattern of the conformal antenna array is changed as shown in Figure 1. The results in [18] indicate that directivity of conformal antenna array can be reduced by $5-15 \mathrm{~dB}$. In the literature, various methods have been proposed to compensate the reduction in directivity and to improve/correct the radiation pattern of a conformal antenna array. In [1-3, 11, 19-21], mechanical calibration techniques have been used to steer the main beam on a conformal surface in the desired direction. In $[12,13,15,16,22-25]$, projection method of [26] is used to correct the main beam direction of a deformed/flex surface. In [27-30], various optimization algorithms have been used to control the radiation pattern of conformal antenna arrays. In summary, it has been shown that the radiation pattern of a conformal antenna array can be improved with different calibration techniques, signal processing algorithms, sensor circuitry, and phase and amplitude adjustments.

This chapter will focus on phase compensation of four-element conformal cylindrical antenna array using (1) projection method and (2) convex optimization method. First, a brief introduction and working principle of phase compensation is presented using projection method. Then, array factor expression will be derived to compensate the radiation pattern of conformal cylindrical array. Then, the convex optimization algorithm will be discussed to compute the array weights for pattern recovery of conformal cylindrical array. Then, compensated gain using both the methods will be compared to linear flat array to explore the gain limitations of these compensated techniques for conformal antenna arrays. Finally, conclusion and future work are presented.

\section{Projection method for pattern recovery of conformal antenna array}

The projection method in [26] and its further exploration in [12, 15, 22] are adopted here to describe the behavior of the conformal antenna array shown in Figure 2. For discussion, consider the problem where the flat antenna array is placed on the singly curved surface shaped as a cylinder with radius $r$ as shown in Figure 2. The position of each antenna element on the cylinder is represented 


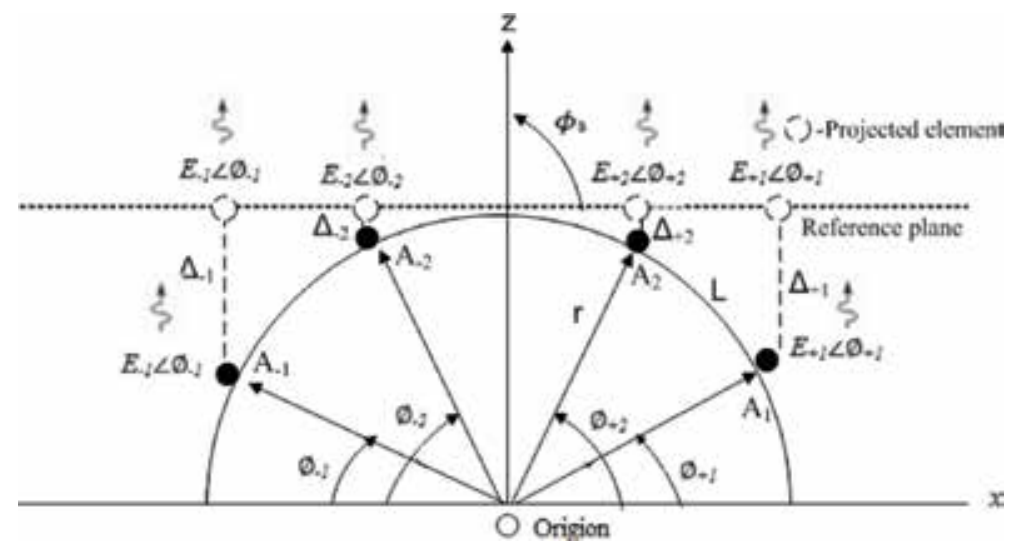

Figure 2.

Phase compensation of a conformal cylindrical antenna array.

as $A_{ \pm \mathrm{n}}, n=1,2$ (for four antenna elements on the cylindrical surface). If each antenna element on the cylindrical surface is excited with uniform amplitudes and phases, that is, if excitation weights $w_{n}=1 e^{j 0}$ put on all elements, then the E-fields radiated from the antenna elements will arrive at the reference plane with different phases. This phase difference is due to the different path lengths experienced by the radiated E-fields in the $\mathrm{z}$ direction while reaching to the reference plane. Because phases of E-fields radiated from $A_{ \pm 1}$ are not the same as those radiated by $A_{ \pm 2}$, the radiation may not necessarily be constructively added broadside to the array (i.e., with $\phi_{s}=90$ ). If the E-fields $E_{ \pm n} e^{j \phi_{ \pm n}}$ from antenna elements are ensured to arrive at the reference plane with the same phase, then constructive interference will result in a broadside radiation pattern and therefore pattern recovery toward the broadside is possible. The delayed phase of E-fields due to free-space propagation $\left(-k \Delta_{ \pm n}\right)$ from antenna elements on the conformal surface to the reference plane can be compensated (corrected) by exciting the elements with phase advance $\left(+k \Delta_{ \pm n}\right)$. This will cause the E-fields from antenna elements to arrive at the reference plane with same phase and will cause constructive broadside radiation pattern in the $+\mathrm{z}$ direction.

To compute this compensated phase, the antenna elements are projected on the reference plane and then the distances from antenna elements on cylindrical surface (shown as black dots) to the projected elements on the reference plane (shown as dashed circles) are calculated. Suppose $\Delta_{-1}, \Delta_{-2}, \Delta_{2}$, and $\Delta_{1}$ denote the distances from the elements $A_{-1}, A_{-2}, A_{2}$, and $A_{1}$, respectively, to the reference plane (or the projected elements on the reference plane).

\subsection{Computing the distance to the projected elements}

The distance from the antenna elements on the cylinder to the projected elements on the reference plane can be computed using:

$$
\Delta_{ \pm \mathrm{n}}=r-r \sin \phi_{ \pm \mathrm{n}}
$$

where $\mathbf{r}$ is the radius of the cylinder and $\phi_{ \pm n}$ is the angular position of antenna elements on the cylinder. Using the relation of $\mathbf{L}=\mathbf{r} \boldsymbol{\theta}$, the angular positions of antenna elements can be calculated, where $L=\lambda / 2$ is the inter-element distance on the cylindrical surface. 


\subsection{Computing the compensated phase}

The required compensated phase to correct the broadside radiation pattern of a conformal cylindrical antenna array in Figure 2 is then given by:

$$
\delta_{ \pm \mathrm{n}}^{c}=+k \Delta_{ \pm \mathrm{n}}
$$

where $k$ is the free-space wave number.

\subsection{Array factor expression}

To analytically compute the corrected (compensated) radiation pattern and validate with simulation results, the following compensated array factor $\mathrm{AF}_{c}$ is used $[18,31]$ :

$$
\mathrm{AF}_{c}=\mathrm{AF} e^{j \delta_{ \pm n}{ }^{c}}
$$

where $\delta_{ \pm n}{ }^{c}$ can be computed using Eq. (2) and the array factor (AF) expression for antennas on conformal surfaces is [18]:

$$
\mathrm{AF}=\sum_{n=1}^{N} F_{n}(\theta, \phi) w_{n} e^{j k\left[x_{n} \sin \theta+z_{n} \cos \theta\right]},
$$

where $k$ is the free space wave number, $N$ is number of antenna elements, $\left(x_{n}, z_{n}\right)$ is the location of nth antenna element on the conformal surface, and $F_{n}(\theta, \phi)$ is the individual element pattern in Figure 2. $w_{n}=I_{n} e^{j \Delta \phi}$ is the complex weighting function required to drive the nth antenna element.

For this work, the phase difference $\Delta \phi$ between adjacent antenna elements was made zero, and the amplitude tapering coefficient $I_{n}$ was kept equal to 1 (uniform excitation). Using Eqs. (1)-(4), the expression for corrected array factor is given by:

$$
\begin{aligned}
\mathrm{AF}_{c} & =F_{1}(\theta, \phi) e^{j k \Delta_{-1}} e^{j k\left[x_{1} \sin \theta+z_{1} \cos \theta\right]}+F_{2}(\theta, \phi) e^{j k \Delta_{-2}} e^{j k\left[x_{2} \sin \theta+z_{2} \cos \theta\right]} \\
& +F_{3}(\theta, \phi) e^{j k \Delta_{+2}} e^{j k\left[x_{3} \sin \theta+z_{3} \cos \theta\right]}+F_{4}(\theta, \phi) e^{j k \Delta_{+1}} e^{j k\left[x_{4} \sin \theta+z_{4} \cos \theta\right]}
\end{aligned}
$$

$F_{1}(\theta, \phi)=\cos \left(\theta+\phi_{-1}\right)$ and $F_{2}(\theta, \phi)=\cos \left(\theta+\phi_{-2}\right)$ are the element patterns for the two left antenna elements and $F_{3}(\theta, \phi)=\cos \left(\theta-\phi_{+1}\right)$ and $F_{4}(\theta, \phi)=\cos \left(\theta-\phi_{+2}\right)$ are the element patterns for the two right antenna elements on the cylindrical surface in Figure 2. It should be noted that for linear array, the element pattern is $\cos (\theta)$ as all the elements are pointing towards zenith (that is towards $\phi_{s}=0^{\circ}$ ). However on conformal surfaces, the individual element patterns become geometry dependent. For example, as can be seen in Figure 2, the look directions of antenna elements $A_{-1}$ and $A_{-2}$ are towards the angular directions $\phi_{-1}$ and $\phi_{-2}$ respectively, while the look directions of antenna elements $A_{1}$ and $A_{2}$ are towards the angular directions $\phi_{1}$ and $\phi_{2}$ respectively. Therefore, the element patterns are $\cos \left(\theta+\phi_{-1}\right), \cos \left(\theta+\phi_{-2}\right), \cos$ $\left(\theta-\phi_{1}\right)$, and $\cos \left(\theta-\phi_{2}\right)$ respectively.

\section{Convex optimization for pattern recovery of conformal antenna array}

The broadside gain maximization problem of a conformal antenna array in Figure 2 can be formulated as a linear constrained quadratic programming problem [32], i.e.,

Subject to

$$
\text { Minimize }\left\|\mathbf{w}_{\mathbf{n}}\right\| \text {, }
$$

$$
\mathrm{AF}_{\text {target }}=1 \text {, }
$$




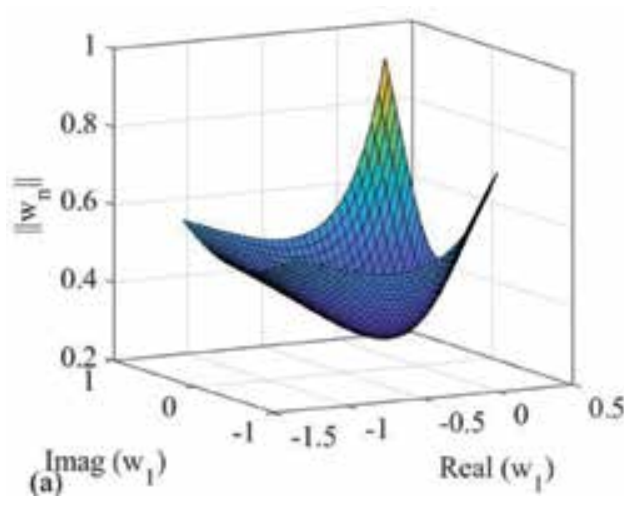

Figure 3.

(a) $3 D$ plot of the objective function plotted as a function of real ( $\left.\begin{array}{l}w 1 \\ 1\end{array}\right)$ and imaginary $(w 1)$. (b) the contour plot of the objective function with illustration of gradient descent method.

where $\mathbf{w}_{\mathbf{n}} \in \mathbb{C}^{[N \times 1]}$ is the complex weighing vector, which gives the objective function $2 N$ degrees of freedom. In Figure 3(a) the objective function plotted for the two component dimensions of the first antenna weight $w_{1}$ clearly indicates its convex form in the vicinity of the origin. The contour plots in the considered two dimensions are also presented in Figure 3(b). The aspect of the contour lines along these two dimensions suggests low condition number. This well condition behavior also exists among the other degrees of freedom and results in a reduced convergence time to find the minimum within the search space.

Iterative optimization algorithms are normally adopted to find this minimum. Although second-order Newton method is able to find the solution in fewer steps, it requires evaluation of complex quadratic norm defined as the Hessian matrix $\nabla^{2}\left\|\mathbf{w}_{\mathbf{n}}\right\|$, which is quite complex. Since the problem is well-conditioned, even the low complexity first-order Gradient decent method can find the solution quickly. If the iteration number is represented as superscript $k$, then the weights update in the $k^{\text {th }}$ iteration for Gradient descent method is given as

$$
w^{(k+1)}=w^{(k)}+t^{(k)} \Delta w^{k}
$$

where $t^{(k)}$ is the scalar step size obtained through backtracking method which reduces as one gets closer to the global minimum. $\Delta w^{k}$ is the step direction, which is chosen to maximize the negative gradient $\nabla\left\|\mathbf{w}_{n}^{(k)}\right\|$ at each step. The method descends in each step such that $\left\|\mathbf{w}_{n}^{(k+1)}\right\| \leq\left\|\mathbf{w}_{n}^{(k)}\right\|$, until the optimum minimum is reached, and the slope becomes positive. Since the objective function is trying to minimize the norm, the number of steps required for convergence is reduced if the starting point is chosen close to the origin. It should be noted that in this work, the algorithm was used to calculate the required compensated phases only (with uniform amplitudes). Computing the weights for phase correction using Eq. (7) to maximize the broadside gain of conformal antenna array in Figure 2 are used in Eq. (5) to plot the corrected radiation pattern.

\section{Analytical and simulation results}

To analytically compute the corrected (compensated) radiation pattern and validate with simulation results, the compensated array factor $\mathrm{AF}_{c}$ in Eq. (5) was used for different radii of curvatures of the cylindrical surface in Figure 2. 
The compensated (corrected) weights were computed using projection method in Section 2 and convex optimization in Section 3. The analytical results using Eq. (5) and CST simulation results using Figure 2 are discussed next.

\subsection{Cylindrical surface with radius of curvature $r=8 \mathrm{~cm}$}

The four-element microstrip patch antenna array on a cylindrical surface with radius $r=8 \mathrm{~cm}$ in Figure 2 was simulated in CST simulator. The inter-element spacing was $L=\lambda / 2$ at $f=2.45 \mathrm{GHz}$. The compensated phases for broadside pattern recovery were computed using Eq. (2) for projection method, and are tabulated in Table 1. For uncorrected pattern, the complex weights $w_{n}=1 \angle 0^{\circ}$ were applied on all antenna elements in CST simulator. Next, the weights (phase correction only) for broadside pattern recovery were calculated using the optimization algorithm in Section 3 and are also tabulated in Table 1. The analytical and CST simulation results for $r=8 \mathrm{~cm}$ are shown in Figure 4. Next, the difference in gain $\left(G_{r e f}\right)$ between flat array and compensated gain of conformal array were calculated and are also given in Table 1. It can be seen from Figure 4 and Table 1 that the broadside compensated gain $G_{c}$ of conformal antenna array is greater than the uncorrected gain and is less than the gain of flat array using both projection and convex optimization (phase correction only) methods. This is the fundamental limitation of both the compensation methods for broadside pattern recovery of conformal antenna arrays and should be kept in mind while designing conformal antenna array. It should be noted that convex optimization has more degrees of freedom than projection method in the sense that convex optimization gives complex weights (amplitude tapering plus phase correction), while projection method gives only phase correction (one degree of freedom). However, for broadside pattern recovery, convex optimization gives uniform amplitudes (equal to 1) and compensated phases and thus its performance is equal to projection method for broadside pattern recovery.

\begin{tabular}{|c|c|c|c|}
\hline$r(\mathrm{~cm})$ & Parameter & Projection method & Convex optimization \\
\hline \multirow[t]{3}{*}{8} & $\delta^{c}(\mathrm{deg})$ & {$[121.67,0,0,121.67]$} & {$[-89.49,146.95,146.95,-89.49]$} \\
\hline & $G_{c}(\mathrm{~dB})$ & 6.17 & 6.17 \\
\hline & $G_{r e f}(\mathrm{~dB})$ & 0.8 & 0.8 \\
\hline \multirow[t]{3}{*}{10} & $\delta^{c}(\mathrm{deg})$ & {$[101.84,0,0,101.84]$} & {$[-170.44,85.95,85.95,-170.44]$} \\
\hline & $G_{c}(\mathrm{~dB})$ & 4 & \\
\hline & $G_{r e f}(\mathrm{~dB})$ & 0.53 & \\
\hline \multirow[t]{3}{*}{12} & $\delta^{c}(\operatorname{deg})$ & {$[86.95,0,0,86.95]$} & {$[114.61,26.05,26.05,114.61]$} \\
\hline & $G_{c}(\mathrm{~dB})$ & 2.74 & \\
\hline & $G_{r e f}(\mathrm{~dB})$ & 0.4 & \\
\hline \multirow[t]{3}{*}{15} & $\delta^{c}(\mathrm{deg})$ & {$[70.95,0,0,70.95]$} & {$[9.66,-62.66,-62.66,9.66]$} \\
\hline & $G_{c}(\mathrm{~dB})$ & 1.765 & \\
\hline & $G_{r e f}(\mathrm{~dB})$ & 0.24 & \\
\hline \multirow[t]{3}{*}{30} & $\delta^{c}(\mathrm{deg})$ & {$[36.42,0,0,36.42]$} & {$[-102.2,-139.3,-139.3,-102.2]$} \\
\hline & $G_{c}(\mathrm{~dB})$ & 0.5 & \\
\hline & $G_{r e f}(\mathrm{~dB})$ & 0 & \\
\hline
\end{tabular}

Table 1.

Computed parameters of conformal cylindrical array for various radii of curvatures. 


\subsection{Cylindrical surface with radii of curvatures $r=10,12,15 \mathrm{~cm}$}

When radii of curvatures of cylindrical surface array increase (less deformation), both the projection and convex optimization (phase correction only) methods recover the broadside radiation pattern with decreasing gap between corrected and uncorrected gains as shown in Figures 5-7 and are also tabulated in Table1. The gain $G_{c}$ (between uncorrected and corrected) and $G_{r e f}$ (between corrected and linear) decreases with increase in $r$. However, it is obvious that in all cases, the
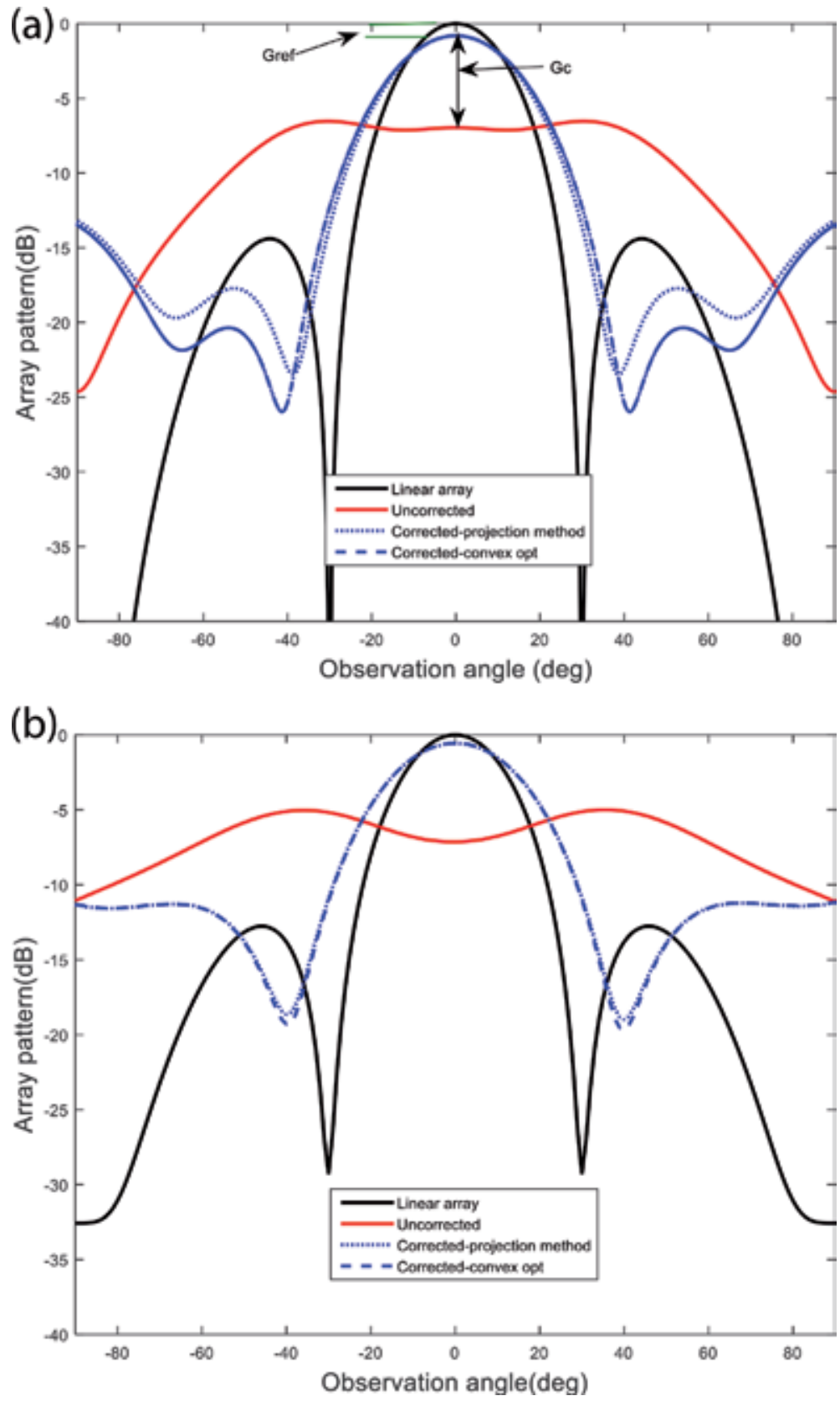

Figure 4.

(a) Analytical results for phase compensation of a conformal cylindrical antenna array with $r=8 \mathrm{~cm}$. (b) CST simulation results for phase compensation of a conformal cylindrical antenna array with $r=8 \mathrm{~cm}$. 

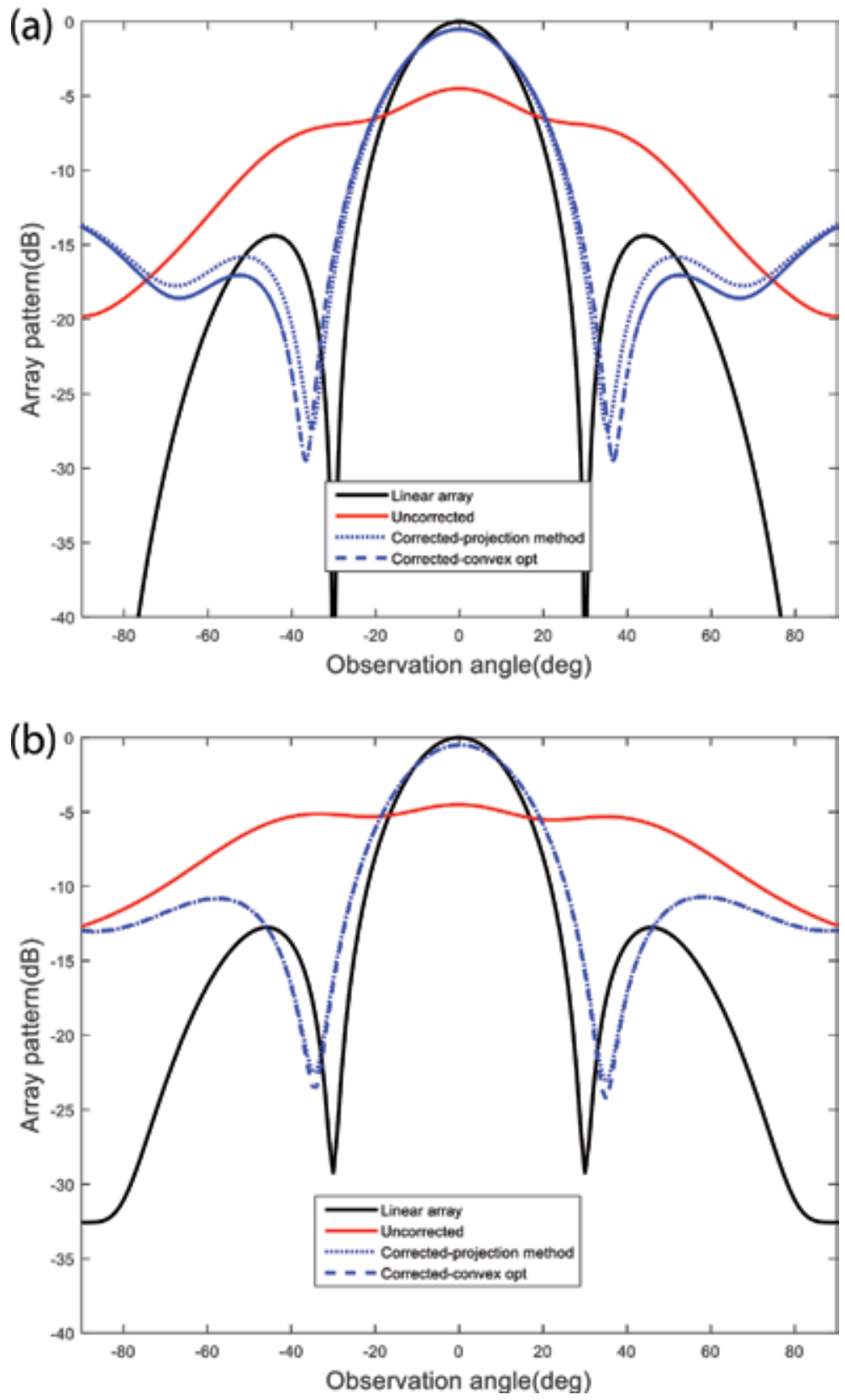

Figure 5.

(a) Analytical results for phase compensation of a conformal cylindrical antenna array with $r=10 \mathrm{~cm}$.

(b) CST simulation results for phase compensation of a conformal cylindrical antenna array with $r=10 \mathrm{~cm}$.

compensated gain is less than the ideal gain (linear array) for both projection and convex optimization methods. This is an important finding and must be kept in design stages of conformal antenna arrays.

\subsection{Cylindrical surface with radii of curvatures $r=30 \mathrm{~cm}$}

In the limiting case, when the radius of curvature of conformal cylindrical array increases up to $30 \mathrm{~cm}$ and above (approaching flat array), the compensated gains 

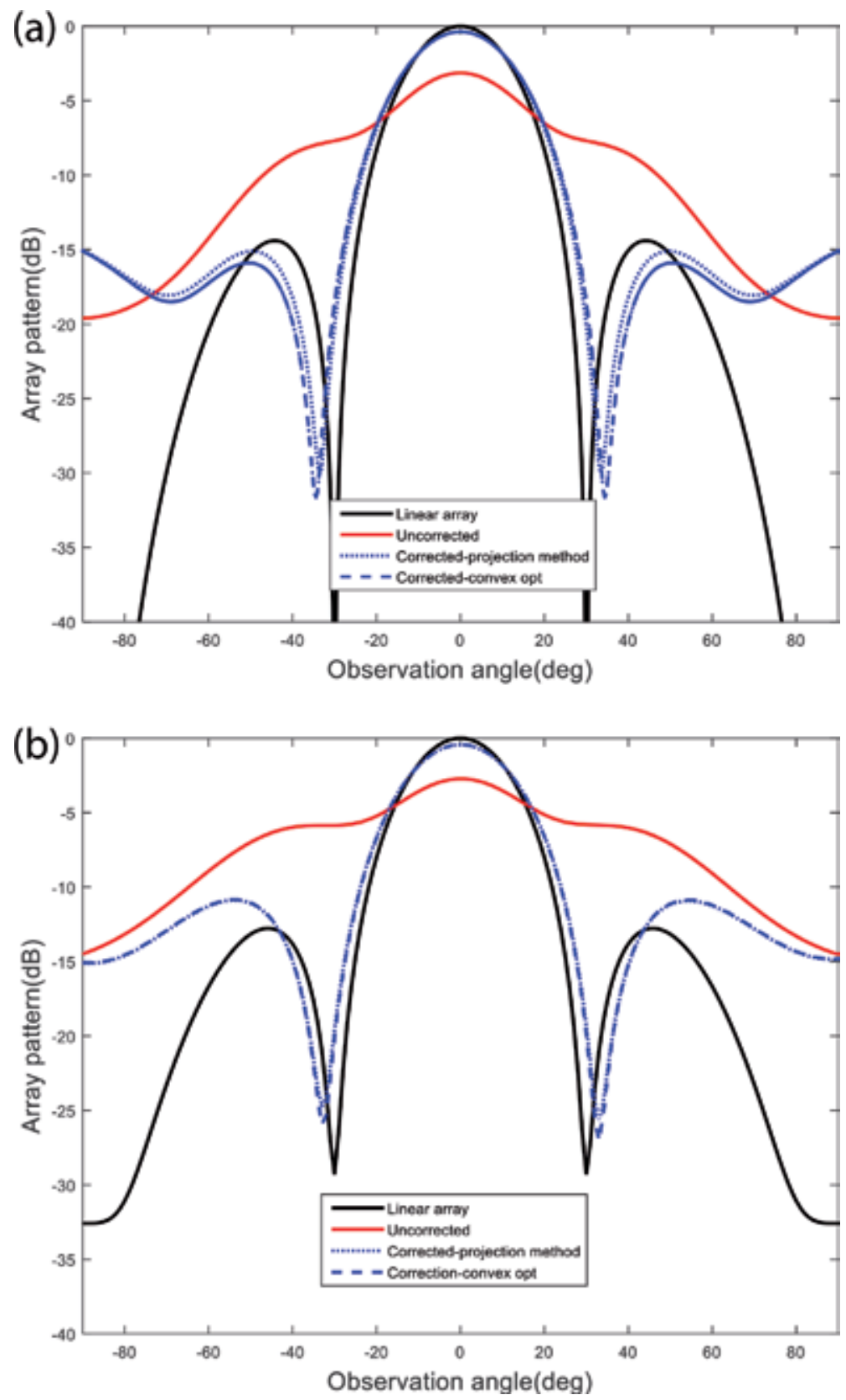

Figure 6.

(a) Analytical results for phase compensation of a conformal cylindrical antenna array with $r=12 \mathrm{~cm}$.

(b) CST simulation results for phase compensation of a conformal cylindrical antenna array with $r=12 \mathrm{~cm}$.

achieved from both projection and convex optimization methods nearly reaches the linear flat array gain, which is demonstrated in Figure 8 and is shown in Table 1.

\section{Conclusion}

In this chapter, phase compensation techniques based on projection method and convex optimization (phase correction only) have been discussed for recovery 

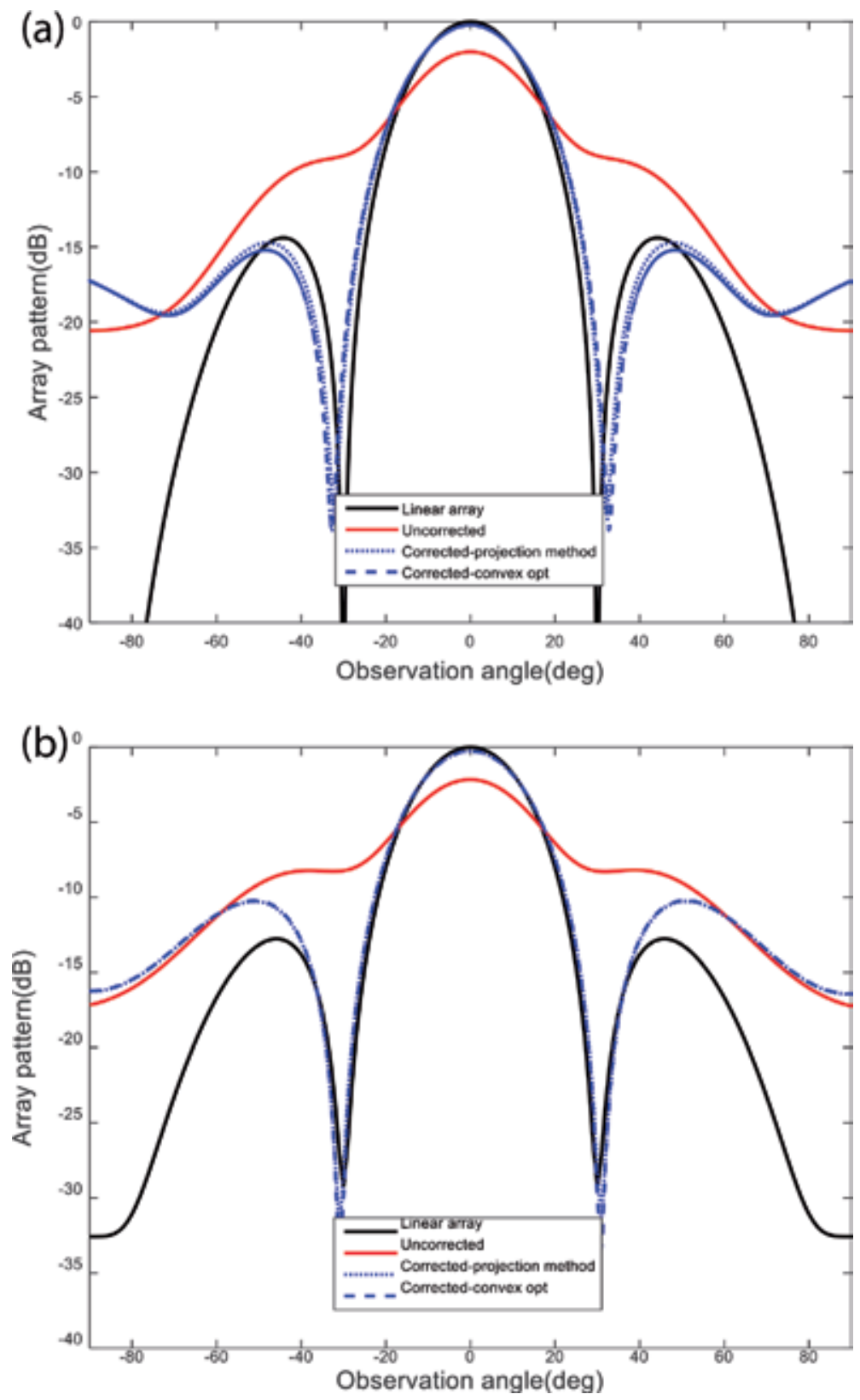

Figure 7.

(a) Analytical results for phase compensation of a conformal cylindrical antenna array with $r=15 \mathrm{~cm}$.

(b) CST simulation results for phase compensation of a conformal cylindrical antenna array with $r=15 \mathrm{~cm}$.

of broadside radiation pattern on a conformal cylindrical-shaped antenna array. The compensated gains of both the methods have been compared with linear flat antenna array. It is shown that the maximum broadside gain recovered with both the methods is less than the linear antenna array for severe deformation cases and approaches the gain of linear antenna array for less conformal deformation surfaces. The analytical expressions and convex optimization algorithm used can be used by a designer to predict the maximum possible compensated gain of conformal antenna array. 

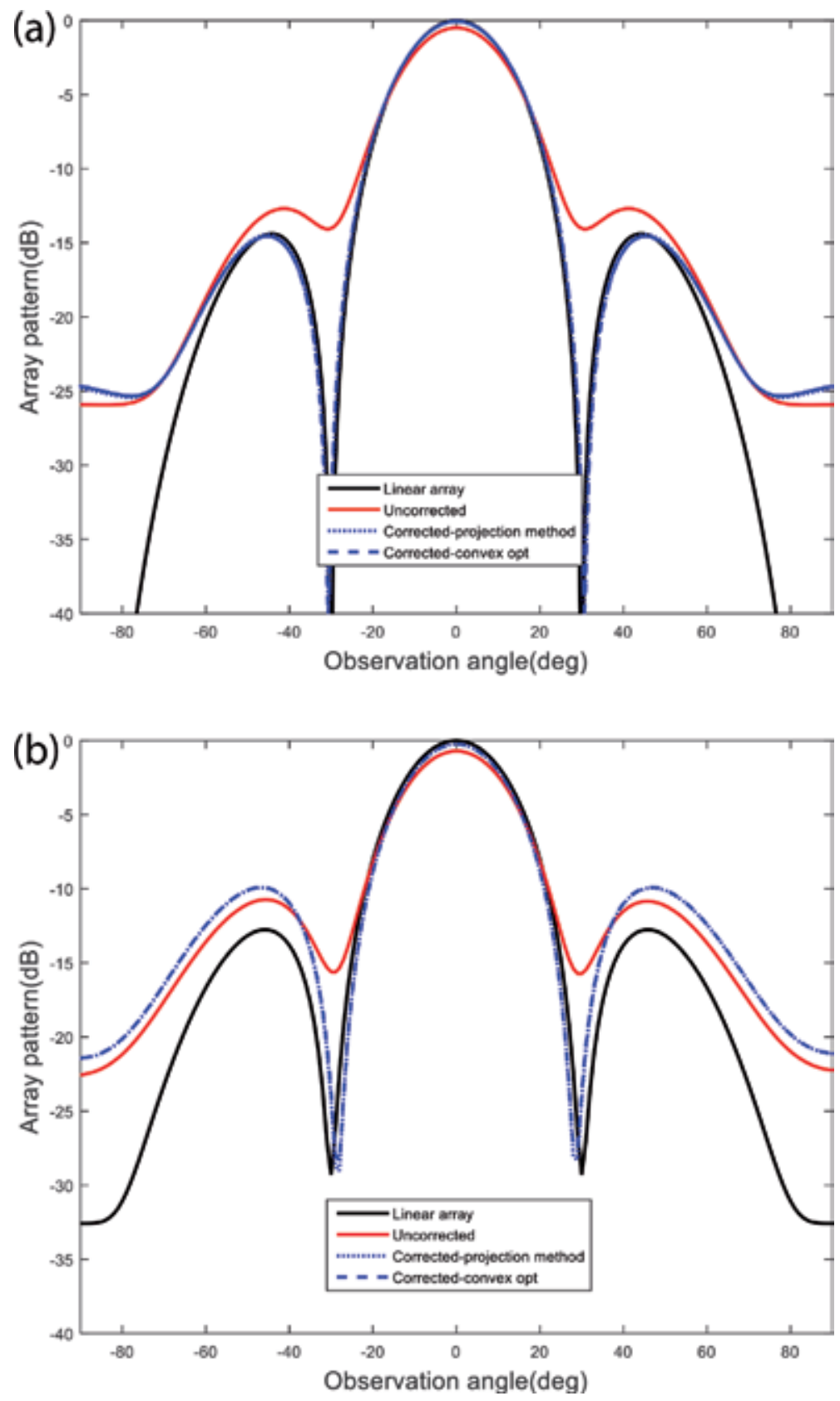

Figure 8.

(a) Analytical results for phase compensation of a conformal cylindrical antenna array with $r=30 \mathrm{~cm}$.

(b) CST simulation results for phase compensation of a conformal cylindrical antenna array with $r=30 \mathrm{~cm}$.

\section{Future work}

The proposed techniques can be extended for broadside pattern correction of conformal antenna arrays on other deformed surfaces (spherical nose of plane, flexing wings of UAV, etc.). Another interesting research can be to extend these techniques for beamforming on conformal deformed surfaces (e.g., on base station/ tower of cellular companies) to improve the signal-to-noise ratio (SNR) and its 
capacity. In this work, convex optimization is used to compute the compensated phases (with uniform amplitudes constraint) for recovery of broadside radiation pattern only. In practice, the technique is robust and can be used to calculate complex weights (amplitude tapering as well as phase correction), which can be further explored for beamforming applications and side lobe level control of conformal antenna arrays.

\section{Acknowledgements}

This work is supported by Ignite (NTF), Ministry of IT \& Telecom, Government of Pakistan via project no. ICTRDF/TR\&D/2015/04.

\section{Author details}

Irfan Ullah $^{1 *}$, Shahid Khattak ${ }^{1}$ and Benjamin D. Braaten ${ }^{2}$

1 Electrical and Computer Engineering Department, COMSATS University Islamabad, Abbottabad, Pakistan

2 Electrical and Computer Engineering Department, North Dakota State University, Fargo, USA

*Address all correspondence to: eengr@cuiatd.edu.pk

\section{IntechOpen}

(C) 2020 The Author(s). Licensee IntechOpen. This chapter is distributed under the terms of the Creative Commons Attribution License (http://creativecommons.org/licenses/ by/3.0), which permits unrestricted use, distribution, and reproduction in any medium, provided the original work is properly cited. (cc) BY 


\section{References}

[1] Schippers H, Knott P, Deloues T, Lacomme P, Scherbarth MR. Vibrating antennas and compensation techniques Research in NATO/RTO/SET 087/RTG 50. In: IEEE Aerospace Conference; 3-10 March 2007; Big Sky, MT. pp. 1-13

[2] Wincza K, Gruszczynski S. Influence of curvature radius on radiation patterns in multibeam conformal antennas. In: Proceedings of the 36th European Microwave Conference; 10-15 September 2006; Manchester. pp. 1410-1413

[3] Loecker C, Knott P, Sekora R, Algermissen S. Antenna design for a conformal antenna array demonstrator. In: 6th European Conference on Antennas and Propagation (EuCAP); 26-30 March 2012; Prague. pp. 151-153

[4] Salonen P, Rahmat-Samii Y, Schaffrath M, Kivikoski M. Effect of textile materials on wearable antenna performance: A case study of GPS antennas. In: IEEE International Symposium on Antennas and Propagation; 20-25 June 2004; Monterey. pp. 459-462

[5] Kennedy TF, Fink PW, Chu AW, Champagne NJ, Lin GY, Khayat MA. Body-worn E-textile antennas: The good, the low-mass, the conformal. IEEE Transactions on Antennas and Propagation. 2009;57:910-918. DOI: 10.1109/TAP.2009.2014602

[6] Psychoudakis D, Lee GY, Chen $\mathrm{C}-\mathrm{C}$, Volakis JL. Estimating diversity for body-worn antennas. In: 3rd European Conference on Antennas and Propagation (EuCAP); 23-27 March 2009; Berlin. pp. 704-708

[7] Semkin V et al. Conformal antenna array for millimeter-wave communications: Performance evaluation. International Journal of Microwave and Wireless Technologies.
2017;9:241-247. DOI: $10.1017 /$

S1759078715001282

[8] Kuang Y, Yao L, Yu SH, Tan S, Fan XJ, Qiu YP. Design and electromagnetic properties of a conformal ultra wideband antenna integrated in three-dimensional woven fabrics. Polymers. 2018;10:1-10. DOI: 10.3390/ polym10080861

[9] Schippers H, Verpoorte J, Jorna P, et al. Conformal phased array with beam forming for airborne satellite communication. In: International ITG Workshop on Smart Antennas (WSA 2008); 26-27 February 2008; Vienna. pp. $343-350$

[10] Salas Natera MA et al. New antenna array architectures for satellite communications. In: Karimi M, editor. Advances in Satellite Communications. 1st ed. London, UK: IntechOpen; 2011. pp. 167-194. DOI: $10.5772 / 838$

[11] Wu Y, Warnick F, Jin C. Design study of an L-band phased array feed for wide-field surveys and vibration compensation on FAST. IEEE Transactions on Antennas and Propagation. 2013;61:3026-3033. DOI: 10.1109/TAP.2013.2254438

[12] Braaten BD, Aziz MA, Roy S, Nariyal S, Irfanullah, Chamberlain NF, et al. A self-adapting flexible (SELFLEX) antenna array for changing conformal surface applications.

IEEE Transactions on Antennas and Propagation. 2013;61:655-665. DOI: 10.1109/TAP.2012.2226227

[13] Anagnostou D, Iskander M.

Adaptive flexible antenna array system for deformable wing surfaces. In: IEEE Aerospace Conference; 7-14 March 2015; Big Sky, MT. pp. 1-6

[14] Irfanullah, Khattak S, Braaten BD. Improvement of the broadside radiation 
pattern of a conformal antenna array using amplitude tapering. Applied Computational Electromagnetics Society Journal. 2017;32:511-516

[15] Braaten BD, Roy S, Irfanullah, Nariyal S, Anagnostou DE. An autonomous self-adapting conformal array for cylindrical surfaces with a changing radius. In: IEEE Antennas and Propagation Society International Symposium (APSURSI); 6-11 July 2014; Memphis. pp. 1784-1785

[16] Braaten BD, Roy S, Irfanullah, Nariyal S, Anagnostou DE. Phase compensated conformal antennas for changing spherical surfaces. IEEE Transactions on Antennas and Propagation. 2014;62:1880-1887. DOI: 10.1109/TAP.2014.2298881

[17] Josefsson L, Persson P. Conformal Array Antenna Theory and Design. 1st ed. Hoboken, New Jersey, United States: Wiley-IEEE Press; 2006. 472 p. DOI: 10.1002/047178012X

[18] Haupt RL. Antenna Arrays, A Computational Approach. 1st ed. Hoboken, New Jersey, United States: Wiley-IEEE Press; 2010. 534 p. DOI: 10.1002/9780470937464

[19] Seidel TJ, Rowe WST, Ghorbani K. Passive compensation of beam shift in a bending array. Progress in Electromagnetics Research C. 2012;29:41-53

[20] Ferreira DB, de Paula CB, Nascimento DC. Design techniques for conformal microstrip antennas and their arrays. In: Kishk A, editor. Advancement in Microstrip Antennas with Recent Applications. 1st ed. London, UK: IntechOpen; 2013. pp. 1-31. DOI: 10.5772/53019

[21] Khaleel HR, Al-Rizzo HM, Abbosh AI. Design, fabrication, and testing of flexible antennas. In: Kishk A, editor. Advancement in Microstrip
Antennas with Recent Applications. 1st ed. London, UK: IntechOpen; 2013. pp. 363-382. DOI: $10.5772 / 50841$

[22] Irfanullah, Nariyal S, Roy S, Masud MM, Ijaz B, Iftikhar A, et al. A note on the fundamental maximum gain limit of the projection method for conformal phased array antennas. In: Proceedings of the IEEE International Conference on Wireless Information Technology and Systems (ICWITS); 11-16 November 2012; Maui, HI. pp. $1-4$

[23] Rigobello F, Mansutti G, Khan MS, Capobianco AD. Pattern recovering of conformal antenna array for strongly deformed surfaces. In: 11th European Conference on Antennas and Propagation (EuCAP); 19-24 March 2017; Paris. pp. 869-871

[24] Mansutti G, Rigobello F, Asif S, Khan MS, Capobianco AD, Galtarossa A. Main lobe control of a beam tilting antenna array laid on a deformable surface. International Journal of Antennas and Propagation. 2018:1-6. DOI: 10.1155/2018/2521953

[25] Mansutti G, Khan MS, Capobianco AD, Iftikhar A, Asif S. Selfadapting conformal phased array antennas for complex changing surfaces. Microwave and Optical Technology Letters. 2017;59:393-399. DOI: 10.1002/ mop.30301

[26] Chiba I, Hariu K, Sato S, Mano S. A projection method providing low sidelobe pattern in conformal array antennas. In: International Symposium Digest: Antennas and Propagation; 26-30 June 1989; San Jose. pp. 130-134

[27] Liang Z, Ouyang J, Yang F. A hybrid GA-PSO optimization algorithm for conformal antenna array pattern synthesis. Journal of Electromagnetic Waves and Applications. 2018;32:1601-1615. DOI: 10.1080/09205071.2018.1462257 
[28] Xu Z, Li H, Liu Q-Z. Pattern synthesis of conformal antenna array by the hybrid genetic algorithm. Progress in Electromagnetics Research (PIER). 2008;79:75-90. DOI: 10.2528/ PIER07091901

[29] Mandrić V, Rupčić S, Žagar D.

Optimization of the spherical antenna arrays. In: Proceedings of 54th International Symposium ELMAR2012; 12-14 September 2012; Zadar. pp. 287-292

[30] Haupt RL. Adaptive antenna arrays using a genetic algorithm. In: IEEE Mountain Workshop on Adaptive and Learning Systems; 24-26 July 2006;

Logan, UT

[31] Hansen RC. Phased Array Antennas. 1st ed. Logan, UT, USA: John Wiley \& Sons, Inc.; 2009. 551. p. DOI: $10.1002 / 9780470529188$

[32] Boyd S, Vandenberghe L. Convex Optimization. 1st ed. New York: Cambridge University Press; 2004. 716 p. DOI: 10.1017/CBO9780511804441 

Section 2

\section{Elements and Feed Network}





\title{
Design of Reconfigurable Multiple-Beam Array Feed Network Based on Millimeter- Wave Photonics Beamformers
}

\author{
Mikhail E. Belkin, Dmitriy A. Fofanov, \\ Tatiana N. Bakhvalova and Alexander S. Sigov
}

\begin{abstract}
In this chapter, elaborating the direction of designing photonics-based beamforming networks (BFN) for millimeter-wave (mmWave) antenna arrays, we review the worldwide progress referred to designing multiple-beam photonics BFN and highlight our last simulation results on design and optimization of millimeterphotonics-based matrix beamformers. In particular, we review the specialties of mmWave photonics technique in 5G mobile networks of Radio-over-Fiber (RoF) technology based on fiber-wireless architecture. In addition, the theoretical background of array antenna multiple-beam steering using ideal models of matrix-based phase shifters and time delay lines is presented including a general analysis of radiation pattern sensitivity to compare updated photonics beamforming networks produced on phase shifter or true-time delay approach. The principles and ways to optimized photonics BFN design are discussed based on the study of photonics BFN scheme including integrated $8 \times 8$ optical Butler matrix (OBM). All schemes are modeled using VPIphotonics Design Suite and MATLAB software tools. In the result of simulation experiments, the outcome is obtained that both the integrated optical Butler matrix itself and the BFN based on it possess an acceptable quality of beams formation in a particular $5 \mathrm{G}$ pico-cell.
\end{abstract}

Keywords: 5G mobile communication network, small cell, wideband millimeterwave antenna array, photonics-based beamforming network, computer-aided design

\section{Introduction}

Generally, antenna unit is a requisite of any on-air radio frequency system forming its service area and bandwidth capability. At present, implementing an active phased array antenna (PAA) [1] results in remarkably increased footprint and operation flexibility thanks to electronic beam steering function, which is realized by a beamforming network (BFN). Today, the global telecommunications industry is experiencing a stage of violent development associated with the becoming of the fifth-generation mobile communication networks (5G NR) [2-6], and it is planned that one of the milestones for 5G NR compared to available 4G LTE networks should be millimeter-wave (mmWave) communication with mobile radio 
terminals $[7,8]$. This approach should lead to a newer network design technology using Radio-over-Fiber (RoF) building concept as well as PAA-assisted remote stations (RS) and user terminals (UT) [8,9]. On this way, integrated and millimeter-wave (mmWave) photonics are extremely attractive technologies for realizing a PAA's interactive optical BFN due to its superior instantaneous operating bandwidth, immunity to electromagnetic interference, lightweight, and reconfigurability [3].

Following it, recently we designed photonics-based BFNs for ultrawide bandwidth mmWave (57-76 GHz) antenna arrays [10]. Elaborating the direction, in this chapter, we review the worldwide progress referred to designing multiple-beam photonic BFN and highlight our last simulation results on design and optimization of millimeter-photonics-based matrix beamformers. Thus, in the rest of the sections, the following topics are under consideration. In particular, Section 2 reviews the specialties of mmWave photonics technique in $5 \mathrm{G}$ mobile networks of RoF technology based on fiber-wireless (FiWi) architecture. In addition, Section 3 presents theoretical background of array antenna multiple-beam steering using ideal models of matrix-based phase shifters and time delay lines. Section 4 includes a general analysis of radiation pattern sensitivity to compare updated photonics beamforming networks produced on phase shifter or true-time delay (TTD) approach. The principles and ways to optimized photonics BFN design are discussed in Section 5 based on the photonics BFN scheme including integrated $8 \times 8$ optical Butler matrix $(\mathrm{OBM})$. All schemes are modeled using VPIphotonics Design Suite and MATLAB software tools. Finally, Section 6 concludes the chapter.

\section{Millimeter-wave photonics technique in 5G fiber-wireless networks}

Based on 4G LTE progress [3], 5G NR is in principle a novel stage of unprecedented technological innovation with ubiquitous speed connectivity. As a result, it is expected that 5G NR will radically transform a number of industries and will provide direct, super-speed connections between any users and any sensors and devices. By now, several reviews to analyze significant changes in the $5 \mathrm{G}$ NR approaches as compared to the existing 4G LTE networks have been published $[8,11]$ denoting a series of milestones. Developing this topic, Table 1 summarizes the results of the advanced analysis focusing on the investigations referred to a fronthaul network with mobile communication in mmWave-band.

The review of the current R\&Ds in 5G NR area convincingly demonstrates the consistent achievement of the designated in Table $\mathbf{1}$ milestones, which is reflected in a vast number of publications and emergence of commercial products. Among them, much attention is paid to radically expanding the available spectral bands up to mmWaves (see item 1 of Table 1) to promote the throughput of mobile communication system. Following this tendency, currently, the local telecommunications commissions of various countries are proposing and harmonizing the plans of frequency allocation in mmWave-band, which will be reviewed this year at the World Radio Conference (WRC-2019). Currently, for the 5G NR networks, it is planned to allocate two frequency bands (see Figure 1), coexisting with available 4G LTE systems in the 1-6 GHz band (the so-called "low range" (LR)) and new one in the mmWaves within the range of $24.5-86 \mathrm{GHz}$ according to [12] (the so-called "high range" (HR)).

Based on various investigations, let us review the key advantages and disadvantages of the mobile communication system operation in the millimeter range. The following are the advantages of the $5 \mathrm{G}$ mmWave mobile communication: 
- It provides larger bandwidth, and hence, more number of UT can be accommodated.

- Its coverage is not limited to the line of sight (LoS) as first-order scatter paths are viable.

- Channel sounding feature is employed to take care of different types of losses at mmWave frequencies so that $5 \mathrm{G}$ network operates satisfactorily thanks to the measurement or estimation of channel characteristics, which helps in successful design, development, and deployment of 5G network with necessary quality requirements.

- Antenna size is physically small, and hence, a large number of antennas are packed in small volume. This leads to the use of massive multiple input, multiple output (MIMO), or beam-steerable PAA in RS to enhance the capacity (see item 2 of Table 1).

\begin{tabular}{|c|c|c|}
\hline No. & Designation & Short description \\
\hline 1 & $\begin{array}{l}\text { Radically expanding the } \\
\text { available spectral bands }\end{array}$ & $\begin{array}{l}\text { Some superwide bandwidth cases in } 5 \mathrm{G} \text { access networks will } \\
\text { require contiguous carrier bandwidths. To support them, } \\
\text { additional carrier frequencies (below } 6 \mathrm{GHz} \text { ), as well as } \\
\text { mmWave RF carriers will be required }\end{array}$ \\
\hline 2 & $\begin{array}{l}\text { Using active antenna systems in } \\
\text { mmWave communication }\end{array}$ & $\begin{array}{l}\text { Following the tendencies of expanding the available spectral } \\
\text { bands and increasing user densification, mmWave } 5 \mathrm{G} \text { wireless } \\
\text { network infrastructure can be erected with a lot of small cell } \\
\text { sites controlled by the corresponding RSs. In order to avoid } \\
\text { inter-interference inside these cells, one of the promising } \\
\text { approaches is to equip the RS with beam-steerable PAA using } \\
\text { hundreds of antenna elements to form multiple directional } \\
\text { beams in omnidirectional space }\end{array}$ \\
\hline 3 & $\begin{array}{l}\text { Establishing optimized access } \\
\text { network architecture }\end{array}$ & $\begin{array}{l}\text { Following the milestone of item } 1 \text {, it is necessary to optimize } \\
\text { the access network architecture so that at the same time it will } \\
\text { provide high-quality communication with fixed and mobile } \\
\text { users subject to low charges for the building and maintenance } \\
\text { of networks. A promising candidate for solving the problem is } \\
\text { a RoF's FiWi architecture, already tested in } 4 \text { G LTE systems }\end{array}$ \\
\hline
\end{tabular}

Table 1.

The milestones in the way to transform $4 G L T E$ to $5 G N R$.

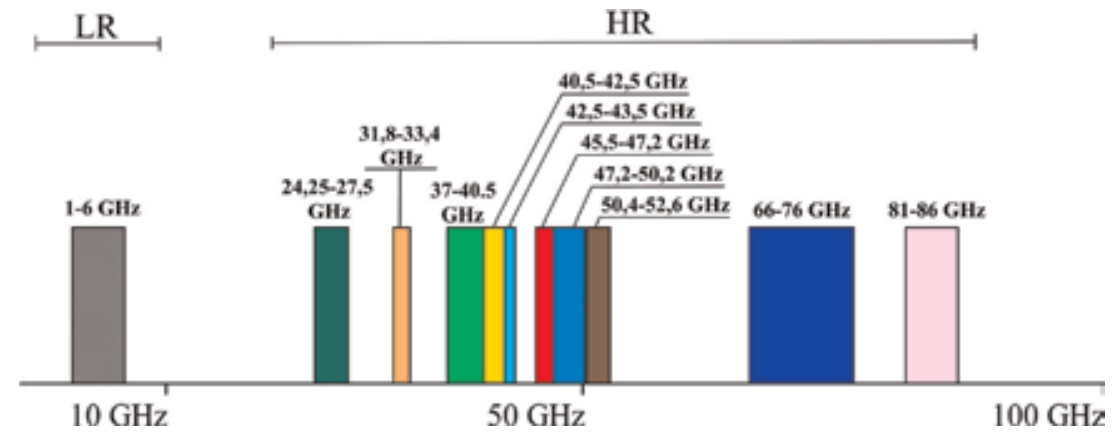

Figure 1.

Planned 5 G NR spectrum allocations [12]. 
- Dynamic beamforming is employed, and hence, it mitigates higher path loss at mmWave frequencies.

- 5G mmWave networks support multi-gigabit backhaul up to $400 \mathrm{~m}$ and cellular access up to 200-300 m [13].

Due to these benefits, $5 \mathrm{G}$ mmWave is suitable for mobile communication over sub-6 GHz wireless technologies. The main disadvantages of $5 \mathrm{G} \mathrm{mmWave} \mathrm{commu-}$ nication are the next:

- Millimeter-wave goes through different severe losses such as penetration, rain attenuation, and even foliage. This limits distance coverage requirement in 5Gbased cellular mobile deployment. Moreover, path loss is proportional to the frequency squared. It supports about $200-300 \mathrm{~m}$ in outdoors based on channel conditions and RS antenna height above the ground.

- It supports only LoS that limits the cell coverage.

- Power consumption is higher due to the greater number of RF modules and antennas. To avoid this drawback, hybrid architecture, which has fewer RF chains than the number of antennas, needs to be used at the RS receiver chain.

These disadvantages must be considered during 5G mmWave link budget calculation.

The drawbacks mentioned above led to the need for a radical change in the architecture of access networks compared to 4G LTE. In particular, instead of macro-cells, a multistage configuration was introduced, additionally containing micro-cells and pico-cells $[3,14]$. In this direction, a newer RoF-based access networks of FiWi architecture is considered as the most promising approach (see item 3 of Table 1) $([9,11])$. The reason is that the important drawback for the implementation of the wired links, for example, of Fiber-to-the-Home (FTTH) architecture is feasible for fixed UTs only. In contrast, current wireless access networks of 4G LTE that provide a flexible communication with a relatively simple infrastructure cannot meet growing in geometric progression demands to increase the capacity of mobile systems. The most promising technique to meet it, which is actively discussed in the referred publications, is to expand the operating frequency band and to apply multi-position digital modulation of a radio frequency (RF) carrier through fiber fronthaul to simplify pico-cell RS layout. Figure 2 illustrates a typical pico-cell in a large city. The mmWave wireless network is managed from a remote station including one unidirectional PAA for downlink and uplink channels.

\subsection{The outcome}

The source data for posterior calculations of multi-beam PAA in a pico-cell are:

- The base station is located on a separate mast of $3 \mathrm{~m}$ high in the geometric center of the service area (see Figure 2).

- The overall azimuth angle for the PAA under study is $360^{\circ}$.

- The elevation angle for the PAA under study must be such that the dead area around the mast does not exceed $1 \mathrm{~m}$. 




Figure 2.

Sketch of a typical wireless pico-cell for $5 G$ access network.

- The service radius of the pico-cell under investigation is $50 \mathrm{~m}$.

- The operating frequency band is $37.0-43.5 \mathrm{GHz}$ (see Figure 1).

\section{Theoretical background of multiple-beam array antenna beam steering}

As noted in chapter 2, mmWave array antennas capable of operating in ultrawide frequency range are considered as one of the key enabling technologies for designing RS of $5 \mathrm{G}$ NR network. There, a formation of a narrow steered beam by means of a PAA makes it possible to increase the directive gain to compensate for the excessive loss in the mmWave-band. Besides, the use of narrow beams would reduce the interference effects from other closely spaced mobile terminals and provides the possibility of spatial multiplexing to increase throughput while simultaneously exchanging information with several RSs.

Generally, electronic scanning in the PAA is provided by a beamforming network, which includes phase shifters or delay lines [1]. The BFN supports a continuous or discrete beam movement in space due to phase control or signal delay between the array elements. In our previous work devoted to the study of the PAA BFN [10], a single-beam PAA with electron scanning of the radiation pattern was considered. Nevertheless, for $5 \mathrm{G}$ pico-cells in conditions of simultaneous communication with a large number of terminal units, using a set of multiple-beam antenna (MBA) is considered to be a more practical way. PAAs based on MBA have greater functionality, but they are very complex, bulky, energy-consuming, and expensive devices. These factors limit their use to date mainly in special-purpose radars and unique satellite communication stations, for example, in satellite arrays of the iridium global mobile communication system [15]. There, PAA of the transponder has 106 channels and forms 16 fixed beams covering the contour-shaped 
the Earth's area. Each satellite has three such PAA, each of which forms its own sector. Thus, a set of 48 fixed satellite beams covers the Earth's area of about $4000 \mathrm{~km}$ in diameter.

As noted in [10], an appropriate beamforming scheme focusing the transmitted and/or received signal in a desired direction in order to overcome the unfavorable path loss is one of the key enablers for cellular communications in mmWave frequency bands. Depending on its layout, the beamforming weights required to form the directive beam could be applied in the digital or analog domain. Generally, digital beamforming provides a higher degree of freedom and offers better performance at the expense of increased complexity and cost because separate digital-toanalog converters, and analog-to-digital converters are required per each RF chain. Analog beamforming, on the other hand, is a simple and effective method of generating high beamforming gains from a large number of antennas but less flexible than digital counterpart.

For analog MBAs, BFN on the basis of multipole microwave circuits are usually applied. In particular, multipoles based on the Butler and Blass schemes are in common use since they are more compact than quasi-optical BFNs. In addition, they can be performed on printed circuit boards decreasing BFN's cost, size, weight, and power $(\mathrm{C}-\mathrm{SWaP})$ characteristics that are critical challenges in communication system design. For example, Butler matrix-based BFNs are exploited in the abovementioned iridium system. Currently, fixed-beam PAAs that use matrix BFNs based on a parallel circuitry (Butler matrix) and a serial circuitry (Blass matrix) [1] are being developed for photonics compatible mmWave small cell RSs of incoming 5G NR mobile communication networks.

Following this, below, a short theoretical study using ideal models is presented pursuing the goal to define the optimum RS's omnidirectional antenna construction, type, and configuration of multi-beam matrix for its BFN and the input data for the posterior design and optimization of the specific photonics-based BFN for the mmWave-band PAA exploiting widespread computer-aided design (CAD) tools.

First, following [1], the schematics and characteristics of Butler and Blass matrixes are discussed below.

\subsection{Butler matrix}

The traditional RF-band layout of Butler matrix consists of quadrature hybrids, fixed phase shifters, and transmission lines between them. A matrix can be used to feed a PAA; the number of elements of which is a multiple of degree 2. Figure 3

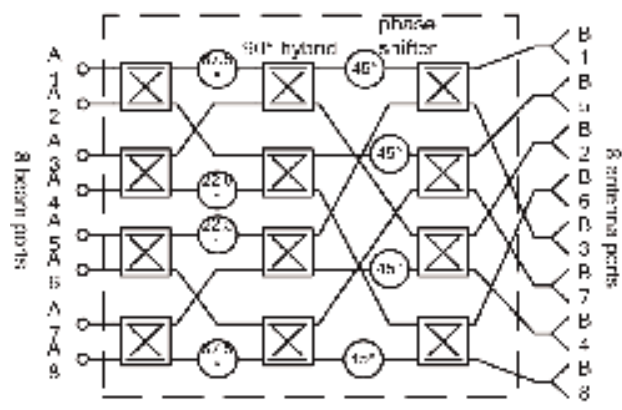

(a)

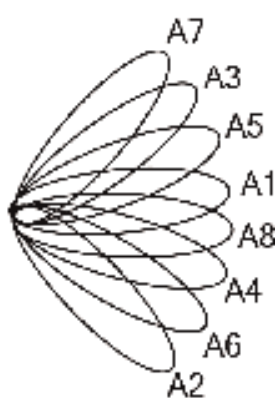

(b)

Figure 3 .

(a) Block diagram of $8 \times 8$ traditional Butler matrix and (b) corresponding BFN beam rosette. 


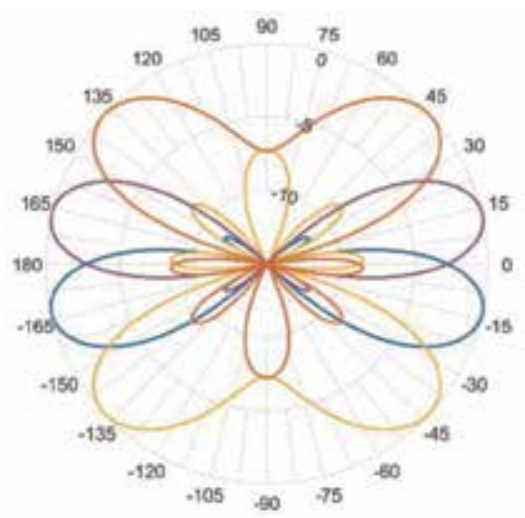

(a)

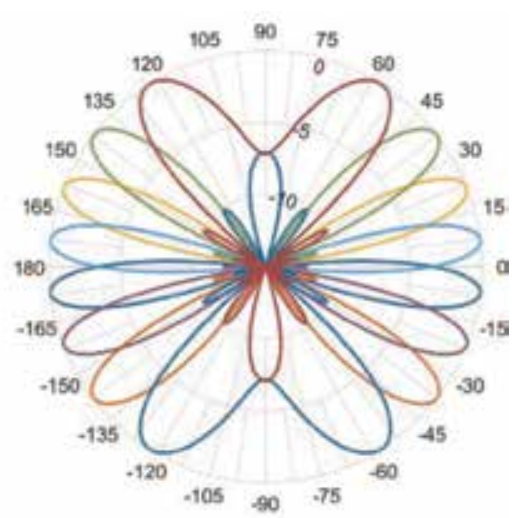

(b)

Figure 4.

Examples of the normalized radiation patterns for $4 \times 4(a)$ and $8 \times 8(b)$ Butler matrix.

demonstrates the block diagram (a) and BFN beam rosette (b) of the 8-element Butler matrix.

The number of inputs of the matrix is equal to the number of outputs. The amplitude-phase distribution at the outputs of the Butler matrix is described by the following formula:

$$
A_{n}=\frac{1}{\sqrt{N}} \sum_{m=1}^{N} e^{-j \frac{2 \pi}{N}(m-1)(n-1)}
$$

where $N$ is the number of channels; $m$ and $n$ are the number of the inputs and outputs, respectively. It should be noted that Eq. (1) is essentially a fast Fourier transform.

When connected to a linear equidistant PAA of $N$ omnidirectional element, the Butler matrix forms $N$ orthogonal beams, symmetrically located relative to the normal, with maxima in the azimuth directions $\varphi_{i}$ measured from the PAA broadside and determined by the formula:

$$
\cos \varphi_{i}=\left(i-\frac{N+1}{2}\right) \frac{\lambda}{L}, i=\overline{1 . . N}
$$

where $\lambda$ is the operating wavelength and $L$ is the PAA aperture. Moreover, the beams intersect each other at a level of $-4 \mathrm{~dB}$. As it follows from Eq. (2), the direction of the beams deviates when $\lambda$ varies, that is, a so-called squint effect is observed. Besides, the fan of orthogonal beams shrinks with decreasing $\lambda / L$ that is clearly seen in Figure 4 illustrating the normalized radiation patterns (NRP) for $4 \times 4$ (a) and $8 \times 8$ (b) Butler matrix calculated by MATLAB software.

Thus, due to the simplicity of the design and a relatively small number of elements, the Butler matrix is used in tasks that do not require the possibility of arbitrarily setting beam directions, for example, in covering the wide service sector of a wireless system.

\subsection{Blass matrix}

The Blass matrix consists of directional couplers connected to the inputs and outputs using transmission lines with different fixed delays. The matrix can be used to supply signals to the PAA with an arbitrary number of elements; the number of 
inputs can also be arbitrary and is determined by the required number of beams to be formed. The block diagram of the Blass matrix for three inputs and eight outputs, as well as the BFN beam rosette is shown in Figure 5.

The amplitude-phase distribution at the outputs of the Blass matrix with $N$ inputs is determined by the delays of the transmission lines $\tau_{m n}$ and the levels of the signals branched off each of the directional couplers $a_{m n}$ according to the formula:

$$
A_{n}=\sum_{m=1}^{N} a_{m n} e^{-j \omega \tau_{m n}},
$$

where $\mathrm{m}$ is the input number $\mathrm{n}$ is the output number.

Due to the fact that the RF signal from the input port sequentially passes through several directional couplers for feeding all the PAA elements, each coupler in the matrix must has the strictly defined value of the branch ratio, which greatly complicates the design. The configuration of the Blass matrix requires a larger number of directional couplers than Butler matrix, which increases its cost and often degrades the C-SWAP characteristics. However, due to the use of delay lines, the beams do not deviate from their position when the wavelength $\lambda$ varies as it happens using the Butler matrix (see Eq. (2)). For this reason, the Blass matrix is better feasible for ultrawide band systems with a fractional bandwidth of more than $20 \%$, as well as in systems requiring specific beam placement, for example, in satellite broadcasting equipment. Based on this outcome, in the course of further consideration of $5 \mathrm{G}$ mmWave MBA beam steering, only the BFN based on the Butler matrix will be studied.

\subsection{Antenna system for a mmWave pico-cell remote station of $5 \mathrm{G}$ mobile communication network}

From the outcome of Section 1, it follows that using an antenna's installation height of $3 \mathrm{~m}$ and a coverage radius of $50 \mathrm{~m}$, the elevation angle of $78^{\circ}$, provided by a half-wave dipole in the E-plane, is sufficient to provide a radius of not more than $0.5 \mathrm{~m}$ for the dead zone in the immediate vicinity of the mast (see Figure 6).

As can be seen in Figure 4, the extreme beams generated by the Butler matrix have a significantly greater width and less directivity than the others do. Their use

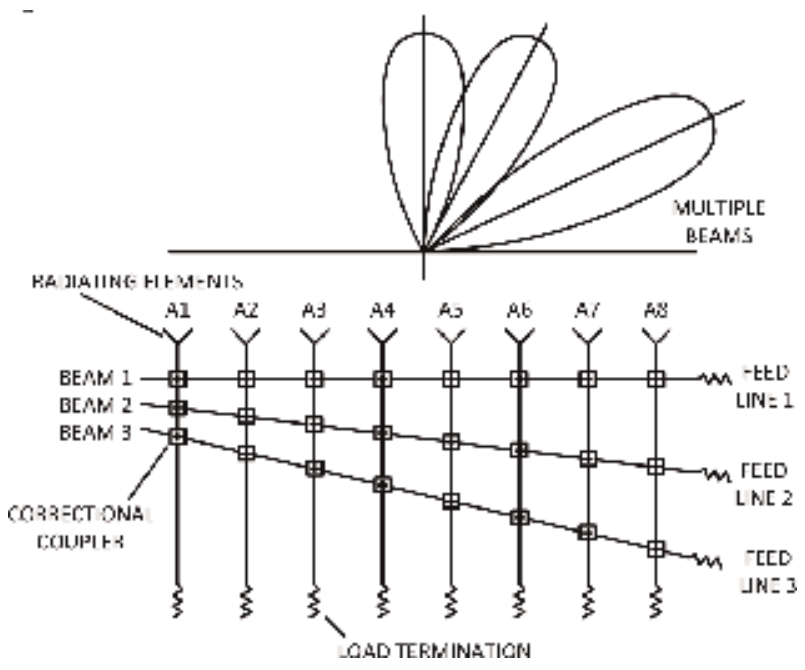

Figure 5 .

Block diagram of the $3 \times 8$ Blass matrix (bottom) and corresponding BFN beam rosette (top). 


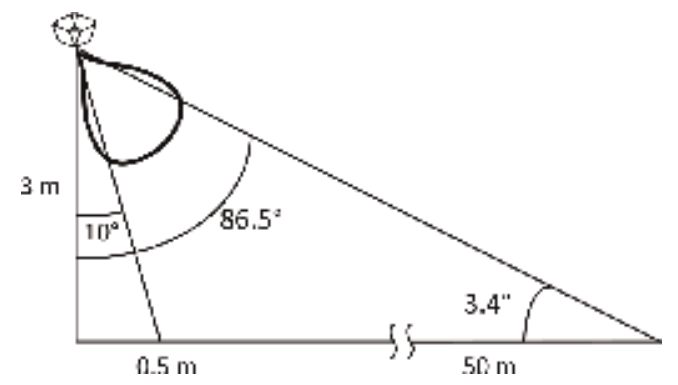

Figure 6.

Calculation of the radiation pattern in the elevation plane for one-dimensional PAA.

should be abandoned in order to avoid creating significant interference outside the service sector. Thus, the $4 \times 4$ matrix makes it possible to effectively exploit only two beams, which is not enough for spatial multiplexing of communication channels under the conditions illustrated in Figure 2; it is necessary to use an $8 \times 8$ matrix with six active channels. A fan using six beams allows covering a sector of the order of $50^{\circ}$ for the $-4 \mathrm{~dB}$ level (see Figure 4), which provides a full $360^{\circ}$ coverage with four PAAs mounted at $90^{\circ}$ relative to each other, as shown in Figure 7.

According to [1], the radiation pattern of a PAA $D(\theta, \varphi)$ is determined by the radiation pattern of a single antenna element $f(\theta, \varphi)$ and the array factor $F(\theta, \varphi)$ by the formula

$$
D(\theta, \varphi)=f(\theta, \varphi) * F(\theta, \varphi),
$$

where $\theta$ is an elevation angle and $\varphi$ is an azimuth.

For a half-wave dipole,

$$
\begin{gathered}
f(\theta, \varphi)=f(\theta) * f(\varphi) \\
f(\varphi)=\text { const }, \\
f(\theta)=\frac{1+\cos (\pi \cos \theta)}{\sin \theta}
\end{gathered}
$$

For a one-dimensional linear equidistant $8 \times 1$ PAA with a distance between elements $d=\lambda_{0} / 2$

$$
\begin{gathered}
F(\theta, \varphi)=F(\theta) * F(\varphi) \\
F(\theta)=\text { const, } \\
F(\varphi)=\sum_{n=1}^{8} A_{n} e^{\frac{2 \pi f f}{c} n \frac{\lambda}{2} \cos \varphi},
\end{gathered}
$$

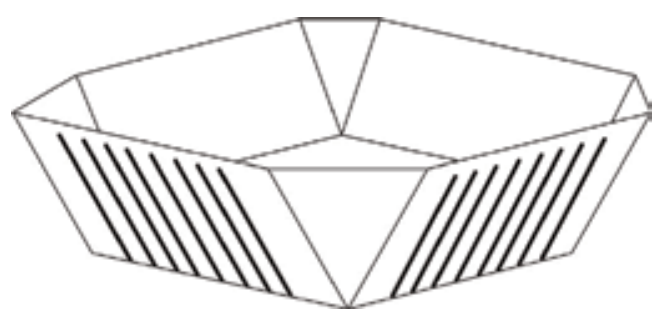

Figure 7.

Configuration of the antenna system for the mmWave pico-cell remote station under study. 
where $f$ is the signal frequency inside the operating frequency range of $37-43,5 \mathrm{GHz}, c$ is the light speed in vacuum, $\lambda_{0}$ is the wavelength corresponding to the center frequency of the operating frequency range, and $A_{n}$ is amplitudephase distribution generated by the Butler matrix and determined according to Eq. (2). Thus, the six-beam radiation pattern of single PAA is described by the formula:

$$
D(\theta, \varphi)=\frac{1+\cos (\pi \cos \theta)}{\sin \theta} * \sum_{n=1}^{8}\left[\frac{1}{\sqrt{8}} \sum_{m=1}^{6} e^{-j \frac{2 \pi}{N}(m-1)(n-1)}\right] e^{j \frac{2 \pi f}{c} \frac{\lambda_{0}}{2} \cos \varphi}
$$

Equation (7) is fundamental for further modeling.

Note that to ensure the required coverage in the elevation plane, the PAA panels have to be tilted to the ground at an angle near $45^{\circ}$. The unidirectional coverage provided in the azimuth plane by four sub-arrays of antenna system is illustrated in Figure 8.

To summarize, the following outcomes could be concluded:

- The Butler matrix is more suitable for the formation of a multipath radiation pattern in comparison with the Blass matrix because of its simpler design, fewer components, and better C-SWAP characteristics.

- The use of six central beams, generated by the eight-channel Butler matrix, provides a coverage sector of about $50^{\circ}$ and does not create a significant level of interference beyond its limits.

- The omnidirectional coverage of the service area is provided by using halfwave dipoles as elements of the one-dimensional PAA, providing coverage of $78^{\circ}$ in elevation angle and an antenna system of four linear PAA, providing overall coverage of $360^{\circ}$ in azimuth.

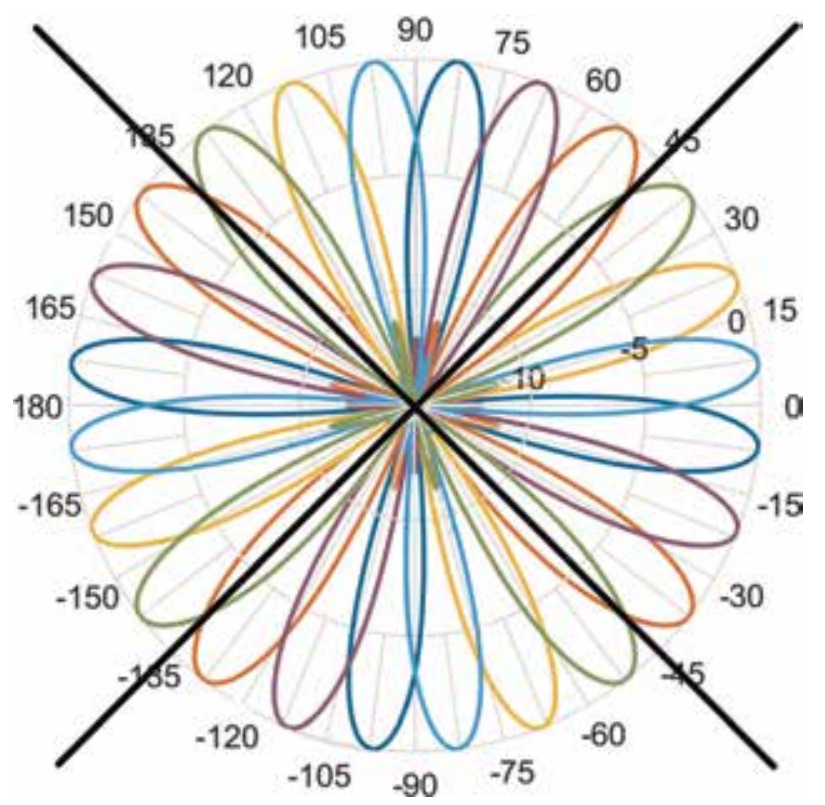

Figure 8.

Radiation pattern of RS antenna system in the azimuth plane. 


\section{A general analysis of radiation pattern sensitivity}

Due to the difficulty in providing time delays between PAA elements, phase shifters usually control the steering signal instead of using actual time delays, because their realization in RF band is much simpler, especially in the case of limited bandwidth. However, a phenomenon called "beam squint" leads to an error in the direction of the maximum of the PAA pattern and also to a certain increase in the level of the side lobes. Nevertheless, as known, a BFN based on phase shifters has become widespread in relatively narrowband RF-band PAAs with a fractional bandwidth, commonly not exceeding $10 \%$, depending on the criterion used [16]. Though, the development of a key trend for 5G NR networks associated with the implementing the mmWave in the wireless frontend has led to a change in the design principle of the access network's RS, whose antenna pattern was steered using photonics technique. At the same time, due to the more complexity for the implementation of fundamentally narrowbandwidth phase shifters in the optical range, the so-called true -time delay (TTD) concept based on wideband optical delay lines has been widely used [17-20].

Thus, when the fractional bandwidth of the BFN under design exceeds the $10 \%$ as noted above, it is required to determine the optimal approach by analyzing the sensitivity of the radiation pattern to the frequency change in the entire specific RF range. We previously performed this procedure for the mmWave PAA with singlebeam photonics BFN operating in the $57-76 \mathrm{GHz}$ RF band (fractional bandwidth of $28.6 \%$ ) [10]. As a result of the direct comparison, the TTD approach was unambiguously selected, since using phase shifters in the BFN produced more than $10 \%$ shift in the azimuth angle for the main lobe of the NRP, as well as increase in the side lobes level by almost $10 \mathrm{~dB}$. This chapter discusses a mmWave multiple-beam photonics BFN operating in the $37-43.5 \mathrm{GHz}$ band (fractional bandwidth of $16 \%$ ), for the implementation of which the Butler matrix (see Figure 3 ) is preselected (see section 3). In its scheme, to ensure the required phase shifts, optical delay lines of constant length are usually used [21]; therefore, prior to designing the specific BFN, the sensitivity analysis is also necessary.

In the process of simulation using MATLAB software, the sensitivity of the PAA's NRP is examined for the example of a linear equidistant array of eight ideal isotropic elements designed for operation at the center $(40.25 \mathrm{GHz})$ and two extreme ( 37.0 and $43.5 \mathrm{GHz}$ ) frequencies of the specified RF range. The BFN diagram was drawn based on the $8 \times 8$ Butler matrix according to Figure 3 with the replacement of phase shifters with ideal equivalent delay circuits, in which the constant delay $\Delta t$ was calculated at the center RF frequency $f_{c}$ using the following well-known formula:

$$
\Delta t=\Delta \varphi /\left(360 f_{c}\right)
$$

where $\Delta \varphi$ is the phase shift in degrees.

Table 2 lists the calculation results for phase shift (see Figure 3).

The results for MATLAB calculations of NRP using Eq. (7) at the center RF, lower RF, and upper RF in the azimuth angles range of $\pm 50^{\circ}$ from PAA broadside are shown in Figures 9-11, correspondingly.

\begin{tabular}{ccccc}
\hline Phase shift & $22.5^{\circ}$ & $45.0^{\circ}$ & $67.5^{\circ}$ & $90.0^{\circ}$ \\
\hline Time delay & $1.55 \mathrm{ps}$ & $3.1 \mathrm{ps}$ & $4.65 \mathrm{ps}$ & $6.2 \mathrm{ps}$ \\
\hline
\end{tabular}

Table 2.

Time delays of the equivalent delay circuits of $8 \times 8$ Butler matrix. 

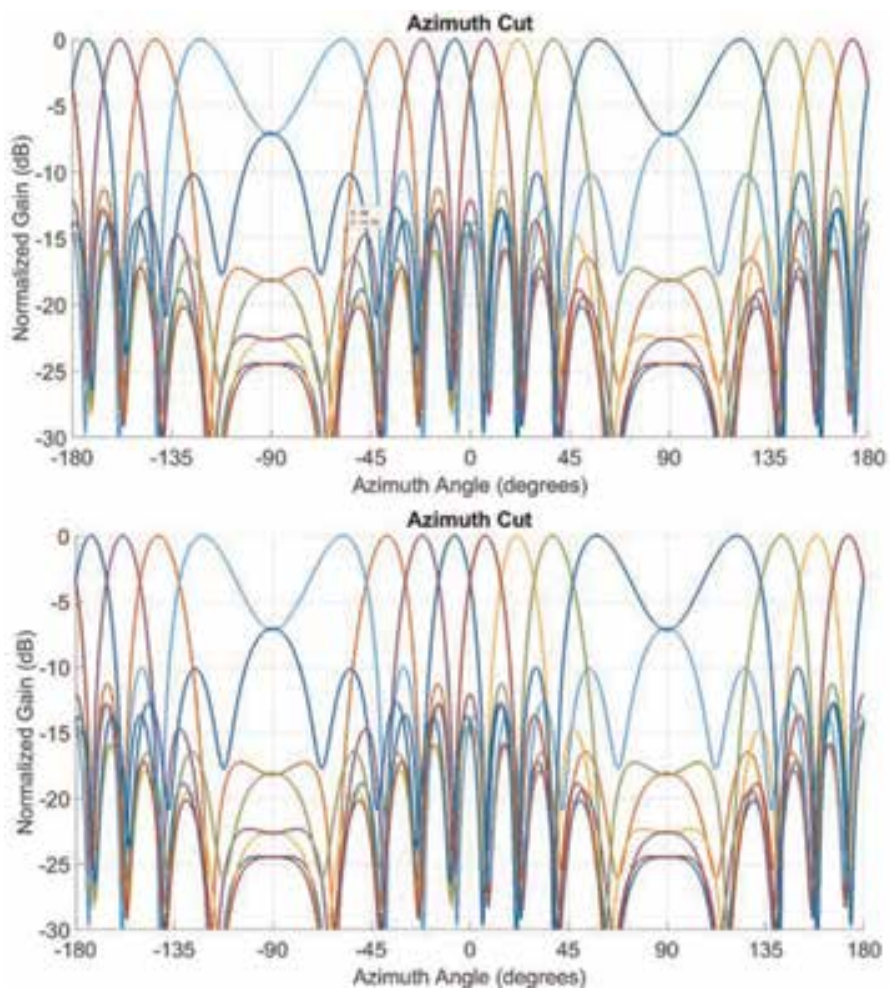

Figure 9.

$N R P$ at the center RF of $40.25 \mathrm{GHz}$ using phase matrix (top) or time delay matrix (bottom).
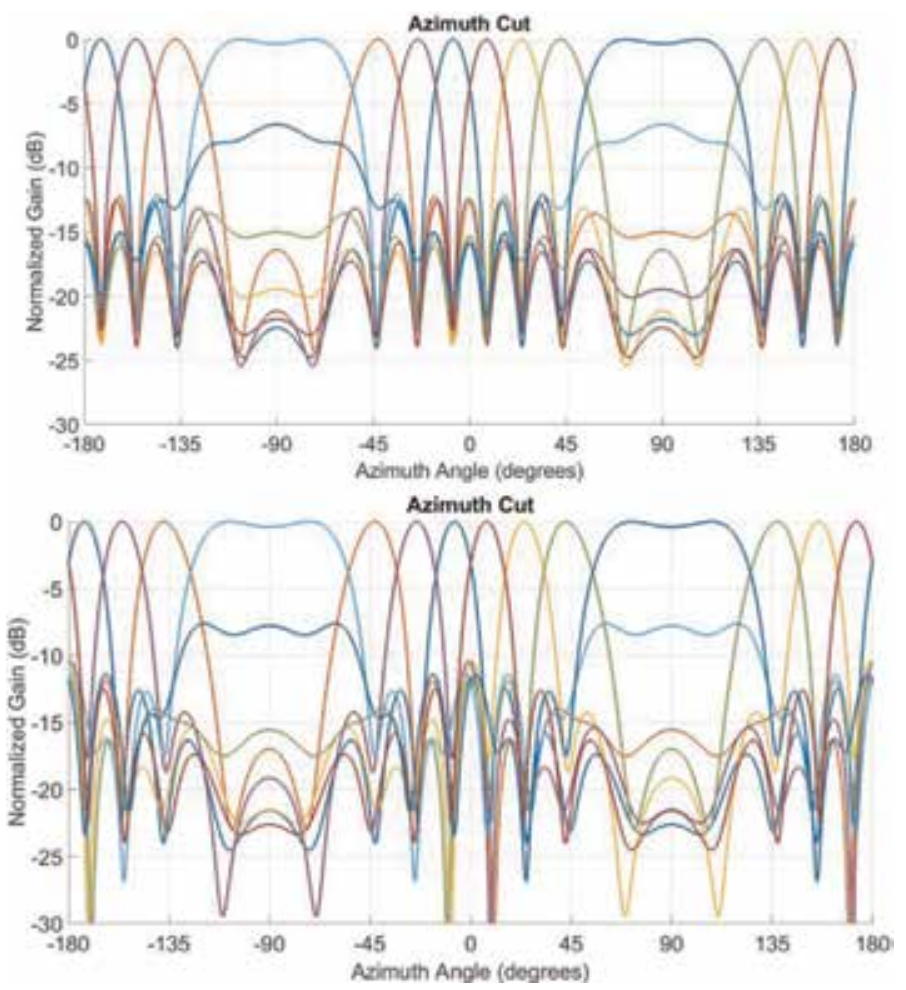

Figure 10.

NRP at the lower RF of $37.0 \mathrm{GHz}$ using phase matrix (top) or time delay matrix (bottom). 

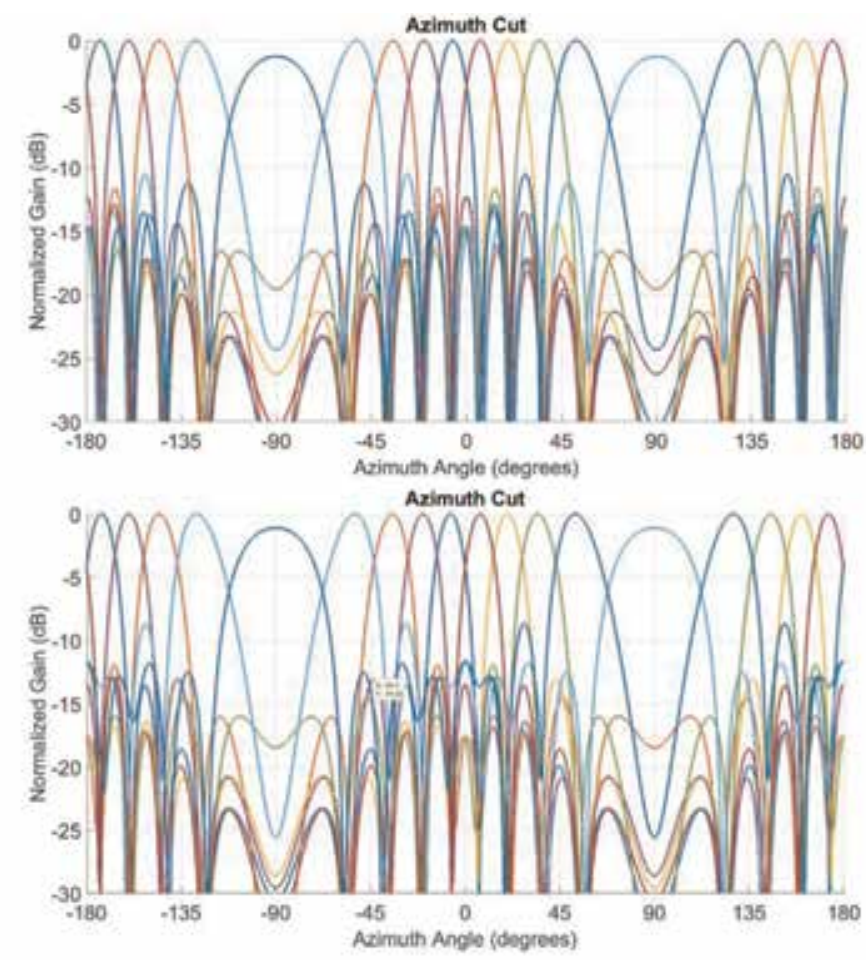

Figure 11.

NRP at the upper RF of $43.5 \mathrm{GHz}$ using phase matrix (top) or time delay matrix (bottom).

To summarize, the following outcomes could be concluded.

- According to [1], a Butler matrix provides a predetermined phase distribution at its outputs within the operating frequency band of its constituent components, such as quadrature hybrids and phase shifters. In it, when the RF deviates from the central one, the effect of beam squint is observed. The set of the beams narrows at the upper frequency and expands at the lower one, but the intersection point of the neighboring beams still remains at $-4 \mathrm{~dB}$ from the maximum.

- When used in the matrix, some delay elements with the values given in Table 2, the effect of the beam squint is not observed, and the positions of the maxima do not change with RF, but the radiation patterns lose orthogonality, and the beams have a greater overlap at the lower frequency and less at the highest one.

- Despite visible deviations in the shape of radiation patterns, the simulation results demonstrate the possibility of using delay elements in the Butler matrix to ensure uniform coverage of the sector $\pm 50^{\circ}$ in the $37-43.5 \mathrm{GHz}$ operating frequency range when the antenna elements are spaced through half the wavelength corresponding to the center frequency.

\section{Design principles and ways of integrated photonics-based millimeter-wave array beamformers}

In general, photonics-based BFNs for PAAs have many potential advantages over their electrical counterparts $[18,19,22]$, such as small size, low weight, no 
susceptibility to electromagnetic interference, and, especially, wide instantaneous bandwidth, and squint-free array steering while using TTD concept. This section first reviews the state of the art in mmWave photonic beamforming concepts and technologies and their potential application in multiple-beam antennas. Following it an updated schematic of multiple-beam mmWave array feed networks using photonics integrated circuit (PIC) of optical Butler matrix is proposed and modeling by well-known software tool VPIphotonics Design Suite [23].

To date several optical beamforming architectures have been proposed using different technological implementations [10] such as free-space optics, fiber optics, or integrated optics. Among them, integrated photonic beamformers (IPBF) are of particular interest from the point of view of compactness and moderate implementation costs [21, 24-28]. In addition, their attractiveness is expected to increase as the RF signal frequency increases up to mmWave. Today, a number of reviews and research papers are devoted to the study of building principles for 5G NR small cells in the mmWave band $[13,21,29,30]$. Table 3 highlights the main design principles and ways for mmWave IPBF.

The review of the referred sources allows us to conclude the following:

- The direction of mmWave IPBF is at the initial stage of its development. There are a small number of publications related to the research and development of IPBF in the field of telecommunications.

- There are two approaches to ensuring delays in an IPBF. The first is based on the transit time through the planar waveguide. The disadvantage of this method is the relatively large length of the waveguide, which leads to an attenuation of the signal and an increase in the dimensions of the beamformer. However, this method is often used due to the ease of implementation.

- The second approach involves the use of optical ring resonators. Its main disadvantage is narrowing the bandwidth with increasing group delay time, which leads to the necessity of cascading elements to obtain feasible delays. Nevertheless, with the help of ring resonators, it is possible to obtain an order of magnitude larger delay values.

\begin{tabular}{|c|c|c|c|c|c|c|}
\hline No. & $\begin{array}{c}\text { Time delay } \\
\text { unit }\end{array}$ & Scheme & Bandwidth & $\begin{array}{c}\text { Steering } \\
\text { method, } \\
\text { settling time }\end{array}$ & $\begin{array}{l}\text { Delay } \\
\text { range }\end{array}$ & Source \\
\hline 1 & $\begin{array}{l}\text { Integrated } \\
\text { waveguide }\end{array}$ & Binary with $2 \times 2$ switches & $\begin{array}{c}\text { Narrowband } \\
42.7 \mathrm{GHz}\end{array}$ & $\begin{array}{l}\text { Switchable, } \\
4 \text { bit, } 20 \mathrm{~ns}\end{array}$ & $15.7 \mathrm{ps}$ & {$[31]$} \\
\hline 2 & $\begin{array}{l}\text { Optical ring } \\
\text { resonator }\end{array}$ & $1 \times 4$ TTD binary tree & $\begin{array}{l}8.7 \mathrm{GHz} \text { at } \\
90 \mathrm{GHz}\end{array}$ & Thermal tuning & $172.4 \mathrm{ps}$ & [32] \\
\hline 3 & $\begin{array}{l}\text { Integrated } \\
\text { waveguide }\end{array}$ & $2 \times 2$ Butler matrix & $\begin{array}{l}\text { Approximately } \\
200 \mathrm{MHz}\end{array}$ & Fixed & $100 \mathrm{ps}$ & [21] \\
\hline 4 & $\begin{array}{l}\text { Optical ring } \\
\text { resonator }\end{array}$ & $16 \times 1$ TTD binary tree & $2.5 \mathrm{GHz}$ & Thermal tuning & $1200 \mathrm{~ns}$ & [33] \\
\hline 5 & $\begin{array}{l}\text { Integrated } \\
\text { waveguide }\end{array}$ & $8 \times 8$ Blass matrix & - & - & - & [33] \\
\hline 6 & $\begin{array}{l}\text { Integrated } \\
\text { PLC } \\
\text { waveguide }\end{array}$ & $\begin{array}{l}\text { Independent phase and } \\
\text { amplitude control, four } \\
\text { channels }\end{array}$ & $\begin{array}{l}\text { Narrowband, } \\
60.8 \mathrm{GHz}\end{array}$ & $\begin{array}{c}\text { Thermo-optic } \\
\text { effect }\end{array}$ & $\pm 45^{\circ}$ & {$[34]$} \\
\hline
\end{tabular}

Table 3.

Examples of $m m$ Wave IPBF. 


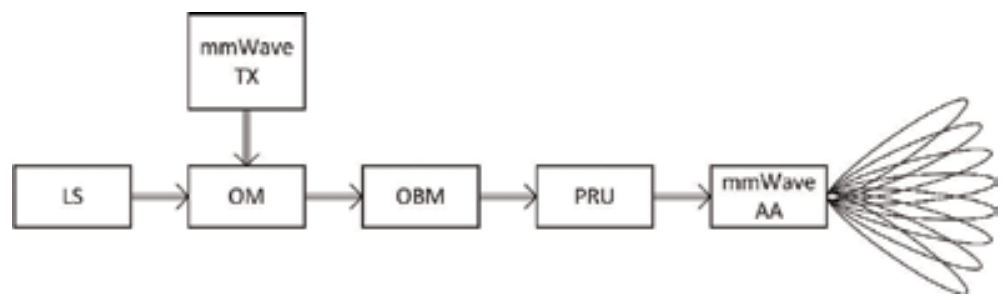

Figure 12.

Generalized block diagram of photonics-based mmWave multiple-beam array feed network.

- One of the most promising techniques for designing an RS's PAA is to use IPBFs based on a multiple-beam Butler matrix.

Analysis of the publications referenced in Table 3 allows us to draw a generalized block diagram of photonics-based mmWave multiple-beam array feed network for downlink channel of RS, which is shown in Figure 12.

As follows from the Figure, the principal units are the laser sources (LS), optical modulators (OMs) performing the operation of electro-optical conversion, and the intensity of the output signal for which is controlled by the mmWave transmitter (TX). The output optical signals of the OMs are fed to a spatial distribution unit based on $8 \times 8$ optical Butler matrix. A photoreceiver unit (PRU) is connected to its outputs performing the operations of reverse optical-to-electrical conversion and amplification of the mmWave electrical signal to a level sufficient for reliable radio communication within the pico-cell of Figure 2, which is performed using the array antenna (AA). Note that the uplink channel between UT and RS is designed in a similar way and can be simplified using the reciprocity property of the Butler matrix.

\subsection{Reference data for the simulation}

In this work, the subject of the study is a mmWave multiple-beam array feed network, and the device of the study is an integrated optical Butler matrix. A tool for the computer simulation is the well-known commercial software VPIphotonics Design Suite ${ }^{\mathrm{TM}}$. In the course of the research, first of all, the accuracy of creating a mmWave $8 \times 8$ integrated OBM is checked. Then, the transmission quality of a mmWave multiple-beam array feed network using this OBM through the downlink channel for one of four sectors of the pico-cell RS (see Figures $\mathbf{2}$ and 7) is analyzed by the simulation in VPI and MATLAB software. Table 4 lists the reference data for the integrated OBM under study and the setup for its characterization. In addition, Table 5 lists the reference data for the array feed network under analysis.

\subsection{CAD models and setups}

According to the outcomes in the previous section, when analyzing with the help of MATLAB software, before modeling the integrated OBM using VPIphotonics Design Suite environment, it is worth checking the phase shifts provided by the equivalent delay elements based on integrated waveguides. Figure 13 depicts the model that consists of one delay-less arm and the four arms with library models of TriPleX-based integrated waveguides $\left(n_{\mathrm{g}}=2.016\right)$ providing phase shift of $22.5^{\circ}$, $45^{\circ}, 67.5^{\circ}$, and $90^{\circ}$, correspondingly (see Figure 3a for the reference), and setup for the simulation experiments. In addition, there are two instrumental library models in the setup. The first one imitates optical transmitting module including library 


\begin{tabular}{lcc}
\hline \multicolumn{2}{l}{ Parameter } & Value \\
\hline Number of optical inputs & \multicolumn{1}{c}{8} \\
\hline Number of optical outputs & & 8 \\
\hline Band of RF carrier frequencies & & $37.5-41.0 \mathrm{GHz}$ \\
\hline Input RF power & & -11 to $-26 \mathrm{dBm}$ \\
\hline Material platform for IPBF & Responsivity & TriPleX $\left(\mathrm{Si}_{3} \mathrm{~N}_{4} / \mathrm{SiO}_{2}\right)[35]$ \\
\hline PIN photodiode & Dark current & $0.92 \mathrm{~A} / \mathrm{W}$ \\
\cline { 2 - 3 } & 3 dB bandwidth & $100 \mathrm{nA}$ \\
\cline { 2 - 3 } & Optical input power & $50 \mathrm{GHz}$ \\
\hline Laser source & Optical carrier & $<3 \mathrm{~mW}$ \\
\cline { 2 - 3 } & Average power & $193.1 \mathrm{THz}$ \\
\cline { 2 - 3 } & Linewidth & $50 \mathrm{~mW}$ \\
\hline Optical modulator & Principle & $10 \mathrm{kHz}$ \\
\cline { 2 - 3 } & Modulation type & Electro-absorption \\
\cline { 2 - 3 } & Spectral range & 0 \\
\cline { 2 - 3 } & Modulation index & $\mathrm{C}$ band \\
\cline { 2 - 3 } & Chirp factor & 0.5 \\
\hline
\end{tabular}

Table 4.

The reference data for the OBM under study and the setup for its characterization.

\begin{tabular}{lc}
\hline Parameter & Value \\
\hline Overall number of mobile UTs in the pico-cell & 72 \\
\hline Number of mobile UTs in one sector & 18 \\
\hline Number of PAA sectors & 4 (see Figure 7) \\
\hline Number of PAA beams in one sector & 6 \\
\hline Number of RF carrier frequencies & 6 \\
\hline Band of RF carrier frequencies & $38.0-40.5$ \\
\hline Spacing of RF carrier frequencies & $0.5 \mathrm{GHz}$ \\
\hline
\end{tabular}

Table 5 .

The reference data for the array feed network under analysis.

models of laser source and optical modulator EA controlled by RF generator tuning in the band of 37.5-41.0 GHz. The second one imitates optical receiving module including library models of PIN photodiode and RF network analyzer recording amplitude and phase RF signal distribution at the photodiode output. One can see their relevant parameters in Table 4.

Then, Figure 14 depicts the model and setup of $8 \times 8$ OBM that in according to Figure 3a contains the models of quadrature optical hybrids (QOH) and library models of the straight waveguide as a phase shifter.

Due to the lack of a suitable library model in this software tool, $\mathrm{QOH}$ is designed as a so-called "galactic" module G, containing, in accordance with a typical circuitry of an electrical analog, library models of two optical X-couplers and two optical straight waveguides with $90^{\circ}$ phase shift. Both elements are carried out based on 
Design of Reconfigurable Multiple-Beam Array Feed Network Based on Millimeter-Wave... DOI: http://dx.doi.org/10.5772/intechopen.89076

TriPleX technology. The internal scheme of the galactic module is presented in Figure 15. In addition, the setup of Figure 14 includes two instrumental library models, which are the same as in Figure 13.

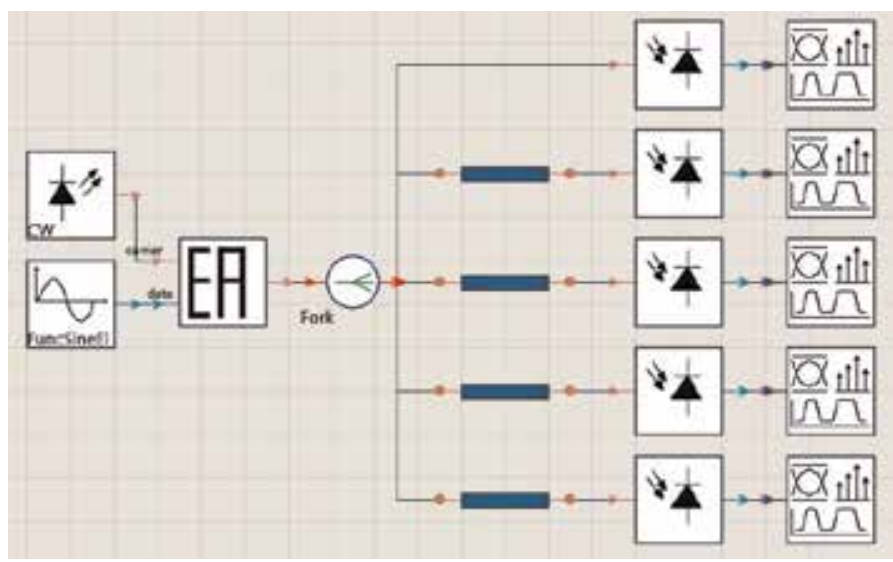

Figure 13.

Equivalent delay elements of integrated $O B M$.

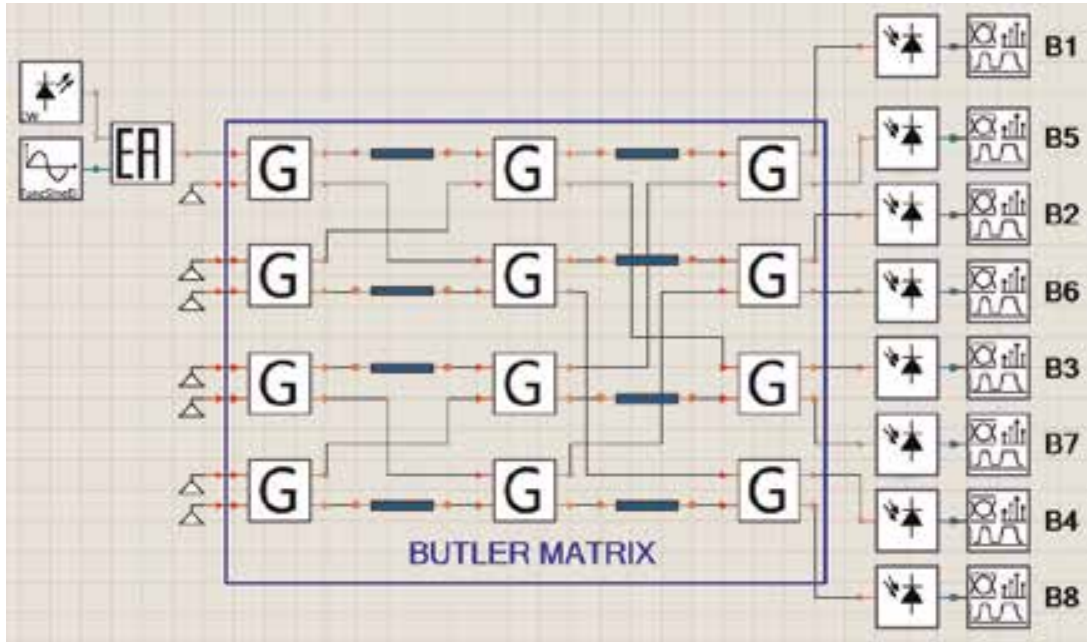

Figure 14 .

The model and setup for simulation of $8 \times 8$ PIC-based OBM.

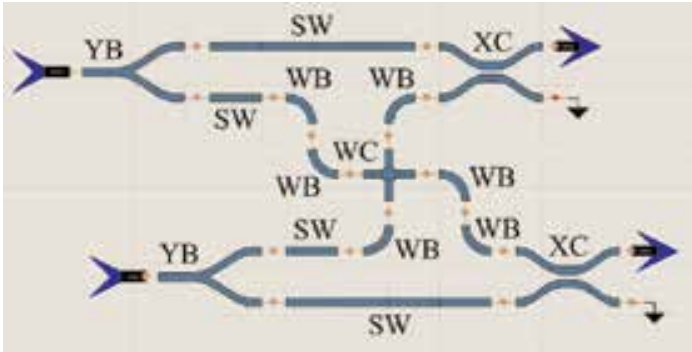

Figure 15.

The internal scheme for the galactic module $G$ of a quadrature optical hybrid. 


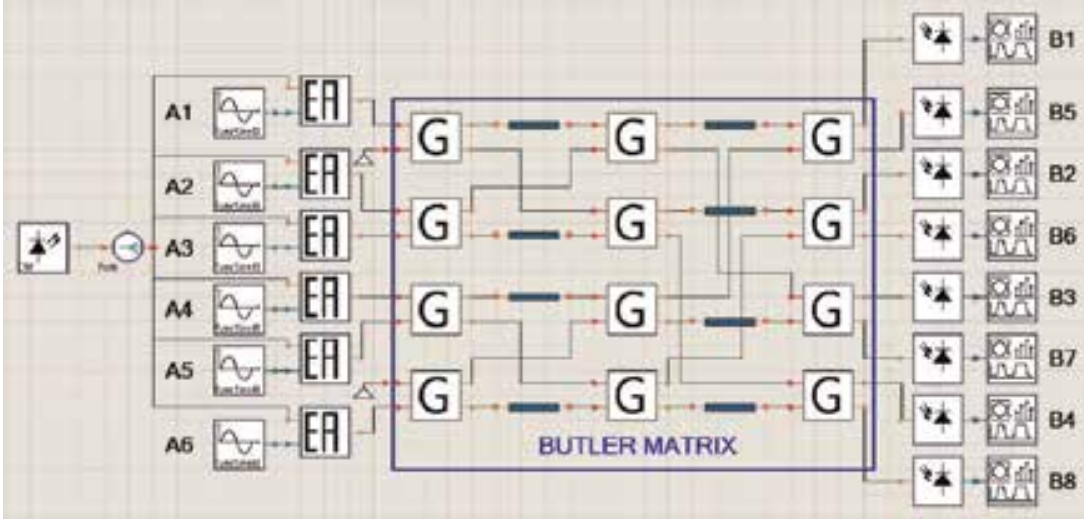

Figure 16.

The model and setup for simulation of mmWave multiple-beam array feed network.

The module of Figure 15 contains a set of PIC library models, such as two Y-branches (YB), four straight waveguides (SW) including two SW for $90^{\circ}$ phase shift, and two compensating SW with equivalent phase shift of $360^{\circ}$, six $90^{\circ}$ waveguide bends (WB), one waveguide crossing element (WC), and two $\mathrm{X}$-couplers (XC).

Finally, Figure 16 depicts the model and setup for the mmWave multiple-beam array feed network under study that contains the model of $8 \times 8$ OBM (see Figure 14) with six inputs because as shown in subsection 3.1, the extreme beams generated by the Butler matrix (A2 and A7 in Figure 3) have a significantly greater width and less directivity than the others do (see Figure 4). In addition, there are two instrumental library models in the setup. The first one imitates optical transmitting module including library models of laser source and six optical modulators controlled by six RF generators, the RF carriers of which are allocated in the band of 38.0-40.5 GHz. The second one imitates optical receiving module including library models of eight PIN photodiodes and eight RF network analyzers recording amplitude and phase RF signal distributions at the photodiode outputs. One can see their relevant parameters in Tables 4 and 5.

\subsection{Simulation results}

First, a simulation experiment for the delay elements of PIC-based OBM (see Figure 13) was carried out. Table 6 lists the results of phase error values for the center and two extreme frequencies of the RF generator.

Then, a simulation experiment for the PIC-based $8 \times 8$ OBM (see Figure 14) was carried out when the output of EA was alternately connected to each input of OBM, and at each point, the RF generator was sequentially tuned to the frequency of each

\begin{tabular}{lccccc}
\hline \multicolumn{2}{l}{ Reference phase shift } & $-22.5^{\circ}$ & $-45^{\circ}$ & $-67.5^{\circ}$ & $-90^{\circ}$ \\
\hline Equivalent lengths $(\mathrm{mm})$ & 0.215 & 0.437 & 0.662 & 0.883 \\
\hline Error value & At the center RF & $-0.1^{\circ}$ & $-0.2^{\circ}$ & $-0.4^{\circ}$ & $-0.5^{\circ}$ \\
\cline { 2 - 5 } At the lowest RF & $-1^{\circ}$ & $-2^{\circ}$ & $-3^{\circ}$ & $-4^{\circ}$ \\
\cline { 2 - 5 } At the upper RF & $1^{\circ}$ & $2^{\circ}$ & $3.1^{\circ}$ & $4.1^{\circ}$ \\
\hline
\end{tabular}

Table 6.

The simulation results of phase error values at the outputs of OBM under test. 
Design of Reconfigurable Multiple-Beam Array Feed Network Based on Millimeter-Wave... DOI: http://dx.doi.org/10.5772/intechopen.89076

downlink channel. Table 7 exemplifies the simulation results of phase error values for channel A6 (see Figure 16) at the corresponding outputs.

Finally, a simulation experiment for the mmWave multiple-beam array feed network of Figure 16 was carried out. Figure 17 exemplifies the calculation results of the back-baffled normalized radiation patterns generated at the central and two extreme frequencies of the input RF band based on the data for the amplitude and

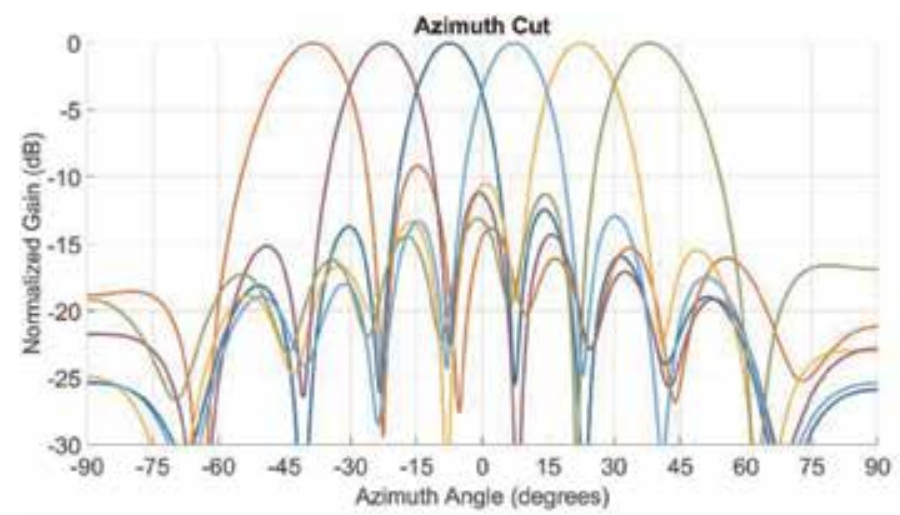

(a)

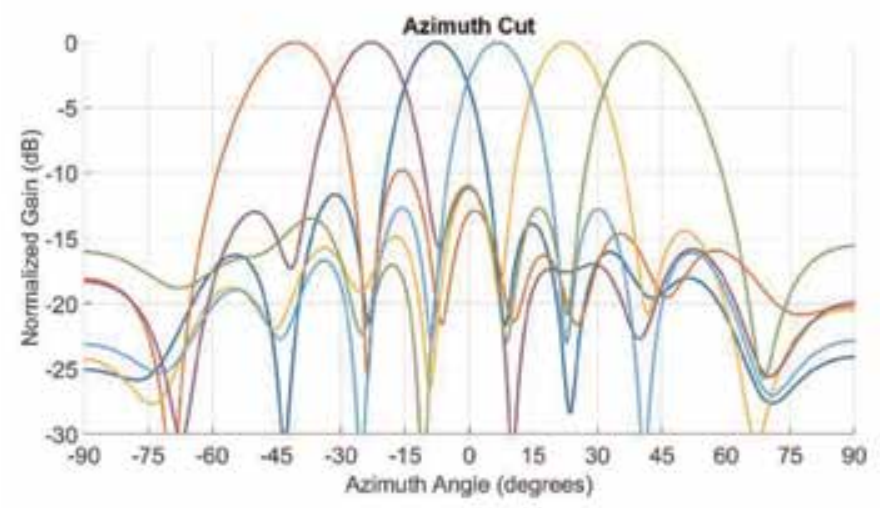

(b)

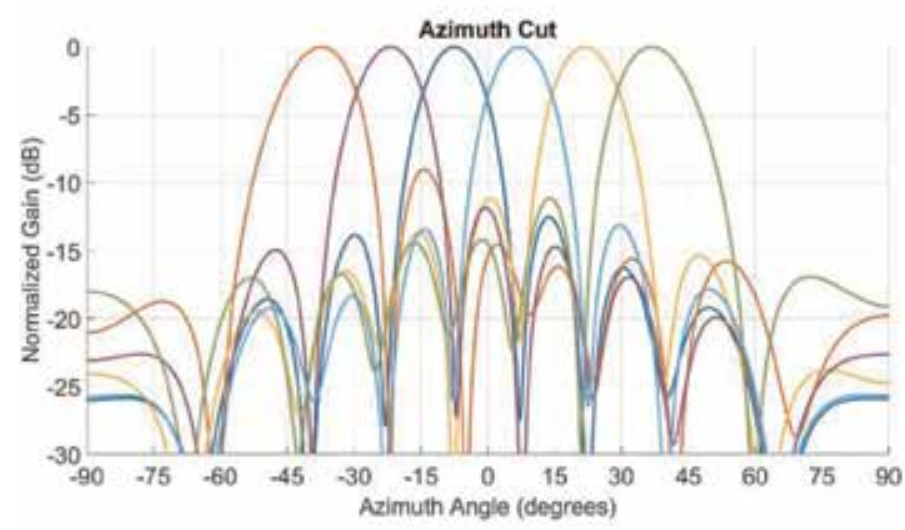

(c)

Figure 17.

Normalized radiation patterns for the mmWave multiple-beam array feed network under test (a) at $39.5 \mathrm{GHz}$ (b) at $38 \mathrm{GHz}($ c) at $40.5 \mathrm{GHz}$. 


\begin{tabular}{ccccccccc}
\hline \multirow{2}{*}{ Input RF (GHz) } & \multicolumn{7}{c}{ Output } \\
\cline { 2 - 9 } & B1 & B5 & B2 & B6 & B3 & B7 & B4 & B8 \\
\hline 39.0 & 10.4 & 9.5 & 9.1 & 8.3 & 7 & 6.1 & 5.6 & 4.8 \\
\hline 38.0 & 0 & 0 & 0.5 & 0.5 & 0 & 0 & 0.4 & 0.5 \\
\hline 40.5 & -13.2 & -12.7 & -11 & -9.8 & -9.2 & -8 & -6.5 & -5.2 \\
\hline
\end{tabular}

Table 7.

The phase error values for the center and two extreme frequencies of the RF generators.

phase distribution of the waveforms at the outputs of the OBM, previously obtained using the calculation in the VPI software.

The following outputs can be derived from our study:

- According to Table 6, the phase error values for the tested delay elements of PIC-based OBM are not more than $\pm 4^{\circ}$.

- According to Table 7, the phase error values for $8 \times 8$ PIC-based OBM under test are not more than $+10^{\circ} /-13^{\circ}$.

- The assessment showed that the approximate area of the PIC is near $270 \mathrm{~mm}^{2}$, which is approximately 50 times less than the size of the electronic counterpart [36].

- According to Figure 17, the replacement of phase shifters with TTD elements led to a change in the position of the main lobe maximum and an increase in the relative level of side lobes. However, in comparison with the ideal radiation patterns of Figures 9-11, the azimuth position change does not exceed $\pm 2^{\circ}$, and the increase in the level is not more than $2 \mathrm{~dB}$.

\section{Conclusion}

In the chapter, we explored and demonstrated the effectiveness of using reconfigurable multiple-beam array feed network based on millimeter-wave integrated photonics beamformers for the phased array antennas, which were known for a long time in the radar technique, in the small cells of the incoming fifthgeneration mobile communication systems. The study was carried out using a specific example of designing an $8 \times 8$ optical Butler matrix-based photonics-steered beamforming network of a transmitting phased array antenna for a pico-cell remote station operating in the $\mathrm{K}_{\mathrm{a}} / \mathrm{V}$-band with a $16 \%$ fractional bandwidth allocated as a promising one for future $5 \mathrm{G}$ systems. For this goal, we firstly reviewed the specialties of millimeter-wave photonics technique in $5 \mathrm{G}$ wireless networks of Radio-overFiber architecture. Then, to determine the input data for subsequent design, a theoretical background of array antenna beam steering using ideal models of phase shifters and true-time delay lines was presented. Comparison of the two most frequently used approaches to the design of multiple-beam antenna arrays based on Butler or Blass matrices showed the advantage of the first option for operation in the remote station of a $5 \mathrm{G}$ pico-cell.

A brief analysis of the available integrated millimeter-wave optical beamforming networks showed that the direction is at the initial stage of its development. A distinctive feature of the optical Butler matrix for designing beamformers is the 
Design of Reconfigurable Multiple-Beam Array Feed Network Based on Millimeter-Wave...

DOI: http://dx.doi.org/10.5772/intechopen.89076

simple possibility of reconfiguring the antenna system in two directions: frequency reconfiguration due to the rearrangement of the $\mathrm{RF}$ synthesizer and spatial reconfiguration due to the introduction of a multichannel optical switch at the input. As a result of the simulation experiments performed using VPIphotonics Design Suite and MATLAB software, for both the integrated optical Butler matrix itself and the beamformer based on it, an acceptable quality of beams formation in a particular 5G pico-cell was obtained.

\section{Acknowledgements}

This work was supported by the Russian Foundation for Basic Research, Grants No. 17-57-10002 and No. 18-29-20083.

\section{Conflict of interest}

The authors declare the lack of "conflict of interest."

\section{Author details}

Mikhail E. Belkin*, Dmitriy A. Fofanov, Tatiana N. Bakhvalova and Alexander S. Sigov

Scientific and Technological Center "Integrated Microwave Photonics", MIREA—Russian Technological University, Moscow, Russia

*Address all correspondence to: belkin@mirea.ru

\section{IntechOpen}

(C) 2019 The Author(s). Licensee IntechOpen. This chapter is distributed under the terms of the Creative Commons Attribution License (http://creativecommons.org/licenses/ by/3.0), which permits unrestricted use, distribution, and reproduction in any medium, provided the original work is properly cited. (cc) BY 


\section{References}

[1] Mailloux R. Phased Array Antenna Handbook. MA: Artech House, London; 2005. $496 \mathrm{p}$

[2] Andrews JG, Buzzi S, Choi W, Hanly SV, Lozano A, Soong ACK, et al. What will 5G Be? IEEE Journal on Selected Areas in Communications. 2014;32(6):1065-1082

[3] Waterhouse R, Novak D. Realizing 5G. IEEE Microwave Magazine. 2015;16(8):84-92

[4] Chen S, Zhao J. The requirements, challenges and technologies for $5 \mathrm{G}$ of terrestrial mobile telecommunication. IEEE Communications Magazine. 2014; 52(5):36-43

[5] Munn J. Our 5G future: In the fast lane with numerical simulation. Microwaves \& RF. Nov 16, 2016:48-50

[6] Frenzel L. Making 5G happen. Microwaves \& RF. 2017:1-5

[7] Browne J. What role will millimeter waves play in $5 \mathrm{G}$ wireless systems? Microwaves \& RF. 2018:38-42

[8] Novak D, Waterhouse R. Emerging disruptive wireless technologiesProspects and challenges for integration with optical networks. In: Optical Communication Conference and Exposition and the National Fiber Optic Engineers Conference (OFC/NFOEC); 17-21 March 2013. Anaheim, CA, USA: IEEE; 2013. pp. 1-3. DOI: 10.1364/ OFC.2013.OTu3E.2

[9] Novak D et al. Radio-over-fiber technologies for emerging wireless systems. IEEE Journal of Quantum Electronics. 2016;52(1):1-11

[10] Belkin ME, Fofanov D, Golovin V, Tyschuk Y, Sigov AS. Design and optimization of photonics-based beamforming networks for ultra-wide mmWave-band antenna arrays. In: Array Pattern Optimization. United Kingdom, London: IntechOpen; 2018. pp. 47-67. Available from: https://www. intechopen.com/online-first/designand-optimization-of-photonics-basedbeamforming-networks-for-ultra-widemmwave-band-antenna-a

[11] Boccardi F, Heath RW, Lozano A, Marzetta TL, Popovski P. Five disruptive technology directions for 5G. IEEE Communications Magazine. 2014; 52:74-80

[12] ITU. Final Acts of WRC-2015 World Radiocommunication Conference.

Resolution 238. Geneva; 2016. pp. 296-298

[13] Pi ZY, Khan F. An introduction to millimeter-wave mobile broadband systems. IEEE Communications Magazine. 2011;49(6):101-107

[14] Bakhvalova T, Belkin M, Fofanov D. Advances in fiber-wireless network architecture approach to the nextgeneration communication systems. In: Proceedings of the seventh International Conference on Advances in Computing, Communication and Information Technology-CCIT; 27-28

October 2018. Rome, Italy; 2018. pp. $62-67$

[15] Rohwer AB, Desrosiers DH, BachW, Estavillo H, Makridakis P, Hrusovsky R. Iridium main mission antennas- $\mathrm{A}$ phased array success story and mission update. In: 2010 IEEE International Symposium on Phased Array Systems and Technology; 12-15 October 2010. Waltham, MA, USA: IEEE; 2010. pp. 504-511. DOI:10.1109/ARRAY.2010. 5613319

[16] Frank J. Bandwidth criteria for phased-array antennas. In: Oliner A, 
Knittel G, editors. Phased-Array Antennas. Dedham, MA: Artech House; 1972. pp. 243-253

[17] Urick VJ, McKinney JD, Williams KJ. Fundamentals of Microwave Photonics. New Jersey: Hoboken; 2015. 489 p

[18] Matthews P. Practical photonic beamforming. In: International Topical Meeting on Microwave Photonics. MWP'99. Technical Digest (Cat. No. 99EX301); 17-19 November 1999. IEEE; 1999. pp. 271-274. DOI: $10.1109 /$ MWP.1999.819701

[19] Tur M. True time delay photonic beamforming: A review. In: IEEE International Conference on Microwaves, Communications, Antennas and Electronics Systems (COMCAS 2009). Tel Aviv; 2009

[20] Belkin ME, Golovin V, Tyschuk Y, Sigov AS. Comparison of RF photonicsbased beamformers for super-wide bandwidth phased array antennas. IOP Conference Series: Materials Science and Engineering. 2017;198:1-4

[21] Liu H, Liu X, Effenberger F, Chand N, Qi X, Li G. Optical implementation of Butler matrix for hardware-efficient multiuser beamforming. IEEE Photonics Journal. 2018;10(2):1-8

[22] Vidal B, Polo V, Corral JL, Martí J. Multibeam optical beamforming architectures for broadband wireless access networks. In: IST Mobile \& Wireless Telecommunications Summit 2003; 15-18 June; Aveiro (Portugal); 2003

[23] VPIphotonics Design SuiteTM. Available from: https://www. vpiphotonics.com/Tools/DesignSuite/ [Accessed: 18 September 2019]

[24] Fathpour S, Riza NA. Siliconphotonics-based wideband radar beamforming: Basic design. Optical Engineering. 2010;49(1):018201

[25] Zhuang L et al. Novel ring resonator-based integrated photonic beamformer for broadband phased array receive antennas-Part II: Experimental prototype. Journal of Lightwave Technology. 2010;28(1): 19-31

[26] Zhuang L et al. On-chip microwave photonic beamformer circuits operating with phase modulation and direct detection. Optics Express. 2014;22(14): 17079-17091

[27] Roeloffzen CGH, Oldenbeuving R, Timens RB, et al. Integrated optical beamformers. In: Optical Fiber Communication Conference 2015; Los Angeles, CA, USA, ThA2; 2015. pp. 1-3

[28] Alameh KE et al. Integrated microphotonic broadband smart antenna beamformer. In: Proceedings of the DELTA 2004. Second IEEE International Workshop on Electronic Design, Test and Applications; 28-30 January 2004; Perth, WA, Australia. IEEE; 2004. p. 208. DOI: 10.1109/ DELTA.2004.10028

[29] Roh W, Seol J-Y, Park J, et al. Millimeter-wave beamforming as an enabling technology for $5 \mathrm{G}$ cellular communications: Theoretical feasibility and prototype results. IEEE

Communications Magazine. 2014;52(2): 106-113

[30] Klein B, Jenning M, Hahnel R, Plettemeier D. Integrated Butler matrix and $1 \times 4$ antenna array for board-toboard communication in the mmWaverange. In: 2018 IEEE International Symposium on Radio-Frequency Integration Technology (RFIT); 15-17 August 2018; Melbourne, VIC, Australia. IEEE; 2018. DOI: 10.1109/ RFIT.2018.8524054

[31] Piqueras MA et al. Optically beamformed beam-switched adaptive 
antennas for fixed and mobile broadband wireless access networks. IEEE Transactions on Microwave Theory and Techniques. 2006;54(2): 887-899

[32] Liu Y et al. Integrated optical beamforming network for millimeter wave communications. In: 2017 International Topical Meeting on Microwave Photonics (MWP); 23-26 October 2017. Beijing, China: IEEE; 2017. pp. 1-4. DOI: $10.1109 /$ MWP.2017.8168704

[33] Roeloffzen C et al. Enhanced coverage though optical beamforming in fiber wireless networks. In: 2017 19th International Conference on Transparent Optical Networks (ICTON); 2-6 July 2017. Girona, Spain: IEEE; 2017. pp. 1-4. DOI: 10.1109/ ICTON.2017.8025129

[34] Grosskopf G et al. Photonic 60-GHz maximum directivity beam former for smart antennas in mobile broad-band communications. IEEE Photonics Technology Letters. 2002;14(8): 1169-1171. DOI: $10.1109 /$ LPT.2002.1022007

[35] Zhuang L, Marpaung D, Burla M, Beeker W, Leinse A, Roeloffzen C. Low-loss, high-index-contrast $\mathrm{Si}_{3} \mathrm{~N}_{4} / \mathrm{SiO}_{2}$ optical waveguides for optical delay lines in microwave photonics signal processing. Optics Express. 2011;19(23):23162-23170. DOI: 10.1364/OE.19.023162

[36] API Technologies. Available from: https://www.apitech.com/brands/ rf-solutions/weinschel-brand/ [Accessed: 18 September 2019] 


\title{
Characterization of Printed Podal Vivaldi Antenna $(8-18 \mathrm{GHz})$ on RT Duroid with Single and Double Cavity
}

\author{
P. Venu Madhav and M. Siva Ganga Prasad
}

\begin{abstract}
In today's modern communication systems, miniaturized and lightweight subsystems covering broad bandwidth are in much demand as they lead to realization of very compact and lightweight systems. A printed podal Vivaldi antenna with single as well as double cavities fed with strip line transmission line and operating from $\mathrm{X}$ band to $\mathrm{K}_{\mathrm{U}}$ band $(8-18 \mathrm{GHz})$ is proposed. The comparison of antenna performance for single cavity and double cavity is also reported. Using double cavity, the miniaturization of antenna is possible as compared to single cavity Vivaldi antenna. The antenna is first designed using conventional theoretical approaches. Later, it is simulated using a 3D EM simulation software, CST Microwave Studio ${ }^{\mathrm{TM}}$. The optimal value for taper length is $6.86455 \mathrm{~cm}$ and cavity diameter is $1.582 \mathrm{~cm}$. Finally, the design is physically fabricated using PCB technology for carrying out practical measurement. The antenna's input impedance characteristic is measured in the form of S-parameter and VSWR using Vector network analyzer. VSWR less than 3:1 is achieved over the band from 8 to $18 \mathrm{GHz}$. The radiation pattern measurements are carried out in anechoic chamber. The proposed Vivaldi antenna is used for digital data transmission via satellites and for voice/audio transmissions.
\end{abstract}

Keywords: Vivaldi, broadband antenna, microstrip, VSWR, characteristic impedance

\section{Introduction}

The recent explosion in information technology and wireless communications has created many opportunities for enhancing the performance of existing signal transmission and processing systems and has provided a strong motivation for developing novel antenna structure for systems that require wider bandwidths and higher data transmission.

In today's modern communication systems, miniaturized and lightweight subsystems covering broad bandwidth are in much demand as they lead to realization of very compact and lightweight systems. To accomplish this, small and lightweight antennas which cover wide bandwidth without much degradation in their performance are required to be designed and realized. As antenna 
dimensions are governed by wavelength of operating frequency, antenna miniaturization is a challenging and difficult task. Planar/printed antennas offer good solutions for the above class of problem. Vivaldi antennas are preferable in many applications due to high gain, simple structure and easy fabrication. They are mostly used in ultra-wideband and broadband applications. Printed antennas are being increasingly used as they are low profile and can be integrated on any printed circuit easily.

Most of the wireless communication systems suffer from co-channel interference and multipath effects. The co channel interference and multipath effects are addressed by using horn antennas that are placed in LOS, but these antennas are too bulky to be integrated with the rest of the wireless systems and suffer high cost of fabrication. For military and commercial applications wideband width antennas with high gain are preferred and Vivaldi antenna or planar tapered slot antenna (TSA) are better choice. As these antennas are support for multifunction communication applications because of their consistent impedance matching over a very broad operating frequency range, stable directional patterns, low profile and planar structures.

The Vivaldi and TSA's offer broadband operation, with low sidelobes but moderate gain.

Problem definition: The objective is to design the single cavity and double cavity Vivaldi antenna operating from 8 to $18 \mathrm{GHz}$ frequency to achieve VSWR less than 3:1 and comparison of antenna performance for single cavity and double cavity Vivaldi antenna.

\section{Vivaldi antenna}

A Vivaldi antenna gives significant advantages of efficiency, high gain, wide bandwidth and simple geometry. The Vivaldi antenna, having an exponentially tapered slot profile, is a type of tapered slot antenna (TSA). Lewis et al. [1] introduced tapered slot antenna as a broadband strip line array element capable of multi octave bandwidths in his study in 1974. Vivaldi antenna, is an exponentially tapered slot antenna, was originated by Gibson [2]. These antennas operate in the frequency range from below 2 to above $40 \mathrm{GHz}$ and offer significant gain and linear polarization.

Yngvesson et al. [3] compared three different TSAs, linearly tapered slot antenna (LTSA), constant width slot antenna (CWSA) and Gibson's exponentially tapered slot antenna, Vivaldi antenna.

Gazit [4] proposed two important changes to the traditional Vivaldi design. The use of a low dielectric substrate (cu clad, $\varepsilon=2.45$ ) instead of alumina and an antipodal slot line transition. This type of transition offers relatively wider bandwidth but, antipodal slot line transition has high cross polarization problem.

\section{Principle of operation}

The Vivaldi antenna belongs to travelling wave antennas. The principle of operation of the surface wave antennas can be divided into two sections: propagating section and radiating section.

\subsection{Vivaldi antenna design}

The design guidelines for a Vivaldi antenna are the following: 
Characterization of Printed Podal Vivaldi Antenna (8-18 GHz) on RT Duroid with Single... DOI: http://dx.doi.org/10.5772/intechopen.88727

1.the flare height and length should be greater or equal to half wavelength at minimum operating frequency;

2. the taper factor influences the impedance matching; and

3. the cavity diameter should be equal to $0.2 \lambda$ times the minimum operating frequency.

The separation between the conductors is smaller than one-half free space wavelength $\left(\lambda_{0} / 2\right)[5]$ and the waves travelling down the curved path along the antenna are tightly bound to the conductors in the propagating section. The energy gets radiated into the air in the radiating section where the slot width is increasing beyond the one-half wavelength. Radiation from high-dielectric substrates is very low and hence for antenna applications significantly low dielectric constant materials are chosen.

Apart from these, the design parameters are as follows:

1. antenna length;

2. antenna width;

3. mouth opening;

4. throat length;

5. edge offset; and

6. backwall offset.

Depending on the dimensions of the transmission line the characteristic impedance is calculated and the vivaldi antenna is printed on both sides of the substrate with a dielectric constant, $\varepsilon_{\mathrm{r}}=2.2$ and thickness of the substrate is $\mathrm{h}=0.508 \mathrm{~mm}$. The length and width of the antenna are optimized.

\subsection{Construction}

From Figure 1, the parameters of Vivaldi antenna are described as:

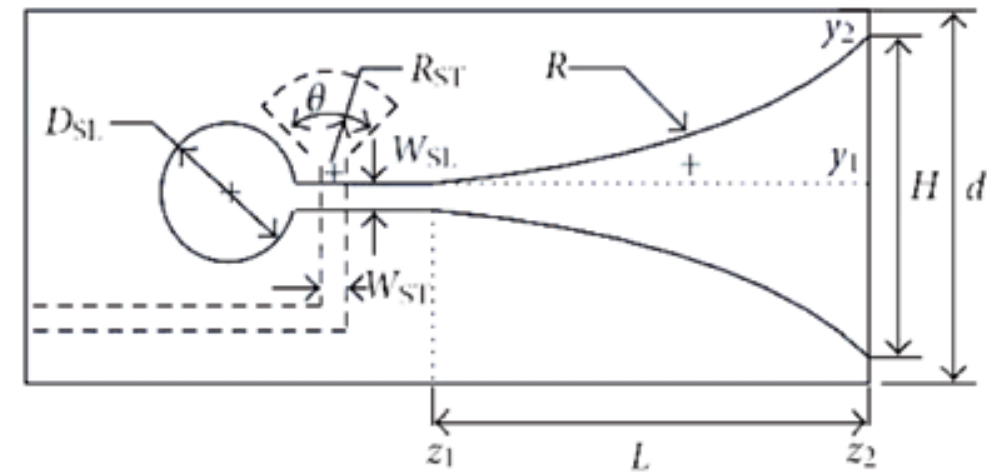

Figure 1.

Vivaldi antenna. 
- $\mathrm{D}_{\mathrm{SL}}$-diameter of slot line cavity;

- $\mathrm{W}_{\mathrm{SL}}$-width of slot line;

- $\mathrm{W}_{\mathrm{ST}}$-width of strip line;

- $\mathrm{R}_{\mathrm{ST}}$-radius of strip line stub;

- L-length of the taper;

- $\mathrm{H}$-height of the taper;

- R-exponential factor; and

- $\mathrm{d}$-height of the conductor.

The antenna consists of a tapered slot etched onto a thin film of metal. This can do either with or without a dielectric substrate on one side of the film. The tapered slot antennas work over a large frequency bandwidth and produce a symmetrical end-fire beam with appreciable gain and low side lobes. An important step in the design of the antenna is to find suitable feeding techniques for the Vivaldi.

The taper length should be on the order of one wavelength in the lowest working frequency. Besides, the taper length is also dependent on the cavity diameter and antenna length. An increase in the taper length improves the bandwidth.

The taper rate can be defined by an exponential.

$$
y= \pm A e^{R x}
$$

where $A=S w / 2,(R=) \frac{\ln \left(\frac{a}{S w}\right)}{\mathrm{La}}$ and $a$ is the antenna aperture at $L_{a}, S_{w}$ is the slot width at the antenna origin and $R$ is the taper rate.

\subsection{Bandwidth consideration}

To achieve a wider bandwidth, the following aspects need to be considered:

- the transition from the main input transmission line to the slot line is done for feeding the antenna;

- it is designed for a low reflection coefficient to match the potential of the antenna; and

- the dimensions and shape of the antenna, to obtain the required beam width, side lobes and back lobes, over the operating range of frequencies.

The length and width of the single cavity Vivaldi antenna are 38.5 and $15 \mathrm{~mm}$ respectively. Figure 2(a) and (b) shows the front and back view of simulated design of single cavity Vivaldi antenna which is excited using strip line [6] shown in Figure 2(c).

The double cavity Vivaldi antenna is compared with this single cavity Vivaldi antenna. The size of single cavity antenna is more than the double cavity antenna, 
Characterization of Printed Podal Vivaldi Antenna (8-18 GHz) on RT Duroid with Single... DOI: http://dx.doi.org/10.5772/intechopen.88727

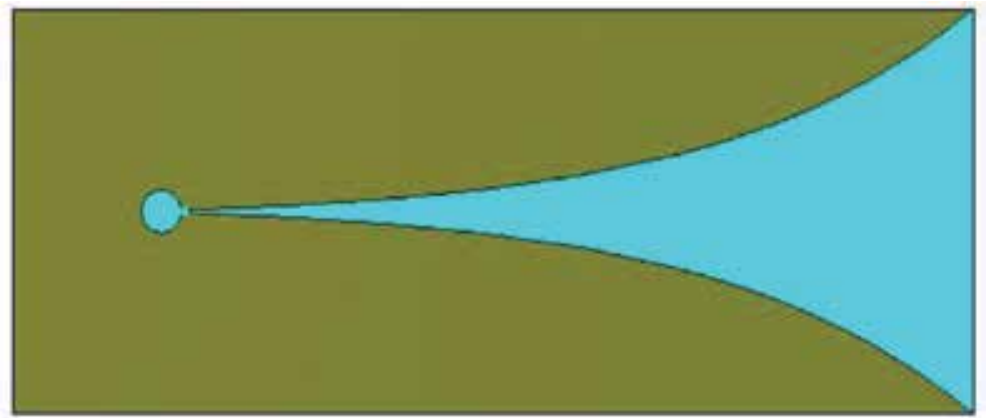

(a)

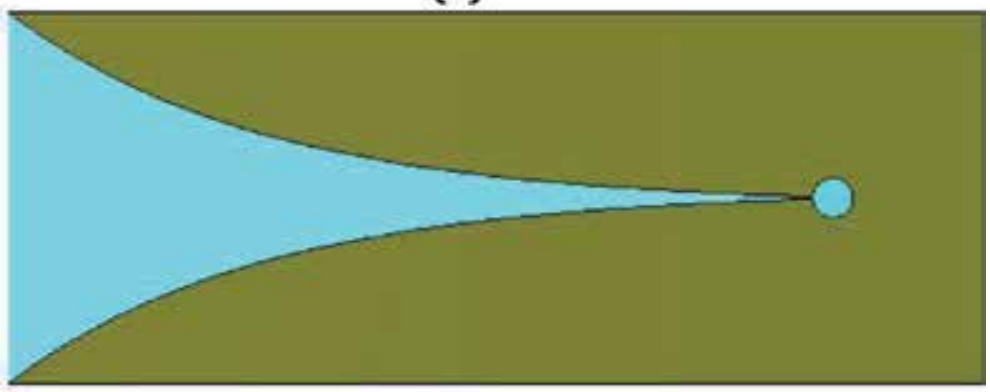

(b)

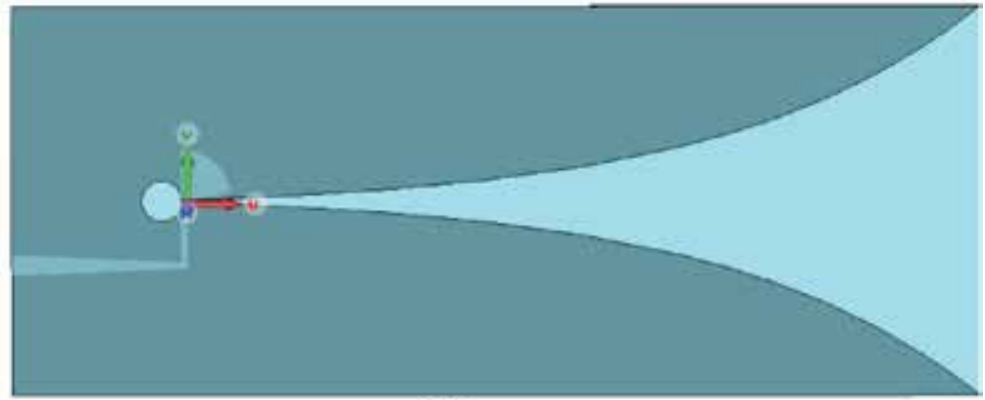

(c)

Figure 2.

(a) Front view of single cavity Vivaldi antenna; (b) back view of single cavity Vivaldi antenna; and (c) feeding arrangement of single cavity Vivaldi antenna.

the stub angles of tapered strip line feed also change. The length and width of double cavity Vivaldi antenna are 37.5 and $15 \mathrm{~mm}$ respectively. Figure 3(a)-(c) shows the front view, back view and feeding arrangement of double cavity Vivaldi antenna.

Parameter values for single cavity antenna is same as double cavity parameter list except some parameters are

stub start angle $=90^{\circ}$

stub angle $=80^{\circ}$

taper length $=6.86455$

cavity diameter $=1.582$

The VSWR-frequency plot for the Vivaldi antenna without SMA connector seems to have a high VSWR, which means the reflection of power is more and also the reflected wave amplitude is high from frequency 8 to $18 \mathrm{GHz}$. 


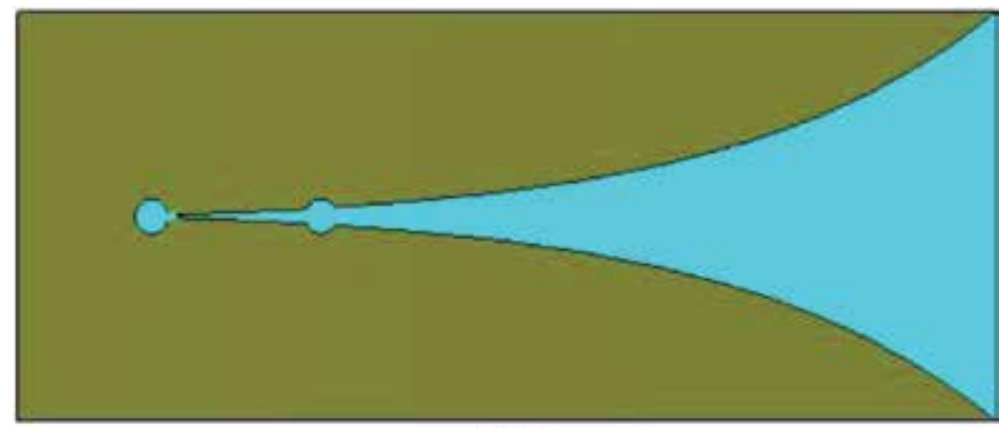

(a)

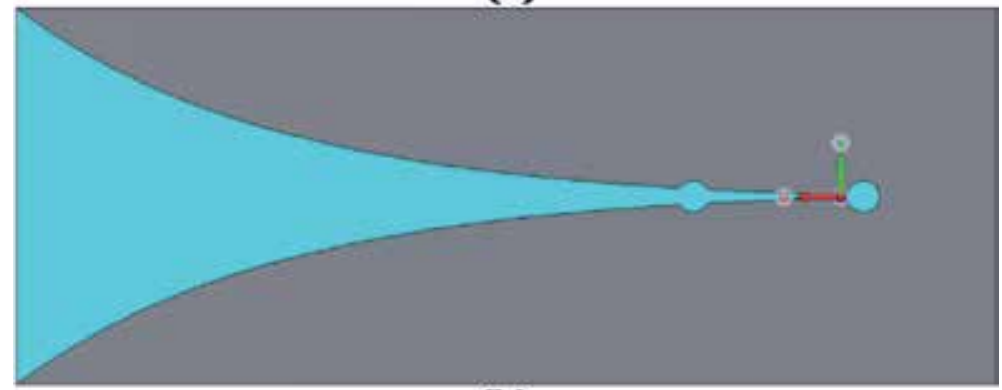

(b)

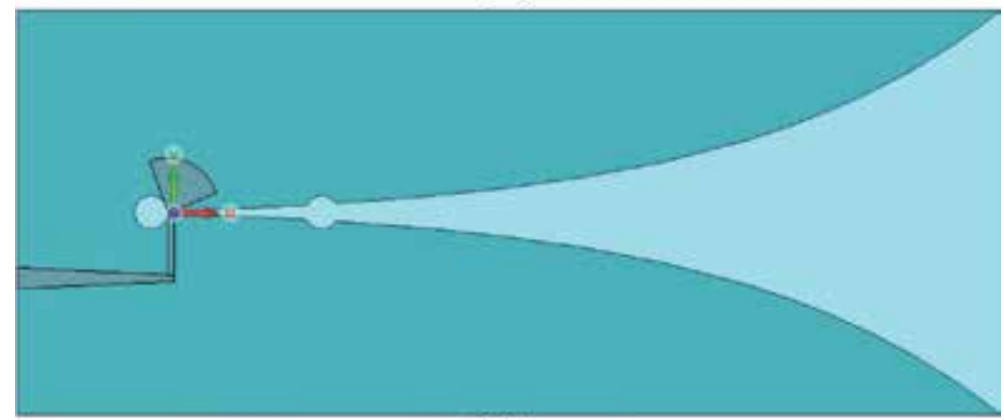

(c)

Figure 3.

(a) Front view of double cavity Vivaldi antenna; (b) back view of double cavity Vivaldi antenna; and (c) feeding arrangement of double cavity Vivaldi antenna.

To lower the VSWR, a connector is attached to the antenna for the required band of frequencies. Figure 4(a) and (b) shows the simulated single cavity Vivaldi antenna and double cavity Vivaldi antenna with SMA connector in CST software respectively. The designed SMA connector in CST software is shown in Figure 5.

\subsection{Fabricated Vivaldi antenna}

The single cavity and double cavity [7] Vivaldi antenna operating from 8 to $18 \mathrm{GHz}$ are fabricated individually on the substrate. The two substrates are joined to form a dual layered Vivaldi antenna [8] for both cavities separately. Normally, a FR4 substrate is used. This substrate is also the most commonly used PCB board which is cheap and easily fabricated.

Phase velocity of the propagating surface wave determines radiation performance [9]. Therefore, radiation pattern and performance is dependent upon 
Characterization of Printed Podal Vivaldi Antenna (8-18 GHz) on RT Duroid with Single... DOI: http://dx.doi.org/10.5772/intechopen.88727

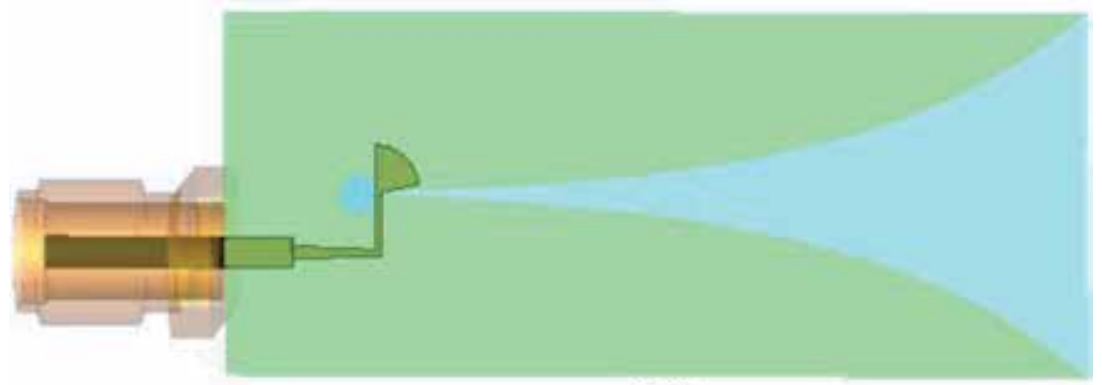

(a)

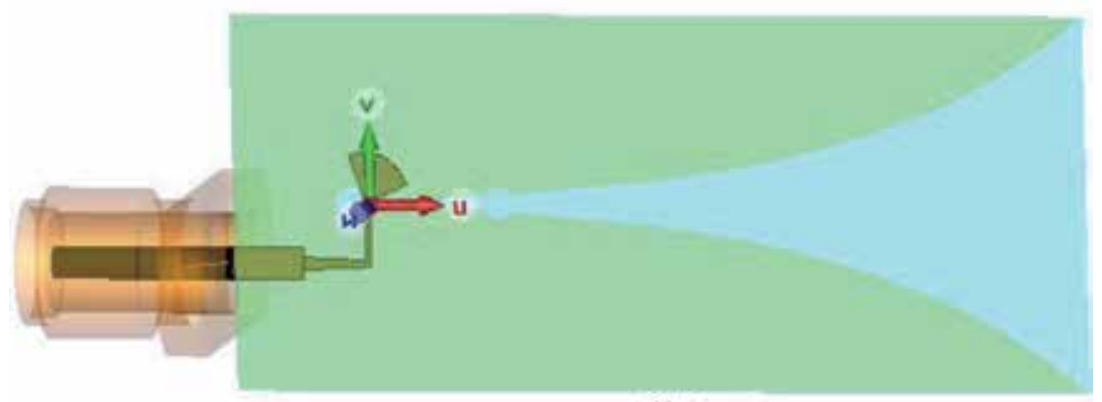

(b)

Figure 4.

(a) Single cavity Vivaldi antenna with SMA connector and (b) double cavity Vivaldi antenna with SMA connector.

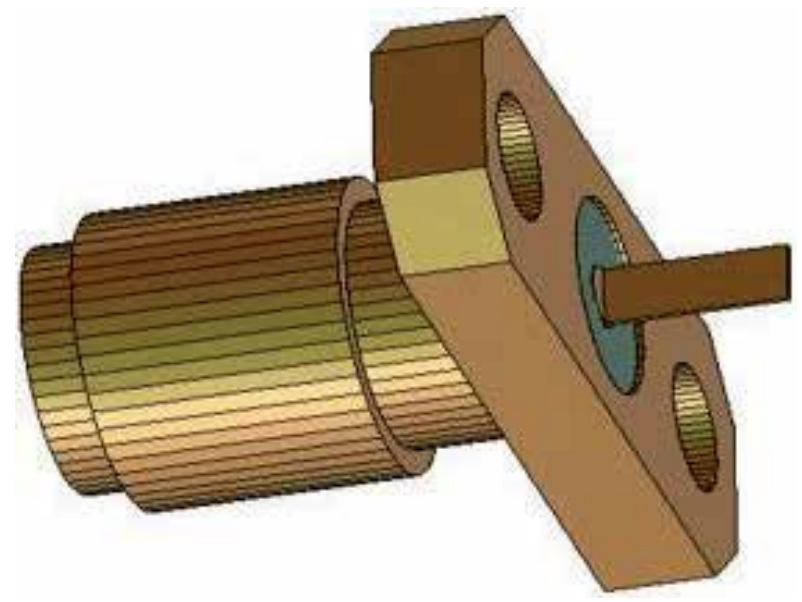

Figure 5.

Design of SMA connector in CST software.

substrate thickness and dielectric constant. The primary effect of the dielectric substrate is the narrowing of the main beam of the antenna. Low dielectric constant substrates maximize the antenna radiation by reducing the dielectric discontinuity at the end of the TSA.

Figure 6(a) and (b) shows the fabricated single cavity Vivaldi antenna and double cavity Vivaldi antenna respectively. These two antennas are tested individually. 


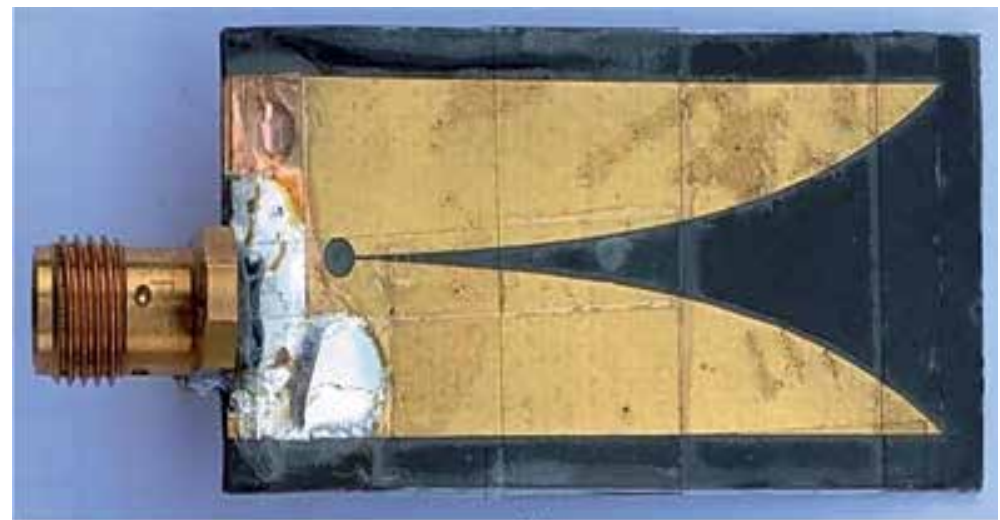

(a)

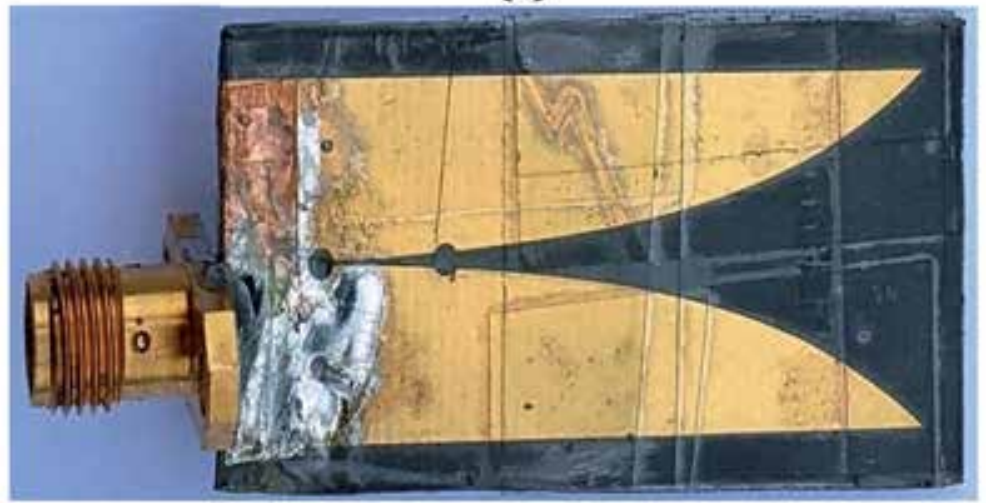

(b)

Figure 6.

(a) Fabricated single cavity Vivaldi antenna and (b) fabricated double cavity Vivaldi antenna.

\section{Results}

\subsection{VSWR}

The numerical simulation of directional antenna is done using CST software. The performance of the antenna does not improve monotonically as the parameters of the antenna changes. It must be optimized through analysis during simulation to get the optimum parameters.

The simulations for Vivaldi antenna operating in the band 8-18 GHz are done using CST. The obtained VSWR simulation results for the respective antenna are as shown in Figure 7(a) and (b).

As shown in Figure 7(a) and (b), the VSWR-frequency plot for the Vivaldi antenna without SMA connector seems to have a high VSWR, which means the reflection of power is more and the reflected wave amplitude is high from frequency 8 to $18 \mathrm{GHz}$.

To lower the VSWR, a connector is attached to the antenna for the required band of frequencies. Figure $\mathbf{8}(\mathbf{a})$ and (b) shows the VSWR with SMA connector in CST software for single cavity and double cavity respectively.

\subsection{Return loss}

The obtained return loss simulation results for the single cavity Vivaldi antenna and double cavity Vivaldi antenna are as shown in Figure 9(a) and (b), respectively. 
Characterization of Printed Podal Vivaldi Antenna (8-18 GHz) on RT Duroid with Single... DOI: http://dx.doi.org/10.5772/intechopen.88727

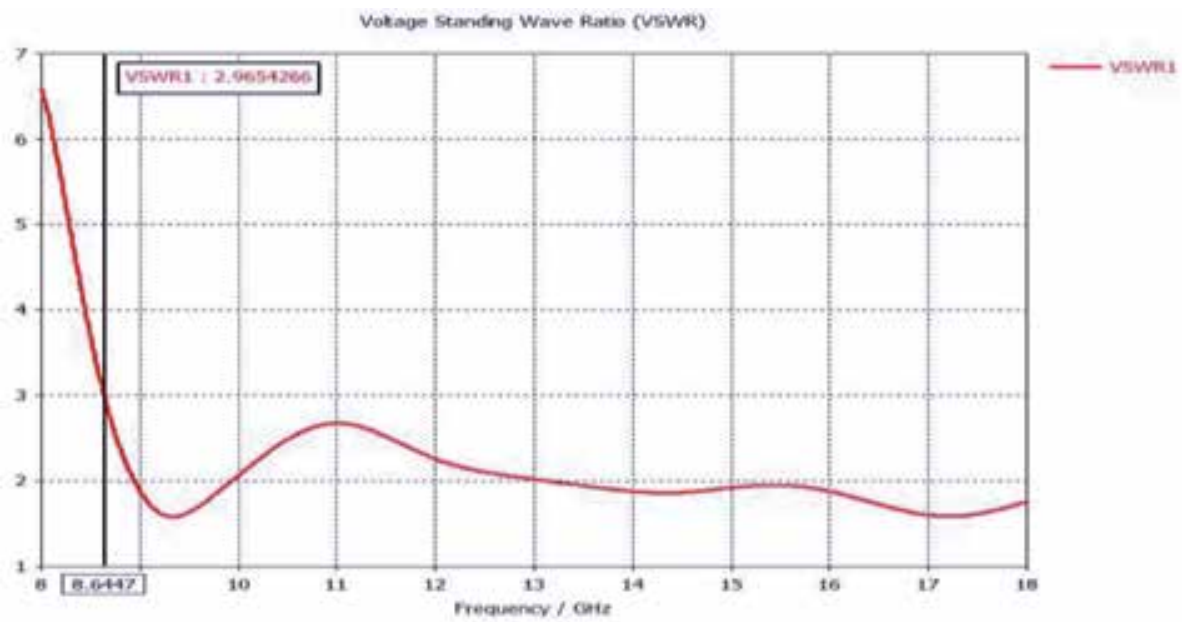

(a)

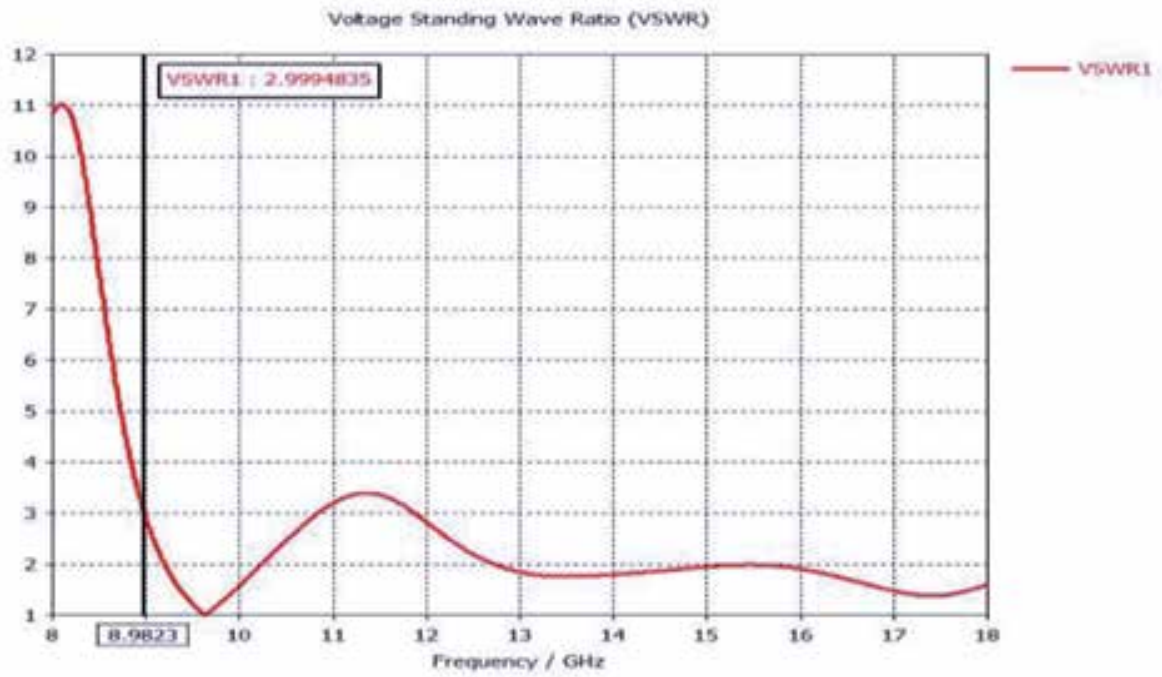

(b)

Figure 7 .

(a) Simulated VSWR for single cavity Vivaldi antenna without SMA connector and (b) simulated VSWR for double cavity Vivaldi antenna without SMA connector.

Usually the return loss is the loss of power in the signal that is returned/reflected by a discontinuity in a transmission line. A minor discontinuity can be observed with the terminating load or with a device inserted in the line.

Practically, VSWR for the desired band of frequencies is measured using a network analyzer. The VSWR of the proposed antenna was measured using Agilent ENA series Vector Network Analyzer (VNA) E5071C and was found to be less than 3:1 throughout the desired frequency band.

The measured VSWR for single cavity antenna and double cavity Vivaldi antenna are shown in Figure 10(a) and (b), respectively.

\subsection{Return loss measurement}

This discontinuity can be a mismatch with the terminating load or with a device inserted in the line. It is usually expressed as a ratio in decibels $(\mathrm{dB})$. 


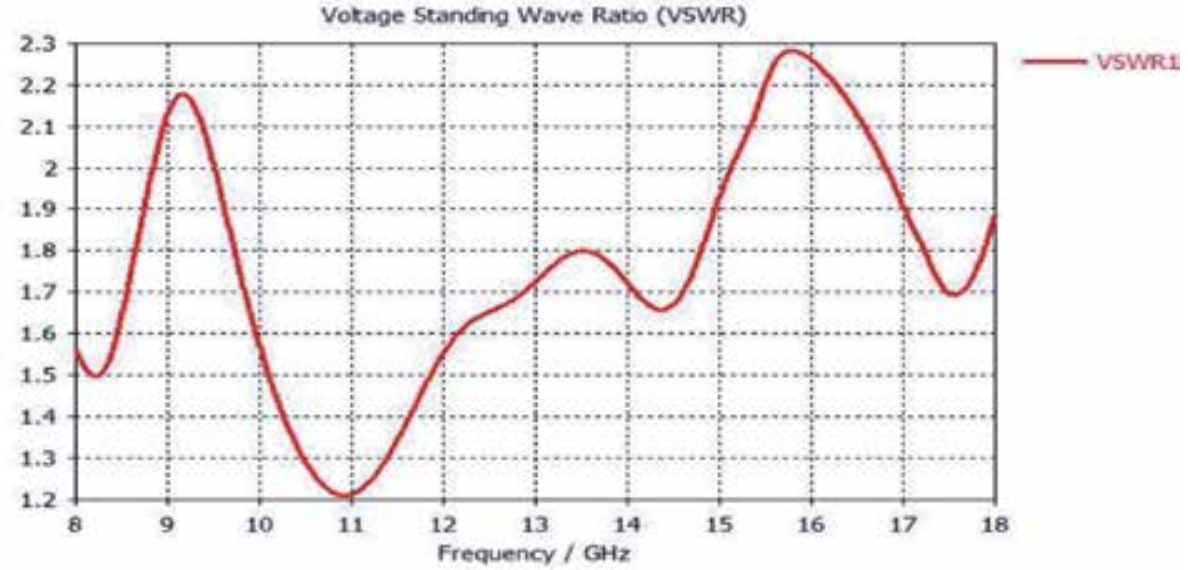

(a)

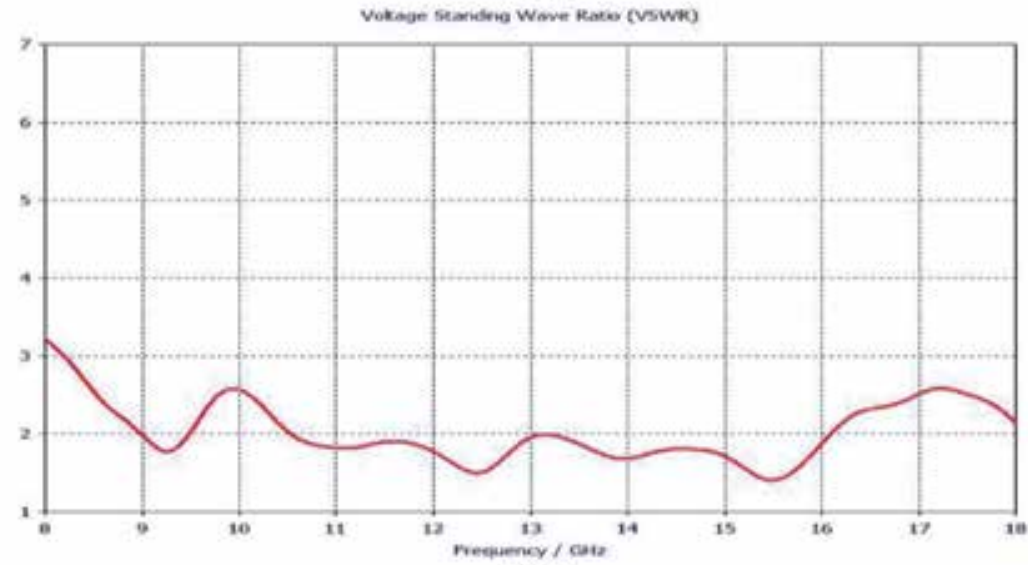

(b)

Figure 8.

(a) Simulated VSWR for single cavity Vivaldi antenna with SMA connector and (b) simulated VSWR for double cavity Vivaldi antenna with SMA connector.

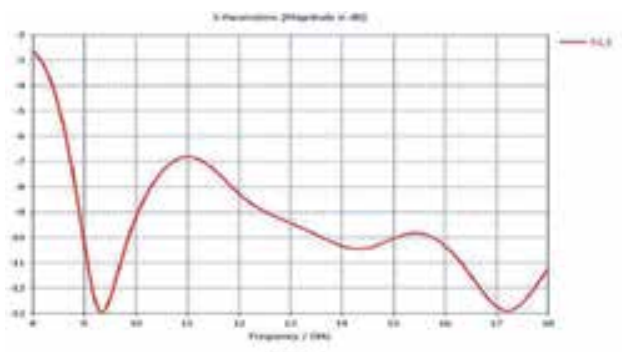

(a)

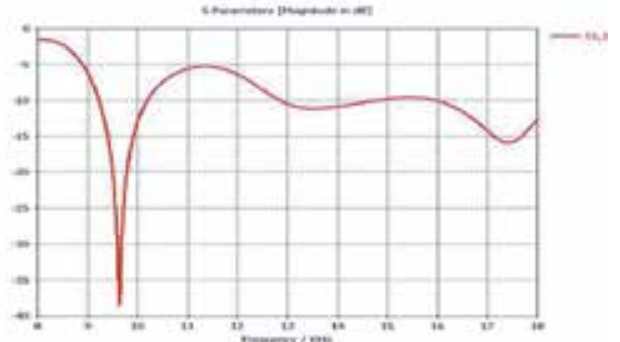

(b)

Figure 9.

(a) Simulated return loss for single cavity Vivaldi antenna and (b) simulated return loss for double cavity Vivaldi antenna.

The steps involved for measuring the return loss using network analyzer are given below:

1. select sweep frequency range by selecting start and stop frequency; 
Characterization of Printed Podal Vivaldi Antenna (8-18 GHz) on RT Duroid with Single... DOI: http://dx.doi.org/10.5772/intechopen.88727

2. select one port $\mathrm{S}_{11}$ for calibration measurement;

3. select log amplitude mode on display;

4. calibrate the network analyzer by connecting the standard short circuit, open circuit and matched loads at the test port. Observe the trace on the display to get a solid reference line; and

5. remove the standards and connect the antenna and observe the shift in the trace of the display. The display can be changed for obtaining the return loss, reflection coefficient, and impedance over the selected frequency band.

Return loss in $\mathrm{dB}=-20 \log (\rho)$, where $\rho$ is the reflection coefficient.

The measured return loss for single cavity Vivaldi antenna and double cavity Vivaldi antenna are shown in Figure 11(a) and (b), respectively.

\subsection{Anechoic chamber}

To provide a controlled environment, an all-weather capability, and security, and to minimize electromagnetic interference, indoor anechoic chambers have been developed as an alternative to outdoor testing. By this method, the testing is performed inside a chamber having walls that are covered with RF absorbers. The design of each is based on geometrical optics techniques, and each attempt to reduce or to minimize specular reflections. The phase difference between the

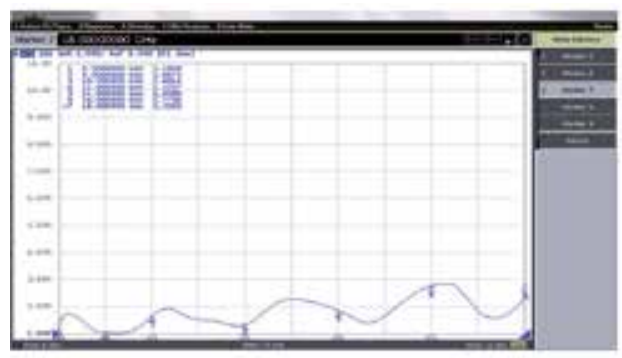

(a)

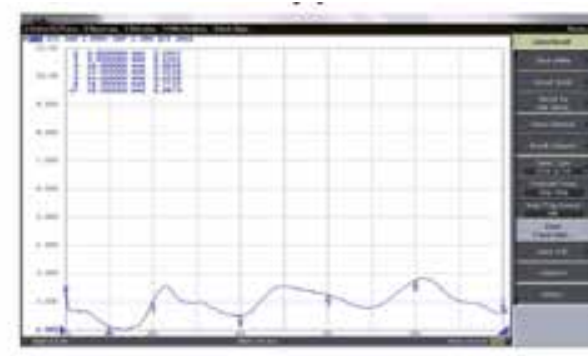

(b)

Figure 10.

(a) Measured VSWR for single cavity Vivaldi antenna and (b) measured VSWR for double cavity Vivaldi antenna.



(a)

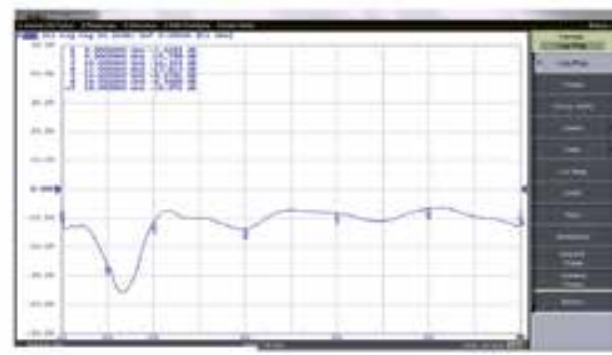

(b)

Figure 11.

(a) Measured return loss for single cavity Vivaldi antenna and (b) measured return loss for double cavity Vivaldi antenna. 


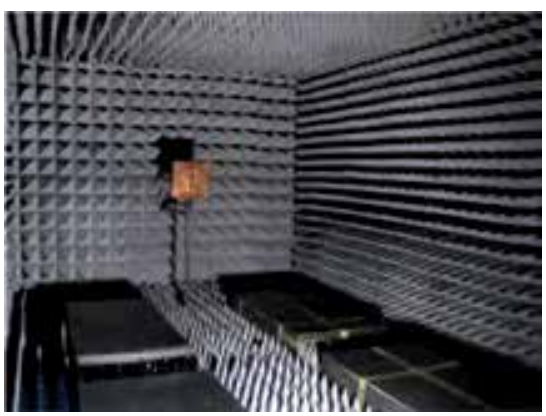

Figure 12.

Anechoic chamber.
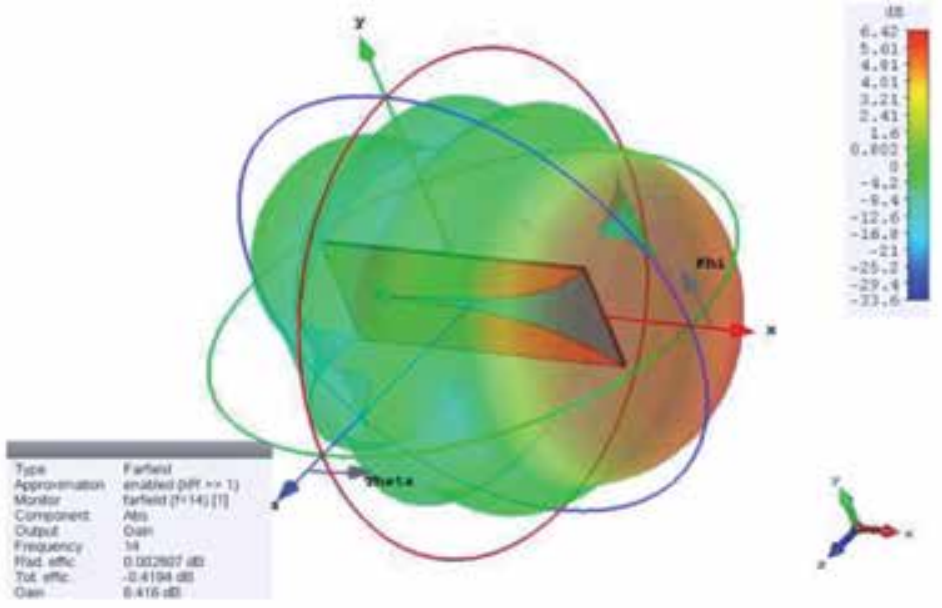

(a)

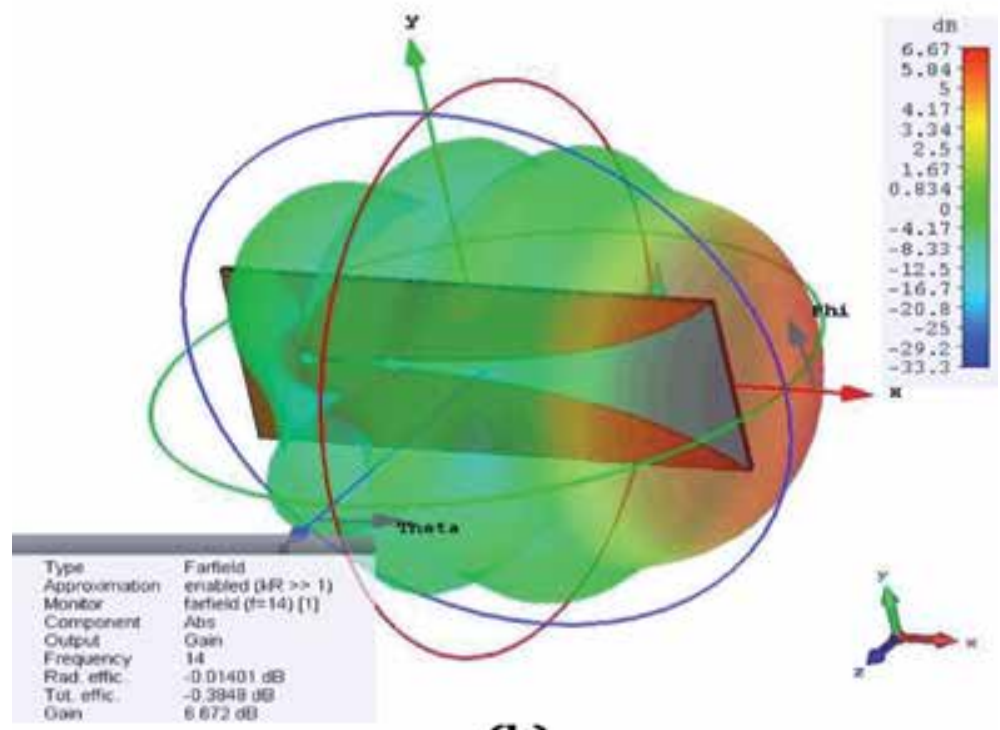

(b)

Figure 13.

(a) 3-D radiation pattern of single cavity Vivaldi antenna at $14 \mathrm{GHz}$ and (b) 3-D radiation pattern of double cavity Vivaldi antenna at $14 \mathrm{GHz}$. 
Characterization of Printed Podal Vivaldi Antenna (8-18 GHz) on RT Duroid with Single... DOI: http://dx.doi.org/10.5772/intechopen.88727

direct radiation and that reflected from the walls near the source can be made very small by properly locating the source antenna near the apex. Thus, the direct and reflected rays near the test antenna region add vectorially and provide a relatively smooth amplitude illumination taper. This can be illustrated by ray-tracing techniques (Figure 12).

\subsection{Radiation pattern}

The simulated 3D radiation patterns of printed Vivaldi antenna for single cavity and double cavity at frequency of $14 \mathrm{GHz}$ is shown in Figure 13(a) and (b) respectively.

\subsection{Plane patterns}

The E-plane and H-plane are the reference planes for linearly polarized waveguides, antennas and other microwave devices. The E-plane and H-plane patterns are also called as the polar plots or gain plots.

\subsubsection{E-plane}

The plane containing the $\mathrm{E}$ aperture and the direction of maximum radiation results in a linearly polarized antenna and it determines the orientation of the radio


(a)

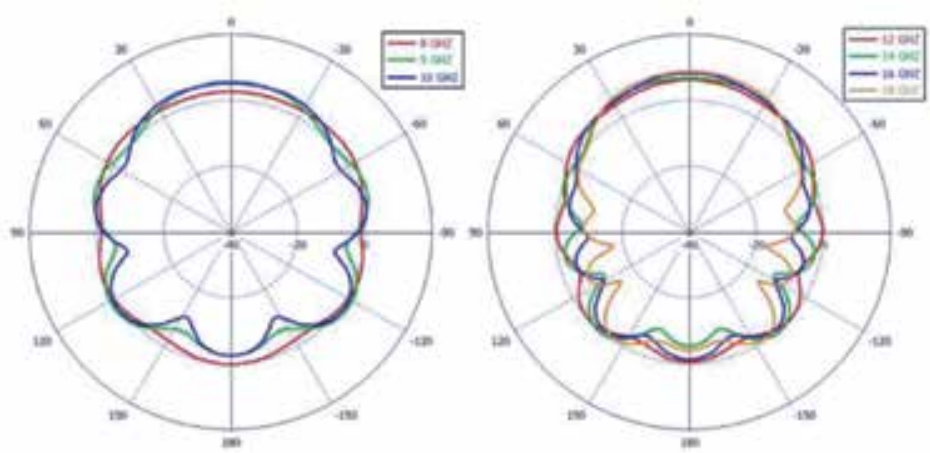

(b)

Figure 14.

(a) E plane patterns for single cavity Vivaldi antenna and (b) E plane patterns for double cavity Vivaldi antenna. 
wave. The E-plane (XY-plane at $\theta=90^{\circ}$ ) radiation patterns at different frequencies from 8 to $18 \mathrm{GHz}$ for single cavity and double cavity are as shown in Figure 14(a) and (b), respectively.

\subsubsection{H-plane}

The plane containing the magnetic field vector is referred as the $\mathrm{H}$ aperture and the direction of maximum radiation. The " $\mathrm{H}$ " plane lies at a right angle to the " $\mathrm{E}$ " plane. The H-plane (ZX-plane at $\phi=0^{\circ}$ ) radiation patterns at different frequencies from 8 to $18 \mathrm{GHz}$ for single cavity and double cavity are as shown in Figure 15(a) and (b), respectively.

\subsection{Gain of the antenna}

The method used for the gain measurement of the antenna is gain-transfer method. In this technique the standard gain antenna which is known is used to determine the test antenna. Initially relative gain measurements are performed, which when compared with the known gain of the standard antenna gives the

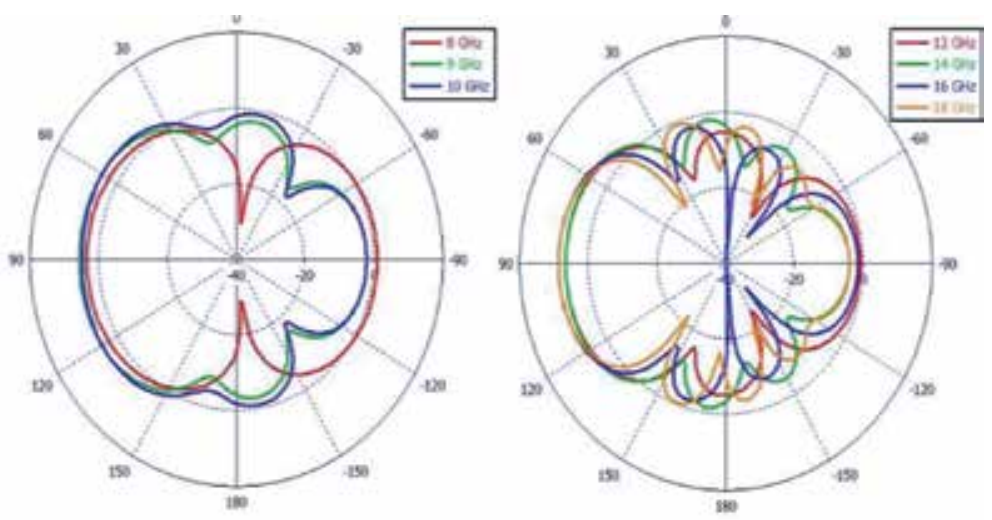

(a)
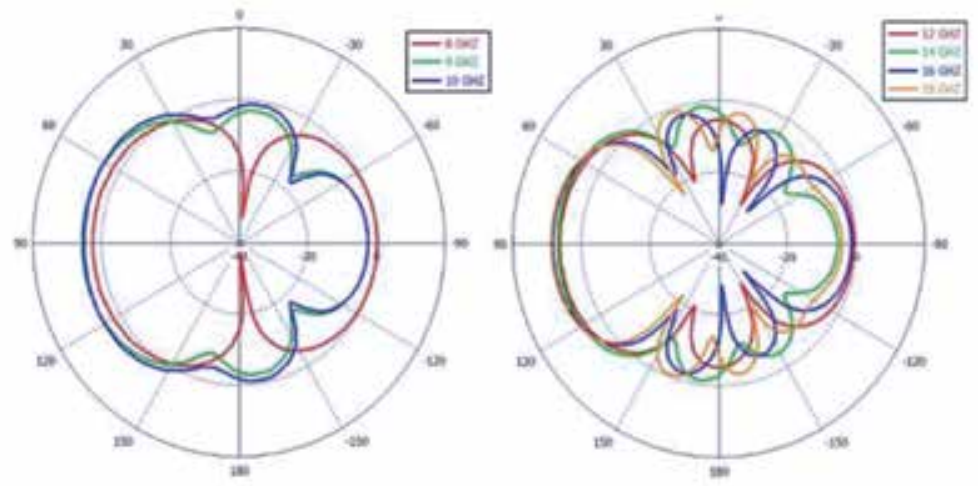

(b)

Figure 15.

(a) H plane patterns for single cavity Vivaldi antenna and (b) H plane patterns for double cavity Vivaldi antenna. 
Characterization of Printed Podal Vivaldi Antenna (8-18 GHz) on RT Duroid with Single... DOI: http://dx.doi.org/10.5772/intechopen.88727

absolute values. The simulated gain of single cavity Vivaldi antenna varies from 3.36 to $8.55 \mathrm{dBi}$ over the design frequency band as shown in Figure 16(a). The simulated gain of double cavity Vivaldi antenna varies from 2.62 to $8.65 \mathrm{dBi}$ over the design frequency band as shown in Figure 16(b).

For measuring the gain of the antenna under test (AUT), formula is given by

Gain of AUT $(\mathrm{dB})=$ antenna power $(\mathrm{dB})$ - reference antenna power $(\mathrm{dB})$

+ gain (reference antenna)

The measured gain plots of single cavity Vivaldi antenna and double cavity Vivaldi antenna are shown in Figure 17(a) and (b), respectively.

Finally, single cavity Vivaldi antenna gives the efficient impedance bandwidth than the double cavity Vivaldi antenna.

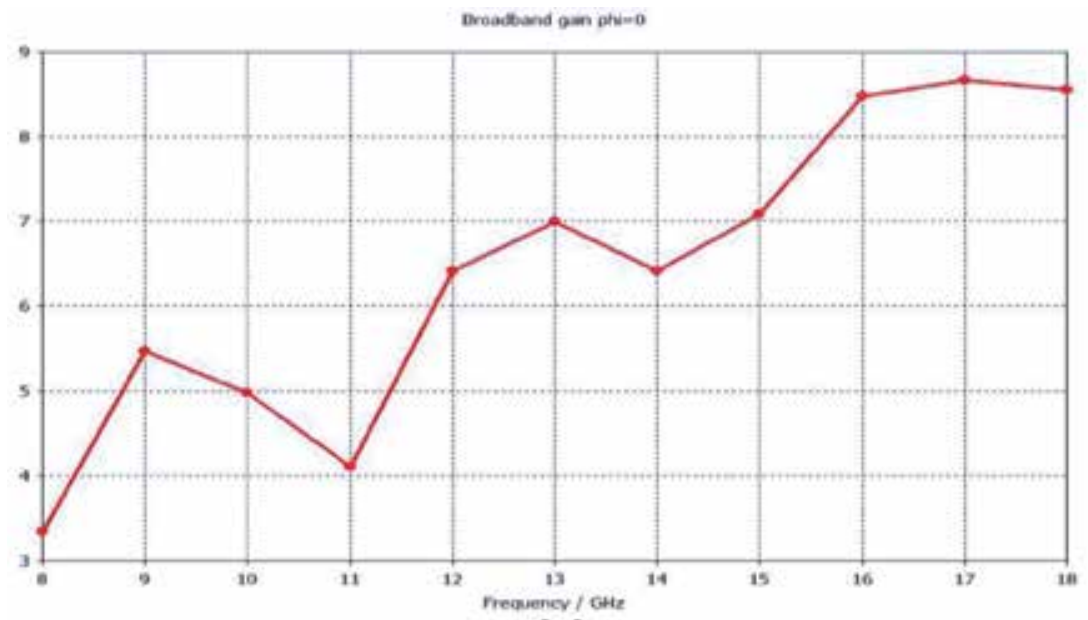

(a)

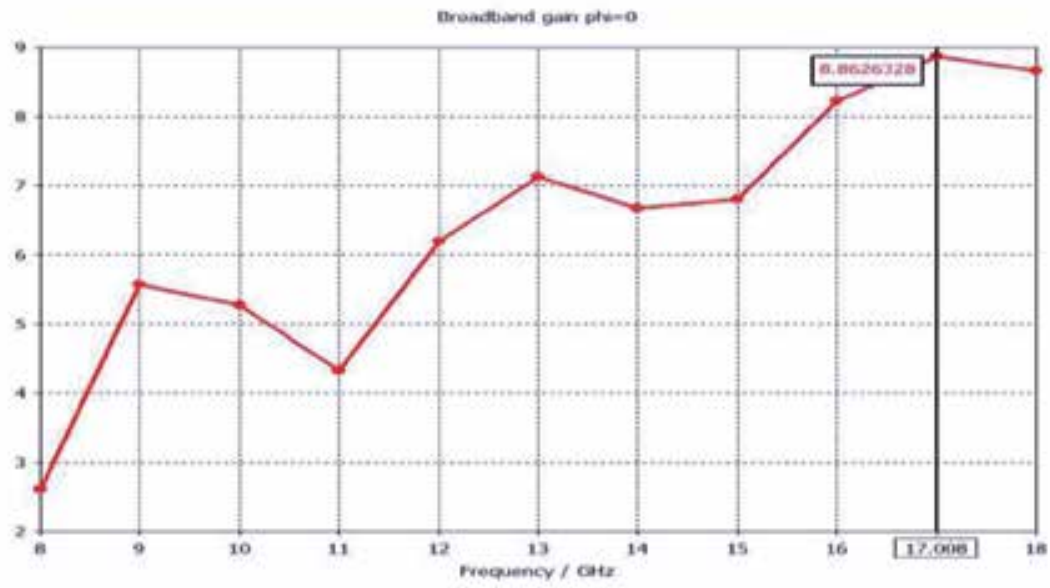

(b)

Figure 16.

(a) Simulated gain plot of single cavity Vivaldi antenna and (b) simulated gain plot of double cavity Vivaldi antenna. 


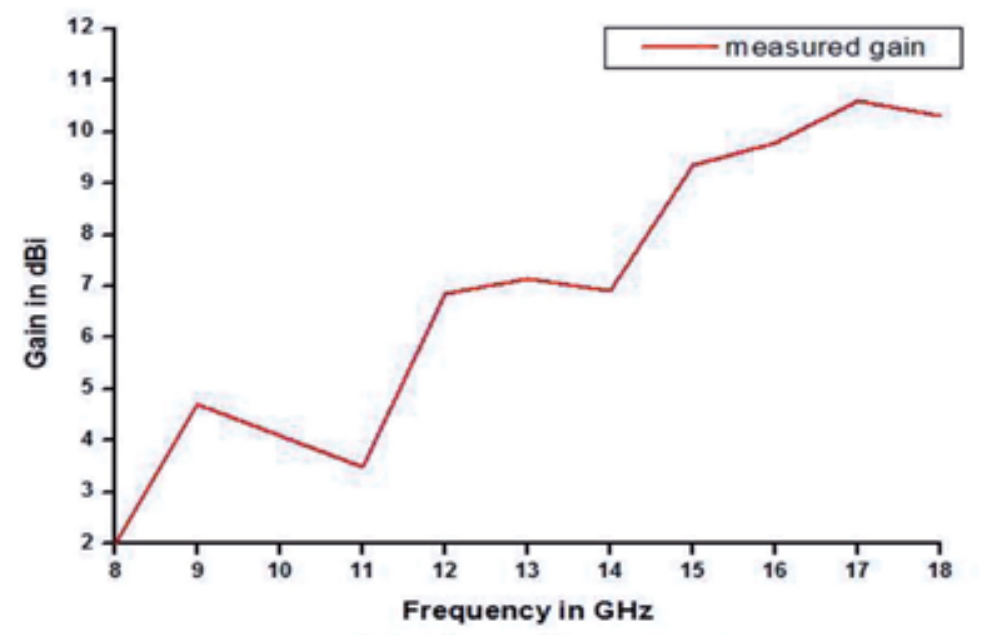

(a)

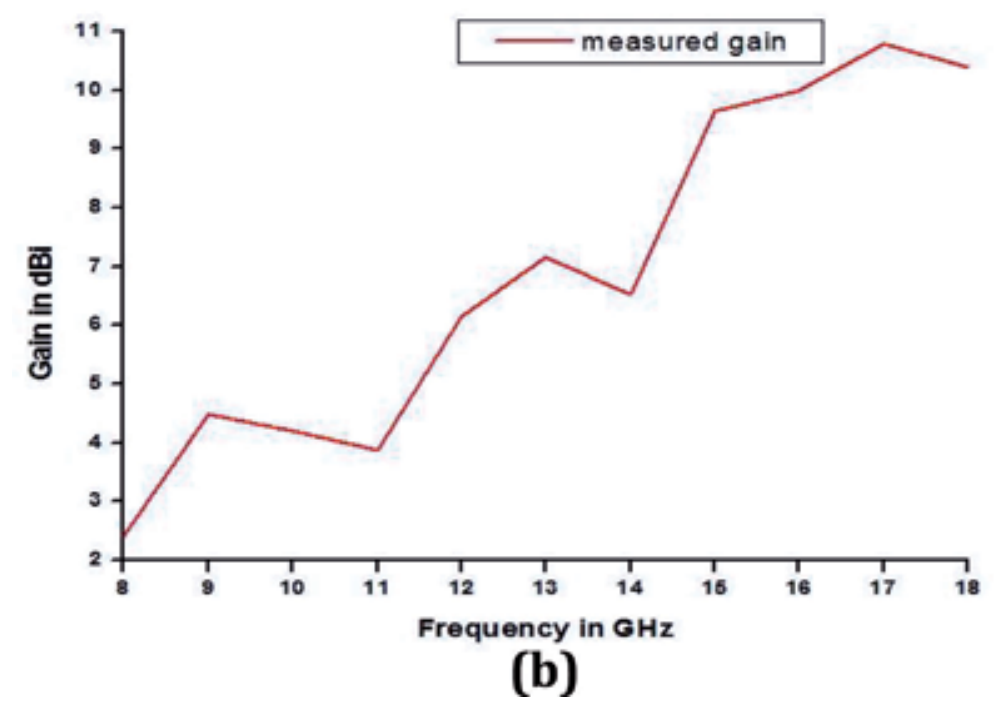

Figure 17.

(a) Measured gain plot of single cavity Vivaldi antenna and (b) measured gain plot of double cavity Vivaldi antenna.

\section{Conclusion}

The comparison of antenna performance for single cavity Vivaldi antenna and double cavity Vivaldi antenna are reported. The single cavity and double cavity have same performances, but single cavity gives efficient impedance bandwidth than the double cavity. The gain of double cavity is better than single cavity at higher frequencies and antenna miniaturization also possible.

The simulated and measured results of the proposed antenna were compared and found to be in good agreement. The optimum performance of the antenna in simulation is obtained by using parametric analysis. VSWR less than 3:1 is achieved over the band from 8 to $18 \mathrm{GHz}$. The antenna due to its compactness and lightweight serves its applications in radio communications, avionics, spectrum monitoring and military system. It also finds applications in digital data transmission via satellites and for voice/audio transmissions. 
Characterization of Printed Podal Vivaldi Antenna (8-18 GHz) on RT Duroid with Single... DOI: $h$ ttp://dx.doi.org/10.5772/intechopen.88727

\section{Acknowledgements}

At the outset, I would like to take this as an opportunity to convey my gratitude to IntechOpen publishing house \& their support team's for their consistent guidance, undue financial support at every step in bringing out this chapter in their book has left me in awe. The process that followed in developing this chapter for the book has been a very stimulating experience. Couple of months of intensive deliberations, several stages of drafting and redrafting of the manuscript to meet the standards of the book and the publishing house has enriched my writing skills. I would like to thank my parents \& family members for their love and guidance, and the management of my college, PVP.Siddhartha Institute of Technology, for constant encouragement.

\section{Author details}

P. Venu Madhav ${ }^{1,2 *}$ and M. Siva Ganga Prasad ${ }^{2}$

1 Department of Electronics and Communication Engineering, PVPSIT,

Vijayawada, India

2 Department of Electronics and Communication Engineering, KL University, Guntur, India

*Address all correspondence to: venu7485@gmail.com

\section{IntechOpen}

(C) 2019 The Author(s). Licensee IntechOpen. This chapter is distributed under the terms of the Creative Commons Attribution License (http://creativecommons.org/licenses/ by/3.0), which permits unrestricted use, distribution, and reproduction in any medium, provided the original work is properly cited. (cc) BY 


\section{References}

[1] Lewis LR, Fasset M, Hunt J. A broadband stripline array. In: IEEE A P-S Synip. Antennas \& Propagation Society; June 1974. p. 335

[2] Gibson PJ. The vivaldi aerial. In: Proceedings of the 9th European Microwave Conference. Brighton, UK, Sevenoaks: Microwave Exhibitions and Publishers; Oct. 1979. pp. 101-105

[3] Yngvesson KS, Korzienowski TL, Kim YS, Kollberg EL, Johansson JF. Endfire tapered slot antenna on dielectric substrates. IEEE Transactions on Antennas and Propagation. December 1985;AP-33(12):1392-1400

[4] Gazit E. Improved design of a vivaldi antenna. In: IEE Proceedings H. Microwaves, Antennas and Propagation; April 1988

[5] Langley JDS, Hall PS, Newham P. Novel ultrawide-bandwidth vivaldi antenna with low cross polarization. Electronic Letters. November 1993;29(23):2004-2005

[6] Langley JDS, Hall PS, Newham P. Balanced antipodal vivaldi antenna for wide bandwidth phased arrays. IEEE Proceedings of the Antennas and Propagation. April 1996;143(2):97-102

[7] Sloan R, Zinieris MM, Davis LE. A broadband microstrip to slot line transition. Microwave and Optical Technology Letters. August 1998;18(5):339-342

[8] Schaubert DH, Shin J. A parameter study of stripline-fed vivaldi notch antenna arrays. IEEE Transactions on Antennas and Propagation. May 1999;47(5):879-886

[9] Colburn JS, Rahmat-Samii Y. Linear taper slot antenna directivity improvement via substrate perforation:
A FDTD evaluation. In: IEEE Antennas and Propagation Society International Symposium. IEEE Antennas and Propagation Society International Symposium; Vol. 2. 21-26 Jun 1998. pp. 1176-1179 



\section{Edited by Ertugrul Aksoy}

The need to develop technology and communication necessitates the design of flexible and high-capacity radiating systems in today's communication infrastructure. In this context, antenna arrays are the ideal solution and have been one of the priority research subjects of the science community dealing with electromagnetics from past to present. Optimization of an array may be performed in various ways such as the optimization of excitation, reflector structure, feed network, etc. depending on the array structure. This book is a collection of seven research studies focused on the optimization of array structures in classical phased array or time modulation, including radiator, reflector, feed network, and radiating element optimizations. 